\title{
WestVirginiaUniversity
}

THE RESEARCH REPOSITORY @ WVU

Graduate Theses, Dissertations, and Problem Reports

2020

\section{Searching for Needles in the Cosmic Haystack}

Thomas Ryan Devine

West Virginia University, tdevine4@mail.wvu.edu

Follow this and additional works at: https://researchrepository.wvu.edu/etd

Part of the Artificial Intelligence and Robotics Commons, Other Astrophysics and Astronomy

Commons, and the Theory and Algorithms Commons

\section{Recommended Citation}

Devine, Thomas Ryan, "Searching for Needles in the Cosmic Haystack" (2020). Graduate Theses,

Dissertations, and Problem Reports. 7727.

https://researchrepository.wvu.edu/etd/7727

This Dissertation is protected by copyright and/or related rights. It has been brought to you by the The Research Repository @ WVU with permission from the rights-holder(s). You are free to use this Dissertation in any way that is permitted by the copyright and related rights legislation that applies to your use. For other uses you must obtain permission from the rights-holder(s) directly, unless additional rights are indicated by a Creative Commons license in the record and/ or on the work itself. This Dissertation has been accepted for inclusion in WVU Graduate Theses, Dissertations, and Problem Reports collection by an authorized administrator of The Research Repository @ WVU.

For more information, please contact researchrepository@mail.wvu.edu. 
Graduate Theses, Dissertations, and Problem Reports

2020

Searching for Needles in the Cosmic Haystack

Thomas Ryan Devine

Follow this and additional works at: https://researchrepository.wvu.edu/etd

Part of the Artificial Intelligence and Robotics Commons, Other Astrophysics and Astronomy Commons, and the Theory and Algorithms Commons 


\title{
Searching for Needles in the Cosmic Haystack
}

\author{
Thomas Ryan Devine \\ Dissertation submitted to the \\ Benjamin M. Statler College of Engineering and Mineral Resources \\ at West Virginia University \\ in partial fulfillment of the requirements \\ for the degree of \\ Doctor of Philosophy \\ in \\ Computer Science \\ Katerina Goseva-Popstojanova, Ph.D., Chair \\ Kevin Bandura, Ph.D. \\ Elaine Eschen, Ph.D. \\ Duncan Lorimer, Ph.D. \\ Maura McLaughlin, Ph.D. \\ Natalia Schmid, Ph.D. \\ Lane Department of Computer Science and Electrical Engineering \\ Morgantown, West Virginia \\ 2020
}

Keywords: machine learning, classification, big data, pulsar, radio astronomy

Copyright 2020 Thomas Ryan Devine 


\author{
Abstract \\ Searching for Needles in the Cosmic Haystack \\ by \\ Thomas Ryan Devine \\ Doctor of Philosophy in Computer Science \\ West Virginia University \\ Katerina Goseva-Popstojanova, Ph.D., Chair
}

Searching for pulsar signals in radio astronomy data sets is a difficult task. The data sets are extremely large, approaching the petabyte scale, and are growing larger as instruments become more advanced. Big Data brings with it big challenges. Processing the data to identify candidate pulsar signals is computationally expensive and must utilize parallelism to be scalable. Labeling benchmarks for supervised classification is costly. To compound the problem, pulsar signals are very rare, e.g., only $0.05 \%$ of the instances in one data set represent pulsars. Furthermore, there are many different approaches to candidate classification with no consensus on a best practice. This dissertation is focused on identifying and classifying radio pulsar candidates from single pulse searches. First, to identify and classify Dispersed Pulse Groups (DPGs), we developed a supervised machine learning approach that consists of RAPID (a novel peak identification algorithm), feature extraction, and supervised machine learning classification. We tested six algorithms for classification with four imbalance treatments. Results showed that classifiers with imbalance treatments had higher recall values. Overall, classifiers using multiclass RandomForests combined with Synthetic Majority Oversampling TEchnique (SMOTE) were the most efficient; they identified additional known pulsars not in the benchmark, with less false positives than other classifiers. Second, we developed a parallel single pulse identification method, D-RAPID, and introduced a novel automated multiclass labeling (ALM) technique that we combined with feature selection to improve execution performance. D-RAPID improved execution performance over RAPID by a factor of 5. We also showed that the combination of ALM and feature selection sped up the execution performance of RandomForest by $54 \%$ on average with less than a $2 \%$ average reduction in classification performance. Finally, we proposed CoDRIFt, a novel classification algorithm that is distributed for scalability and employs semi-supervised learning to leverage unlabeled data to inform classification. We evaluated and compared CoDRIFt to eleven other classifiers. The results showed that CoDRIFt excelled at classifying candidates in imbalanced benchmarks with a majority of non-pulsar signals (>95\%). Furthermore, CoDRIFt models created with very limited sets of labeled data (as few as 22 labeled minority class instances) were able to achieve high recall (mean $=0.98)$. In comparison to the other algorithms trained on similar sets, CoDRIFt outperformed them all, with recall $2.9 \%$ higher than the next best classifier and a 35\% average improvement over all eleven classifiers. CoDRIFt is customizable for other problem domains with very large, imbalanced data sets, such as fraud detection and cyber attack detection. 


\section{Acknowledgments}

This work was supported by NSF award \#1458952 and by the WVU Foundation's Ruby Distinguished Doctoral Fellowship. I would like to thank the NSF and WVU Foundation for their support.

First, I would like to acknowledge and thank my committee chair and advisor, Dr. Katerina Goseva-Popstojanova, for giving a local boy from a small university the opportunity to work with her and her students. This dissertation would not be possible without her guidance and support. Her many long nights of editing and assistance with navigating the nuances of Academia are greatly appreciated, more than words on a page can express.

I am also deeply indebted to many members of the astronomical community for their willingness to allow a computer scientist into their midst, starting with Dr. Arun Venkataraman at the Arecibo Observatory. I owe many thanks to Drs. Maura McLaughlin, Duncan Lorimer, and Jayanth Chennamangalam for sharing their wealth of knowledge about the nature of pulsars and radio data with me. I would also like to thank Weiwei Zhu and the PALFA collaboration for making these data available and for helpful discussions along the way.

I owe a debt of gratitude to my committee members from the WVU Statler College, several of whom picked up this gig on short notice without a complaint. That kind of dedication to students is what makes WVU a special place, and I am blessed to be among you. Many thanks are necessary to Dr. Jim Harner, for teaching me about Spark and getting me interested in high performance computing. I also owe my thanks to Chris Grant, for listening to my ideas and opening my mind to many, many others.

I couldn't have made any of this possible without the inspiration and guidance I received from several of my undergraduate professors and then colleagues at Fairmont State University. From becoming a CS major at the suggestion of Professor Don Tobin, to learning how to excel at solving problems and developing software under the excellent tutelage of Profes- 
sors Randy Baker and Ted Larue, to being inspired to reach for the stars by Dr. Mahmood Hossain, the quality of CS education I received at FSU set me up for success in life and I am forever grateful.

I would like to thank my friends, housemates, and family for always believing in me and propping me up when I felt like falling down. I would not be who I am without the love of my cantankerous Grandma Anne, who gave me the gifts of poetry, curiosity, and imagination. My mother Mary Ann's unconditional love, support, patience, and belief in me is my guiding light and gives me the courage to attempt all things. Love ya, Mo!

Finally, I owe every breath I take and my very being to my wife, Lauren. She has been there from the beginning, and her spirit and love have given me the strength to power through catastrophes that should have broken me. We did it. 


\section{Contents}

\begin{tabular}{|ll} 
Acknowledgments & iv
\end{tabular}

$\begin{array}{ll}\text { List of Figures } & \text { ix }\end{array}$

List of Tables $\quad$ xii

\begin{tabular}{lll}
\hline & Introduction & 1
\end{tabular}

1.1 Motivation . . . . . . . . . . . . . . . . . . . . . . 1

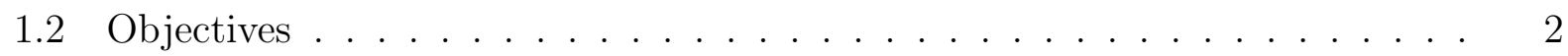

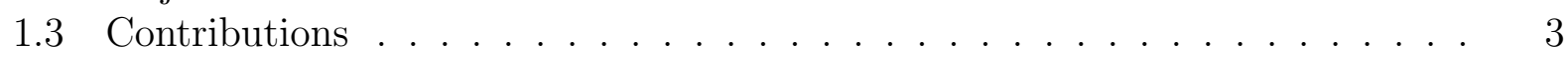

1.4 Dissertation Overview $\ldots \ldots \ldots \ldots \ldots \ldots$

\begin{tabular}{lll}
\hline 2 & Background & 7
\end{tabular}

2.1 Pulsar Astronomy Background . . . . . . . . . . . . . . . . . . . . . . 7

2.1 .1 Pulsar Discovery $\ldots \ldots \ldots \ldots \ldots$. . . . . . . . . . . . . . . . . . . . . . . . . . . . . . . .

2.1 .2 Radio Pulsar Data Sets . . . . . . . . . . . . . . . . . . . . . . . . . 15

2.2 Machine Learning Background . . . . . . . . . . . . . . . . . . . . . . 16

2.2 .1 Unsupervised Machine Learning Algorithms . . . . . . . . . . . . . . . . 17

2.2 .2 Supervised Machine Learning Algorithms . . . . . . . . . . . . . . . . 18

$2.2 .3 \quad$ Semi-Supervised Machine Learning . . . . . . . . . . . . . . . . . . . 23

2.2 .4 Metrics for Evaluation of Classification Performance. . . . . . . . . . 25

2.2 .5 Feature Selection . . . . . . . . . . . . . . . . 27

\begin{tabular}{|lll}
\hline 3 & Related Work & 30
\end{tabular}

3.1 Related Work on Periodicity Searches . . . . . . . . . . . . . . . . . . . . . . 30

3.2 Related Work on Single Pulse Searches . . . . . . . . . . . . . . . . . . . . . 33

3.3 Related Work on Peak Identification . . . . . . . . . . . . . . . . . . . . . . 35

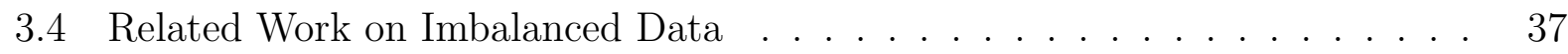

3.5 Related Work on High Performance Computing . . . . . . . . . . . . . . . . 38

3.6 Related Work on Random Forests . . . . . . . . . . . . . . . . . . . . . . . . 40

3.7 Related Work on Semi-Supervised Learning . . . . . . . . . . . . . . . . . . 44

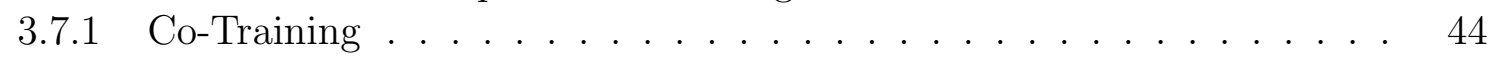

3.7 .2 Self-Training Random Forests . . . . . . . . . . . . . . . . . . . . 47

$3.7 .3 \quad$ Semi-Supervised Algorithms in Astronomy . . . . . . . . . . . . . . . 48 
4 Dispersed Pulse Group Identification \& Classification: Methods 52

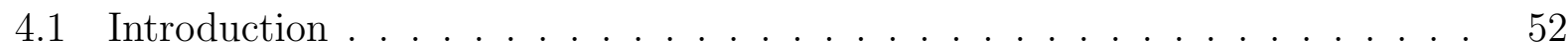

4.2 DPG Identification with RAPID . . . . . . . . . . . . . . . . . . . . . . . 55

$4.3 \quad$ Feature Extraction $\ldots \ldots \ldots \ldots \ldots \ldots$

$4.4 \quad$ DPG Classification with Machine Learning . . . . . . . . . . . . . . . . . 59

4.4 .1 Multiclass Classification . . . . . . . . . . . . . . . . . . . . 59

4.4 .2 Imbalance Considerations . . . . . . . . . . . . . . . . . . 60

4.4 .3 Learning Process . . . . . . . . . . . . . . . . . . . . . . . . . . . . . . . . . . . 62

4.5 Conclusions $\ldots \ldots \ldots \ldots \ldots \ldots$

5 Dispersed Pulse Group Identification \& Classification: Evaluation $\quad 64$

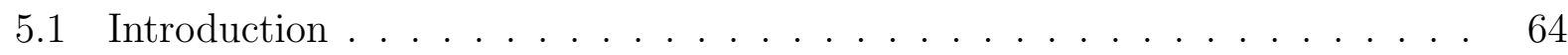

5.2 Data Pre-processing . . . . . . . . . . . . . . . . . . . . . 64

5.3 Creating the Benchmark Data Set . . . . . . . . . . . . . . . . . . . . 65

5.4 Results . . . . . . . . . . . . . . . . . . . . . . . 66

5.4 .1 Benchmark Data Set Results . . . . . . . . . . . . . . . . 66

5.4 .2 Full Data Set Results . . . . . . . . . . . . . . . . . . . 70

5.4 .3 Comparison of Our Results to Simple Ranking . . . . . . . . . . . . . 73

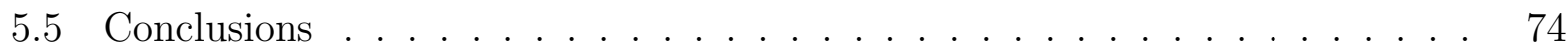

6 Single Pulse Event Identification \& Classification: Methods $\quad 76$

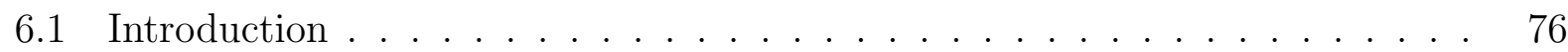

6.2 Feature Extraction . . . . . . . . . . . . . . . . . . . . . 80

6.3 Single Pulse Classification with Machine Learning . . . . . . . . . . . . . . . 81

6.3 .1 Imbalance Considerations . . . . . . . . . . . . . . . . . 81

6.3 .2 Multiclass Classification . . . . . . . . . . . . . . . . . . 82

6.3 .3 Feature Selection . . . . . . . . . . . . . . . . . 85

6.4 Conclusions . . . . . . . . . . . . . . . . . . . . . 86

\begin{tabular}{|lll}
\hline 7 & Single Pulse Event Identification \& Classification: Evaluation & 87
\end{tabular}

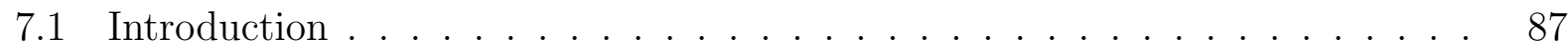

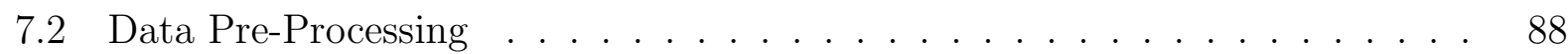

8.3 Creating the Benchmark Data Sets . . . . . . . . . . . . . . . . . . . . . 89

7.4 Results . . . . . . . . . . . . . . . . . . . . . . . . . . . . 89

7.4.1 Machine Learning Algorithm Results . . . . . . . . . . . . . . . . . . 90

7.4 .2 Imbalance Treatment Results _. . . . . . . . . . . . . . . . . . . . . . . . . . . . 91

7.4.3 Multiclass Classification Results . . . . . . . . . . . . . . . . . . . . . 92

7.4 .4 Feature Selection Results . . . . . . . . . . . . . . . . . 96

7.5 Conclusions $\ldots \ldots \ldots \ldots \ldots$

8 Distributed Single Pulse Event Identification: Methods \& Evaluation 101

8.1 Introduction . . . . . . . . . . . . . . . . . 101

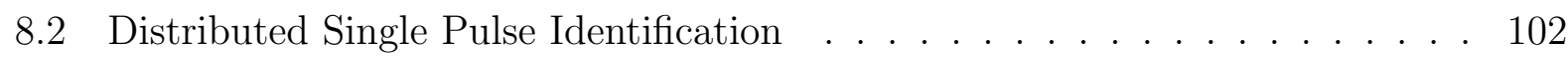

$8.2 .1 \quad$ Distributed Development Environment . . . . . . . . . . . . . . . . . 102

8.2 .2 D-RAPID . . . . . . . . . . . . . . . . 103

$8.2 .3 \quad$ Single Pulse Identification with D-RAPID $\ldots \ldots . . . . .106$ 
8.3 Results . . . . . . . . . . . . . . . . . . . . . . . . . . . . . . . 108

8.3 .1 Distributed Computing Environment . . . . . . . . . . . . . . 108

8.3 .2 D-RAPID Execution Performance Results . . . . . . . . . . . . . 109

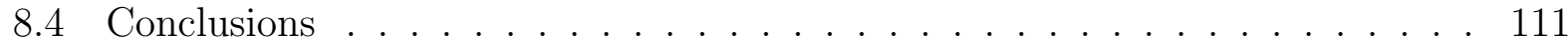

9 Distributed Single Pulse Event Classification with Semi-Supervised Learning: Methods \& Evaluation 112

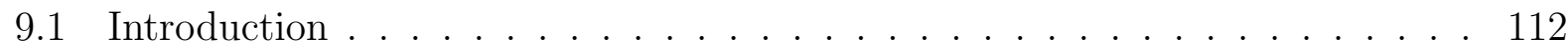

9.2 CoDRIFt . . . . . . . . . . . . . . . . . . . . . . . . . . . . . 114

9.3 Algorithms Used for Comparison $\ldots \ldots \ldots$

9.3 .1 Collective Classification . . . . . . . . . . . . . . . . . . . . . . 119

9.3 .2 SupervisedMLRF . . . . . . . . . . . . . . . . . . . . . . . . 121

9.3 .3 SelfLearningMLRF . . . . . . . . . . . . . . . . . . . . . . 122

9.4 Results . . . . . . . . . . . . . . . . . . . . . . . . . . . . . . . . 123

$9.4 .1 \quad$ Serial \& Distributed Computing Environments . . . . . . . . . . . . . 124

$9.4 .2 \quad$ CoDRIFt Results . . . . . . . . . . . . . . . . . . . . . . . . . . . 124

9.4 .3 Collective Classification Results . . . . . . . . . . . . . . . . . . 129

9.4 .4 SupervisedMLRF Results . . . . . . . . . . . . . . . . . . . . . 133

9.4 .5 SelfLearningMLRF Results . . . . . . . . . . . . . . . . . . . . . . 139

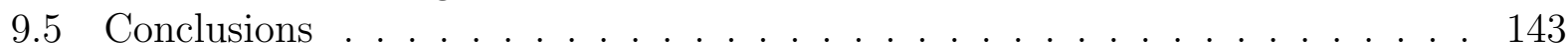

10 Conclusion \& Future Work 145

10.1 Motivation . . . . . . . . . . . . . . . . . . . . . . . . . . 145

10.2 Main Contributions . . . . . . . . . . . . . . . . . . . . . 146

10.3 Future Work . . . . . . . . . . . . . . . . . . . . . . . . 147

$\begin{array}{ll}\text { References } & 155\end{array}$ 


\section{List of Figures}

2.1 Pulsars are rapidly rotating neutron stars that emit radiation from their magnetic poles. Credit: B. Saxton, NRAO/AUI/NSF . . . . . . . . . . 8

2.2 A high-level data flow diagram. . . . . . . . . . . . . . . . . . . 11

2.3 The known pulsar J0354+0854 identified by a periodicity search. . . . . . . . 12

2.4 The known pulsar J0354+0854 identified by a single pulse search. . . . . . . 14

2.5 An example of a simple decision tree for determining if you have a "nice tuna"

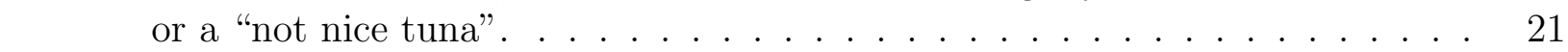

2.6 Confusion matrix for pulsar classification. The rows represent the predicted class value of the model, and the columns represent the actual values. . . . 26

4.1 Our new machine learning approach to singlepulse detection. . . . . . . . . . 54

4.2 The maximum SNR values (solid line) overlaid with the calculated fit (dashed line) according to Equation 4.1 for the DPG representing the known pulsar J1645-0317. . . . . . . . . . . . . . . . . . . 60

$4.3 \quad$ Four classes of pulsars based on the appearances of their candidate plots. . . 61

5.1 The results of binary machine learning trials on the four versions of the benchmark data set. The median of each distribution is the central horizontal line, the shaded areas to either side illustrate the semi-inter-quartile ranges, the lines or "whiskers" extending from each end give the first and fourth quartiles, and outliers are represented by open circles. The distributions of performance metrics (shown on the y axis) for each learner (annotated at the bottom) are organized into four groups, one for each version of the benchmark data set

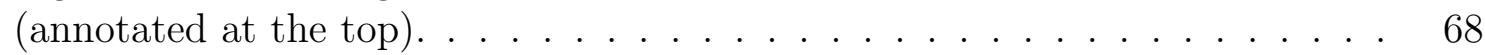

$5.2 \quad$ One potential RRAT discovery found by our classifiers. . . . . . . . . . . . 72

6.1 The known pulsar B1853+01 identified by a single pulse search. The SPEs emphasized in black belong to two distinct pulses. The subplots, clockwise from the top left, show a zoomed in scatter diagram of the SNRs for each SPE in the of DM range of the emphasized single pulse (in $\mathrm{pc} \mathrm{cm}^{-3}$ ), a scatter diagram showing the SNRs and DMs of all SPEs in a standardized DM range, and a scatter diagram showing the DMs and times (in seconds) of each SPE, with the markers for individual SPEs scaled in size by their SNR. . . . . . . 77

6.2 The scientific workflow of our scalable approach to single pulse identification and classification. . . . . . . . . . . . . . . . . . 79 
7.1 Bar charts showing the mean performance (left) and mean time efficiency (right) of six machine learning algorithms ( $x$-axis) organized by pulsar survey

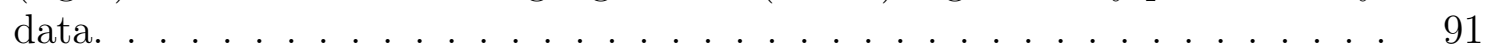

7.2 Two sets of bar charts showing the mean classification performance (left) and

\begin{tabular}{|c|c|c|}
\hline execution performance (right) of six machine learning algorithms. The charts \\
\hline \hline are subdivided into rows by survey and columns by imbalance treatment, with
\end{tabular}

\begin{tabular}{|l}
\hline execution performance (right) of six machine learning algorithms. The charts \\
\hline \hline are subdivided into rows by survey and columns by imbalance treatment, with
\end{tabular} "None" indicating no imbalance treatment.

7.3 Boxplots of the Recall, F-Measure scores for all classifiers subdivided by ALM scheme (columns) and data set (rows).

7.4 Boxplots of the training (left) and testing (right) times for all classifiers subdivided by ALM scheme (columns) and data set (rows). . . . . . . . . . . 95

7.5 Boxplots showing training times for RF (left) and MPN (right) classifiers. The x-axis shows the feature selection method and the subdivisions show the ALM scheme for the GBT350Drift (top row) and PALFA (bottom row) data sets. Note that the scales are different. . . . . . . . . . . . . . . . 97

7.6 Boxplots showing the ranks applied to each feature (on the $x$-axis) by the Info Gain feature selection technique. . . . . . . . . . . . . . . . . . 100

8.1 Control flow for a typical Spark job.

8.2 The stages of execution for D-RAPID to search a set of clusters for single pulses. 105

8.3 Performance evaluation of elapsed time to execute D-RAPID and a multi-

\begin{tabular}{|lll}
\hline threaded version (RAPID) on a 10.2 GB test set with $5,10,15$, and 20 \\
\hline \hline executors or threads, respectively. $\ldots \ldots \ldots$ & $\ldots \ldots \ldots$
\end{tabular}

\begin{tabular}{|l}
\hline threaded version (RAPID) on a 10.2 GB test set with $5,10,15$, and 20 \\
\hline \hline executors or threads, respectively. . . . . . . . . . . . . 110
\end{tabular}

9.1 Mean recall (top) and F-4 scores (bottom) scores for CoDRIFt models built

on training sets from the data for GBT350Drift and PALFA with the percent

of labeled instances ranging from $1 \%$ to $15 \%$. . . . . . . . . . . . . . . 126

9.2 Mean training (top) and testing (bottom) times for CoDRIFt models built on training sets from the data for GBT350Drift and PALFA with the percent of labeled instances ranging from $1 \%$ to $15 \%$. . . . . . . . . . . . . 128

9.3 Recall scores for nine Collective Classifiers (one per panel) built on training sets from the PALFA data with the percent of labeled instances ranging from

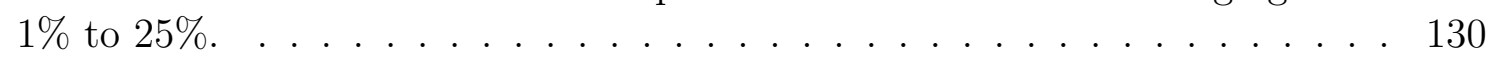

9.4 Recall (left) and F-Measure (right) scores for nine different semi-supervised learning classifiers on the PALFA binary benchmark with varying percentages

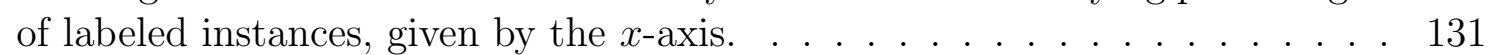

9.5 Training (top) and testing (bottom) times for nine Collective Classifiers (one per panel) built on training sets from the PALFA data with the percent of labeled instances ranging from $1 \%$ to $25 \%$. . . . . . . . . . . . . 132

9.6 F-4 scores for SupervisedMLRF models built on training sets ranging from $25 \%$ to $95 \%$ of the data for GBT350Drift (top) and PALFA (bottom). Each panel shows the scores for the training \% (top row of top label) and range of trees used (bottom row of top label). The $x$-axis shows the number of executors used for each group. . . . . . . . . . . . . . . . . . . 135 
9.7 Mean F-4 scores for SupervisedMLRF models built on training sets from the data for GBT350Drift (top) and PALFA (bottom) with the percent of labeled instances ranging from $1 \%$ to $95 \% . \ldots \ldots \ldots \ldots \ldots \ldots$

9.8 Mean recall (top) and F-4 (bottom) scores for SupervisedMLRF models built on training sets from the data for GBT350Drift and PALFA with the percent of labeled instances ranging from $1 \%$ to $15 \%$. . . . . . . . . . . . . . 137

9.9 Mean training times for SupervisedMLRF models built on training sets from the data for GBT350Drift and PALFA with the percent of labeled instances

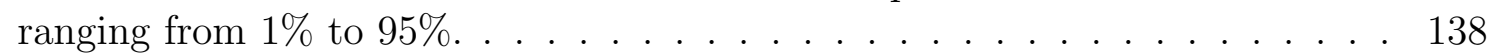

9.10 Mean recall (top) and F-4 (bottom) scores for SelfLearningMLRF models built on training sets from the data for GBT350Drift and PALFA with the percent of labeled instances ranging from $1 \%$ to $15 \%$. . . . . . . . . . . . . . . 141

9.11 Mean training (top) and testing (bottom) times for SelfLearningMLRF models built on training sets from the data for GBT350Drift and PALFA with the percent of labeled instances ranging from $1 \%$ to $15 \%$. . . . . . . . . . . . . 142

10.1 Boxplots showing Recall and F-Measure scores for all classifiers. The x-axis shows the training class and the subdivisions show the testing class. . . . . . 149

10.2 Boxplots showing training times for all classifiers. The x-axis shows the training class and the subdivisions show the testing class. . . . . . . . . . . . . 150

10.3 Boxplots showing testing times for all classifiers. The x-axis shows the training class and the subdivisions show the testing class. . . . . . . . . . . . . . . 151

10.4 Boxplots showing Recall and F-Measure scores for all classifiers. The x-axis shows the feature selection method and the subdivisions show the multiclass labeling scheme. . . . . . . . . . . . . . . . . . . 152

10.5 Boxplots showing training and testing times for all classifiers. The $\mathrm{x}$-axis shows the feature selection method and the subdivisions show the multiclass labeling scheme. . . . . . . . . . . . . . . . . . . . 153

10.6 Boxplots showing training and testing times for all classifiers. The $\mathrm{x}$-axis shows the feature selection method and the subdivisions show the multiclass labeling scheme. . . . . . . . . . . . . . . . . 154 


\section{List of Tables}

4.1 Features extracted for each DPG and used by machine learning algorithms for classification. Features $5-8$ were taken from data in the Pulse Counts vs DM plot in Figure 2.3, while the rest of the features were taken from the SNR vs DM plot in Figure 2.3 . . . . . . . . . . . . . . . . . . . . 58

4.2 The name and type of each machine learning algorithm used for this work. . $\quad 63$

5.1 The benchmark results for our classifiers. The center columns report mean values for the performance metrics described in Section 2.2 .4 . The final two columns report the average time taken to train and test the learners. . . . . 67

5.2 A comparison of the performance of classifiers using binary RandomForest (RF) and Multilayer Perceptron Network (MPN) learners on the full data set. 70

5.3 A comparison of the performance of oversampled and SMOTE multiclass RandomForest (RF) classifiers on the full data set. . . . . . . . . . . . . 71

5.4 Rankings based on a simple threshold for the feature $S N R M a x \chi^{2}$. The first column gives the statistic used as the threshold value, the second column shows the value of $S N R M a x \chi^{2}$ which will be used as the threshold, the third column displays the number of DPGs in the full data set that have a value below the threshold, the fourth column shows how many of the DPGs in the third column are known pulsars, the fifth column gives the percent of known pulsars detected below the given threshold, and the sixth column shows what percentage of the top ranked DPGs are pulsars (PER). For many DPGs, the fitting routine could not reach convergence. . . . . . . . . . . . 73

6.1 Summary information about the SPEs contained by the pulses emphasized in

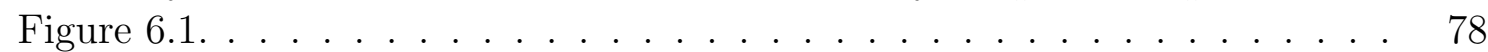

6.2 Additional features extracted for each cluster and used by machine learning algorithms for classification. . . . . . . . . . . . . . . . 80

6.3 The features and thresholds used to perform Automatically Labeled Multiclass

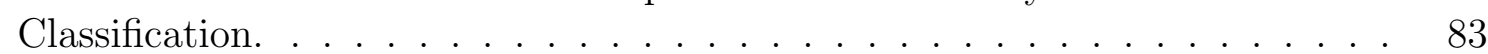

6.4 The five different multiclass classification schemes tested. . . . . . . . . . . . 84

6.5 The five different feature selection algorithms used. . . . . . . . . . . . . . 85 


\section{Chapter 1}

\section{Introduction}

Ever since Galileo Galilei first pierced the veil of the heavens and began to reveal the secrets of the celestial sphere, humans have turned their eyes and efforts skyward to try to understand their place in the Cosmos. Since that first crude instrument, science has refined the mechanics of astronomy to develop the largest and most sensitive instruments on Earth, which see far more than a mortal eye ever could. As our ability to perceive surpasses the limits of anatomy, so too must our ability to process the information perceived. This work focuses on harnessing computing technology to aid in the identification and classification of extraterrestrial, transient radio signals. Transient radio signals are relatively short bursts of electromagnetic radiation detected at radio frequencies. In particular, we are concerned with transient radio signals as expected from pulsars and revealed by single pulse searches.

\subsection{Motivation}

In computer science, machine learning algorithms are commonly applied to intelligently and automatically search data sets to find, in a more cost efficient way, patterns that can often be missed by manual inspection alone. In radio astronomy, massive data sets are commonly gathered via extremely sensitive observations and are manually inspected by trained individuals to identify known patterns related to certain celestial phenomena. The goal of the work in this dissertation was to develop a machine learning approach to efficiently search for transient signals in radio astronomy. While this may sound like a simple proposition in 
theory, in practice we encountered many challenges. Some of these challenges (missing and incorrect data, data bloat, imbalance, etc.) could be solved via traditional means, while others were unique to this endeavor and required unique solutions.

Traditionally, pulsars are discovered by manual inspection of the candidates produced by periodicity or single pulse searches. Manual inspection by domain experts, to some extent, will always be integral to the pulsar discovery process. However, automation of the majority of the process is vital for the future of radio astronomy. Next generation instruments, such as the distributed Square Kilometer Array (SKA) of a quarter of a million dual dipole antennas and 350 radio dishes [61, or the Five-hundred meter Aperture Spherical Telescope (FAST) [92 in China will have many more beams than the current instruments, resulting in significantly larger data sets. In addition to data volume, the speed at which data arrives is also increasing rapidly. Instruments and sensors are increasingly capable of generating data on the order of petabytes per second [98. Automated approaches are the only feasible way to deal with Big Data, and offer many potential advantages to streamline the discovery process, e.g., by triggering the rapid follow up of candidates at multiple wavelengths to constrain their origins.

While expert knowledge and manual inspection will always play a role in the pulsar search process, automated machine learning approaches, such as the ones presented in this dissertation, have great potential for future discoveries in radio astronomy. As radio astronomy technology advances, so must computing strategies for radio data processing and knowledge discovery. Intelligent, scalable search techniques are the only viable response to the astronomical data deluge on the horizon.

\subsection{Objectives}

The goal of the work presented in this dissertation is to provide scalable, machine learning solutions for pulsar candidate identification and classification in radio astronomy. The identification of candidates involves the development of methods to characterize the data generated by single pulse searches. This is a challenging task due to the the very large scale of radio data sets. Candidate identification is computationally expensive and must be 
repeated potentially millions of times over a single data set. Techniques for candidate identification must be scalable and implemented in parallel on distributed systems to efficiently process the astronomical amount of data received.

Candidate classification occurs after identification and is the process of training algorithms on known instances of pulsar and non-pulsar candidates so that they can accurately predict the labels (pulsar or non-pulsar) of unseen data. Several major challenges also exist for classification in this problem domain. First, pulsar candidates are extremely rare, which results in highly imbalanced training data sets. Many classification algorithms are not able to accurately handle imbalanced data, and will exhibit a strong bias for the majority class when making predictions on unseen data. Second, the process of labeling candidates for training sets is costly. Many hours must be spent by trained individuals sifting through candidate plots to create accurate labels, a process which may require additional telescope observation time to confirm pulsar candidates.

It is our hope that the algorithms, methods, and results presented in this dissertation will help alleviate some of the problems introduced by Big Data processing in radio astronomy.

\subsection{Contributions}

The main contributions of this work are algorithmic, methodological, and the provision of empirical evidence. These contributions are:

- We propose the first machine learning approach to pulsar candidate identification and classification in single pulse searches. The approach consists of two main stages: candidate identification using RAPID (see next bullet) and candidate classification using supervised machine learning.

- We propose RAPID, a novel, multithreaded algorithm for pulsar candidate identification. RAPID is designed to execute in parallel on a single workstation by taking advantage of multithreading to execute different instances on multiple CPU cores simultaneously. Since the data for each sky position are independent, RAPID can be instantiated in multiple threads to process the data from multiple positions simultane- 
ously. The output from each scan for DPGs is saved individually and the results are aggregated when all scans are completed.

- We propose ALM, a novel, automated multiclass labeling technique designed to facilitate the labeling of pulsar candidates. While all pulsar candidates are governed by the same physical laws, in the data we noticed that candidates appear different based on differing physical characteristics. Multiclass classification allows machine learning algorithms to become more specialized by taking advantage of similarities between candidates of one group to distinguish them from candidates of other groups.

- We propose D-RAPID, a software solution for searching very large radio data sets by leveraging automated data analytics and parallel data processing on distributed systems. We scaled-up a modified version of the identification algorithm we first presented in [34 to run in parallel using Apache Spark on a Hadoop YARN distributed system and show that it outperforms its multithreaded counterpart.

- We propose CoDRIFt, a novel, distributed algorithm for semi-supervised pulsar candidate classification. CoDRIFt is designed for parallel execution on distributed systems to address scalability concerns and uses semi-supervised learning to effectively train an ensemble of strong predictors from training data with very few labeled instances. CoDRIFt emphasizes construction of an ensemble of stronger learners, rather than training learners on larger data sets with more labels (which is common in the literature). CoDRIFt also combines supervised and semi-supervised learning by including predictors trained only on human-labeled data along with predictors trained on machine-labeled data in its ensemble. CoDRIFt uses a novel weighted voting scheme to improve performance imbalanced data sets and allows unlabeled instances to be labeled multiple times in different ways during training.

Furthermore, we conducted experimental evaluations of:

- six serial, supervised machine learning algorithms,

- three imbalance treatment methods, 
- the best and worst features for pulsar candidate classification,

- five feature selection algorithms,

- nine serial, semi-supervised machine learning algorithms,

- one distributed supervised machine learning algorithm, and

- one distributed semi-supervised machine learning algorithm.

We performed these evaluations using real-world radio astronomy pulsar surveys from the Green Bank Telescope and the Arecibo Observatory. We created fully labeled benchmark data sets for the data available from each survey to use in our experiments. Overall, this dissertation provides formal results from a grand total of 34,981 experimental trials.

Results of this dissertation are published in the astronomical journal Monthly Notices of the Royal Astronomical Society [34] and the 47th International Conference on Parallel Processing, a highly selective computer science conference [35].

\subsection{Dissertation Overview}

The remainder of this dissertation is organized as follows. Background information about radio pulsar astronomy and machine learning techniques is provided in Chapter 2. We review related works from the literature in Chapter 3. In Chapter 4, we provide details of our machine learning approach to pulsar candidate identification in dispersed pulse groups and present a novel, mutlithreaded pulsar candidate identification algorithm. Using the approach outlined in Chapter 4, we experimentally evaluate six machine learning algorithms and three imbalance treatments for dispersed pulse group classification on real-world radio astronomy data in Chapter 5. In Chapter 6, we present a machine learning methodology for single pulse candidate identification and classification, and introduce a novel multiclass labeling technique. We report the results of experimental testing of this approach in Chapter 7, including the evaluation of six machine learning algorithms and five feature selection techniques. In Chapter 8, we present a distributed parallel implementation and refinement of the algorithm introduced in Chapter 4, along with an experimental evaluation of its 
performance. In Chapter 9, we explore the use of existing semi-supervised machine learning algorithms for the problem of pulsar candidate classification and propose a novel, distributed, semi-supervised algorithm. We evaluate our algorithm by comparing it to existing serial and distributed supervised and semi-supervised algorithms. Chapter 10 presents our concluding remarks and the focus of future work. 


\section{Chapter 2}

\section{Background}

In this chapter, we provide background information about radio pulsar astronomy and machine learning. The methods and techniques described are not intended to be exhaustive, but contain the necessary information to understand the context and work of this dissertation.

\subsection{Pulsar Astronomy Background}

Pulsars, to the best of our ability to explain them, are rapidly spinning, extremely dense neutron stars which emit radio (among other forms of) radiation from their magnetic poles [81]. As depicted in Figure 2.1, due to the rapid spinning of the star, the emissions from the poles are seen from Earth as faint "pulses" of energy with extremely precise periods. These phenomena are comparable to the bright pulses of light that can be seen from the spinning beacon of a lighthouse at night. Rotating radio transients (RRATs), are thought to be a special type of sporadically emitting pulsar [89]. Throughout this dissertation, we will use the term 'pulsar' to describe pulsars and RRATs.

Pulsar astronomy, though a well-studied field for fifty years, has yet to answer many fundamental questions about these enigmatic celestial objects. While their true nature is elusive, two things are certain concerning their place in astrophysics: they are invaluable tools for understanding our Universe, and more pulsar discoveries lead to more powerful insights into the Cosmos. While the study of pulsars directly provides information about 
the extreme physics of neutron stars, their unique properties and variety of incarnations also provide tools for studying a wide range of scientific interests.

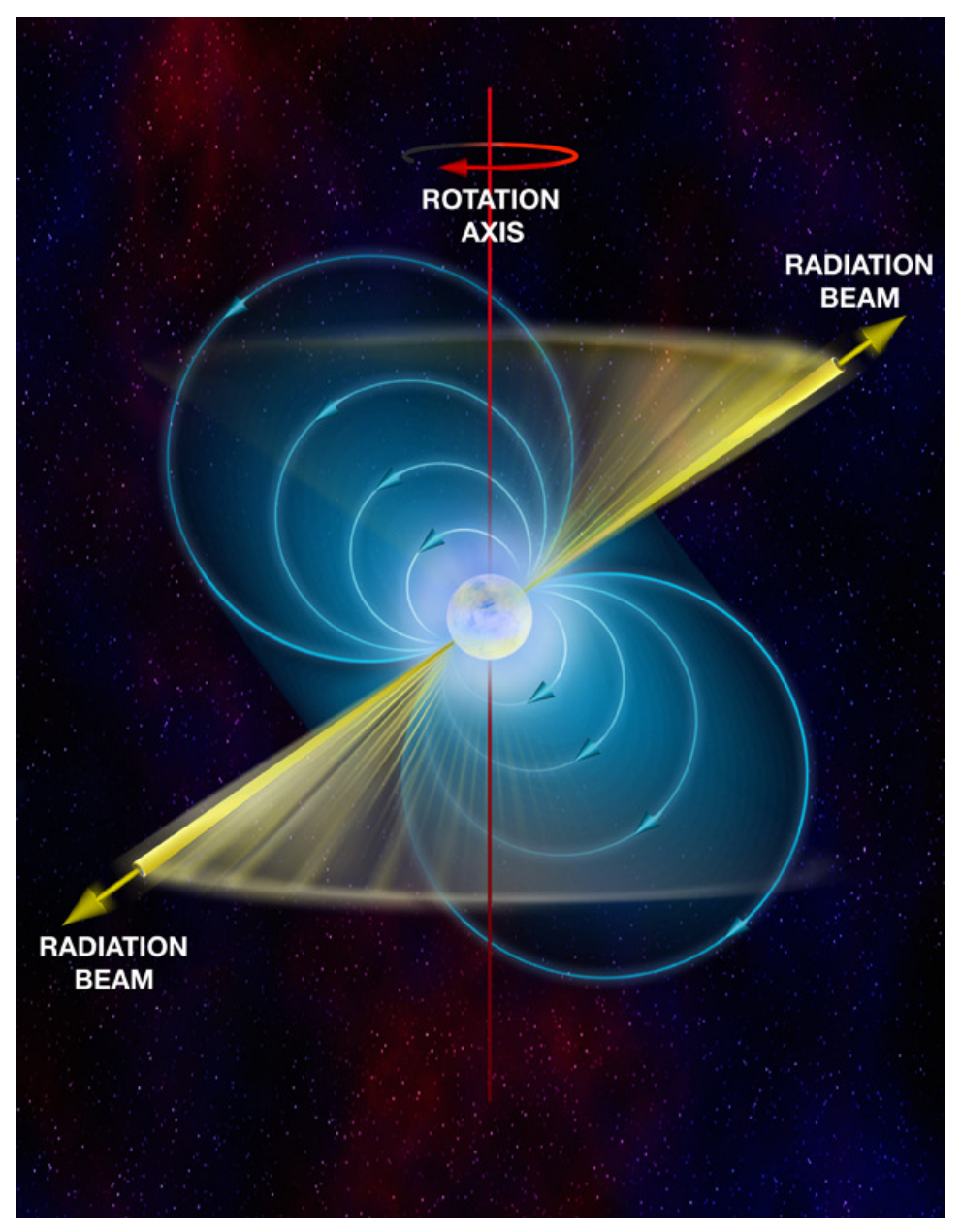

Figure 2.1: Pulsars are rapidly rotating neutron stars that emit radiation from their magnetic poles. Credit: B. Saxton, NRAO/AUI/NSF

Many different types of pulsars exist. They all have extreme densities, on the order of the mass of the Sun condensed to a 20 kilometer radius. However, the periods of their rotation can vary dramatically from one pulsar to another. The slowest known rotating pulsar has a period of 23 seconds, and the fastest can make a complete rotation in 1.4 milliseconds. The rotations of bright millisecond pulsars are extremely regular due to the large amount of rotational kinetic energy they possess combined with a relatively small energy loss over time. This makes them effective celestial timepieces that can, over long periods, approach the stability and precision of the best atomic clocks [81]. Pulsar timing observations also result 
in highly accurate determinations of their locations. These precise locations can be tracked and mapped for different purposes, including determination of the physical properties of the globular clusters they sometimes inhabit [81].

Pulsars with companions are also powerful extraterrestrial tools for testing gravitational theories. The rates of decrease in the orbital periods of double neutron star binaries have been used to provide evidence of general relativity's predicted gravitational radiation [81]. Precise measurements of millisecond pulsars in pulsar timing arrays have been shown to provide constraints on low-frequency gravitational waves that provide complementary information to detections at higher-frequencies by ground-based interferometers [120]. The discovery of more millisecond pulsars is anticipated to help provide stronger evidence of gravitational waves. Pulsar discovery, however, is not a trivial problem.

Pulsar discoveries have been made through a variety of techniques. Despite all of these discoveries, it has been theorized that the over 2,500 known pulsars comprise a small percentage of the potentially detectable pulsars in our galaxy [43]. Furthermore, additional pulsars may be detectable in globular clusters [5]. Discovering these pulsars, however, is very challenging. The signals are faint, requiring sensitive observations. Searches must deal with issues such as noise (resulting from receivers and the sky), radio frequency interference (RFI), and highly imbalanced data sets (i.e., only a very small fraction of the radio signals received originate from pulsars).

\subsubsection{Pulsar Discovery}

The pulsar phenomenon was initially discovered in 1968 via a fortunate visual inspection of a radio telescope's total power at high time resolution by Antony Hewish and Jocelyn Bell [56]. Since then, radio astronomers have been driven by necessity to develop extremely sensitive tools to gather photons from distant radio pulsar sources. As an example of how faint the signals received from radio pulsars are, the amount of energy gathered by the Arecibo Observatory's 100 meter radio telescope from all of the pulsars it studies in the course of a year is approximately the same as that required to print a single page of paper on a standard printer. Taking such sensitive measurements through the atmosphere of Earth, 
which is rife with radio frequency communication and interference, results in some of the noisiest data in the world. The weak signals of new radio pulsar discoveries lie hidden in the noise.

Pulsar discovery in radio data sets was traditionally approached in four main phases: collection, dedispersion, periodicity or single pulse search, and manual inspection. Figure 2.2服 provides a high-level depiction of how a signal is received and transformed into the input used by our methods. In the first phase, raw data are collected at radio telescopes as a time-series of voltages. Radio emissions travel through the interstellar medium (ISM) and are received by a radio telescope as a time series of raw voltages. These raw voltages are amplified by a low-noise amplifier, passed through a bandpass filter, and (usually) converted to a lower frequency for transmission efficiency and hardware compatibility according to the Signal Path given in Figure 2.2 [81].

The second and third phases are carried out using the software package PRESTO [105]. After some additional processing, the signals must be dedispersed in the second major phase. As a pulsar's radiation propagates through the ISM, the ISM causes the pulses to be dispersed, with lower frequency components of pulses arriving later than higher frequency components. The time delay between two frequencies depends on three things: the difference between the frequencies of the observations, the observational frequency, and the DM, which is the integrated number of free electrons along the line of sight measured in pc $\mathrm{cm}^{-3}$. The delay effectively "smears" the emissions across the time domain, reducing their signal-to-noise ratios (SNRs). Incoherent dedispersion is the process of removing these frequency-dependent delays via application of an artificial time delay in the reverse direction of the dispersion, aligning the pulse's energy in a narrow, vertical time slice. This signal is then integrated across the measured frequency band to reveal a strong single pulse. However, since the degree of dispersion for an unknown pulsar cannot be known a priori, many DMs must be tried. Incoherent dedispersion at many trial DM values results in a dedispersed time series, or DM channel, for each DM value [81].

\footnotetext{
${ }^{1}$ By NASA/CXC/PSU/G.Pavlov et al. - http://heasarc.gsfc.nasa.gov/docs/objects/heapow/ archive/compact_objects/vela_pulsar_jet.html

${ }^{2}$ By Scott Ransom - https://www.cv.nrao.edu/ sransom/PRESTO_search_tutorial.pdf
} 


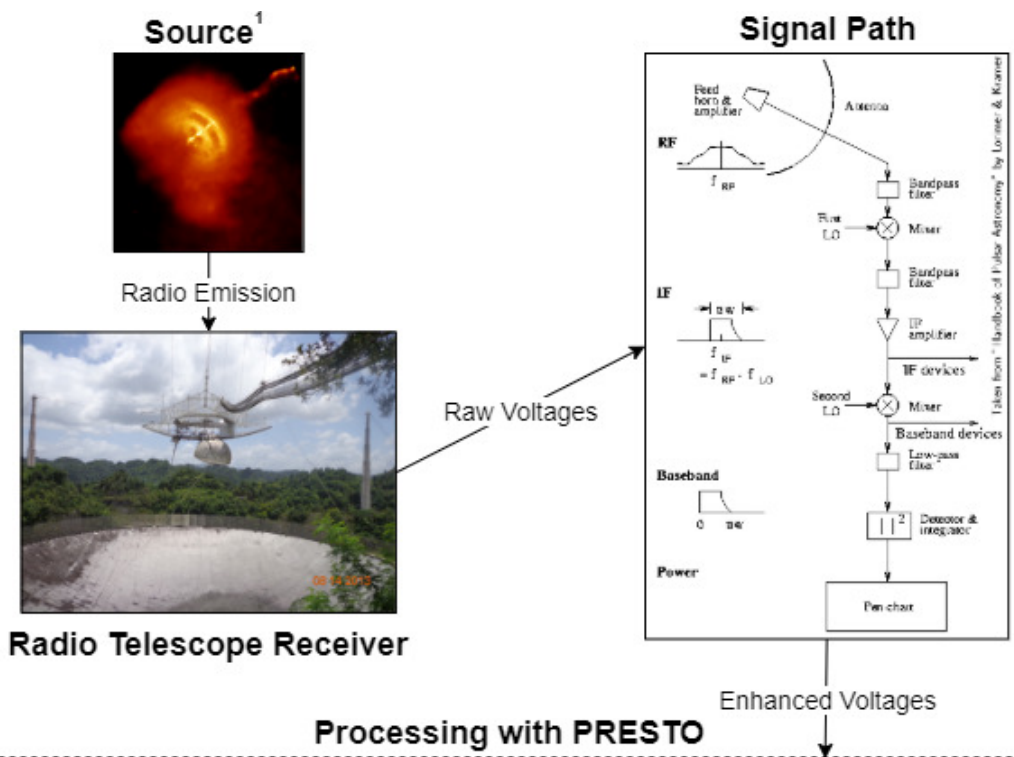

Outline of a PRESTO Search ${ }^{2}$

1) Examine data format (readifie)

2) Search for RFI ( $r \in i f i n d)$

3) Make a topocentric, DM=0 time series (prepciata and exploredac)

4) FFT the time series (realfft)

5) Identify "birdies' to zap in saarches (exploreffth

6) Make zaplst (makezaplist.py\}

7) Make De-cspersion plan (Doplan.

8) De-disperse (prepsulbiasd)

9) Search the data for periodic signals (accels saych)

10) Search the data for single pulses (single_pulse_seazch. py

11) Sitt through the candidates (ACCEL_sift.py)

12) Fold the best candidates ( $p x a p f o l d)$

13) Start timing the new pulsar (p zepptold and get__CAas.py)

Searching for Transient Bursts ${ }^{2}$

> single_pulse_search.py *.dat

- singlo_pulse_search. py conducts matched-fitering single.

- - fast can make things about a factor of 2 faster, but only use it $f$ the data are well-behaved (relatively constant power levels)

- If there are very strong pulses in your data, they can look like RFI.

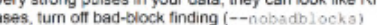

- Generates " singiepulse files that are ASCII and a single-pulse plot

- Can regenerate a plot using (for instance)

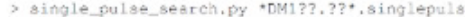

- Can choose start and end times as well (--start and --end)
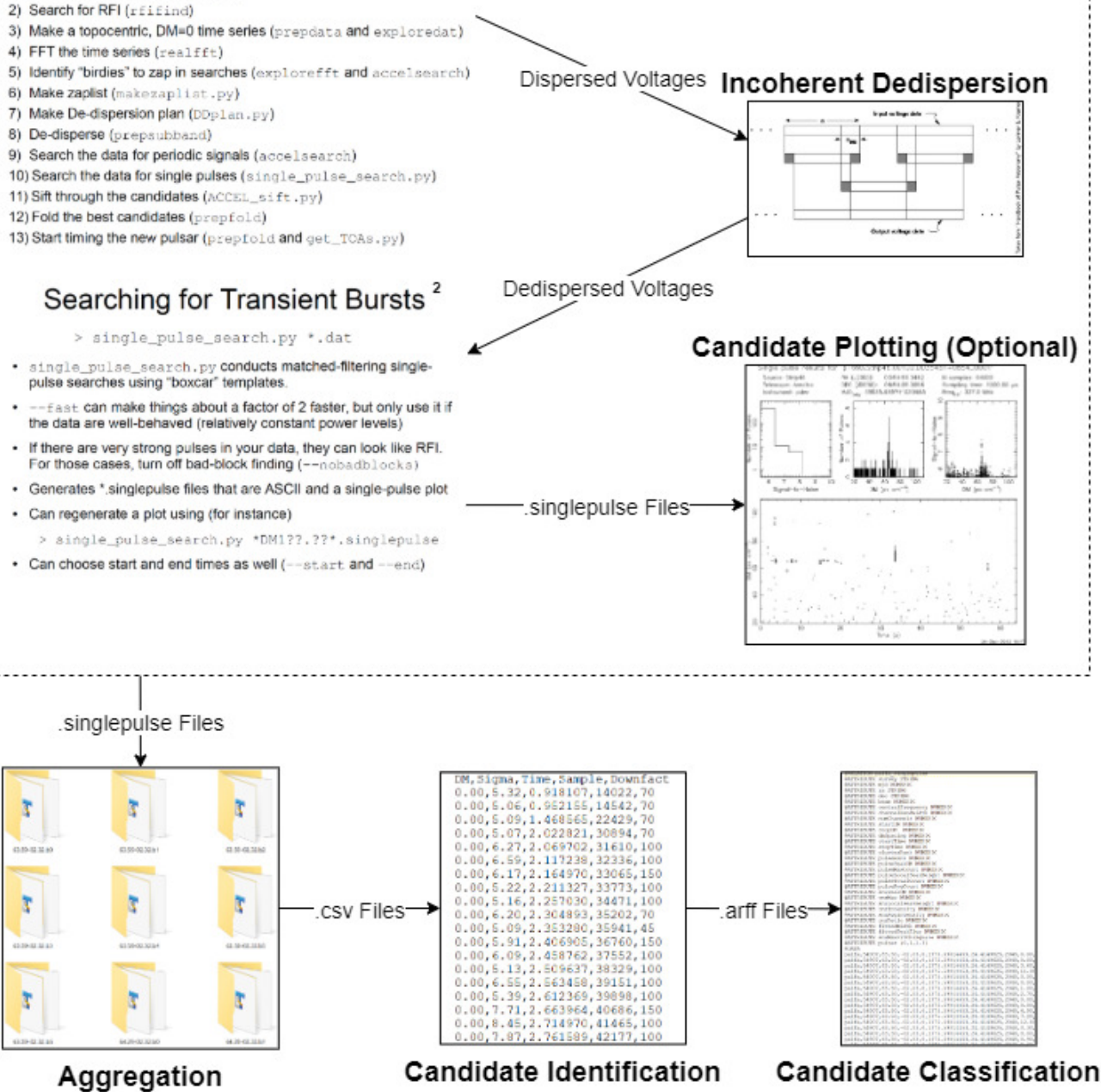

Figure 2.2: A high-level data flow diagram. 
In the third phase, PRESTO is used to conduct periodicity or single pulse searches over the DM channels. The two searches differ in the types of phenomena they attempt to detect. Periodicity searches transform the time series into the frequency domain by applying Fast Fourier Transforms (FFTs) to make regular, periodic signals stand out. The original time series data are then folded at the identified periods to amplify the SNRs of periodic signals [73. Figure 2.3 provides an example plot created from a periodicity search. Many works in the literature on radio pulsar searching leverage image recognition technologies to perform classification based on periodicity plots (see Section 3.1).

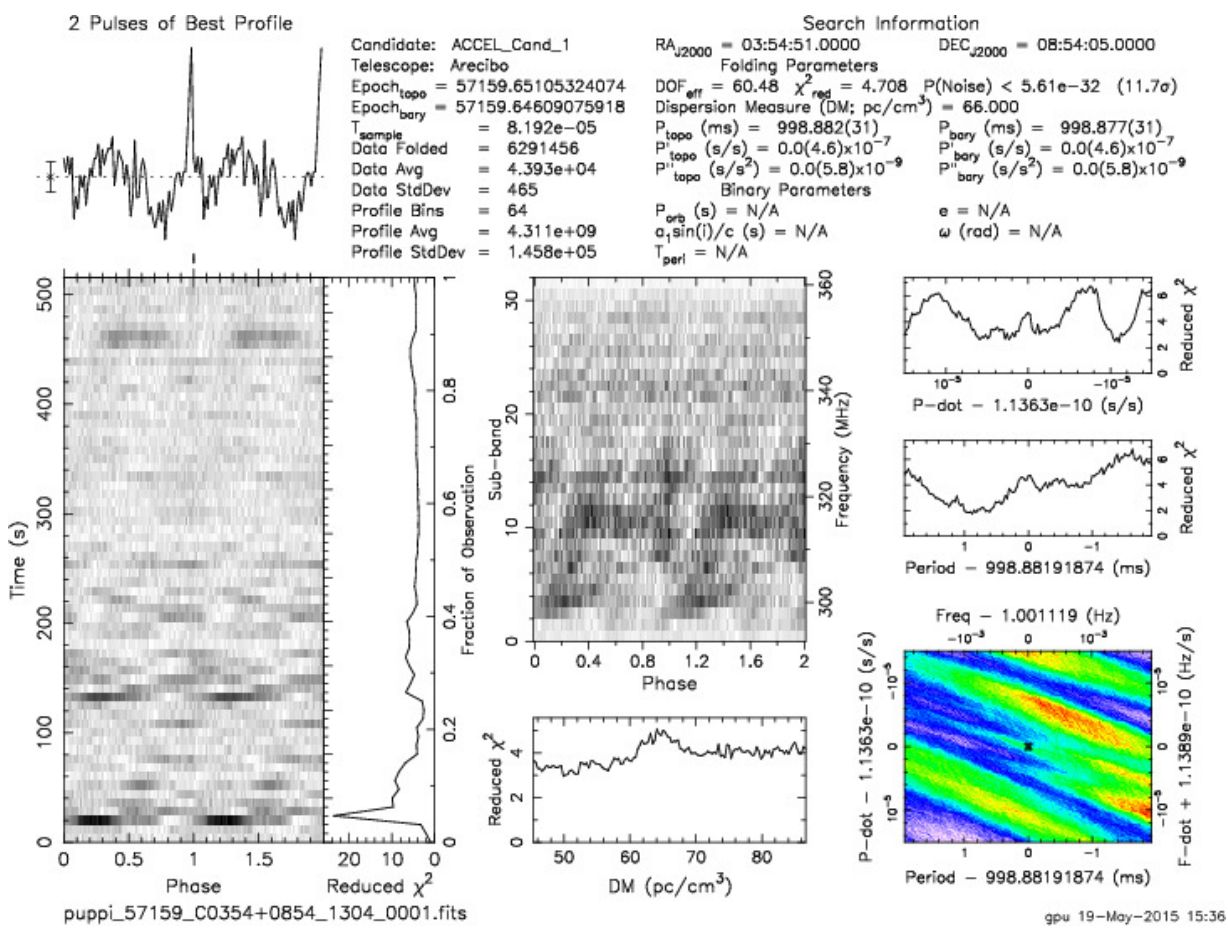

Figure 2.3: The known pulsar J0354+0854 identified by a periodicity search.

Single pulse searches, on the other hand, do not use FFTs or fold the data. This has the advantage of being able to detect strong, non-periodic signals that periodicity searches cannot detect. RRATs, for instance, are only detectable through their isolated pulses. However, single pulse searches typically are not able to detect very regular, weak signals that would show up clearly in a periodicity search. A single pulse search works by searching each DM channel for pulses with amplitudes above some given SNR threshold (five, in our data). The

\footnotetext{
${ }^{3}$ By Julia Deneva - http://www.naic.edu/ deneva/drift-search/puppi_57159_C0354+0854_1304_ 0001_ACCEL_Cand_1.pfd.png
} 
search itself is an exercise in matched filtering, where the SNR will reach its maximum when the effective sampling time of the time series closely matches the width of the pulse. For a real pulsar signal at its actual DM, the smearing effect of dispersion will be minimized resulting in a large SNR. In adjacent channels with incorrect DMs, however, the SNR will be lower due to increased smearing. This means that a single pulse is usually detected over more than one DM channel [29].

The fourth and final phase traditionally consisted of manually inspecting periodicity plots or single pulse plots generated by PRESTO. As the volume of data generated by these search techniques has increased over time, automated and machine learning approaches have been increasingly tried to reduce this reliance on manual inspection (see Chapter 3 for a thorough review of the literature).

For our work in this dissertation, we focused on the final two steps in Figure 2.2, candidate identification and candidate classification, where candidates are either pulsars or non-pulsars (RFI that look similar to pulsars). We used as our input the singlepulse files generated by PRESTO's single pulse searching routine. The .singlepulse files consisted of individual files for each DM channel searched. Each file is an ASCII text list of signals for a particular DM as described by their calculated SNR, downsampling factor, and time received. The Candidate Identification image (bottom middle of Figure 2.2) provides a small excerpt of the data contained in a .singlepulse file after aggregation into a single .csv file. While these data can be used to create single pulse candidate plots, such as the one in Figure 2.4 none of our approaches presented in this dissertation used the plots as input, i.e., our work does not rely on image processing of the plots. Instead, we wrote code to pre-process the .singlepulse files, reorganizing and transforming them as described in Sections 5.2 and 7.2 , and then identified candidates and extracted features for classification via machine learning.

Even though our approaches do not use single pulse search plots directly, they are still invaluable tools for the visualization and interpretation of single pulse search data and the discovery of pulsars with transient signals. While a periodicity plot shows results for one DM and period, single pulse plots show results from many different DM trial values in the same

${ }^{4}$ By Julia Deneva - http://www.naic.edu/ deneva/drift-search/p1693.Strip46.00100.D035451+ 0854_0001_DMs20-110_singlepulse.png 

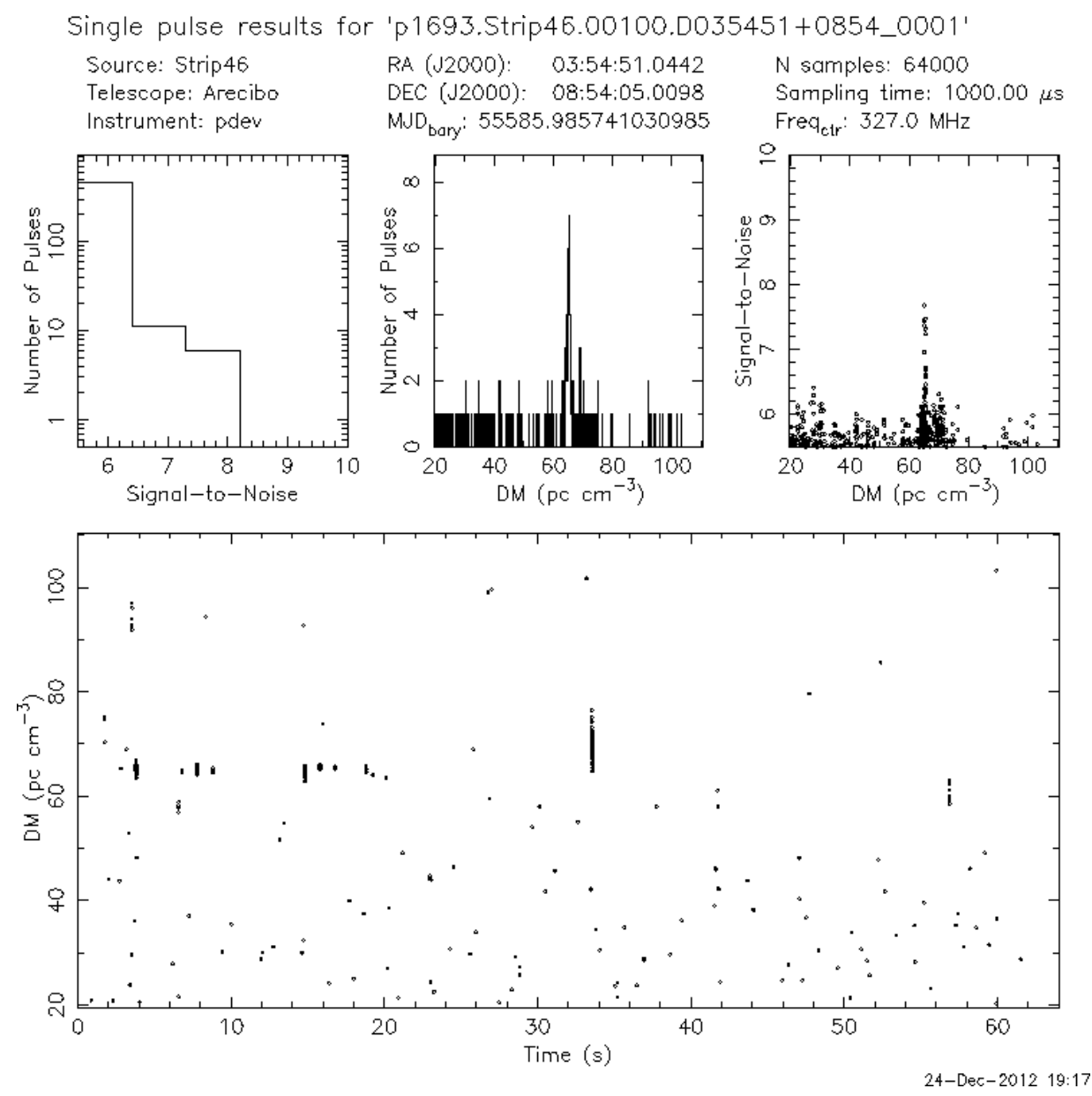

Figure 2.4: The known pulsar J0354+0854 identified by a single pulse search.

plot. A single pulse plot, as in Figure 2.4, contains four subplots: the top left is a histogram of the number of pulses for each SNR value, the top middle is a histogram of the number of observed signals for each DM, the top right is a scatter diagram showing the SNR values of any recorded pulses for each DM, and the bottom is a scatter diagram which shows the DM on the y-axis and the time each signal was recorded on the x-axis. In the bottom subplot, each point's size is scaled by the magnitude of its SNR value, i.e., larger SNR values appear as larger points.

The known pulsar J0354+0854, shown in Figure 2.4 and discovered by methods presented in this dissertation, can be identified by a number of telling features from its single pulse plot.

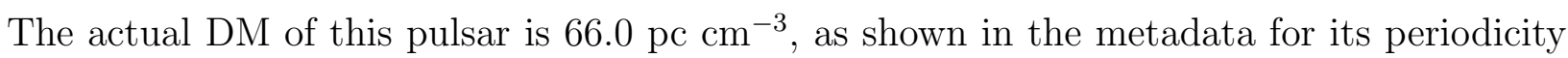
plot in Figure 2.3. In the single pulse plot, both the pulse histogram by DM and the SNR 
by DM subplots show a peak that occurs when the DM is $66.0 \mathrm{pc} \mathrm{cm}^{-3}$ and a falloff in both number of pulses and SNR in the adjacent DM channels. This occurs because the single pulse search achieves the best fit at that DM (resulting in the highest SNRs and the most pulses), and the fit degrades as the trial DM deviates from the correct value. In the bottom plot, the intermittent signaling of the pulsar is evident by the several groups of pulses with peaks aligned in the same DM channel.

\subsubsection{Radio Pulsar Data Sets}

This section contains a brief description of relevant pulsar surveys. While many more surveys exist in the literature, only those surveys involving data analyzed either by our methods or by works referenced in this dissertation are listed below.

The Parkes Multi-beam Pulsar Survey (PMPS) was completed between 1997 and 2003 using the Parkes radio telescope in Australia. The PMPS had a bandwidth of $1.374 \mathrm{GHz}$ and 3,000 channels with observation lengths of 250 seconds and resulted in the production of over eight million candidates [88].

The Southern and Northern High Time Resolution Universe (HTRU) pulsar and fast transient surveys were also performed with the Parkes radio telescope, but with four times the time resolution and eight times the frequency resolution of the PMPS. The Southern/ Northern HTRU surveys ran from 2010 through 2014 with central frequencies of 1.352/1.360 $\mathrm{GHz}$, bandwidths of 340/240 MHz, with observation times ranging from 90 to 4,300 seconds. When combined, the HTRU surveys resulted in over 135 million total candidates [6, 68,

The seven beam Pulsar Arecibo L-band Feed Array (PALFA) survey was a long-term pulsar survey of the Galactic plane which, for our data, ran from March 2009 through May 2015. PALFA used the Arecibo Observatory's L-band Feed Array with a seven-beam receiver operating at $1.420 \mathrm{GHz}$ with a $300 \mathrm{MHz}$ bandwidth, 1,024 channels, and observation times of 268 seconds for the inner Galaxy pointings and 180 seconds for the outer Galaxy pointings. The PALFA survey resulted in over five million candidates [28, 74].

The Green Bank Telescope (GBT) 350-MHz Drift-scan survey (GBT350Drift) was performed with the GBT from May through August in 2007. The survey was conducted while 
the GBT was immobilized for refurbishing. The receivers remained active throughout the repairs and collected data at a radio frequency of $350 \mathrm{MHz}$ as the sky passed through the beam of the telescope [15].

The LOFAR Tied-Array All-sky Survey (LOTAAS) was a high-band antenna survey of the northern sky at a central observing frequency of $135 \mathrm{MHz}$ started in 2013. LOTAAS covered 2,592 channels from 119 to $151 \mathrm{MHz}$ with each observation integrated for one hour, resulting in 39 million candidates [110].

\subsection{Machine Learning Background}

Machine learning is the process of using computers to automate (fully or partially) the discovery of meaningful patterns in large quantities of data [124]. In general, the data used to "train" an algorithm to discover patterns must be organized as a list of instances (or observations) which conform to a prescribed set of features (or attributes) and have a class value (or label). For example, if we wanted to train an algorithm to classify fish in the sea by the labels "nice tuna" or "not nice tuna", we would first need to select a sequence of measurable features to distinguish individual fish from each other. Such features could include length, weight, fat content, and any number of other definable fish features. Then, when we catch a new fish, we could feed its feature values into our algorithm, which would compare it to the patterns it has learned and tell us if we had a nice tuna to take to market.

Algorithms which perform machine learning can be divided into three categories based on the nature of the data they analyze: unsupervised, supervised, and semi-supervised. Unsupervised algorithms are used to identify groups of similar data when the class value is not known. Supervised algorithms make use of labeled training data to predict the value of unseen, unlabeled data. Semi-supervised algorithms use some combination of labeled and unlabeled data during training.

According to the "No Free Lunch" theorem for machine learning, there is no one algorithm that works best for every problem domain [125]. This is because most machine learning algorithms make assumptions about the relationships between the class value and the features. The effectiveness of a model in a particular problem domain directly depends 
on how well the assumptions for a particular model fit the true nature of the data. For this reason, it is common to test many different types of machine learning algorithms on a problem domain, as some will naturally outperform others for that specific domain. While some models (like simple rule learners) have high interpretability, other models (like neural networks) do not and are treated as "black boxes". However, restricting machine learning to interpretable models can be an unnecessary and severe limitation, as a black box model may significantly outperform models with more interpretability, and vice versa [107].

\subsubsection{Unsupervised Machine Learning Algorithms}

Unsupervised learning occurs when class labels are unknown. In the absence of known labels, unsupervised algorithms generally group together instances that are "similar" to each other. The most common unsupervised learning technique is a process called clustering [53]. Clustering algorithms vary based on how they define similarity between instances and how they create and organize the groups they identify.

The $k$-means clustering algorithm is, perhaps, the simplest algorithm to follow and begins by selecting a value for $k$ (this value will be the number of clusters the algorithm identifies). For a set of instances, $k$-means first selects $k$ random instances and treats them as the centers of $k$ clusters. Every instance is then assigned to the nearest cluster center according to Euclidean distance. Once all points are assigned, the centroid of each cluster is calculated as the average (mean) of all points assigned to the cluster. The centroids are then considered the centers of the clusters for the next round [124].

Another common clustering algorithm is Density-Based Spatial Clustering of Applications with Noise (DBSCAN) [42]. DBSCAN operates by identifying core objects and their neighborhoods. A core object and the neighborhood around it must contain enough instances to be considered densely populated, as specified by a user-selected threshold. Every instance inside the neighborhood of a core object is considered to be in the same cluster, and if the neighborhoods of two core objects overlap, they are joined together to form one single cluster. All instances that are not dense enough to be considered core objects or are not in the neighborhood of another core object are considered noise. 


\subsubsection{Supervised Machine Learning Algorithms}

Supervised learning uses known positive instances (in our case, pulsars) to build a classification model. Throughout this dissertation, we used six supervised machine learning algorithms of different types: an artificial neural network, support vector machine, direct rule learner, standard tree learner, hybrid rule-and-tree learner, and ensemble tree learner. The intent of choosing different types of learners was to see if any certain machine learning technique performs better overall when searching for pulsars in singlepulse search results. For this work, we used implementations available through Weka, a popular machine learning software suite [51].

Artificial neural networks (ANNs) have been used in several related papers working with periodicity searches, as mentioned in Section 3.1. ANNs are modeled after the way synapses work in the brain [109]. An ANN consists of a number of nodes organized in layers. Layers can be either input layers, output layers, or hidden layers. Nodes in one layer are connected to nodes in other layers by links, which represent the synapses between neurons in the brain. The links have weights, which are adjusted as information flows through the network. Throughout the training phase, specific paths through the network gain weight, representing the way synaptic pathways in the brain are reinforced by repeated traversal. The other key component of an ANN is the activation function. When the weighted inputs to an inner layer node are aggregated, they are then transformed by the activation function to determine the output of that node. Generally, non-linear activation functions, such as the sigmoid or tanh function, are used to allow for more complex, non-linear decision boundaries. The ANN we used is the Java implementation of a Multilayer Perceptron Network (MPN), which classifies instances using the supervised learning method of back-propagation and a sigmoid activation function in all neural nodes [12]. Back-propagation is the process of using a loss function to adjust the weights of incorrect classification paths after data has been fed through an ANN.

Several of the related works discussed in Sections 3.1 and 3.7.3 use Convolutional Neural Networks (CNNs) for image classification. A deep CNN is characterized by more than five hidden layers with convolution layers and max-pooled layers. While the output of each layer of an ANN is a linear combination of weighted inputs, the output of convolution layers are 
two-dimensional fields called "feature maps". A convolution layer has three parts: 1) the input image (receptive field), 2) convolutional filter, and 3) output feature map. A feature map is created by applying a convolutional filter over the input image in a sliding window, and its size is determined by size of input image, the sliding window, and the convolutional filter size. Pooling layers down-sample feature maps to smaller maps by taking four inputs and computing a maximum or mean [49].

Support Vector Machines (SVMs) are a class of supervised learners which create higher order decision boundaries, called hyperplanes, to separate different instances by class. They use mapping functions, called kernels, to transform the input space into a more easily separable feature space. To construct an optimal hyperplane to separate the instances in this transformed space, SVMs use iterative training algorithms to minimize an error function. Sequential minimal optimization (SMO) is a Java implementation of a support vector machine [100, 66]. SMO solves the optimization problem of minimizing error by a divide and conquer strategy, breaking the problem into a series of smallest possible problems which are then solved analytically.

The direct rule learner tested was JRip, the Java implementation of the RIPPER [27]. As a rule learner, JRip creates a set of rules from the training set and then classifies each instance in the test set based on the generated rules. The rules consist of one or more antecedents followed by a single consequent, following a basic "if antecedent(s) then consequent" structure. Rule learners follow a "separate and conquer" methodology, i.e., they build a rule that covers as many instances as possible, remove all instances for which that rule is true from the training set, then continue this process recursively until all instances are covered by at least one rule.

To generate more accurate rules, JRip follows an incremental, reduced-error pruning scheme with global optimization. Pruning, which is the removal of extraneous or inefficient rules, takes place by partitioning the training data into two sets, a growing set and a pruning set. A rule is grown from the growing set by greedily adding antecedents until the rule is one hundred percent accurate. The newly grown rule is then immediately pruned by deleting an antecedent from the rule and then evaluating its performance on the pruning set. The rule's performance is evaluated by treating the rule as the only judge in the pruning set and 
calculating the following heuristic:

$$
\frac{p-n}{p+n}
$$

where $p$ is the number of correctly classified instances and $n$ is the number of incorrectly classified instances. The pruning is continued until the rule's performance is no longer increased by the removal of antecedents. Once a set of rules is generated in this manner, the set is optimized by generating two variants of each rule, one from an empty rule and one by greedily adding antecedents to the original rule. The same pruning process is used on the two new rules, except the following metric is used in place of the previous:

$$
\frac{T P+P N}{P+N} \text {. }
$$

In this version, $P$ and $N$ are the total number of positive and negative examples of the given class in the pruning set, respectively, and $T=P+N$. Note that in this case, the totals are measured, not the number of instances covered by the rule.

A tree is a generic data structure which consists of nodes, branches, and leaves. The initial node is called the "root". From the root are branches to one or more child nodes, each with branches to their own children, and so on. Nodes with parents but no children are called "leaf nodes" or "leaves". In a decision tree, each node represents a feature in the training data. Branches from these nodes represent different conditions, or possible values, of the feature. For any branch, if all the instances from the training set which satisfy that branch's condition belong to the same class, then that branch is terminated by a leaf node which contains that class. If two classes exist in the set of instances satisfying the condition, then the branch continues to a new node, where a new feature must be selected and the process continued [124]. This process is continued on each node until every instance in the training set is covered by some decision path from root to leaf. Figure 2.5 provides a simple illustration of a decision tree to determine if a new fish caught is a "nice tuna" or "not nice tuna".

The standard tree learner we tested was J48, the Java implementation of C4.5 [103]. Decision tree algorithms approach classification with a "divide-and-conquer" strategy. They operate by determining what criteria best divides the test set into separate groups. J48 uses a normalized function called Info Gain, which is defined in terms of information content, or 


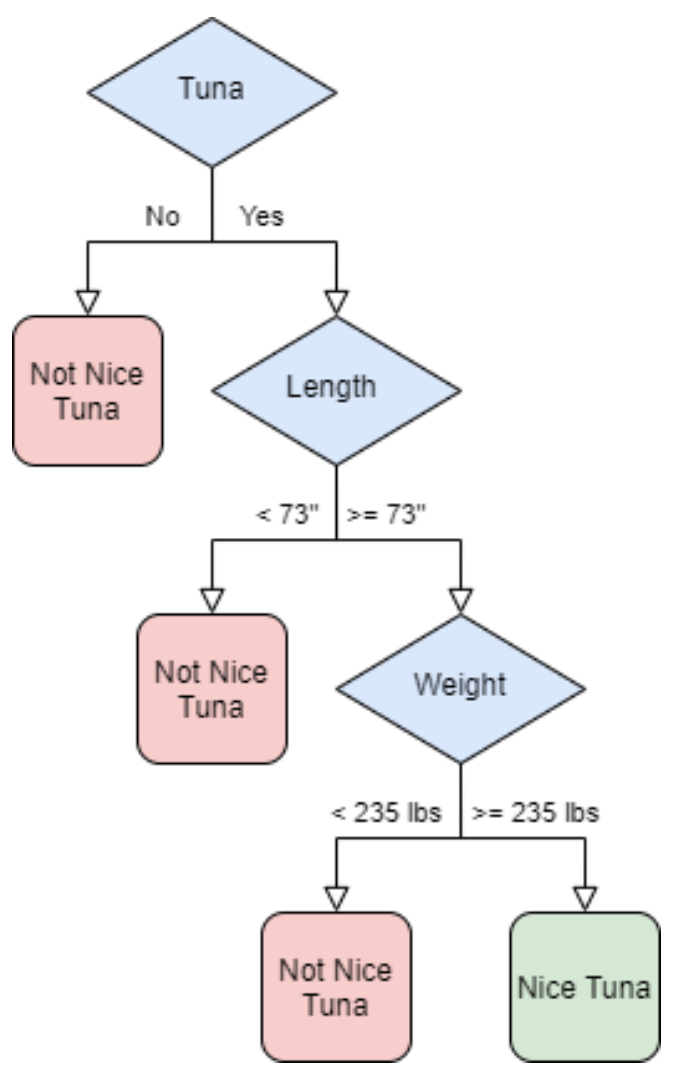

Figure 2.5: An example of a simple decision tree for determining if you have a "nice tuna" or a "not nice tuna".

entropy [50]. Entropy is the fundamental quantity in information theory and was created to quantify the uncertainty of a random variable. Denoted by $H(A)$ or $I\left(p\left(v_{1}\right), \ldots, p\left(v_{n}\right)\right)$, where $p\left(v_{i}\right)$ is the probability of the random variable taking on the value $v_{i}$, entropy is defined according to Equation 2.3 [109].

$$
H(A)=I\left(p\left(v_{1}\right), \ldots, p\left(v_{n}\right)\right)=-\sum_{i=1}^{n} p\left(v_{i}\right) \log _{2} p\left(v_{i}\right)
$$

J48 uses the Info Gain from a feature as its selection criterion. The Info Gain, Gain $(A)$, for a specific feature, $A$, is the expected reduction in entropy that splitting on $A$ will produce, and is defined by Equation 2.4 [109].

$$
I G(A)=I\left(\frac{p}{p+n}, \frac{n}{p+n}\right)-\text { remainder }(A)
$$

In Equation 2.4, the remainder $(A)$ function represents the amount of entropy remaining 
after splitting on attribute $A$. The remainder function is defined as

$$
\operatorname{remainder}(A)=\sum_{i=1}^{n} \frac{p_{i}+n_{i}}{p+n} I\left(\frac{p_{i}}{p_{i}+n_{i}}, \frac{n_{i}}{p_{i}+n_{i}}\right),
$$

where $p_{i}$ is the number of positive class instances for value $v_{i}$ and $n_{i}$ is the number of negative class instances. Info Gain, then, is the difference between the total information content and the amount of information left after splitting on a specific attribute. J48 calculates the Info Gain for each attribute, then splits on the one with the highest value.

Another common splitting criterion for decision trees is the Gini gain, which is a weighted sum of the Gini Impurity for each branch of a decision. Gini Impurity, defined in Equation 2.6, measures the likelihood of incorrectly classifying a new instance if that instance were randomly classified according only to the distribution of class labels in the training data [16]. Gini gain is often chosen in decision tree implementations over Info Gain because it is more computationally lightweight.

$$
G I(A)=\sum_{i=1}^{n} p\left(v_{i}\right)\left(1-p\left(v_{i}\right)\right)
$$

PART is a hybrid learner developed using ideas from both decision tree and rule learners [45]. PART adopts the separate-and-conquer strategy of building sets of rules, but differs in the way individual rules are created. To make each rule, rather than incrementally adding antecedents one at a time, PART builds a pruned decision tree for the current set of instances and makes a rule from the leaf with the greatest coverage, discarding the rest. PART takes its name from this method of generating PARTial trees to create rules, and gains simplicity while saving time by removing the global optimization step.

A RandomForest is an ensemble tree learner, consisting of multiple decision trees [18]. RandomForest uses the technique of bagging to create an ensemble of decision trees to classify instances. Bagging, a contraction of bootstrap-aggregating, is generating multiple versions of a predictor and combining them in some way into an aggregated predictor [17]. In a RandomForest, each tree in the ensemble uses a random vector of attributes from the training set to make the splitting decisions at each node. In a RandomForest, each attribute vector in the set of random vectors is independent and identically distributed. To classify 
an unknown instance, the instance is inputted to each tree in the forest and each tree votes on the class of the instance. The instance is then assigned the class with the most votes. Advantages of RandomForests are their applicability to a wide range of problems, they have few parameters to tune (a computationally expensive process for other algorithms), they achieve high accuracy, are good with small sample sizes or high-dimensional feature spaces, and are easily parallelizable [11]. The latter point is especially inviting, as it has been noted that as number of trees in a RandomForest increases, variance decreases without overfitting. However, increasing the number of trees linearly increases the computational cost. RandomForests are well suited for astronomical searches for their reported accuracy, efficiency in handling large data sets, and robustness with respect to noise.

Boosting, like bagging, is another popular way to improve accuracy through ensemblebased training methods [112]. The goal of boosting is to train new classifiers with instances that have been misclassified by other classifiers. For example, AdaBoost, short for "adaptive boosting", is an ensemble learner which uses several other instances of learning algorithms (such as C4.5 learning trees) in conjunction to achieve better results. In effect, AdaBoost trains multiple weak learners (called "decision stumps", or trees with only one split consisting of one parent and two child nodes) which train on the error sets of the previous learners. Then the ensemble votes on unseen instances. Gradient boosting is another popular method of boosting weaker learners into stronger learners [47]. Instead of weighting hard-to-classify data points (like Adaboost), a gradient boosting algorithm uses a loss function to indicate the goodness of a model's fit to the data. The ability to have customized loss functions specific to the real-world data being analyzed are a key attraction to gradient boosting algorithms.

\subsubsection{Semi-Supervised Machine Learning}

Semi-supervised learning is a type of machine learning that falls in between unsupervised learning, where no class labels are known in the training data, and supervised learning, where all class labels are known in the training data [138]. There are two different broad categories of semi-supervised learning applications: 1) semi-supervised classification, which uses labeled and unlabeled data to train a classifier that is comparable or better than one 
trained on fully labeled data, and 2) constrained clustering, which performs unsupervised clustering with some additional information, such as constraints on when instances must be linked or cannot be linked. For applications in pulsar searching, we propose the use of semi-supervised classification.

Semi-supervised learning is made possible by assumptions about the link between the distribution of unlabeled data and the target label. A few labeled instances are considered to be samples from separate distributions, and unlabeled instances are assumed to inform us about the distribution from which the labeled instances were sampled. The goal of semisupervised learning for classification can either be inductive or transductive, depending on which labels are predicted. Inductive semi-supervised learning predicts labels for unlabeled future test data, and transductive semi-supervised learning predicts the labels for unlabeled training data. The performance of semi-supervised learning algorithms depends on the validity of their assumptions, Furthermore, the assumptions made define the procedures for five different types of semi-supervised learning algorithms: generative, co-training, multiview learning, graph-based, and self-training [101]. Next, we briefly describe each type of semi-supervised learning algorithm.

Generative models assume a model is a recognizable mixture of distributions and that the mixture distribution can be determined from only a few labeled instances combined with a large sample of unlabeled instances.

Co-training models assume that there are two (or more) views of the data (expressible through different feature sets) that provide complementary information about the instances, that each view is enough to make good classifications, and that each view is conditionally independent given the class label. Co-training algorithms create separate classifiers which are trained on labeled data from each view. The highest confidence predictions of one classifier are used to enhance the training set of the other, and vice versa in an iterative manner with the goal of agreement between the two classifiers.

Multiview learning assumes a set of hypotheses exist that agree with each other and that there are few such sets with small empirical risk. Multiview learning models either use separate feature subsets, like co-training models, or use $k$ separate learners in an ensemble. Either way, each learner tries to agree with the other learners and minimize the value of its 
regularized risk. The regularized risk over all learners is the sum of supervised individual risks combined with the semi-supervised regularizer, which measures the disagreement of the learners on unlabeled instances.

Graph-based models assume labels are "smooth" with respect to the graph, and vary slowly, meaning different labels are "far apart" from each other. They operate by defining a graph from labeled and unlabeled training data where the instances are vertices and the (usually undirected) edges represent the similarity between two vertices. The weight of an edge is higher for more "similar" vertices. To quantify similarity between vertices, the edge weight could be calculated according to Euclidean distance in a fully connected graph, or a Gaussian kernel, or a radial base function kernel equation. K nearest neighbor (k-NN) graphs only have connections between neighbors, and thus are not fully connected. $\epsilon$-NN graphs have an edge only if the distance between two vertices is less than some value $\epsilon$.

Self-training classifiers are also called self-teaching or bootstrapping algorithms. They first train on a labeled subset of the training data, then classify the unlabeled data. The highest confidence predictions are added into the labeled training set and used to retrain the classifier, iteratively.

\subsubsection{Metrics for Evaluation of Classification Performance}

To evaluate the effectiveness of our classifiers, we used several performance metrics calculated from confusion matrices [124]. A confusion matrix is a summary table of a classifier's performance on a given test set. In the confusion matrix for binary classification shown in Figure 2.6, the predicted values are represented by the rows non-pulsars and pulsars. The actual values are represented by the columns non-pulsars and pulsars. The result of any classifications then reside in one of the following four boxe:5.

- True Negatives (TN) - represent the number of candidates that were non-pulsars and were correctly classified as non-pulsars,

- False Negatives (FN) - represent the number of candidates that were pulsars, but were

\footnotetext{
${ }^{5} \mathrm{~A}$ confusion matrix can only be computed on a fully labeled data set. If unlabeled instances exist in a data set, they cannot be placed within the confusion matrix and other criteria must be used for evaluation.
} 
incorrectly classified as non-pulsars,

- False Positives (FP) - represent the number of candidates that were non-pulsars, but were incorrectly classified as pulsars, and

- True Positives (TP) - represent the number of candidates that were pulsars and were correctly classified as pulsars.

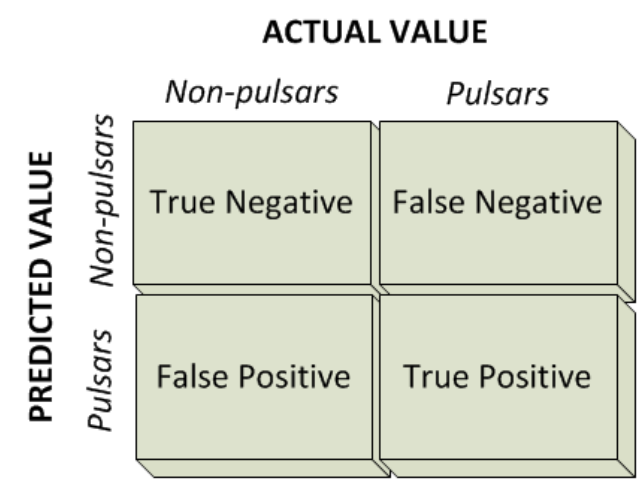

Figure 2.6: Confusion matrix for pulsar classification. The rows represent the predicted class value of the model, and the columns represent the actual values.

For pulsar classification, we are most concerned with the number of true positives and false negatives. Recall is a performance measure that quantifies the ability of the classifier to correctly classify the positive training instances:

$$
\text { Recall }=\frac{T P}{T P+F N}
$$

A perfect recall, or true positive rate (TPR) of 1 , indicates that all positively labeled instances are properly classified.

The false negative rate (FNR) is the complement of the TPR, and represents the conditional probability of mis-classifying real pulsars as non-pulsars, which is very undesirable. It is defined as:

$$
F N R=\frac{F N}{F N+T P}
$$

The precision describes what fraction of the positive classifications are relevant, and is defined as:

$$
\text { Precision }=\frac{T P}{T P+F P}
$$


A perfect precision of 1 means that every instance predicted to be positive was actually a positive instance.

The false positive rate of a classifier describes how often the classifier 'cried wolf', or falsely labeled a negative instance. It is defined as:

$$
F P R=\frac{F P}{T N+F P}
$$

A classifier with a high false positive rate will result in wasted effort to manually inspect non-pulsar candidates, but is more desirable than a high FNR.

For our experiments, we also report the harmonic mean between the recall and precision, commonly known as the F-Measure (F-M) or F1 Score, which is defined by:

$$
\mathrm{F}-\mathrm{M}=2 * \frac{\text { Precision } * \text { Recall }}{\text { Precision }+ \text { Recall }}
$$

The F-Measure has a high value if both the recall and precision are high. This makes it particularly suitable for evaluating the effectiveness of an automated pulsar classifier because it characterizes the ability of a classifier both to not miss pulsars, and to produce fewer false positives that require manual inspection. A perfect learner would have a value of 1 for its F-Measure.

Finally, the F-4 Score is another modification of the F-Measure useful in certain domains where recall is considered more important than precision. The F Score can be generally defined as:

$$
F_{\beta}=\left(1+\beta^{2}\right) * \frac{\text { Precision } * \text { Recall }}{\left(\beta^{2} * \text { Precision }\right)+\text { Recall }},
$$

where $\beta$ is a multiplier for how much more important the Recall is than the Precision. For the F-4 score, $\beta$ is chosen to be four.

\subsubsection{Feature Selection}

In machine learning, feature selection is the process of removing irrelevant features from a data set. Feature selection methods can be either filters or wrappers. Filters are feature selection methods independent of the machine learning algorithms. They rely on statistical or heuristic selection criteria, like correlation or entropy measures, to choose the best features 
[59]. On the other hand, wrappers use the results of machine learning algorithms to perform feature selection. They greedily search the feature space for different combinations of features and evaluate the effectiveness of subsets by the classification performance of a given algorithm [70. Either way, a subset of features is chosen that should increase speed and efficiency of classification by lowering the computational cost and memory usage of the classifier.

One of the simplest feature selection techniques is based off of the concept of information gain (IG). An IG feature selector ranks attributes by calculating their information gain with respect to the class (see Equation 2.4 in Section 2.2.2). A common criticism of IG feature selection, however, is an intrinsic bias in favor of features with a broader range of values [52]. The next two techniques attempt to overcome this bias through some form of normalization of IG.

Gain ratio (GR) feature selectors rank attributes by normalizing the information gain of an attribute, $I G(A)$, by its intrinsic value, $I V(A)$ 64]. The intrinsic value of an attribute represents how much information is needed to place an instance in a branch after splitting on the attribute. For a set of examples, $S$, split by an attribute into $i$ branches each of size $S_{i}$, the intrinsic value of $A$ is given by

$$
I V(A)=-\sum_{i=1}^{n} \frac{\left|S_{i}\right|}{|S|} \log _{2}\left(\frac{\left|S_{i}\right|}{|S|}\right) .
$$

The gain ratio is then given by

$$
G R(A)=\frac{I G(A)}{I V(A)}
$$

The symmetric uncertainty $(\mathrm{SU})$ between two attributes is a normalization of $I G(A)$ that always falls between 0 and 1 . SU depends on the joint entropy, $H(A \mid B)$, of an attribute $A$ with values $a_{i}$ (see Equation 2.3) and an attribute $B$ with values $b_{j}$. Joint entropy is calculated from the joint probabilities of all combinations of values $a_{i}$ and $b_{j}$, and is given by

$$
H(A \mid B)=-\sum_{i=1}^{n} p\left(b_{i}\right) \sum_{j=1}^{n} p\left(a_{i} \mid b_{j}\right) \log _{2}\left(a_{i} \mid b_{j}\right),
$$

where $p\left(a_{i} \mid b_{j}\right)$ is the conditional probability of value $a_{i}$ given value $b_{j}$. A SU feature selector ranks each attribute, $A$, by calculating its $\mathrm{SU}$ with respect to the class, $C$, according to 
Equation 2.16 [124].

$$
S U(A)=2 \frac{H(A)+H(C)-H(A \mid C)}{H(A)+H(C)}
$$

The feature selection method using linear correlation (Cor) ranks attributes by calculating the Pearson coefficient of correlation, $r$, between each attribute, $A$, with values $a_{i}$ and the class, $C$, with values $c_{i}$ [69]. Pearson's $r$ for $n$ samples is given by

$$
r=\frac{\sum_{i=1}^{n}\left(a_{i}-\bar{a}\right)\left(c_{i}-\bar{c}\right)}{\sqrt{\sum_{i=1}^{n}\left(a_{i}-\bar{a}\right)^{2}} \sqrt{\left.\sum_{i=1}^{n}\left(c_{i}-\bar{c}\right)\right)^{2}}}
$$

where $\bar{x}$ is the sample mean for the values of attribute $X$.

$1 R$, short for '1-rule', is an inexpensive rule learner that generates one simple rule for each value of each attribute. The rule assigns each attribute-value pair the class value that occurs most often for that pair. The classifier then calculates error rates over the whole set for each rule and retains the rules with the lowest rates [124]. This simple algorithm can be converted into a feature selection technique by summing the error rates for all rules created for each attribute, and then ranking the attributes accordingly. 


\section{Chapter 3}

\section{Related Work}

This chapter presents an extensive survey of the literature pertaining to our work. The first section reviews classification studies performed using periodicity searches, the second section presents pulsar searches within single pulse search output, the third section compares and contrasts several existing peak identification algorithms with our proposed technique, RAPID, the fourth section reviews related work on machine learning with imbalanced data, the fifth section presents work from high performance computing in astronomy, and the sixth and final section reviews related work on semi-supervised learning.

\subsection{Related Work on Periodicity Searches}

Classification techniques in the literature for periodicity search candidates include both automated heuristic approaches ${ }^{1}$ and machine learning approaches. As our focus is on machine learning, we only provide reviews of papers that use machine learning techniques [41, 17, 137, 90, 84, 118, 10, 132, 49, 21, 128, 128]. The fact that these papers were all published in the last ten years indicates that intelligent algorithms are the new standard for pulsar classification.

In [41, the authors used artificial neural networks (ANNs) to automate pulsar detection in the PMPS. They used a set of twelve features, including the pulse profile SNR, pulse profile width, and $\chi^{2}$ values of fits to theoretically optimal curves. Their training set consisted of

\footnotetext{
${ }^{1}$ See [4, 67, 75].
} 
259 examples of known pulsars combined with 1,625 non-pulsar examples of noise or RFI. Their model led to the discovery of one pulsar.

ANNs were also used in [7] to classify candidates. The authors expanded the input features from [67] and [41] to include $\chi^{2}$ values for fits of the pulse profile to Gaussians and sinusoids, and profile histogram tests. Their resulting ANN was able to detect $85 \%$ of pulsars in controlled tests with data from the HTRU survey. It was further found that the ANN's classifications depended on the training data used, leading them to recommend a representative sample of pulsars to increase the accuracy of the learner. This work resulted in the discovery of 75 pulsars.

The Pulsar Image-based Classification System (PICS) was developed as an artificial intelligence program to identify pulsars using image recognition algorithms [137]. PICS consisted of two layers and was designed to emulate a human expert's visual identification process. The first layer is a group of trained image learners (ANNs, convolutional neural networks $(\mathrm{CNNs})$, and support vector machines (SVMs)) which examine and score candidate subplots. These scores are combined using a logistic regression model to minimize classification errors in the training data. The PICS AI system was tested on the Green Bank North Celestial Cap pulsar survey and is currently integrated with the PALFA survey, where it has discovered six pulsars.

Classification results from a pulsar ranking system called Straight-forward Pulsar Identification using Neural Networks (SPINN) was presented in [90]. SPINN uses a customized ANN trained on 1,196 observations of pulsars from the HTRU all-sky pulsar survey combined with 90,000 randomly selected negative observations. They were able to correctly classify all known observations of pulsars in the HTRU data while reducing the number of candidates requiring manual inspection by several orders of magnitude. This system was responsible for the discovery of four pulsars.

Candidate selection for LOTAAS was performed by using a purpose-built tree-based machine learning classifier, the Gaussian Hellinger Very Fast Decision Tree (GH-VFDT) [84]. In general, VFDTs are used when training data cannot fit into main memory and needs to be faster than sequential disk scanning of data sets from a hard disk drive, such as in very fast incoming data streams. The authors also used a new set of features for describing 
candidates that were selected to maximize the separation between candidates arising from noise and those of probable astrophysical origin, and be as survey-independent as possible. They performed a comparison of GH-VFDT to four classifiers implemented by Weka (J48, MPN, NB, and SMO) using data from LOTAAS and both HTRU surveys achieving relatively high recalls $(0.928,0.829,0.789)$ and low FPRs $(0.005,0.008,0.001)$.

In [118], the authors implemented several improvements to the machine learning classifier used by LOTAAS. The improvements consisted of: introducing a third RFI class and new features, train on pulsars missed by other algorithms, and using an ensemble decision tree classifier. They achieved improvements on recall (0.987) with reduced FPRs (0.005).

In [10], the authors evaluated four unaltered algorithms: an ANN (MPN), and three boosting algorithms (Adaboost, Gradient Boosting Classifier, XGBoost), all implemented in the Python package SciKits ${ }^{2}$. They performed supervised machine learning experiments by extracting six features from HTRU candidates and treating their training data sets using SMOTE. They repeated their experiments 20 times using 5 -fold cross validation and achieved extremely high recalls for all algorithms (>0.995).

A small, fully labeled subset of the HTRU candidates (1,196 positive examples and 89,996 negative examples) were used to test a CNN in [132]. The authors used the CNN to perform "feature engineering", i.e., allowing the algorithm to perform feature selection directly from the data. To accomplish this, they normalized the data and used an ANN to select features by performing multiple classification trials using one feature only, ranking the classifiers by accuracy, and selecting the highest ranking features. They trained their final model on data balanced by oversampling using 10-fold cross validation and reported a recall of 0.94 and F-Measure of 0.92 . We suspect that the model may suffer from overfitting, due to our own results with oversampled training sets presented in Section 5.4.2.

Another attempted image recognition solution with periodicity plots can be found in [49]. The authors recognized that Deep CNNs (DCNN) have problems trying to classify pulsars due to limited training samples. To solve these problems, they proposed Deep Convolutional Generative Adversarial Networks (DCGAN) with SVMs. DCGAN dealt with class imbalance by generating positive samples by treating the problem as two player competitive game

\footnotetext{
${ }^{2}$ See https://www.scipy.org/scikits.html.
} 
between a discriminator, and a generator. The discriminator tried to predict the correct label of an input sample (real or generated), while the generator tried to create samples which cannot be correctly classified by the discriminator. They tested DCGAN on a fully labeled subset of the PMPS data (2,000 positive examples, 2,000 negative examples, 20,000 RFI examples) and the same subset of HTRU data as [132. They initially train the classifier with an equal number of pulsar non-pulsar samples. After the initial training, the generator created samples similar to actual samples and the discriminator predicted the probability that the generated samples came from the real data set. The activation values of the discriminator's max-pooled middle layer were used as "deep features" for classification by a final SVM. Their DCGAN, SVM combination achieved great results, with F-Measure/Recall values for the HTRU data (0.963/0.96) and the PMPS data (0.889/0.895).

A recent work compared logistic regression, RandomForest, and Knn on the Southern HTRU survey data [21. Their results showed that the RandomForest classifier using eight features to characterize a periodicity search candidate exhibited the best F-Measure (0.886).

Finally, in the most recent work reviewed, the authors proposed a non-parametric PseudoNearest Centroid Neighbour Classification (PNCN) algorithm for periodicity candidate classification [128]. They quantified the harmfulness of class imbalance problem by using "scatter matrices" to determine within-class scatter and between-class scatter and used the results to describe the degree of "overlapping" of the class instances. The results were then used to inform the training of subsequent algorithms. If the measured class imbalance was considered harmful, then they would use an imbalance treatment to counteract it. They claimed that their algorithm boosted performance over regular Knn because Knn suffers from sensitivity to sparse, imbalanced, noisy data. They tested PNCN on LOTAAS and Northern HTRU data using eight features and 5-fold cross validation. The experiments resulted in high F-Measures for the LOTAAS data (0.874) and the HTRU data (0.874).

\subsection{Related Work on Single Pulse Searches}

The first theoretical framework for performing single pulse searches to detect fast radio transient signals was presented in [29]. The authors' proposed automated detection approach 
first removed the baseline from the dedispersed data, then utilized an N-sample boxcar filter to detect significant events, which they plotted for manual inspection. They also explored a range of widths by adding a number of adjacent samples, smoothing the data, and then searching for single bright pulses. To avoid bias towards very strong individual pulses, they removed the brightest pulses in the first pass, then searched again. They recorded the DM, the arrival time of the pulse relative to the start of the observation, and the SNR for each pulse subsequently detected. The methodology presented by this work was adopted, in one form or another, in each of the following papers.

In [32], the authors presented results from radio transient searches using data from the PALFA survey. They performed matched filtering similar to [29], but with a more sophisticated RFI excision scheme. Their search was customized to remove two types of RFI: RFI from radar unique to Arecibo (from the San Juan airport), and RFI simultaneously detected in several beams. They also used a friends-of-friends clustering algorithm, which formed the initial clusters by searching for events above a given SNR threshold, then added to the clusters by including adjacent samples above a given threshold. The brightest sample of a cluster was recorded as the cluster amplitude and the number of samples as the width. This search was less sensitive to weak, narrow pulses but resulted in a significant reduction of RFI events and resulted in seven pulsar discoveries.

A re-analysis of the PMPS data which discovered ten RRATs was performed in [65], suggesting that the population of transient radio-emitting neutron stars may be larger than initial predictions. The authors searched for bright single pulses using matched filtering, as did [29]. To eliminate RFI, they used the "zero-DM filter", developed by [40], and also removed multi-beam events from consideration, as in [32]. They produced diagnostic plots for manual inspection and classification.

The authors in [20] presented the initial results for an examination of the HTRU survey using similar search techniques. They stored parameter values in a database, which was then queried to see if events have more than two members and peak at a DM over $1.5 \mathrm{pc} \mathrm{cm}^{-3}$. If so, summary plots were created of the events for manual assessment. Their efforts resulted in eleven discoveries of sparsely emitting neutron stars.

Using an iterative process to extract individual pulses, the authors in [108] detected sev- 
eral single pulse events, some of which were repetitive, in a search of the Andromeda Galaxy and its satellites with the Westerbork Synthesis Radio Telescope. The authors searched for single events with SNR i 7.0 or multiple, fainter pulses repeated for a single DM.

A search for RRATs in data from the GBT350Drift data was presented in 62. After applying similar filtering techniques, the authors grouped the data according to their relative positions in the DM vs time space and divided each group into five bins. The neighboring bins were then checked to see if the maximum SNR in each one was monotonically decreasing and created diagnostic plots for manual inspection. This work resulted in the discovery of eighteen RRATs.

The papers presented above all include automated search techniques using heuristics, e.g. sifting candidates by known SNR or DM thresholds. Our work differs from the literature by not relying on heuristic thresholds to identify peaks, and by using supervised and unsupervised machine learning to develop intelligent classifiers.

\subsection{Related Work on Peak Identification}

Peak or trough identification is a common problem in many fields that require signal processing. Many different techniques have been proposed to solve this problem, ranging from general solutions to solutions highly specific to particular fields. In this section, we briefly discuss several existing peak identification approaches and describe why a new technique was required to identify peaks in single pulse search candidates.

A common approach for identifying peaks in time series data is to detect local maxima by noting sign changes in the slopes between a single point and its immediate neighbors. A major problem for this, and all peak detection algorithms, is their sensitivity to noise. Another popular solution is to first smooth the data with some sort of filter and then fit a given function to it [96].

In mass spectroscopy, peaks have specific shapes. Taking advantage of this fact, a pattern matching algorithm was developed in [39] using continuous wavelet transforms (CWTs). The basic shape of the peak was assigned to the wavelet function, which was in turn used to compute an array of CWT coefficients according to multiple scales. Peaks were then 
identified as "ridges" formed in the wavelet space.

In [54], the authors proposed an algorithm to detect peaks and troughs based on momentum. The "momentum" was found by taking the product of the value of a data point and the rate of change at that point. A theoretical ball was then "rolled" from a known peak. As the ball descended the peak, its momentum increased and then decreased as it climbed another peak. When the momentum reached zero, the ball was considered to have come to rest and that point was declared a new peak. Momentum changes were also affected by Newton-esque laws of motion, such as an analogue to friction.

In astrophysics, several burst detection algorithms have been proposed to identify gammaray bursts (GRBs). The Li-Fenimore algorithm (LFA) operated by binning the data and then labeling as a candidate peak each bin that had more counts than its immediate neighbors [77. A search was then conducted for each candidate peak to determine if the counts for non-immediate neighbors (more than one bin away) continued to diminish according to a given formula.

The authors in [139] proposed a burst detection algorithm to identify GRBs in real time. Their algorithm relied on wavelets by introducing a new data structure called the shifted wavelet tree (SWT), which was used to organize wavelet coefficients and additional information about the window by resolutions and time scales. The elastic window was created by automatically scanning different time resolutions and sizes and determining the window size accordingly.

In [48, the authors developed MEPSA, an algorithm similar to LFA that also used binning and the counts of signals in each bin to detect GRBs. MEPSA utilized 39 userdefined patterns to help peak identification. For each bin, the adjacent bins were searched to see if they fit any of the different patterns. MEPSA was more reliable than LFA, but came with an added overhead of 39 separate pattern comparisons for each possible peak.

We created RAPID because machine learning for DPGs and single pulses has several requirements, and none of the algorithms listed above satisfied all of these requirements. First, identifying the peak alone is not sufficient; pertinent features must be extracted which include the shape and number of pulses in the range of the DPG, from beginning, to peak, to end. This necessitates that any detection routine be capable of identifying more than just 
local maxima in the data. Second, focusing on single values and ignoring local trends could overlook peaks in the data. Third, not all pulsar candidates have a set width or height and are often very faint or buried in noise. Finally, due to the large quantity of radio data to be examined, the algorithm used for detecting pulsar candidates must be simple and efficient, ideally making only one pass through the data.

\subsection{Related Work on Imbalanced Data}

Imbalanced data sets have a tendency to cause problems with supervised machine learning algorithms. The works presented in this section attempt to solve those problems with various techniques of restoring the balance to imbalanced data sets. Their results weighed in on our decisions concerning handling the imbalance inherent in our data.

The authors in [116] introduced three cost-sensitive, boosting algorithms into the AdaBoost framework to place higher importance on identification of selected underrepresented classes. They provide some specific solutions for dealing with unbalanced data, including fine-tuning parameters for learners, applying cost-sensitive weights to learners by building in penalties for misclassification, and randomly oversampling the minority class for training. They experimented with several of these techniques and were able to achieve very high recall values, but with low precision.

For training decision tree learners on unbalanced data, [26] suggested that common splitting criteria for making decisions in tree learners, could be replaced with a criterion less sensitive to skewed class distributions. They offered the "Hellinger distance", a measure of divergence between class distributions, as a skew-insensitive alternative for finding decision tree splits. They compared splitting decision trees using the Hellinger distance (HDDT) to the standard tree algorithms C4.5, and classification and regression trees (CART), [16], as well as to "DKM trees" [36]. The results showed HDDT and DKM produced superior decision trees when trained on skewed data, however, they also demonstrated that sampling improved results for C4.5 and CART, but not DKM or HDDDT.

Finally, [95] proposed evolutionary, online rule learners that genetically evolved a single set of rules called Learning Classifier Systems (LCS). The first LCS presented, XCS, is unsu- 
pervised and used customized quality estimators, an iterative performance component, and a genetic search component, i.e., offspring rules were introduced based on crossover between parents and random mutations. They also described UCS, which is a supervised learning alteration to XCS that made use of evolutionary pressure to develop complete, consistent, and minimally representative rules. They performed experimentation to compare these algorithms to decision trees (C4.5), support vector machines (SVM), and instance-based learners (IBk) which showed that LCSs performed well when their parameters were properly tuned. The experiments were repeated using the resampling techniques of oversampling, undersampling, SMOTE (see Section 4.4.2), and CSMOTE (a clustered implementation of SMOTE). The results showed that resampling techniques generally improved results for all learners, and specifically using oversampling and SMOTE with C4.5 trees provided better classifiers than other techniques.

\subsection{Related Work on High Performance Computing}

Many recent works have applied data analytics to classification problems from radio astronomy (see [34, 97] for more detail). While execution performance for scientific applications with Big Data has been considered in other areas (see [2, 3, 30, 102]), most of the literature from radio astronomy, including our previous work, is focused on classification performance with no consideration of execution performance. In other areas of astronomy, however, several recent papers have attempted high performance computing solutions.

A set of Python extensions for exploratory data analysis in astronomy called Pydron was presented in [91]. Recognizing that scalable computing solutions to science problems are complex and have a steep learning curve, the purpose of Pydron was to automatically parallelize code chunks for cloud computing based on a data flow graph, making scalability accessible to scientists. In a data reduction experiment involving optical image source detection and measurement, Pydron reduced execution time from more than four hours to under three minutes.

The authors in [55] approached the problem of requiring experts to review candidates by proposing two principal components: 1) a processing pipeline for extracting and cataloging 
the metadata for candidate events, and 2) an interactive, Web-based data portal to facilitate collaborative review and classification of these event candidates. Their solution relied on Apache Object-Oriented Data Technology for data integration and used Apache Solr for rapid querying, subsetting, and searching of the data sets.

In [46], the authors treated the simulation of small solar system bodies as an N-body problem using fine-grained multithreading. Since many asteroids and comets are clumps of small particles, they proposed a fully parallel, shared-memory algorithm for dense selfgravitating agglomerates. Their algorithm was a combination of related algorithms and substituted a smooth fitting function for the exact value of gravitational potential, used hierarchical grouping to speedup computation on grid nodes, and divided space into a regular and static grid. To achieve high performance, they performed domain decomposition by dividing the application data into disjoint sets and processing it in parallel, and functional decomposition through the parallel execution of functional units that performed different control tasks. Domain decomposition was accomplished with master-slave communications using shared memory to reduce the communication cost. They evaluated their parallel implementation on multicore machines, with promising results.

A parallel implementation of the $\mathrm{k}$-means clustering algorithm called Para-Heap- $\mathrm{k}$ was presented in [58]. To evaluate their extension of k-means to a parallel architecture, they simulated a galaxy with one million stars in a synthetic star survey and attempted to cluster the bulge, thick disk, and thin disk. To reduce the amount of data processed at each iteration, Para-Heap-k stored cluster centroids and associated points in heaps so that points closer to the centroid are higher in the heap. They also enforced a restraint to keep far away points at the bottom, and only consider leaf nodes when performing calculations. As the heap is updated, data not in the leaves may get pushed down to a leaf.

It appears that the only work to account for execution performance in radio astronomy applications was presented in [134], which used Apache Spark to develop a toolkit called Kira SE to parallelize source extraction for astronomy image processing. Through several experiments processing a 1 TB dataset show that the distributed version of Kira SE significantly outperformed a version coded in $\mathrm{C}$ and that a distributed Apache Spark platform on the Amazon cloud is a competitive alternative to using supercomputers. Our approach differs 
from [134 by being more "white box", i.e., we address specific performance bottlenecks in the process of candidate identification and classification.

\subsection{Related Work on Random Forests}

The RandomForest ensemble tree learning algorithm (briefly described in Section 2.2.2) was first introduced by Breiman in 2001 [18]. Based on our favorable results achieved with RandomForests on radio astronomy data, the culmination of this dissertation proposes a novel, distributed RandomForest algorithm. This section presents a review of RandomForestbased algorithms from the literature in chronological order. For an excellent survey of RandomForests in work published before 2014, see [140, 11].

Enriched RandomForests, proposed in [4], focused on reducing the number of trees whose nodes use non-informative features by using weighted random sampling with weights tilted toward informative features. The algorithm was designed for data with many non-informative features, specifically genetic data and calculated weights for features by scoring each gene via a two-sample t-test, using the q-value. The authors chose the q-value because it provides false discovery rate (FDR)-adjusted measures of significance for the features, over the pvalue, which provides expected false positive rate. The algorithm was tested using eight public genetic databases and had significantly less Out of Bag Error when compared to a standard RandomForest implementation.

The authors in [9] proposed Dynamic Random Forests, which attempted to create trees that are as complementary as possible, instead of the independent trees of the standard implementation. They based their idea on previous work that showed there was always one subset of a RandomForest that significantly outperformed the initial when the number of trees is large (they used 200). The proposed algorithm utilized both bagging and boosting to create complementary trees. The algorithm operated as follows: first, a certain number of instances were randomly sampled with replacement from the training set to create a decision tree (bagging). Second, the training data was weighted according to the previous tree's performance on the out of bag samples, with poorly classified instances receiving heavier weights (boosting). As the process was repeated, each new tree trained on the 
data "missed" (heaviest weights) by most other trees. The reason for this choice was to keep using the two efficient randomization processes, i.e., bagging and Random Feature Selection, of Breiman's RandomForest, while improving prediction accuracy by using the adaptive resampling principle of boosting. The authors evaluated Dynamic RandomForests on twenty data sets and had lower error rates than the standard RandomForest for nineteen of them.

Weighted RandomForests were proposed in [123] for data sets with high dimensionality, such as those found in genetics. These forests incorporate tree-level weights to emphasize more accurate trees in predictions rather than using simple majority voting. While their particular weighting scheme did not show significant improvements over a traditional RandomForest on several real-world genetics problems, many other weighted-voting schemes are possible and may show promise. Our proposed new distributed, semi-supervised algorithm also uses the intuition that selecting trees that perform well may improve ensemble performance.

Alternating Decision Forests, proposed in [113], treated the training of Random Forests as a global loss minimization problem. During training, the losses were minimized via keeping an adaptive weight distribution over the training samples, a form of boosting. Alternating Decision Forests kept a weight distribution for all training instances in each iteration which would be updated, such that "easy" samples are down-weighted and "hard" samples are assigned heavier weights. This allowed the model to emphasize training on misclassified instances (boosting). Evaluation on five common data sets yielded better results than the standard RandomForest.

In [71], the authors proposed a computationally lightweight approximation model based on probability theory for feature selection frequency in RandomForests to control false positive rates. They used their model to determine the splitting threshold between relevant and non-relevant features in a "principled way". They described two general training strategies: 1) randomly choose a new subset of features from the entire feature set at every node, independently from the others (which is the most common strategy), or 2) independently choose a random feature subset for each tree, but use the same subset to train all the nodes within the tree. Evaluation on 100 generated data sets showed that the model could "accurately 
capture the behavior of selection frequency".

The authors in [115] noted that the original RandomForest has two major drawbacks: 1) the heuristic learning rule does not effectively minimize the global training loss, and 2) the model size is usually too large for many real applications. They proposed two techniques to remedy this, global refinement and global pruning. Global refinement jointly relearns the leaf nodes of all trees under a global objective function so that the complementary information between multiple trees is well exploited, enhancing fitting power. Global pruning independently merges leaf nodes of individual trees using global optimization. Their experiments showed the refined model had better performance with a smaller memory footprint.

In addition to Info Gain or Gini Impurity, several other authors proposed alternative methods for the problem of selecting meaningful split points for RandomForests. The authors in [33] proposed a Regularized RandomForest algorithm, which penalized selecting a new feature for splitting when its gain was similar to the features used in previous splits. Reinforcement Learning Trees, proposed in [136], to split on the feature that brings the greatest future improvement in later splits, rather than choosing the one with largest marginal effect from the immediate split.

A parallel distributed implementation of RandomForests for Big Data using Apache Spark was proposed in [25]. This approach incorporated four major improvements: 1) data-parallel optimization of the training set, 2) task-parallel optimization, 3) dimension reduction, and 4) weighted voting. For their data-parallel optimization, they split the data up among the nodes in the cluster by column (features) instead of by row (instances), a strategy they call "vertical partitioning". They also performed data multiplexing, a modification to traditional sampling. Data multiplexing involved making a separate table containing sampling indices for all sample sets, i.e., each row of the table gives the randomly selected indices of instances for one sample of the training data. Then each slave receives the table and a single feature subset (vertical partition from above) and calculates the Gain Ratio of its feature for the instances contained by each of the samples. To achieve task parallel optimization, they parallelized the training process by splitting it into gain-ratio-computing and node-splitting tasks, then scheduling those tasks in parallel. Dimension reduction was performed during training and accomplished by calculating the Gain Ratio for each feature, sorting the features 
in descending order, and keeping the top features along with several additional randomly selected features. Finally, they implemented weighted voting during the prediction phase by weighting the votes of each tree in the forest by its classification accuracy on the testing data $^{3}$. Their results compared to other RandomForest parallel implementations showed comparable classification performance with much better execution performance, including slower-increasing run times as data size increased, speedups of up to 85 times for many nodes on some data sets, constant training set sizes (others increased linearly), and a slow, linear increase in shuffle writes (others were exponential).

The most recent and last of the reviewed works both come from the astronomy domain. Probabilistic RandomForests, proposed in [106], attempted to take into account uncertainties in feature values as well as in assigned classes (i.e., labels) by treating the features and labels as probability distribution functions instead of deterministic values. In a traditional RandomForest, a new instance has a single trajectory through a tree according to the binary criteria in the different nodes. In an ideal Probabilistic RandomForest, an instance would propagate through all branches, with probabilities calculated and stored along the way. This is unrealistic to implement computationally, so the authors proposed an approximate version where an instance is propagated through every branch until its probability drops below a set threshold. This results in the algorithm taking only the high probability branches into account, reducing the runtime. Their results showed that this new algorithm outperformed the traditional RandomForest when noise was injected into data sets.

Finally, in [133], the authors used spectroscopic and photometric data to train modified RandomForest classifiers to detect a rare star type. They addressed the imbalance problem in the data by using cost sensitivity. Their implementation was an alteration of the standard SciKit implementation with costs adjusted according to class representation in the data, meaning that they weighted the importance of instances inversely by the frequency of their class value. This method achieved very promising results on data from the Sloan Digital Sky Survey.

\footnotetext{
${ }^{3}$ Performing weighted voting in this manner, in our opinion, should exhibit a tendency to overfit the testing data. The weights should be calculated on the training, not the testing data.
} 


\subsection{Related Work on Semi-Supervised Learning}

The main focus of our work with semi-supervised learning algorithms focuses on cotraining and self-learning. The difference between co-training and self-learning, as described in Section 2.2.3, occurs during the labeling phase of each iteration. In co-training, one classifier is used to label training data that will be used by another classifier, while in a selftraining scenario a classifier adds the instances it labels into its own training set. This section contains a review of several works from the literature related to our work with co-training and self-training algorithms, followed by a brief section describing works with semi-supervised decision trees and their applications in radio astronomy.

\subsubsection{Co-Training}

The original co-training paper split the problem of web page classification into two views: 1) words on the web page and 2) words on the pages linking to the web page [13]. The authors trained two text-based Naïve Bayes classifiers, one on each view, and then used their predictions on unlabeled data to enlarge the training sets for each other. They randomly selected unlabeled instances for each classifier and only the most confident positive and negative predictions were added to training sets, in a ratio consistent to the class distribution. In tests on 1,051 web pages from four universities with $22 \%$ positive examples, the co-training classifiers had lower error rates compared to Naïve Bayes trained on only twelve labeled instances.

In [93, the authors further examined the co-training algorithm from [13]. In additional experiments, the showed that co-training classifiers which leveraged a natural, independent feature split outperformed ones that did not. Their classifier performed better than an expectation maximization classifier with only six labeled instances. They further showed that when a natural split does not exist in the data, algorithms that manufactured a split outperformed algorithms not using a split at all, however, their results in this area were not very good, with error rates around $30 \%$.

The authors in [80] provided methods of empirically verifying whether a data set violates the two basic assumptions of co-training, i.e., the two views of the data are independent given 
the class and sufficient on their own to perform classification. While these assumptions can sometimes be verified by domain knowledge, they provide empirical methods using 10-fold cross validation of the two views to evaluate sufficiency and independence. They also stated that success will be limited for any standard two-view co-training classifier on most real-world data sets with single views.

Self-Paced Co-Training was proposed in [85] to counter problems the authors saw with the independence and sufficiency assumptions in existing co-training theory. They described self-paced training in general as an evolution of curriculum learning, where a model is learned by gradually including more complex samples during training to increase the entropy of training samples [8]. Self-paced learning embeds curriculum design as a regularization term into the learning objective [72. Their Self-Paced Co-Training classifier worked iteratively by weighting and updating weights which determined the importance of two views in classification. They evaluated their classifier on six text classification data sets with $14-50 \%$ labeled instances and compared against six other semi-supervised learning methods, including self-training and standard co-training. The results showed that Self-Paced Co-Training outperformed all other classifiers using accuracy as the only metric (0.8644 Accuracy, 0.02 better than standard co-training).

Co-training for INductive Semi-supervised multi-label learning (COINs), proposed in [131], was an attempt to make inductive semi-supervised learning classifiers for multi-label learning. Multi-label learning is when one instance can belong to multiple classes, i.e., have multiple labels. In each co-training round, COINS attempted to maximize the diversity between the induced classifiers and then iteratively communicated the pairwise ranking predicitons of either classifier on unlabeled instances for model refinement. COINs communicates only the single predicted, most relevant label and the single predicted, most irrelevant label between two classifiers. In an experimental evaluation on ten benchmark multi-label data sets using, COINs performed favorably.

The authors in [57] proposed a semi-supervised classifier based on improved co-training, which effectively utilized labeled data and unlabeled data to improve classification performance when applied to image recognition in polarimetric synthetic aperture radar, where supervised and unsupervised classification are prevalent. They further introduced a novel 
sample selection scheme is designed, which selected high confidence samples to enlarge the training set and improve the co-training process in two stages. The first stage, pre-selection, narrowed the data to only pick from highly reliable subsets of the unlabeled data. They made two assumptions when selecting samples: 1) that the unlabeled data had local consistency (the same areas were labeled the same way), and 2) a "similarity hypothesis", stating that regions with high similarity should have the same label. For the second stage, sample selection during co-training, they introduced the "worst case classification rate", an indirect analysis strategy to analyze the independence between two views by performing feature extraction on coherency matrices of polarimetric features. They evaluated independence of the views with Spearman's rho correlation coefficient, the double-fault measure (percent of cases misclassified by both classifiers), a disagreement measure (ratio of observations where one classifier is correct and the other is incorrect), and McNemar's Statistic. They also proposed a new post-processing method to reduce "speckle noise" inherent to their data domain and improve the consistency of classification results. When testing for sufficiency and independence of the two views, they recommended: classification accuracy greater than 0.5, disagreement greater than 0.3 , and correlation of predictions less than 0.5.

The authors in [126] noted a problem with traditional co-training: "selection of samples in existing co-training methods is based on a predetermined policy, which ignores the sampling bias between the unlabeled and the labeled subsets, and fails to explore the data space." Basically, they claimed that randomly sampling the unlabeled data results in a high sampling bias shift toward the unlabeled data and selecting only high-confidence examples will contribute little for boundary instance discrimination. To overcome this problem, they introduced Reinforced Co-Training, which applied Q-learning to learn an inclusion policy from a smaller labeled subset of the data, which is then applied to the co-training. They used a Q-agent (a form of multi-layer perceptron network) to select high-quality, unlabeled subsets for training by reinforcing the agent with performance-driven rewards and then organized the unlabeled data set by partitioning according to Jaccard similarity. This approach was designed for and applied to Natural Language Processing, where the two views are the headlines and content of articles. They evaluated this method on two data sets against eight other semi-supervised learning implementations, and the results showed that their Re- 
inforced Co-Training classifier performed the best. However, they noted that their results indicated a serious gap between semi-supervised methods and supervised methods.

Multi-label co-training (as in [131]) was revisited by the authors in [129]. They attempted to address two issues: 1) class imbalance in multi-label data sets by computing label correlations, and 2) communicating more than one label and its confidence between cooperating classifiers. They performed evaluation of their method using four data sets with $10 \%$ of the training set randomly selected to include labels, repeated ten times.

\subsubsection{Self-Training Random Forests}

Co-Forest, proposed in [78], blurred the line between self-training and co-training by applying co-training to RandomForests. The authors noted that confidence estimation for newly labeled instances is a crucial step for co-training and offered a new algorithm that did not depend on predetermining two sufficient and independent views of the data. The three main differences between their algorithm and standard co-training were: 1) they used ensembles of trees as their different classifiers, 2) prediction confidence estimation was determined by a "concomitant ensemble", which consists of the classifiers NOT used to assign the prediction in question, and 3) they attempted to reduce the negative effect of large amounts of artificial labels by weighting their instances based on the predictive confidence calculated by the concomitant ensemble. They validated this approach on nine common data sets against RandomTree, RandomForest, SVM and AdaBoost classifiers by treating 80\%, 60\%, 40\%, and $20 \%$ of the data as unlabeled. Overall, the $\mathrm{Co}=$-Forest showed an average improvement in error rates of $11.5 \%-14.2 \%$, which increased as more of the data was unlabeled.

Semi-Supervised RandomForests were proposed in [76]. The authors noted that RandomForests are good candidates for semi-supervised learning due to their ease of parallelization, speed, ability to handle multiclass problems, and insensitivity to label noise. Individual trees in the forest, however, should be as diverse as possible, which can be an obstacle for semi-supervised learning, since the trees must come to an agreement on unlabeled data for transductive learning. The problem with making semi-supervised learning RandomForests is that RandomForests do not benefit from the traditional, regularized loss functions com- 
mon with semi-supervised learning trees because the regularization destroys the diversity. Their proposed Semi-Supervised RandomForests attempted to maximize the margin of the RandomForest over the unlabeled samples, treating the scoring and training of the RandomForest as an optimization problem. They used Deterministic Annealing (which has randomness built in to maintain diversity among the trees) to maximize the multiclass margin for the unlabeled data. To evaluate performance, they randomly partitioned fully labeled data sets by ten repetitions of treating $5 \%$ as labeled and $95 \%$ as unlabeled and reporting average classification accuracy. Their algorithm performed slightly better than four others in comparison, but was slower in serial form. However, when the code was parallelized to run on a GPU, it was three times faster than its serial form.

minSemi-RandomForest was proposed for classification in the educational outcomes domain in 22. The classifier used three trees and operated according to a simple, self-training algorithm. In iterations, it trained a RandomForest with three trees on the labeled set, keeping the classifier if it had a better error rate. Then, if the unlabeled set was not empty, for each unlabeled instance the classifier would redict a label, score the prediction, and add it to the labeled set if the confidence was high enough. Experimental results showed improved accuracy over RandomForest, Self-Training C4.5, Tri-training, and Co-forest.

\subsubsection{Semi-Supervised Algorithms in Astronomy}

Semi-supervised learning is a machine learning technique that aims to make accurate predictions on unseen data by learning patterns from partially labeled training sets. Since labeling instances for pulsar candidate classification is expensive, semi-supervised learning is a promising direction for radio astronomy. The first three papers, [37, 79, 127], provide background for understanding the work in [83, 60, 19], which performs classification of radio pulsar candidates. The final paper ([86]) uses semi-supervised learning for radio galaxy image classification.

In [37], the authors defined the Hoeffding tree for making VFDTs with streaming data. They prove that, given enough examples, the Hoeffding tree was nearly identical to a batch learner. In a VFDT, the first examples from the stream were used to determine the root 
attribute at each node, with further examples being passed down to the leaves. The Hoeffding bound was used to determine how many examples are necessary to determine a node because it is conservative and independent of the true probability distribution generating the data. The VFDT is versatile, as it can be used with any split evaluation function (InfoGain, Gini coeffeicient, etc.).

The authors in [79] developed puuCVFDT, building on the framework provided by concept-drifting VFDTs (CVFDT) but using only positively labeled and unlabeled samples. CVFDTs for data streams work by recursively replacing all leaves with decision nodes, storing summary statistics in the leaves, and then assigning new data to tree nodes and updating the statistics incrementally. In puuCVFDT, the unlabeled examples are used to estimate negative class conditional probabilities. They showed that puuCVFDT works with uncertain numerical or categorical attributes and performed well in experiments on synthetic and real-life data sets even with 90\% unlabeled data. Results for puuCVFDT varied insignificantly from the fully labeled CVFDT version, but some problems remained. For example, uncertain attribute values are represented by probability distribution functions over ranges, so assigning uncertain samples to tree nodes is problematic. Additionally, it is unrealistic to store all of the samples for multiple scans when building the decision tree, and the Hoeffding bounds cannot be computed in a straightforward way for uncertain data.

In [127], the authors proposed SUN, a semi-supervised classification algorithm with unlabeled data. SUN used the clustering algorithm k-modes to produce concept clusters at leaves in an incremental decision tree (IDT). They showed that it worked well with up to $90 \%$ unlabeled data and could detect concept drift (from noise) by examining deviations between historical and new concepts with a bottom up search. Their experiments used both artificial data and real-world online shopping data from Yahoo. They introduced concept drift rate learning, where the drift rate is the probability that two successive concepts disagree on a random sample. Higher drift rates imply higher error rates, so SUN used information from labeled data to supervise the labeling of relevant unlabeled data based on an IDT, and used clusters generated to track potential concept drifts according to the deviation between new and historical concept clusters. Using error rate and prediction accuracy as performance measures, they compared the performance of SUN to semi-supervised learning decision trees 
CDRDTM, CVFDT, and BagBest. Results showed that SUN had the lowest error rates for classifications and beat out most of the other tested algorithms at detecting concept drifts.

The authors in 83 treated the selection of candidate signals from the SKA pipeline as a binary class stream problem with several challenges: 1) the imbalanced class distribution, 2) RFI changes over time, which they call non-stationarity, and 3) the raw data is unlabeled and cannot reasonably be labeled fast enough. For these reasons, processing needs to be performed in real time. They used the MOA stream mining framework to experiment with Hoeffding trees, which are not intended for unlabeled data. They tried learning only from the stream and pre-training the tree before going online using data from the HTRU survey. They distinguished twenty-two numerical attributes and pre-trained with 1,611 positive and 2,593 negative examples that were randomly sampled for imbalance levels. They compared their classifier to Naïve Bayes, SVM, and C4.5 and tested the four classifiers in static and streaming scenarios. Results showed that recall suffered when facing heavy imbalance, making it unsuitable for solving the candidate selection problem. When pre-training their classifier, their best results by (labeled $\% /$ F-Measure) were: $0 \% / 0.57,50 \% / 0.77,75 \% /$ 0.75 , and $100 \% / 0.75$.

Three main areas concerning big data processing were addressed in [60]: 1) using lowerpower digital processing architectures to make high volume data generation operationally affordable, 2) applying data-adaptive machine learning algorithms for real-time analysis (or "data triage") of large data volumes, and 3) detecting fast radio transient signals in high-rate data streams, including known signals and those that deviate from a standard dispersed-pulse profile. They proposed scalable data archive systems that allow efficient data mining and remote users to run code locally where the data is stored. They introduced the V-FASTR system, which used data from the VLBA, with multiple stations. V-FASTR works by showing separation boundaries for transients, RFI, and noise based on plots of intensity from two widely separated stations, one per axis. They gave a comparison plot of detections vs false positives for semi-supervised, unsupervised, single class SVM, and dedisperion alone. In the plot, the semi-supervised and unsupervised learners have perfect recall with remarkably bad precision, (0.075 and 0.048, respectively). V-FASTR also used a kurtosis filter for RFI filtering before dedispersion, and filters RFI in a parallel pipeline by injecting known pseudo- 
transients and seeing how many telescopes they mask to ensure that the RFI at one station does not dominate the combined sum over all stations [19]. All detections are reviewed by humans through a web portal, and out of the over 150,000 detections generated by the system, less than 10,000 required manual inspection. The system used machine learning with sixteen features, with a multiclass random forest classifier with one class for pulsars and three for types of RFI. They had 7,130 labeled instances, and manually inspect only the top $10 \%$ of pulsar classifications. The classifier is retrained every day on the human-updated set of labeled examples.

Finally, in [86], the authors classified radio galaxy images into three classes by morphology. To accomplish this, they proposed a customized deep convolutional neural network, VGG-X, that performed semisupervised learning in two stages, pre-training and fine-tuning. For pre-training, they applied a self-taught autoencoder to a large unlabeled sample to pretrain the weight and bias parameters of their model. Their intention was to learn the representations of the radio galaxy images and avoid overfitting when learning on a smaller sample. They performed fine-tuning by adding layers to the end of the pre-trained encoder and using the resulting network as a classifier. In experimental comparison to a both a supervised and transfer learning version of VGG-X, the semi-supervised version had the highest F1-scores $(0.89-0.92)$. 


\section{Chapter 4}

\section{Dispersed Pulse Group Identification \& Classification: Methods}

\subsection{Introduction}

This chapter presents a novel, two-stage approach to automatic discovery of transient radio signals within the candidates produced by singlepulse searches 11 These transient signals are in the form of dispersed pulse groups (DPGs), which are collections of pulses appearing as peaks in the signal-to-noise ratio (SNR) vs dispersion measure (DM) subplot of a candidate plot, such as the one shown in Figure 2.3. Note that a DPG is different from a candidate in the traditional sense. A single candidate plot could potentially have many identified DPGs, since a DPG is any local peak in the SNR vs DM subplot. For the first stage, DPG identification, we present a new Recursive Algorithm for Peak IDentification (RAPID) which effectively identified pulsar signals. Individual DPGs, along with their characteristic features, served as instances for machine learning. For the second stage, DPG classification, we created binary and multiclass machine learning models to classify DPGs as originating from pulsar sources or non-pulsar sources. In the following chapter, we describe the application of our new methodology to real-world radio astronomy data.

Our approach consists of two stages: DPG identification using a novel peak identification algorithm and DPG classification using machine learning with imbalance considerations.

\footnotetext{
${ }^{1}$ The work in this chapter was originally published in [34].
} 
Furthermore, each stage requires the completion of several distinct steps. Figure 4.1 provides an illustration of this process. In this section, we first provide a general overview, followed by detailed descriptions of each step in its own subsection, as well as the challenges encountered along the way and how they were overcome.

The first stage of our approach involves collecting and transforming data from the candidates of singlepulse searches into DPGs which effectively characterize actual pulsars. To accomplish this, the data must first be pre-processed, then subsequently scanned for distinct DPGs. The details of our data pre-processing steps are described in Section 5.2. We then identified DPGs using the RAPID algorithm. RAPID scans the data comprising each singlepulse candidate to identify DPGs. This process is detailed in Section 4.2.

For the second stage of our approach, we conducted a series of machine learning trials using supervised learning. Section 4.3 explains how we extracted characteristic features from the DPGs to serve as the input for machine learning algorithms. Section 5.3 describes how we discovered the known pulsars represented in our full set of DPGs and combined them with randomly selected, non-pulsar DPGs to create a benchmark data set. Once we had a benchmark, we used it to evaluate six different machine learning algorithms. Section 4.4 describes our classifiers and how they were used to build intelligent models to classify each DPG. Additionally, due to the extremely small number of pulsars compared to noise and RFI, we addressed the problem of imbalance in the data sets. Imbalance can often bias learners toward the majority class, which is almost always the class of least interest. To examine this problem, we compared results from training models on the unbalanced benchmark to those obtained by using three different imbalance treatments. Section 4.4 .2 presents the challenges of imbalance and the methods we used to overcome them. We evaluated our algorithm according to traditional performance measures described in Section 2.2.4, to select the best performing classifier, then used the models created from the benchmark set to classify each member of the full data set of DPGs identified in the first stage. All DPGs automatically classified as pulsars by machine learning algorithms were then manually inspected to find the number of true positives (discoveries of unknown pulsars and detections of known pulsars) and false positives (non-pulsars incorrectly classified as pulsars). 


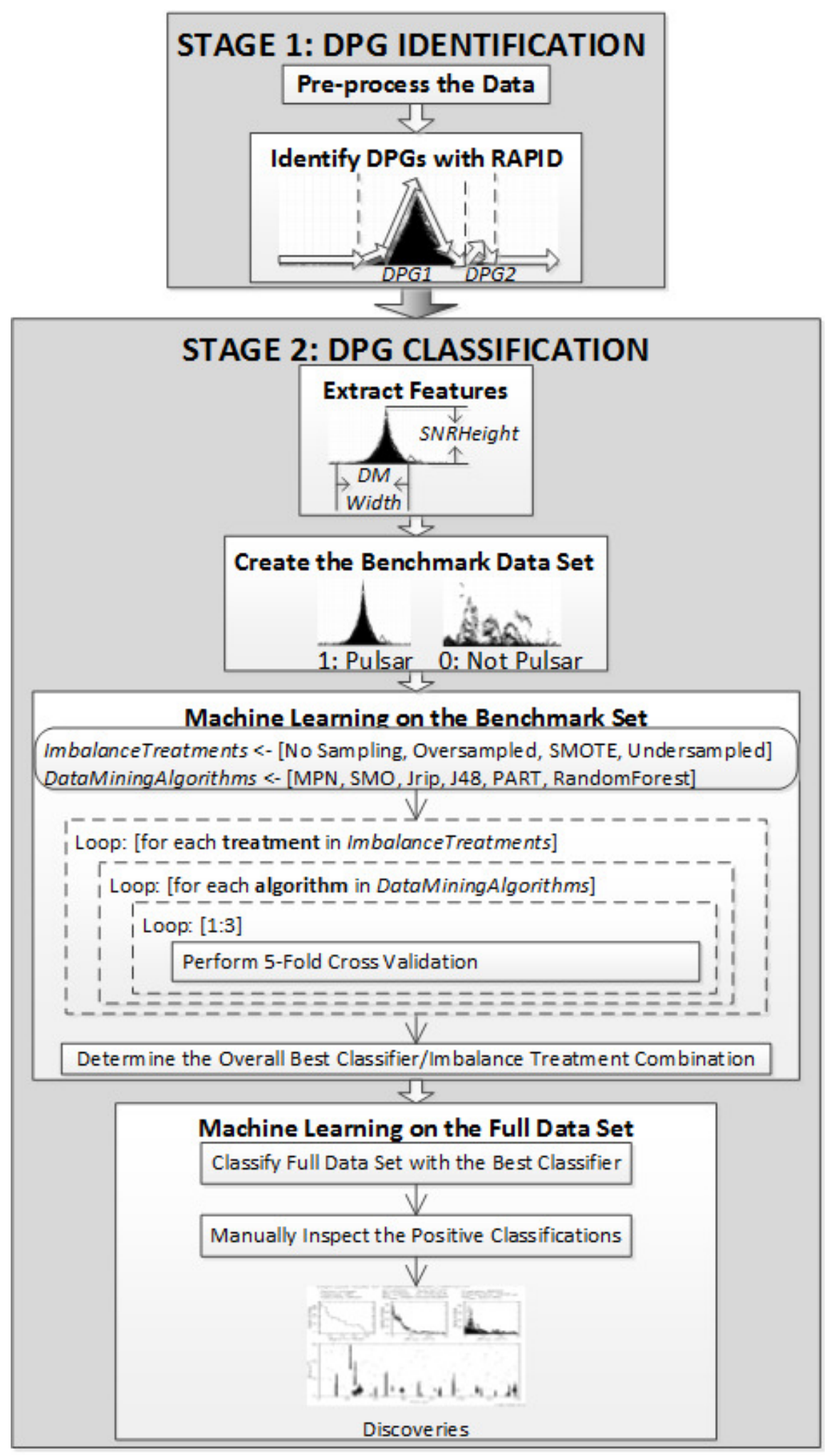

Figure 4.1: Our new machine learning approach to singlepulse detection. 


\subsection{DPG Identification with RAPID}

We propose a simple, recursive peak identification algorithm, RAPID, which divides its input into bins and performs linear regression [1] to fit a straight line to the points within each bin. The slopes of the fit lines for the bins are used to identify the larger slope trends of a DPG. In this context, a DPG is an instance of a peak and its surrounding decreasing values in the data used to create the integrated "SNR vs DM" subplot of a candidate plot, as in Figure 2.3. Note that a single candidate plot can contain many different DPGs, and only one of those DPGs will actually represent a pulsar. At this stage of our work, RAPID looks only at the maximum SNR values for each DM, not at individual pulses.

RAPID can be tuned by adjusting two parameters, the bin size and the slope threshold. The bin size determines how smoothed the detected slopes will be. A smaller bin size allows the identification of narrower DPGs that could be missed by large bin sizes, but at the cost of increasing the size of the output and potentially missing wider DPGs. Using larger bin sizes smooths the data to ignore tiny fluctuations resulting from noise, but may miss smaller DPGs. The slope threshold is a limit placed on the rate of change between the maximum integrated SNR and the DM, and defines the minimum fit-line slope (FLS) required to consider a bin's trend as increasing or decreasing. Higher values will require steeper slopes for DPG recognition, and lower values will allow the detection of more gradual slopes. Strictly speaking, the SNR vs DM curve for a particular pulsar is dependent on the width, observing frequency, and distance from the central DM $(\delta \mathrm{DM})$ of the pulse (as given by Equation 4.1). However, at this point the width of the DPG is not known and we need an initial guess for the slope to begin our search. We set the slope-threshold at 0.5 so as not to exclude any gently sloping pulsars and still be able to identify those with steeper slopes.

For each DPG, RAPID identifies: 1) the start, the starting DM of the first bin to have a positive FLS greater than the slope threshold and immediately following two or more flat bins (bins with FLSs below the slope threshold) or one bin with a negative FLS, 2) the peak, or maximum value between the start and the end, and 3) the end, the starting DM of either the first single bin with a positive FLS or the first of two flat bins seen after the peak. Each bin FLS can take one of three values, depending on the slope threshold: 1 - 
positive and steeper than the slope threshold, 0 - shallower than the slope threshold, or -1 - negative and steeper than the slope threshold. In this way, the algorithm determines if it is climbing or descending a DPG, if it has crossed the peak yet, or if it is on level ground. For example, if the preceding bin had an increasing slope, and the current bin's slope is decreasing, RAPID knows that it has climbed up to a peak and is now descending. If the next two bins were both below the slope threshold, then the algorithm would know that it had reached a termination point and would record the relevant data from the start to the end. By using sloping trends to find the starting and ending points of DPGs, RAPID can identify DPGs of various widths in only one pass through the data.

For each bin, the algorithm passes three values: starting $D M$ - used to determine the next bin, current FLS - for comparison to the next bin's FLS, and status - keeps track of whether the signal has begun ascending and whether it has crossed a peak yet. An algorithm which is designed to reference itself is called recursive. Recursive algorithms divide large problems into many smaller problems and then combine the results. For RAPID, the larger problem is to find a DPG which may span any number of bins. To solve the larger problem, RAPID considers the smaller problem of sloping trends in the $n$th and $n-1$ th bins. With each recursive call, the algorithm passes three values: starting $D M$ - used to determine the next bin, current FLS - for comparison to the next bin's FLS, and status - keeps track of whether the signal has begun ascending and whether it has crossed a peak yet. Recursive code is more efficient in terms of memory and execution time than non-recursive methods for handling certain types of problems. To illustrate this, we developed a non-recursive implementation of RAPID (using 'while' loops) and performed profiling experiments to compare the techniques. The recursive version ran, on average, 4.94 times faster than the non recursive version and used 8.57 times less total memory to identify DPGs in the same 100 pointing data sets.

RAPID is similar to the "momentum" peak identification technique proposed by [54] and described in Section 3.3. However, while their momentum technique relies on the instantaneous rate of change at a point, RAPID uses the slope of regression lines for bins of data points. By breaking the data into bins, we eliminate the need for fitting a more complex equation, and calculations of its derivative, that could be thrown off by noise or RFI. We also ensure that small fluctuations do not affect the overall trends. 
RAPID also differs from other binning techniques for burst detection or peak identification [77, 48, 62] in several key ways. First, all other binning techniques look at only a single value for each bin. If applied to DPG identification, one could use some value, say the mean, to represent the bin. However, this cannot tell us which direction the points inside one bin are trending. Additionally, RAPID only needs to make one pass through the data, while LFA [77] and MEPSA [48] perform an initial pass to identify candidates and then another pass to search for patterns.

Finally, RAPID is designed for a multi-threaded implementation to allow parallel exe-

cution. Since the data for each sky position are independent, RAPID can be instantiated in multiple threads to process the data from multiple positions simultaneously. The output from each scan for DPGs is saved individually and the results are aggregated when all scans are completed.

\subsection{Feature Extraction}

Once RAPID identifies a DPG, our code automatically extracts features to characterize it. The features are extracted from the data used to produce two subplots shown in Figure 2.3 ; the number of pulses (pulse counts) vs DM histogram and the SNR vs DM diagram, and are listed in Table 4.1. The features include measures of width and height, integrations to give an idea of the total "strength" of the DPG, and average values for the DPG.

The last three features in Table 4.1 describe how well a DPG's shape in the SNR vs DM space fits the ideal theoretical shape of a single dispersed pulse [29]. Theoretically, the flux, which is proportional to the SNR, at some offset from the true DM, $\delta \mathrm{DM}$, will follow Equation (4.1). Note that Equation (4.1) describes the shape of a single dispersed pulse, not a DPG. However, typically a group of dispersed pulses will be dominated by its brightest member, making a fit comparison to Equation 4.1 relevant.

$$
\frac{S(\delta \mathrm{DM})}{S}=\frac{\sqrt{\pi}}{2} \zeta^{-1} \operatorname{erf} \zeta
$$

In Equation 4.1, $S(\delta \mathrm{DM}) / S$ is the ratio of the observed flux to the peak flux, erf $\zeta$ is the 
Table 4.1: Features extracted for each DPG and used by machine learning algorithms for classification. Features $5-8$ were taken from data in the Pulse Counts vs DM plot in Figure 2.3, while the rest of the features were taken from the SNR vs DM plot in Figure 2.3.

\begin{tabular}{|c|c|c|}
\hline & Feature & Description \\
\hline 1 & StartDM & The starting DM of the DPG. \\
\hline 2 & StopDM & The ending DM of the DPG. \\
\hline 3 & DMWidth & StopDM - StartDM, or the width in DM of the DPG. \\
\hline 4 & MaxPulseCount & The maximum number of pulses occurring at a DM in the DPG. \\
\hline 5 & IntegratedPulseCount & The total number of pulses counted in the DPG. \\
\hline 6 & AvgPulseCount & $\begin{array}{l}\text { The mean number of pulses detected per DM increment in the } \\
\text { DPG. }\end{array}$ \\
\hline 7 & PulseCountLocalPeak Height & $\begin{array}{l}\text { MaxPulse Count - AvgPulseCount, or the height of the peak above } \\
\text { the local average count of pulses in the DPG. }\end{array}$ \\
\hline 8 & PulseCountPeakDM & The DM corresponding to the maximum pulse count in the DPG. \\
\hline 9 & $\operatorname{Max} S N R$ & The local maximum of the SNR values. \\
\hline 10 & IntegratedSNR & The sum of all SNRs recorded over the DPG. \\
\hline 11 & $A v g S N R$ & The mean SNR value detected per DM increment in the DPG. \\
\hline 12 & SNRLocalPeakHeight & $\begin{array}{l}\text { MaxSNRHeight-AvgSNR, or the height of the SNR peak above } \\
\text { the local SNR average in the DPG. }\end{array}$ \\
\hline 13 & SNRPeakDM & The DM corresponding to the maximum SNR value in the DPG. \\
\hline 14 & FittedMaxSNR & The fitted value for $S$ in Equation 4.1 . \\
\hline 15 & FittedWidth & The fitted value for $w$ in Equation $\overline{4.1}$. \\
\hline 16 & $S N R M a x \chi^{2}$ & $\begin{array}{l}\text { The } \chi^{2} \text { of the maximum SNRs recorded for the DPG against the } \\
\text { ideal distribution, as per Equation } 4.1\end{array}$ \\
\hline
\end{tabular}


error function, and $\zeta$ is the value given by:

$$
\zeta=6.91 \times 10^{-3} \delta \mathrm{DM} \frac{\delta \nu}{w \nu^{3}}
$$

where $\delta \nu$ is the total bandwidth in $\mathrm{MHz}, \nu^{3}$ is the cube of the central frequency in $\mathrm{GHz}$, and $w$ is the full width in ms of the pulse at half of $S$ (FWHM).

We quantified how well each given distribution of points fits the theoretical shape by performing a non-linear least squares regression using Gauss-Newton optimization 2 , and required the difference between the root mean squared error of the current and previous iterations to be less than $10^{-4}$. We used the regression line to estimate $S$ and $w$ for each DPG and then compared the actual fitted curve to the expected theoretical curve by computing the $\chi^{2}$ value. Figure 4.2 provides an example plot of the fit line found for the DPG representing the known pulsar J1645-0317.

The features extracted for all DPGs identified by RAPID were saved in a data set referred to as the full data set throughout this chapter.

\subsection{DPG Classification with Machine Learning}

This section describes how we implemented multiclass classification for DPG classification, how we dealt with the imbalance inherent to the data, and how we evaluated the performance of our classification models.

\subsubsection{Multiclass Classification}

Binary classification occurs when the class variable can assume one of two values, e.g. pulsars and non-pulsars. In multiclass classification, more specialized models can be created by training on multiple classes, each consisting of similar instances. In addition to binary classification models, we also used multiclass versions of the learners presented in Section 2.2.2. To accomplish this, we divided our training examples into four classes based on their appearances: pulsars, very bright pulsars, RRAT-like pulsar or FRB, and non-pulsars. Each DPG

\footnotetext{
${ }^{2}$ We originally used a Levenberg-Marquardt optimizer, but it consistently required thousands of iterations to converge. The Gauss-Newton optimizer converged much more rapidly, drastically reducing the computation time.
} 


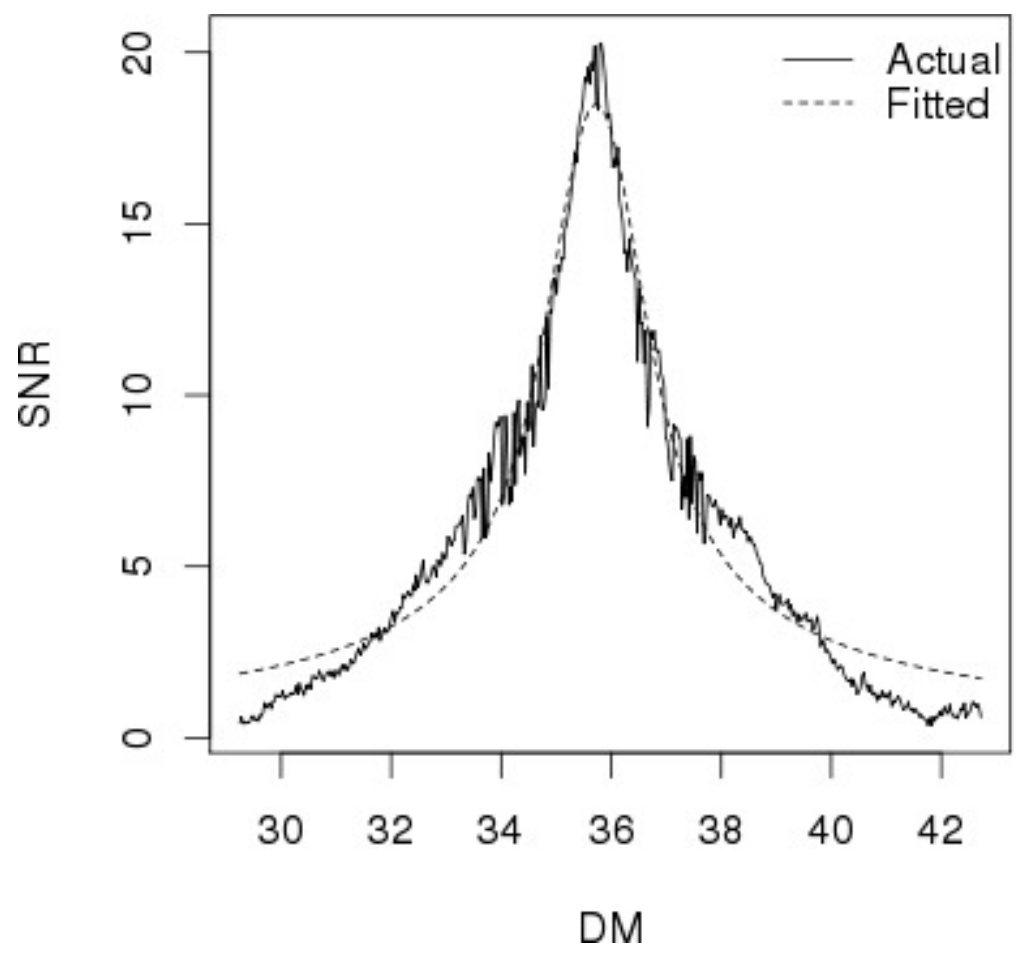

Figure 4.2: The maximum SNR values (solid line) overlaid with the calculated fit (dashed line) according to Equation 4.1 for the DPG representing the known pulsar J1645-0317.

can belong to a candidate plot of one of these four classes. Figure 4.3 provides examples of each class of candidate plot. Compared to pulsars, plots for very bright pulsars are often missing the brightest pulses at the DM of the pulsar, resulting in a flatter distribution at the peak of the SNR vs DM subplot. This is due to PRESTO's single_pulse_search.py clipping the bright pulses. While RRAT-like pulsars have the same shape as pulsars in the SNR vs DM plot, their lack of sustained emission causes them to have lower values for certain metrics, such as IntegratedSNR. FRBs appear similar to RRAT-like pulsars, with only one pulse at high DM.

\subsubsection{Imbalance Considerations}

In data classification, the majority of data gathered is often not very interesting, (e.g. regular usage in network security or financial transactions) or it is mostly interference or noise (e.g. RFI in pulsar searching). When a data set has a very skewed distribution of class variables, it is said to be imbalanced. For our data, out of more than 1.5 million instances 
Pulsar
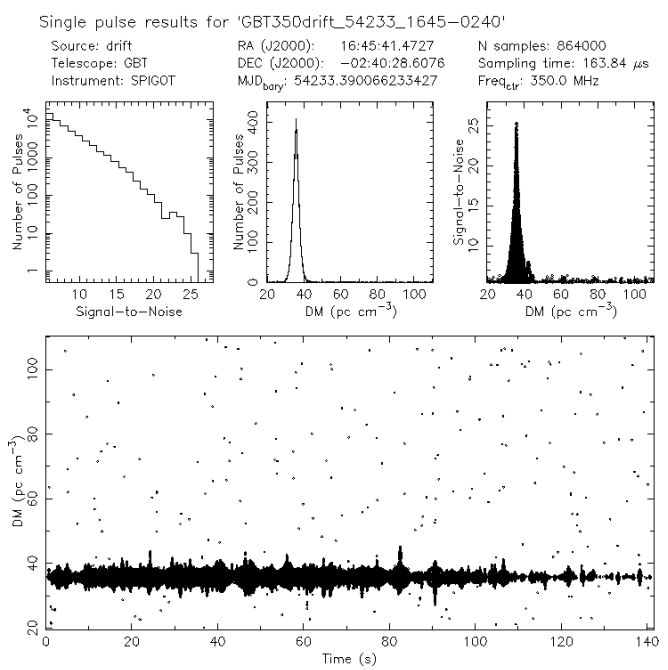

RRAT-like pulsar or FRB
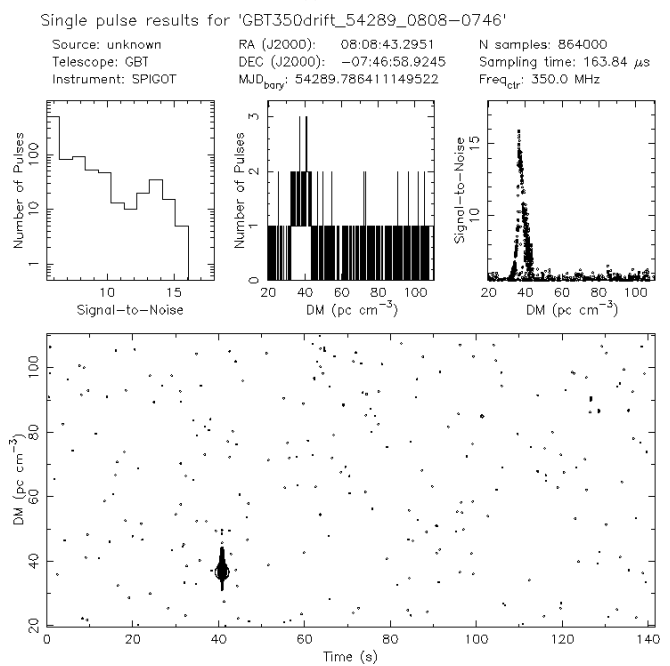

Very Bright pulsar
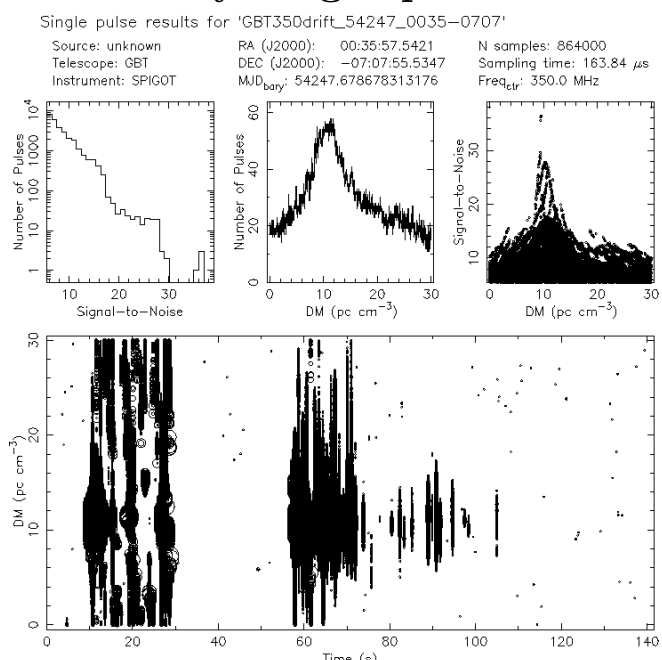

Non-pulsar
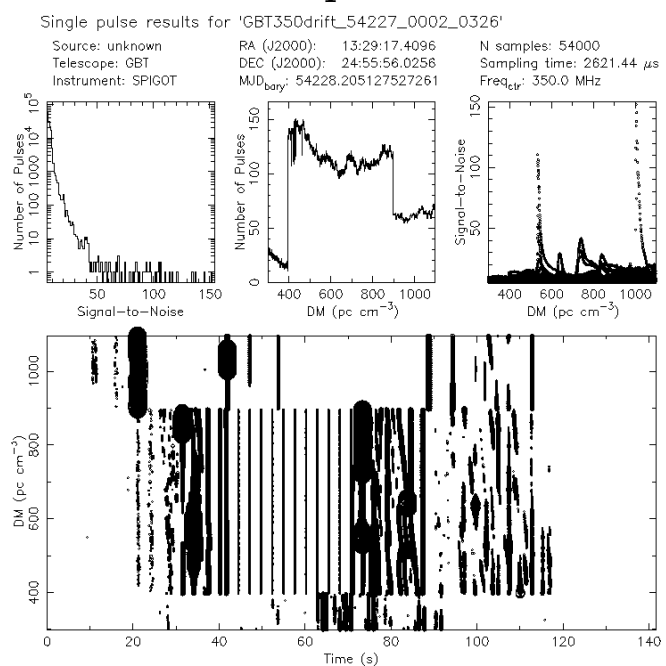

Figure 4.3: Four classes of pulsars based on the appearances of their candidate plots. 
in the full data set, only 327 were positive examples of the target class. The benchmark data set with no imbalance treatment consisted of the 327 positive examples with 9,673 randomly selected and manually validated negative examples. With such a minuscule ratio of minority to majority class members, many learners will "over-train" on the majority class. Therefore, we also considered three versions of the benchmark with three different imbalance treatments:

- Oversampled - Random selections are made from the minority class (with replacement, i.e., the same example may be chosen multiple times) in order to improve the balance between the minority and majority class.

- SMOTE - Synthetic Minority Oversampling TEchnique is similar to oversampling, but each time a random member of the minority class is selected after the first, a synthetic instance is created with small, random perturbations in the values of each of its features. This technique was designed to help eliminate the problem of overfitting a learner to the minority class members that are oversampled [23].

- Undersampled - A traditional treatment to the imbalance problem, where a random sample of the majority class is combined with all instances of the minority class [24].

\subsubsection{Learning Process}

We evaluated the performance of the six learners shown in Table 4.2 on the imbalanced benchmark data set described in Section 5.3 and on three additional benchmark data sets created using the imbalance treatments described in Section 4.4.2. We use the term classifier to refer to the combination of a machine learning algorithm trained on a specific benchmark data set.

For the evaluation, we chose five fold cross-validation, which divides each benchmark version into five folds. The folds contain stratified random samples, i.e., the positive examples are divided equally among them. Four folds were used to train the learner (the "training set") and the fifth was used to test the learner's classifications (the "test set"). Five trials were performed with a different fold serving as the testing set for each trial. 
Table 4.2: The name and type of each machine learning algorithm used for this work.

\begin{tabular}{||c|c|}
\hline \hline Learner & Type \\
\hline MPN & Artificial Neural Network \\
SMO & Support Vector Machine \\
JRip & Rule \\
J48 & Tree \\
PART & Rule + Tree \\
RandomForest & Ensemble Tree \\
\hline \hline
\end{tabular}

When using oversampling imbalance treatments with cross-validation, precautions must be taken to maintain mutual exclusion between the training and testing sets. Otherwise, the same positive examples may exist in both the training and testing sets and the learners may falsely appear to perform very well in the testing environment because they are not being tested on unseen data. We avoided this by first dividing the data into folds and then applying the imbalance treatment only to the training set, and testing the learner on the fifth, unchanged fold which was held out as a testing set. The advantages of performing evaluations in this manner are that all observations are guaranteed to be used for both training and testing, learners are tested on unseen data, and each observation is used for testing exactly once.

\subsection{Conclusions}

In this chapter, we presented the first machine learning approach to pulsar classification in single pulse searches. The approach consists of two main stages: DPG identification and DPG classification. The main contribution of this chapter is the algorithmic development of RAPID, a novel peak detection algorithm for DPG identification.

In Chapter 5, we present the results from an experimental evaluation of our approach using six machine learning algorithms and three imbalance treatments for DPG classification. 


\section{Chapter 5}

\section{Dispersed Pulse Group Identification \& Classification: Evaluation}

\subsection{Introduction}

To empirically evaluate how well our DPG machine learning approach can identify and classify pulsar candidates, we performed a series of experiments using our approach on realworld radio astronomy data. In this chapter, we present the results of these experiments.

\subsection{Data Pre-processing}

The goal of data pre-processing is to reduce the amount of memory required to store the data and increase the efficiency of data access. Our initial data consisted of output from Presto's single_pulse_search.py on data from the GBT drift-scan. The data were composed of individual files for 5,766 DMs (ranging from $0-1,096 \mathrm{pc} \mathrm{cm}^{-3}$ ) for 42,405 separate observation positions. Each file contained data describing the SNR, the downfact (a proxy for pulse width), and the time of each single pulse event for that particular DM. These 244.5 million files encompassed 118 GB of data which required over 922 GB of storage space. Since single pulse detections are often sparse, many of these files contained little or no data. However, each empty or sparse file still required four KB of storage space due to

\footnotetext{
${ }^{1}$ The work in this chapter was originally published in [34].
} 
the minimum allocation size of most hard drives. From the 5,766 files for each observation position, we extracted only the data necessary for our research and created four large files, one for each data structure used by RAPID: number of pulses vs DM, SNR vs DM, maximum SNR vs DM, and DM vs time. This effectively eliminated data bloat by reducing the actual data size by a factor of 3.17 (from 118 GB to $37.2 \mathrm{~GB}$ ), and the storage space required by a factor of 24.6 (from 922 GB to 37.5 GB). Additionally, runtime efficiency was improved by eliminating the need to parse text files and reducing the number of read operations to different locations on the hard drive.

\subsection{Creating the Benchmark Data Set}

For the work described in this chapter, we used a supervised learning approach for classification. This requires training on a fully labeled data set where the class value of every instance is known a priori. For validation, every instance must be manually inspected. The size of our full data set prohibited the labeling and use of all the instances. Instead, we identified as many DPGs representing known pulsars as possible, and combined them with a random sample of manually validated non-pulsar DPGs from the full data set. To select the DPGs from our full data set that corresponded to known pulsars, we compared with the positions and DMs of the 2,234 pulsars listed in the ATNF Pulsar Catalog [87 $]^{2}$ to identify 317 separate observations of 48 distinct pulsars. Using the RRATalog ${ }^{3}$, we were also able to identify ten observations of nine distinct, known RRATs.

We combined these 327 known pulsar DPGs with a random sample of non-pulsar DPGs to create a fully labeled, benchmark data set of 10,000 total instances. We then used the benchmark data set to build and evaluate our machine learning classification models, as described in Section 5.4.1. Finally, we used the classification models with the best performance on the benchmark to classify every instance in the full data set (see Section 5.4.2).

\footnotetext{
${ }^{2}$ http://www.atnf.csiro.au/people/pulsar/psrcat

${ }^{3}$ http://astro.phys.wvu.edu/rratalog
} 


\subsection{Results}

In our experiments, we assigned RAPID a bin size of 25 points and a slope threshold of 0.5. We chose these parameters for our initial study for the following reasons: (a) the bin size was large enough to smooth over noise, yet small enough to detect our DPG examples, (b) the slope threshold was shallow enough to catch the more gradual slopes of some of the wider DPG examples, and (c) our preliminary experimentation with these values identified most known pulsar signals in our data. We ran RAPID with these parameters over the 42,405 observations from the GBT drift-scan survey, which resulted in 1,578,789 DPGs. Since a DPG is any noticeable peak in the DM vs SNR subplot of a candidate plot, and there are many such peaks, there are significantly more DPGs than observations. We intentionally selected a bin size and slope threshold that resulted in a large number of DPGs in order to decrease the probability of missing any pulsars.

This section is divided into three subsections. First, we present the results from training and testing our learners on the four versions of the benchmark data set. Next, we present the results from classifying each DPG in the full data set using our best classifiers. Finally, we compare the results based on one of the best classifiers with results produced by a simple threshold ranking system.

This section uses the following notation to refer to a given classifier:

$$
[\text { learner }]_{[\text {treatment }]}^{[\text {classes }]},
$$

where learner is an abbreviation for the machine learning algorithm, treatment is the imbalance treatment used, and classes is either ' 2 ' for binary classification or ' 4 ' for multiclass classification. For example, the notation $R F_{\text {over }}^{2}$ refers to the classification model created by training a binary class RandomForest machine learning algorithm on the benchmark data set with the oversampled imbalance treatment.

\subsubsection{Benchmark Data Set Results}

We used the six learners shown in Table 4.2 to build classifiers for each of the four versions of the benchmark data set in three repeated trials using five fold cross validation for a total 
Table 5.1: The benchmark results for our classifiers. The center columns report mean values for the performance metrics described in Section 2.2.4. The final two columns report the average time taken to train and test the learners.

\begin{tabular}{||l|r|r|r|r|r|r|r||}
\hline \hline Classifier & Recall & FNR & Precision & FPR & F-M & Train(s) & Test(s) \\
\hline$M P N_{\text {none }}^{2}$ & 0.238 & 0.762 & 0.654 & 0.004 & 0.349 & 16.818 & 0.008 \\
$S M O_{\text {none }}^{2}$ & 0.000 & 1.000 & 0.000 & 0.000 & 0.000 & 3.324 & 0.002 \\
$J R I P_{\text {none }}^{2}$ & 0.571 & 0.429 & 0.680 & 0.009 & 0.620 & 1.269 & 0.005 \\
$P A R T_{\text {none }}^{2}$ & 0.548 & 0.452 & 0.723 & 0.007 & 0.624 & 0.353 & 0.001 \\
$J 48_{\text {none }}^{2}$ & 0.517 & 0.483 & 0.689 & 0.008 & 0.591 & 0.170 & 0.004 \\
$R F_{\text {none }}^{2}$ & 0.459 & 0.541 & 0.918 & 0.001 & 0.612 & 2.901 & 0.053 \\
\hline$M P N_{\text {over }}^{2}$ & 0.867 & 0.133 & 0.199 & 0.135 & 0.324 & 16.301 & 0.017 \\
$S M O_{\text {over }}^{2}$ & 0.602 & 0.398 & 0.120 & 0.172 & 0.200 & 0.545 & 0.016 \\
$J R I P_{\text {over }}^{2}$ & 0.739 & 0.261 & 0.466 & 0.033 & 0.572 & 3.325 & 0.015 \\
$P A R T_{\text {over }}^{2}$ & 0.706 & 0.294 & 0.462 & 0.032 & 0.558 & 0.814 & 0.015 \\
$J 48_{\text {over }}^{2}$ & 0.689 & 0.311 & 0.430 & 0.035 & 0.529 & 0.254 & 0.013 \\
$R F_{\text {over }}^{2}$ & 0.718 & 0.282 & 0.714 & 0.011 & 0.716 & 2.931 & 0.091 \\
\hline$M P N_{\text {smote }}^{2}$ & 0.878 & 0.122 & 0.222 & 0.120 & 0.354 & 16.420 & 0.013 \\
$S M O_{\text {smote }}^{2}$ & 0.749 & 0.251 & 0.104 & 0.251 & 0.182 & 0.199 & 0.012 \\
$J R I P_{\text {smote }}^{2}$ & 0.852 & 0.148 & 0.362 & 0.058 & 0.509 & 3.075 & 0.009 \\
$P A R T_{\text {smote }}^{2}$ & 0.842 & 0.158 & 0.344 & 0.062 & 0.488 & 1.147 & 0.017 \\
$J 48_{\text {smote }}^{2}$ & 0.823 & 0.177 & 0.351 & 0.059 & 0.492 & 0.428 & 0.011 \\
$R F_{\text {smote }}^{2}$ & 0.834 & 0.166 & 0.538 & 0.028 & 0.654 & 5.503 & 0.114 \\
\hline$M P N_{\text {under }}^{2}$ & 0.884 & 0.116 & 0.162 & 0.173 & 0.274 & 1.529 & 0.024 \\
$S M O_{\text {under }}^{2}$ & 0.786 & 0.214 & 0.087 & 0.319 & 0.157 & 0.019 & 0.017 \\
$J R I P_{\text {under }}^{2}$ & 0.896 & 0.104 & 0.205 & 0.135 & 0.334 & 0.073 & 0.014 \\
$P A R T_{\text {under }}^{2}$ & 0.895 & 0.105 & 0.171 & 0.168 & 0.288 & 0.027 & 0.011 \\
$J 48_{\text {under }}^{2}$ & 0.891 & 0.109 & 0.198 & 0.140 & 0.324 & 0.241 & 0.079 \\
$R F_{\text {under }}^{2}$ & 0.927 & 0.073 & 0.287 & 0.090 & 0.438 & 0.241 & 0.079 \\
\hline \hline
\end{tabular}




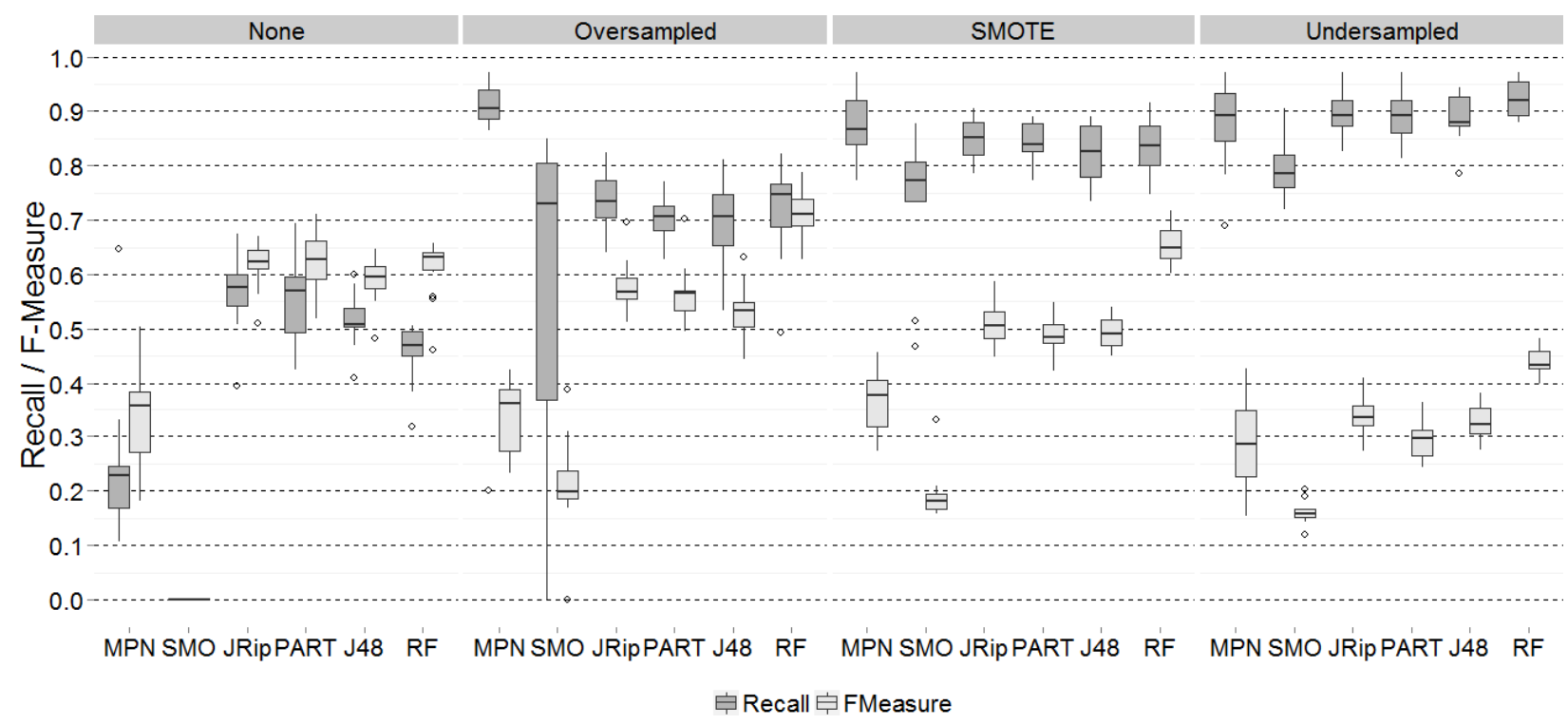

Figure 5.1: The results of binary machine learning trials on the four versions of the benchmark data set. The median of each distribution is the central horizontal line, the shaded areas to either side illustrate the semi-inter-quartile ranges, the lines or "whiskers" extending from each end give the first and fourth quartiles, and outliers are represented by open circles. The distributions of performance metrics (shown on the y axis) for each learner (annotated at the bottom) are organized into four groups, one for each version of the benchmark data set (annotated at the top).

of 360 trials. The trials were conducted using Weka's experimenter on an Alienware M14xR2 with an Intel Core i7 CPU, 16 GB RAM, and a 512 GB solid state drive. The results of the binary classification are displayed in Table 5.1, which includes training and testing times. Figure 5.1 shows boxplots of the distributions of key performance metrics, grouped by the benchmark version.

Based on Table 5.1 and Figure 5.1, we make the following observations:

- All classifiers with imbalance treatments had higher recall values than those with unbalanced data.

- RandomForest provided the highest average F-Measure and good recall values on all data sets with imbalance treatments.

- MPN had the best recall values for the oversampled and SMOTE imbalance treatments, but the second worst F-Measure for all imbalance treatments. 
- SMO had the worst performance for the four benchmark data sets.

The choice of a best classifier from the benchmark trials depends on the most desirable performance measure. For automating pulsar classification, the F-Measure may be considered the most important performance measure because a classifier with a high F-Measure must have good scores for both recall and precision. (As described in Section 2.2.4, a high recall indicates the classifier will correctly classify most positive instances and a high precision indicates the classifier will not result in many false positives.) While a high recall is important, the point of automation is to minimize human involvement. A low precision means that only a small fraction of positive classifications is relevant, that is, there are many false positives which would require manual inspection and therefore is undesirable for this work. For example, although the $M P N_{\text {over }}^{2}$ classifier had the best recall among learners on the oversampled benchmark, it has a very low F-Measure because it produced many false positives. Therefore, with respect to the F-Measure the best classifiers are $R F_{\text {smote }}^{2}$ and $R F_{\text {over }}^{2}$.

Our result indicating that oversampled and SMOTE imbalance treatments provide higher recall values is consistent with the findings of [95], which showed that resampling techniques generally improved results for all learners, and specifically that using oversampling and SMOTE with C4.5 trees provided better classifiers than other techniques. (As mentioned in Section 2.2.2, J48 trees are Java implementations of C4.5 trees.) A RandomForest learner was not used by [95]. C4.5 was also shown to benefit from resampling techniques by [26].

It should be noted that the training and testing times show that MPNs are by far the slowest of the six learners tested. MPNs, as most ANNs, use a gradient descent optimization routine to determine the weighted values between network nodes during back propagation. Gradient descent calculations are computationally expensive, and are often the cause of increased training times. Furthermore, while recall values were very high, the F-Measures obtained for MPNs were consistently lower than all learners except SMO, with a large variance. Note that ANNs are one of the most common machine learning techniques applied to the problem of radio pulsar detection in periodicity searches, and were used in each paper discussed in Section 3.1 that performed machine learning [41, 7, 137, 90]. 
Table 5.2: A comparison of the performance of classifiers using binary RandomForest (RF) and Multilayer Perceptron Network (MPN) learners on the full data set.

\begin{tabular}{||l|r|r|r|r||}
\hline \hline Classifier & PDs & $\mathbf{C K s}$ & $\mathbf{A K s}$ & $\mathbf{F P s}$ \\
\hline$R F_{\text {none }}^{2}$ & 0 & 304 & 5 & 32 \\
$R F_{\text {over }}^{2}$ & 2 & 327 & 15 & 451 \\
$R F_{\text {smote }}^{2}$ & 3 & 326 & 46 & 1,940 \\
$R F_{\text {under }}^{2}$ & 6 & 326 & 33 & 9,750 \\
\hline$M P N_{\text {none }}^{2}$ & 0 & 79 & 1 & 696 \\
$M P N_{\text {over }}^{2}$ & 6 & 309 & 23 & 43,943 \\
$M P N_{\text {smote }}^{2}$ & 3 & 257 & 23 & 14,066 \\
$M P N_{\text {under }}^{2}$ & 6 & 298 & 29 & 110,629 \\
\hline \hline
\end{tabular}

Results from building and testing multiclass learners on the four versions of the benchmark data set were similar to the binary classification results, with the $R F_{\text {smote }}^{4}$ and $R F_{\text {over }}^{4}$ classifiers performing the best with respect to F-Measure.

\subsubsection{Full Data Set Results}

Based on the results reported in Section 5.4.1 we selected the models produced by two learners - RF (best F-Measures) and MPN (best recalls) - in combination with all imbalance treatments to classify every instance in the full data set. Since most of the DPGs in the full data set were not labeled, it was not possible to calculate the same performance metrics as for the benchmark data sets. Instead, we evaluated the performance of the models by the following criteria: how many potential discoveries (PDs) were found, how many known pulsars were classified correctly (CKs), how many additional known pulsars (AKs) were found beyond those included in the benchmark, and how many DPGs classified as pulsars were false positives (FPs), i.e., non-pulsars incorrectly classified as pulsars 4 . Table 5.2 provides the results for all benchmark versions of the binary RF and MPN learners.

Three important results stand out from Table 5.2. (1) RF models had almost perfect CK and 1-2 orders of magnitude fewer FPs than their MPN counterparts. This finding was expected based on the low F-Measures of the MPN learner on the benchmark data sets.

\footnotetext{
${ }^{4}$ Note that not all instances in the FPs column were examined for classifiers with more than 9,000 FPs. Such classifiers were able to achieve high PDs, CKs, and AKs by simply classifying almost everything as a pulsar, which defeats the purpose of automation.
} 
Table 5.3: A comparison of the performance of oversampled and SMOTE multiclass RandomForest (RF) classifiers on the full data set.

\begin{tabular}{||l|r|r|r|r||}
\hline \hline Classifier & PDs & CKs & AKs & FPs \\
\hline$R F_{\text {over }}^{4}$ & 5 & 327 & 32 & 330 \\
$R F_{\text {smote }}^{4}$ & 6 & 316 & 35 & 1,718 \\
\hline \hline
\end{tabular}

(2) Classifiers using MPN learners had lower CK, i.e., they failed to correctly classify from $5-75 \%$ of the known pulsar examples. This result was unexpected, as the classifiers with MPN learners had the highest recall values in the benchmark experiments. (3) The most FPs, for both RF and MPN, were produced in combination with the undersampled imbalance treatment, which is consistent with the lowest precision and F-Measure obtained on the benchmark data set. Upon further inspection, we found that the undersampled classifiers were oversimplified, making them incapable of distinguishing between pulsar examples and non-pulsar examples resulting from a variety of noise and RFI. We attribute this oversimplification to the subsample of negative training examples not being representative of the full distribution, which is an expected outcome when undersampling a severely imbalanced data set.

Based on the results presented in Table 5.2 , we decided to use the $R F_{\text {over }}^{4}$ and $R F_{\text {smote }}^{4}$ multiclass classifiers on the full data set, due to their nearly perfect $\mathrm{CK}$, high $\mathrm{AK}$, and low FP values. $\left(R F_{\text {under }}^{4}\right.$ and $M P N$ classifiers with all imbalance treatments were not used due to the high number of FPs.) Table 5.3 reports the results, from which we make the following observations: (1) The classifiers with multiclass RF learners were superior to their binary counterparts, for both imbalance treatments, because they were trained on three pulsar classes whose appearance and feature values are quite different. Specifically, classifiers using multiclass RF learners were able to detect potential RRATs, like the one shown in Figure 5.2 , which were missed by the binary classifiers. (2) $R F_{\text {smote }}^{4}$ found six PDs, which contained all of the PDs from the other classifiers, with a much smaller number of FPs than the binary classifiers shown in Table 5.2. (3) With respect to imbalance treatments, there is a trade-off to be made. Compared to the oversampled treatment, SMOTE resulted in more detections, both PDs and AKs, but with the added cost of over five times more FPs requiring manual inspection. 
Single pulse results for './GBT350drift_54236_1748+2241_singlepulse/GBT:

Source: unknown

Telescope: GBT

Instrument: SPIGOT

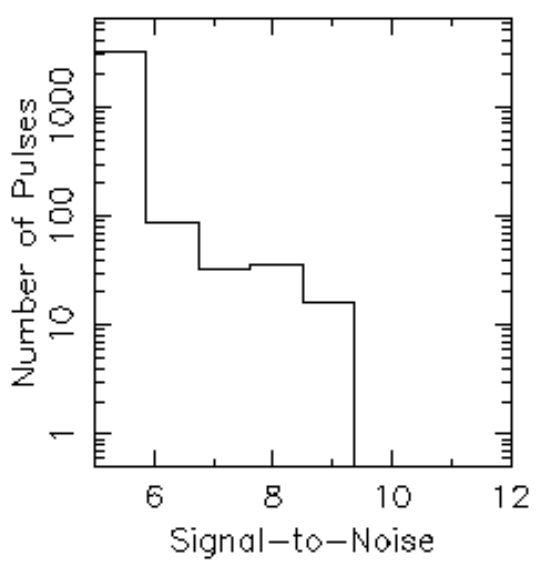

N samples: 864000

Sompling time: $163.84 \mu s$

Freq $_{\mathrm{ctr}}: 350.0 \mathrm{MHz}$

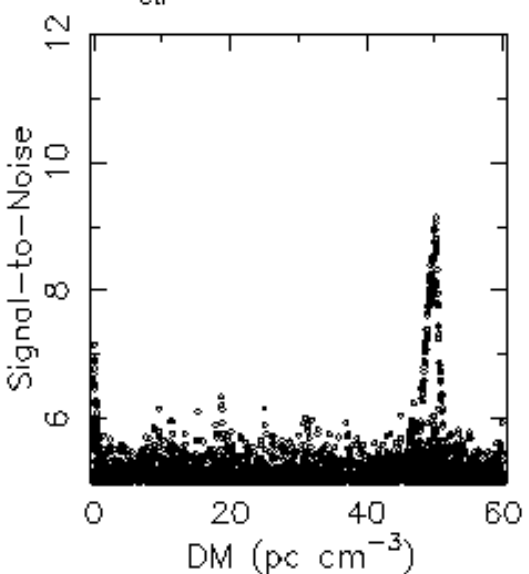

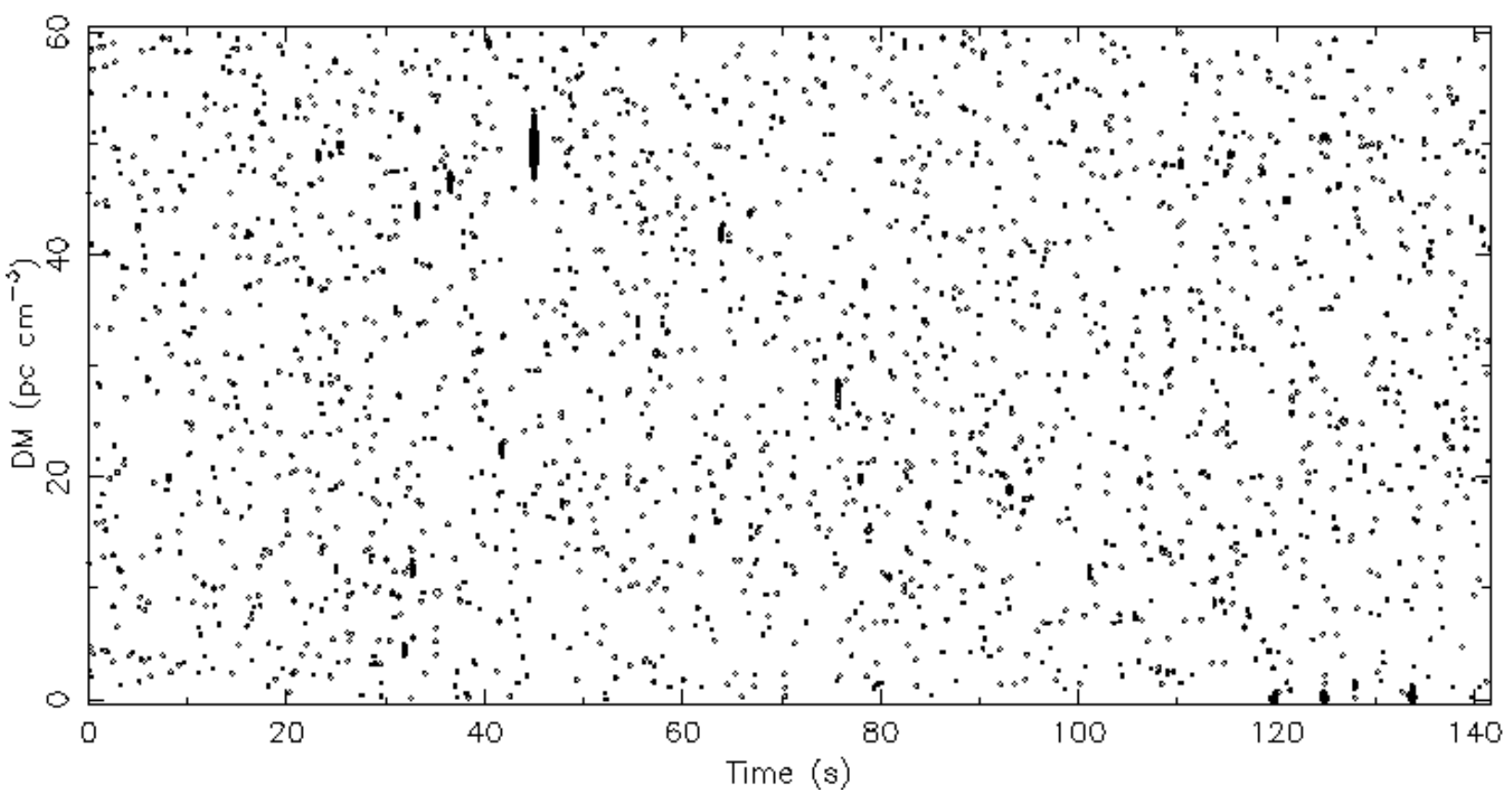

Figure 5.2: One potential RRAT discovery found by our classifiers. 
Table 5.4: Rankings based on a simple threshold for the feature $S N R M a x \chi^{2}$. The first column gives the statistic used as the threshold value, the second column shows the value of $S N R M a x \chi^{2}$ which will be used as the threshold, the third column displays the number of DPGs in the full data set that have a value below the threshold, the fourth column shows how many of the DPGs in the third column are known pulsars, the fifth column gives the percent of known pulsars detected below the given threshold, and the sixth column shows what percentage of the top ranked DPGs are pulsars (PER). For many DPGs, the fitting routine could not reach convergence.

\begin{tabular}{||l|r|r|r|r|r||}
\hline \hline Statistic & Value & \#iValue & \#DPGs & KDR & PER \\
\hline 1st Quartile & 47 & 80,512 & 136 & $42 \%$ & $0.17 \%$ \\
Median & 189 & 161,854 & 266 & $81 \%$ & $0.16 \%$ \\
3rd Quartile & 571 & 242,534 & 312 & $95 \%$ & $0.13 \%$ \\
Mean & 816 & 265,738 & 316 & $97 \%$ & $0.12 \%$ \\
Maximum & $9 \times 10^{6}$ & 323,447 & 323 & $99 \%$ & $0.10 \%$ \\
\hline \hline
\end{tabular}

\subsubsection{Comparison of Our Results to Simple Ranking}

To further evaluate the performance of one of our classifiers, $R F_{o v e r}^{2}$, we compared our results to a simple ranking approach based on the feature $S N R M a x \chi^{2}$, a measure of how well the shape of the DPG in the SNR vs DM plot fits the ideal, theoretical shape of a dispersed pulse given by Equation 4.1] [29].

We sorted all DPGs in the full data set by their $S N R M a x \chi^{2}$ values and calculated summary statistics (1 $1^{\text {st }}$ quartile, median (i.e., the $2^{\text {nd }}$ quartile), $3^{\text {rd }}$ quartile, mean, and maximum) to use as thresholds. We then examined how many DPGs in the full data set had $S N R M a x \chi^{2}$ values less than each statistic, and how many of those DPGs were known pulsars. Note that no ranking system based on $S N R M a x \chi^{2}$ can detect $100 \%$ of the known pulsars in the data set, since the fitting routines for several known pulsars failed to converge due to noise spikes which offset their central peaks.

We report the percentage of known pulsars detected with values below a given threshold, which we call the Known Detection Rate (KDR). To quantify how much effort would produce results if one performed manual inspection of all top ranked DPGs, we also computed what percent of the top ranking DPGs are known pulsars. We call this the Positive Effort Rate (PER). The results are presented in Table 5.4.

As Table 5.4 shows, if the median value of $S N R M a x \chi^{2}$ was used as a threshold, we 
would have to manually inspect over 160,000 DPGs. Only $0.16 \%$ of those 160,000 DPGs would be known pulsars and we would only be able to detect $81 \%$ of the total known pulsars in the data set. In comparison, if we used our binary oversampled RandomForest model, $100 \%$ of the known pulsars will be correctly classified and less than 470 DPGs would require manual inspection. The final column in Table 5.4 shows that with any threshold value, at best, less than $0.2 \%$ of the top ranked DPGs will be known pulsars. Alternatively, our binary oversampled RandomForest model resulted in a PER of $41 \%$. We believe that our machine learning approach outperforms the ranking because the classification models are multivariate, i.e., they take many different features of the DPGs into consideration.

\subsection{Conclusions}

In this chapter, we presented an experimental evaluation of the DPG machine learning approach presented in Chapter 4. Our novel peak identification algorithm, RAPID, successfully identified DPGs, which are local peaks in the output from single pulse searches, and extracted meaningful features to describe them. Then, we used machine learning algorithms with imbalance consideration to classify the identified DPGs, first on a benchmark data set and then on the full, unlabeled data set created based on observations made by the Green Bank Telescope. The benchmark data set was created with over three hundred known pulsar signals and over 9,600 manually validated negative examples. To examine the problem of imbalance, we applied three different imbalance treatments to the original unbalanced benchmark data set. We used these four versions of the benchmark (the original unbalanced version and the three balanced versions) to train and test binary and multiclass versions of six different machine learning algorithms, resulting in 48 classifiers. We found that every classifier using an imbalance treatment provided higher recall values than the classifiers using unbalanced data. The classifiers using the RF ensemble tree learner provided the best overall balance between recall and precision (i.e., the highest F-Measure values). On the other hand, the classifiers we tested using MPNs resulted in the highest recalls, but second worse F-Measures and the longest training and testing times.

Based on these results we selected a subset of classifiers to search for potential pulsar 
discoveries in the full, unlabeled data set. First, we used the set of classifiers using binary RF and MPN learners to classify the instances in the full, unlabeled data set. Every classifier with a RF learner outperformed the best classifier with a MPN learner, resulting in more correctly identified known pulsars and fewer false positives. Additionally, the undersampling imbalance treatment restricted the number of negative training examples, resulting in oversimplified classifiers that had trouble distinguishing between pulsars and noise/RFI in the full data set. Next, we tested multiclass RF learners using oversampling and SMOTE on the full data set. In general, classifiers with multiclass RF learners significantly outperformed binary classifiers. Specifically, they reported as many potential discoveries, were better in detecting potential RRAT discoveries, and produced less false positives than the binary classifiers. The oversampled and SMOTE imbalance treatments each had advantages and disadvantages. While the classifiers with oversampled imbalance treatments perfectly classified all known pulsar examples with very few false positives, they missed potential discoveries that were found by classifiers with SMOTE imbalance treatments. The SMOTE classifiers, however, mis-classified several known pulsar examples and produced four to five times more false positives.

Overall, the combination of the multiclass RF learner with the SMOTE imbalance treatment was the most efficient - it detected six potential pulsar discoveries with less false positives than any other classifier which also detected all six potential discoveries. The potential discoveries are currently under further review. Confirming them will require making frequency-time plots of the raw search data to confirm the broadband nature of any pulses and the expected $\nu^{-2}$ dependence of the dispersive delay, and then performing re-observations of these sky positions.

We compared the oversampled binary RF classifier to a simple ranking method based on a threshold representing how well the actual data fit a theoretical curve. The machine learning approach significantly outperformed this ranking by correctly identifying all known examples and reducing the number of DPGs requiring manual inspection by several orders of magnitude. The machine learning approach and its evaluation presented in Chapters 4 and 5 were published in a prominent journal for astronomy and astrophysics [34]. 


\section{Chapter 6}

\section{Single Pulse Event Identification \& Classification: Methods}

\subsection{Introduction}

This chapter presents an extension of the approach to automatic discovery of transient radio signals presented in Chapter 4 However, instead of targeting DPGs, the primary focus of the work in this chapter is to classify single pulses, which are collections of single pulse events (SPEs) clustered together in the DM vs time space that may represent pulsar emissions. Figure 6.1 shows a customized single pulse search candidate we generated for the known pulsar, B1853+01. In the SNR vs DM (top) and DM vs time subplots (bottom) of Figure 6.1, each individual data point represents a SPE. Single pulses are collections of SPEs appearing as clusters of points in the DM vs time space that have distinct peaks when viewed in the SNR vs DM space. Narrowing our focus from DPGs to individual pulses allows for the identification and classification of signals that are much fainter, or that might be obscured by RFI, when viewed on a larger scale.

In Figure 6.1, we emphasize two individual single pulses, shown in black. The upper left subplot is an SNR vs DM scatterplot scaled to the domain and range of the emphasized single pulses. The upper right and bottom subplots are constructed in the same manner as their corresponding subplots in a standard candidate plot (as in Figure 2.3) with one

\footnotetext{
${ }^{1}$ The work in this chapter was originally published in [35].
} 


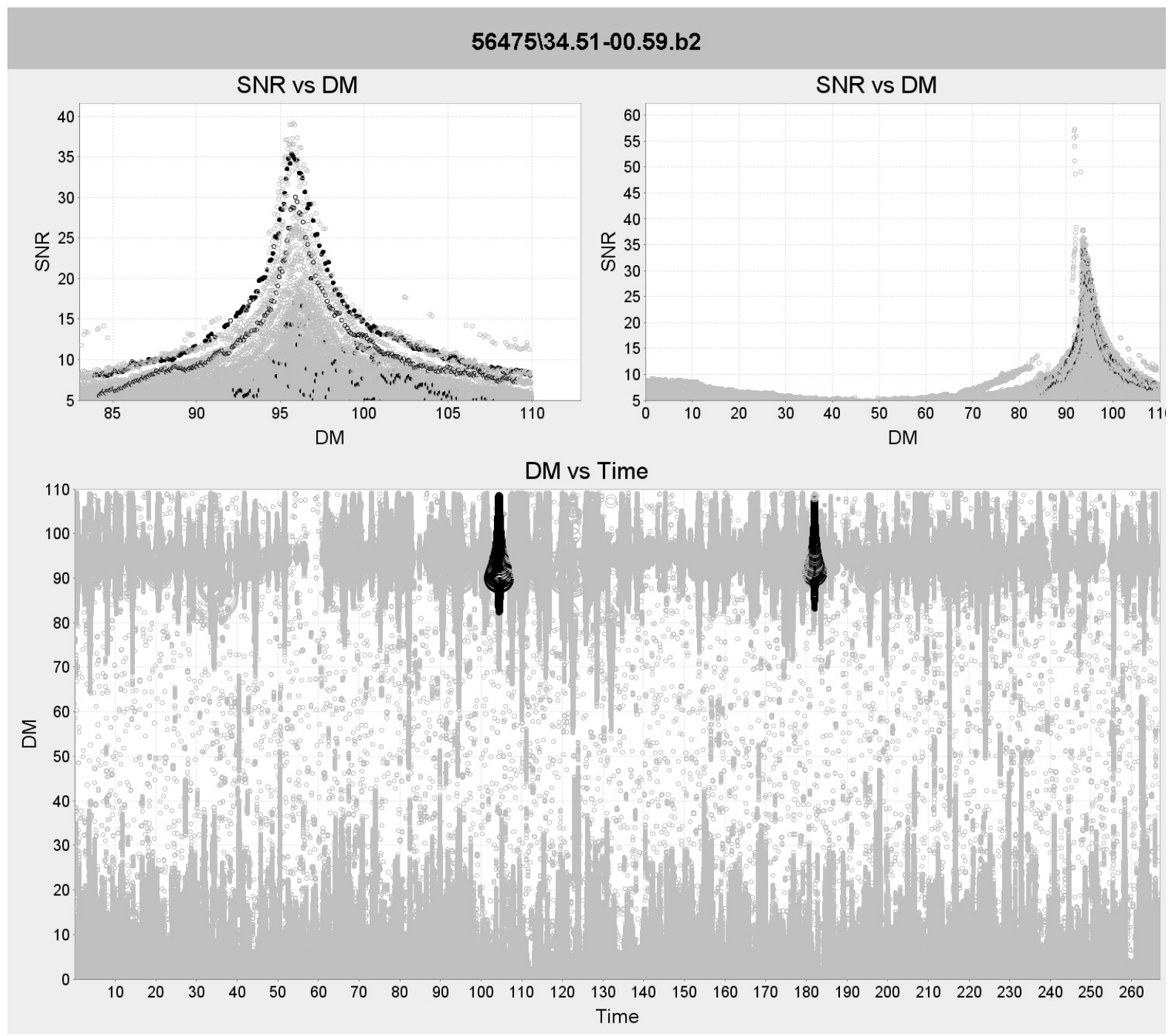

Figure 6.1: The known pulsar B1853+01 identified by a single pulse search. The SPEs emphasized in black belong to two distinct pulses. The subplots, clockwise from the top left, show a zoomed in scatter diagram of the SNRs for each SPE in the of DM range of the emphasized single pulse (in pc $\mathrm{cm}^{-3}$ ), a scatter diagram showing the SNRs and DMs of all SPEs in a standardized DM range, and a scatter diagram showing the DMs and times (in seconds) of each SPE, with the markers for individual SPEs scaled in size by their SNR. 
Table 6.1: Summary information about the SPEs contained by the pulses emphasized in Figure 6.1.

\begin{tabular}{||c|c|c|c|c|c||}
\hline \hline Name & \# SPEs & $\begin{array}{c}\text { DM Range } \\
\left(\mathrm{pc} \mathrm{cm}^{-3}\right)\end{array}$ & $\begin{array}{c}\text { Peak DM } \\
\left(\mathrm{pc} \mathrm{cm}^{-3}\right)\end{array}$ & Peak SNR & $\begin{array}{c}\text { Time Range } \\
(\mathrm{s})\end{array}$ \\
\hline Single pulse\#1 & 352 & $84.0-111.9$ & 96.4 & 37.39 & $104.404470-104.41475$ \\
Single pulse\#2 & 349 & $84.1-109.2$ & 96.5 & 31.78 & $181.996637-182.007571$ \\
\hline \hline
\end{tabular}

difference: only the SPEs contained by the emphasized single pulses are colored black, all other SPEs are grayed out. Table 6.1 provides summary information for the two emphasized single pulses. Single pulse\#1 is brighter than and temporally precedes Single pulse\#2, as shown by their Peak SNRs and Time Ranges in Table 6.1. Single pulse\#1 is the top line of SPEs in the upper left subplot of Figure 6.1 and is the leftmost cluster of SPEs in the DM vs Time subplot.

Our machine learning approach for single pulse identification and classification consists of four stages, as depicted by the scientific workflow in Figure 6.2 [82]. (Note that by "raw data", we refer to data that has already been processed through the first three phases of a single pulse search described in Section 2.1.1.) In stage one, the raw data are pre-processed into a series of SPE files. In stage two, the SPE files are fed into a customized DBSCAN clustering algorithm $2^{2}$ which solves problems specific to radio astronomy clustering, such as the merging of clusters from one single pulse that appear disparate due to artifacts of data processing. After identifying clusters of associated SPEs in the DM vs Time space, we extract characteristic features from them to create a series of files describing the clusters found. The SPE and cluster files are then uploaded to the Hadoop Distributed File System (HDFS). In stage three, which is described in Chapter 8, we use distributed computing to scale up the process of peak identification. Finally, classification is performed in stage four, which consists of two steps. First, we manually inspect single pulses from data containing known pulsars to create two multiclass benchmark data sets (each from a different, real-world data set) to use for supervised machine learning. Then, we use the benchmark data sets to experimentally evaluate 360 different classifiers, each of which is a combination of a machine learning algorithm, a multiclass definition scheme, an imbalance treatment, and a feature

\footnotetext{
${ }^{2}$ The algorithm was developed by fellow team members [97] as part of a larger research effort funded by NSF Award \#1458952. For a brief description of DBSCAN, see Section 2.2.1
} 


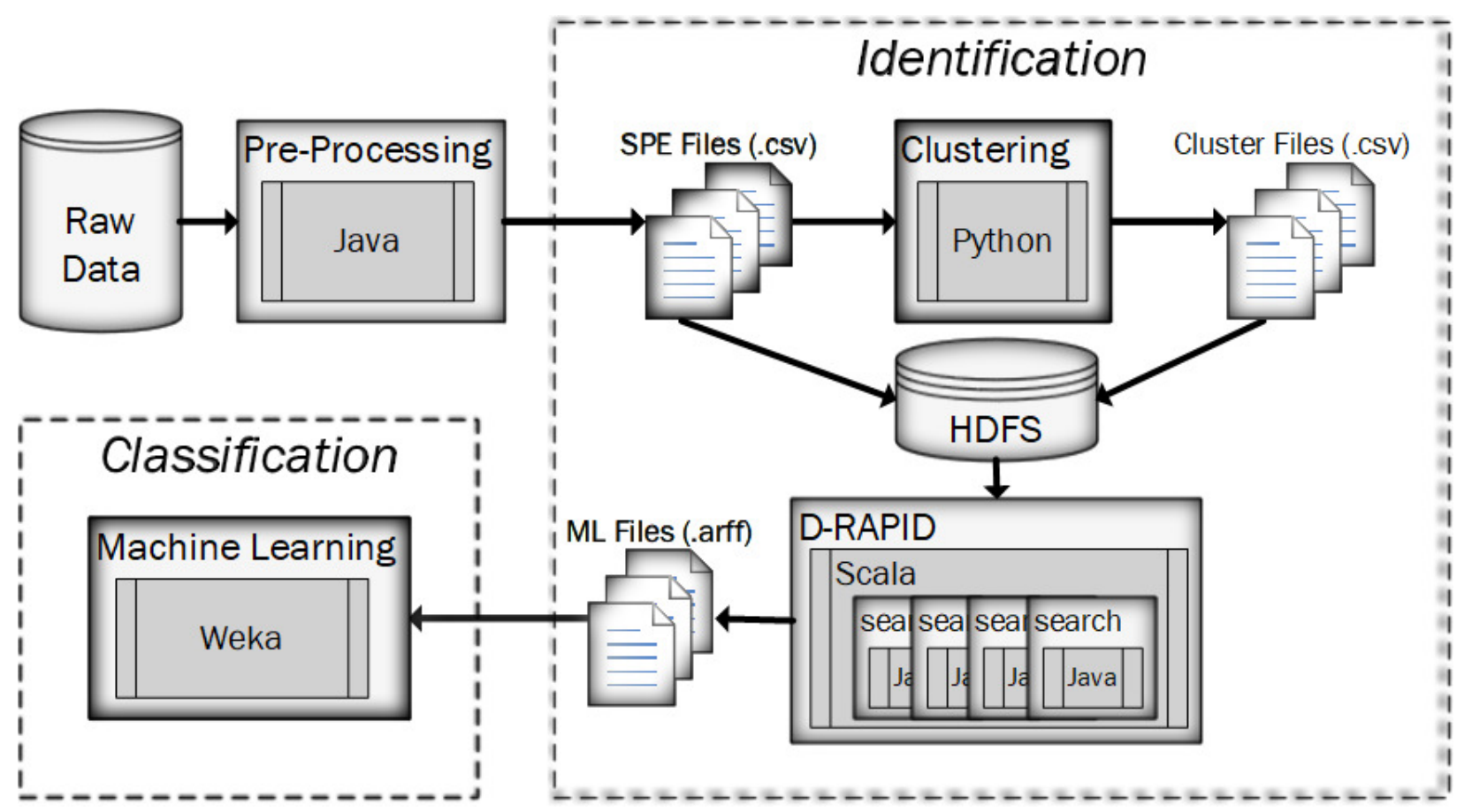

Figure 6.2: The scientific workflow of our scalable approach to single pulse identification and classification.

selection method. Chapters 6, 7, and 8 are focused on stage four with the goal of improving the execution performance of single pulse classification (8.2).

The machine learning approach presented in this chapter focuses on the classification of single pulses in clusters of SPEs, rather than DPGs in aggregated data, as in Chapter 4 . This required the use of a clustering method and extensive alteration to our peak detection algorithm. Additionally, we approached multiclass classification in a new way. Instead of grouping single pulses into four classes based on how their candidate plots look to the eye, as in Section 4.4.1, we based our classes on two physical properties of the signals: average brightness and distance from Earth. We also performed extensive experimentation with different feature selection methods designed to eliminate extraneous or irrelevant features before training classifiers. 


\subsection{Feature Extraction}

In Chapter 4, we described the extraction of a set of 16 features to be used by classifiers to distinguish pulsar single pulses from non-pulsar single pulses. Focusing on individual clusters of SPEs allowed the inclusion of several additional features, which are given in Table 6.2 . The first four features are specifically defined for single pulses and are calculated from the data used to create DM vs. Time subplots, like the one in Figure 6.1. The last two features could be extracted for either DPGs or single pulses.

Table 6.2: Additional features extracted for each cluster and used by machine learning algorithms for classification.

\begin{tabular}{|c|c|}
\hline Feature & Description \\
\hline StartTime & The arrival time of the first SPE in the cluster. \\
\hline Stop Time & The arrival time of the last SPE in the cluster. \\
\hline ClusterRank & $\begin{array}{l}\text { An SNR based ranking of the cluster compared to others } \\
\text { in the same observation }([97]) \text {. }\end{array}$ \\
\hline PulseRank & $\begin{array}{l}\text { The rank of a particular peak when compared to other } \\
\text { peaks found in a cluster and ordered by SNRMax. }\end{array}$ \\
\hline DMSpacing & The interval between two consecutive DM values. \\
\hline SNRRatio & $\begin{array}{l}\text { The ratio of the SNR of the first point in the peak to the } \\
\text { maximum SNR. }\end{array}$ \\
\hline
\end{tabular}

We included the DMSpacing because it affects how single pulses or DPGs appear in different DM ranges, as noted by [97]. The DMSpacing is the minimum difference between two consecutive DM values, and increases from 0.01 for low DM values to 2.00 for very high DM values in the DM vs. time space. Since D-RAPID calculates the slope of a linear regression through the points of a bin, differences in scaling on the DM-axis should also be taken into consideration when selecting a minimum slope threshold. In our parameter tuning trials we found that a slope threshold of 0.5 identified single pulses well regardless of the DMSpacing. The SNRRatio, a normalized ratio of the maximum SNR value to the SNR of the first point in a single pulse, proved to be a discerning attribute when ranking attributes by their information gain. 


\subsection{Single Pulse Classification with Machine Learning}

This section describes the machine learning techniques we used to classify single pulses. We tested the same six machine learning algorithms on single pulses that we used to classify DPGs in Chapter 5, as described in Section 2.2.2. We also used the same performance metrics described in Section 2.2.4 to evaluate our single pulse classifiers. In this section, we first discuss our choices for dealing with imbalance in the training data. Then, we introduce a new data-driven, automatic multiclass classification scheme for single pulses. Finally, we discuss how we identified the most relevant features to include in machine learning by using five different feature selection methods.

\subsubsection{Imbalance Considerations}

A data set is considered to be imbalanced when a large majority of its instances belong to only a few classes. For pulsar classification, the overwhelming majority of instances, for either single pulses or DPGs, are negative examples of RFI or noise. In the data set we used for single pulse classification, only $0.05 \%$ of the instances are from known pulsars. A supervised machine learning algorithm trained on imbalanced data will inevitably become biased toward the majority classes and more likely to miss the minority classes. This is especially true if there are very few samples of the minority classes.

While imbalance is still prevalent in our single pulse data set, examining SPEs rather than aggregated DPGs helped alleviate training bias by supplying more positive examples. Since a candidate plot can contain many single pulses from a single pulsar, we were able to identify ten times more positive training examples of single pulses from known pulsars than we could for DPGs [34]. This led us to expect better performance from single pulse classifiers trained on benchmark data sets with no imbalance treatment, which was confirmed by the results presented in Section 7.4 .

Rather than using all three imbalance treatments presented in Section 4.4.2, we chose to only test the SMOTE imbalance treatment. Based on the results presented in Sections 5.4.1 and 5.4.2, the performance of classifiers using the undersampling imbalance treatment suffered from not having a diverse enough population of negative training examples. On the 
other hand, classifiers using the oversampling imbalance treatment tended to overfit the positive training examples. Classifiers using SMOTE, however, were best able to accurately classify unseen data making them well-suited for pulsar searching.

It is important to note that care must be taken when applying any oversampling technique to avoid overfitting. Overfitting is when a classifier models its training data "too well", i.e., it learns to identify the intricacies of the training data exclusively to the detriment of its ability to classify new, unseen data. This can occur, for instance, when a classifier is trained and tested on the same data. Using the common method of $x$-fold cross validation (which divides a data set into $x$ bins, trains on $x-1$ of the bins, and tests on the holdout) on a data set that is heavily oversampled almost guarantees that the training and testing sets will overlap. To avoid this, we were careful to first divide our benchmark data sets into folds and then apply SMOTE only to the training folds, ensuring that our training and testing sets would be disjoint.

\subsubsection{Multiclass Classification}

In addition to being heavily imbalanced, pulsar classification in radio astronomy presents another challenge for supervised machine learning algorithms. Traditionally, pulsar classification is binary, i.e., a candidate plot either contains a pulsar, or it does not. However, candidate plots containing pulsars can appear drastically different, both to the eye and to a machine learning algorithm. Consider the plots labeled Pulsar and Very Bright Pulsar in Figure 4.3. The plots appear to be very different to the eye, but they are both technically pulsars. Similarly, a rule learner like JRip, for instance, would have difficulty developing a set of rules to classify both pulsars as a single class.

The differences in their appearances reflect their different physical properties, like distance from the observer and signal strength, as well as artifacts of data processing (such as the "clipping" of bright pulses mentioned in Section 4.4.1). Radio astronomers acknowledge some of these differences by labeling a few pulsar classes based on features of the source, e.g., millisecond pulsars are distinguished by their extremely short periods and RRATs are distinguished by the transience of their signals. Unfortunately, our extracted features for 
single pulses cannot determine periods and, while differences based on transience can be detected in aggregated DPG candidates (by features like AvgPulseCount), a single pulse relays no such information. Aiding our machine learning algorithms required a new approach to multiclass classification based on available features.

In Section 4.4.1, we performed multiclass classification by manually dividing the positive examples of DPGs in our benchmark into the four distinct classes shown in Figure 4.3. This visually-inspired multiclass classification scheme was more successful at detecting rare events with distinctive features (RRATs) than its binary counterpart (reported in Section 5.4.2). However, it had a significant drawback: the positive instances must be manually sorted by a human with a trained eye. This limitation is magnified by the task of single pulse classification, which yields many more positive examples than DPG classification.

Table 6.3: The features and thresholds used to perform Automatically Labeled Multiclass Classification.

\begin{tabular}{lcl}
\hline \hline Feature & Threshold & Label \\
\hline \multirow{2}{*}{ SNRPeakDM } & {$[0,100)$} & near \\
& {$[100,175)$} & mid \\
& {$[175, \infty)$} & far \\
AvgSNR & {$[0,8]$} & weak \\
& $(8, \infty)$ & strong \\
\hline \hline
\end{tabular}

To overcome this limitation and retain the benefit of learning from differences in groups of single pulses, we developed a new Automatically Labeled Multiclass (ALM) classification. Rather than using visual appearance, we categorized single pulses by two of their extracted features: SNRPeakDM, which is the DM value for the brightest SPE in the single pulse, and AvgSNR, which is the average brightness of all of the SPEs in the single pulse. This was accomplished by discretizing these numeric features and using their combinations to define class labels. We wrote code to automatically label known single pulses based on the thresholds given in Table 6.3. For comparison, we labeled each benchmark using five different class labeling schemes, as shown in Table 6.4. Each scheme is named by the number of classes it contains. Note that the scheme $4^{*}$ is identical to the classification scheme used by [34]. Also, to help determine if the new technique could still effectively classify rare instances, scheme 8 includes RRATs as a separate class. 
Table 6.4: The five different multiclass classification schemes tested.

\begin{tabular}{cl}
\hline \hline Scheme & Classes \\
\hline 2 & Binary: Non-pulsar, Pulsar \\
$4^{*}$ & Non-pulsar, Pulsar, Very Bright Pulsar, RRAT (34) \\
4 & Non-pulsar, Near, Mid, Far \\
7 & Non-pulsar, Near-Weak, Near-Strong, Mid-Weak, Mid-Strong, \\
& Far-Weak, Far-Strong \\
8 & Non-pulsar, Near-Weak, Near-Strong, Mid-Weak, Mid-Strong, \\
& Far-Weak, Far-Strong, RRAT \\
\hline \hline
\end{tabular}

The SNRPeakDM was chosen as a distinguishing feature for several reasons. The DM of a SPE is the integrated column density of free electrons along the line of sight between the observer and the SPE. In other words, if a long, narrow tube extended from the observer to the origin of the SPE, the DM would be proportional to the number of free electrons inside the tube. Theoretically, given the Galactic distribution of free electrons, the DM could provide the distance to the SPE and the SNRPeakDM the distance to the strongest SPE of a single pulse [81]. As a theoretical measure of distance, the SNRPeakDM makes sense as a possible categorical feature. Additionally, by using SNRPeakDM as a distinguishing feature we allow machine learning algorithms to take advantage of differences in other features that depend on DM, such as the DMSpacing. Finally, through examination of the distribution of known single pulses in the benchmark data, the SNRPeakDM was found to adequately group the known single pulses.

A key advantage of single pulse over DPG classification is its sensitivity to weaker signals, as measured by the SNR of a SPE. The AvgSNR represents the relative, average strength or 'brightness' of a single pulse, making it a potentially promising distinguishing feature. Furthermore, examination of known single pulses in the benchmark data revealed that several other metrics appeared to change proportionally to the AvgSNR, such as the number of pulses. 


\subsubsection{Feature Selection}

In Sections 4.3 and 6.2, we described the extraction of 22 characteristic features of a single pulse. In practice, not all features are relevant to classification. Irrelevant features may reduce classification performance and typically increase computational costs and memory usage of classifiers [14].

Table 6.5: The five different feature selection algorithms used.

\begin{tabular}{ll}
\hline \hline FS Algorithm & Type \\
\hline InfoGain (IG) & Entropy Measure \\
GainRatio (GR) & Entropy Measure \\
SymmetricalUncertainty (SU) & Entropy Measure \\
Correlation (Cor) & Linear Correlation \\
OneR (1R) & Machine Learning \\
\hline \hline
\end{tabular}

We independently applied five filtering feature selection techniques, listed in Table 6.5 and described in Section 2.2.5, to our data to explore what effect, if any, they had on performance. Each feature selection method provides a ranking for each feature which can be used to select only the top-ranked features. The implementations of each feature selection method are available through Weka [51]. To create each filter, we first divided each of our benchmarks into six folds. One fold was set aside and used only for feature selection, while the other five folds were used for training and testing our classifiers. Each feature selection technique was used to rank and select the ten most relevant features from the sixth fold. Before classification, the remaining five folds were filtered to contain only the ten features selected by the given feature selection technique.

Of the five feature selection techniques chosen, three depend on the measures of entropy described in Section 2.2.2, one relies on a measure of linear correlation, and one uses techniques from a simple machine learning algorithm. Each one provides a ranking for each feature which can be used to select only the top-ranked features. The implementations of each technique are available through Weka, with brief descriptions listed below. 


\subsection{Conclusions}

The work presented in this chapter provides methodological and algorithmic contributions. We extend the DPG identification and classification approach detailed in Chapter 4 to apply to the much finer-grained level of single pulses. To boost execution performance during the single pulse classification phase, we introduced a novel Automatically-Labeled Multiclass (ALM) classification technique which labels instances for supervised machine learning based on thresholds of key distinguishing features. We also used feature selection to reduce the dimensionality of our data and achieve execution performance improvements.

In Chapter 7, we evaluate our new methodology for single pulse candidate classification and identification by conducting a series of experiments using real-world radio astronomy data. 


\section{Chapter 7}

\section{Single Pulse Event Identification \& Classification: Evaluation}

\subsection{Introduction}

In this chapter, we present an application of the single pulse machine learning methodology described in Chapter 6 to real-world single pulse search data in order to classify transient radio signals.$^{1}$

Our research aims not only to classify single pulses, but also to explore several facets of machine learning and its application to real-world datasets. The research questions which guided our experimental exploration are divided into four broad categories:

1. the effectiveness of various machine learning algorithms,

2. the treatment of imbalanced data,

3. the comparative effectiveness of multiclass classification techniques, and

4. the efficiency of feature subset selection.

We measure success by the ability to classify instances both correctly and efficiently. Throughout this dissertation, we use the term classification performance to describe a classifier's ability to classify instances correctly and execution performance to describe efficiency

\footnotetext{
${ }^{1}$ The work in this chapter was originally published in [35].
} 
with respect to timeliness. With the looming prospect of peta- and even exa-scale data collection in radio astronomy (and many other fields), the execution performance of algorithms which process or mine data becomes increasingly important. To effectively classify very large data sets, algorithms must be scalable in addition to being time-efficient. In Chapters 8 and 9. we address Big Data concerns and ways to overcome them with scalable machine learning solutions.

The data analyzed for this chapter consisted of output from running PRESTO's single pulse searching software on data gathered from the long-term pulsar survey of the Galactic plane using the Arecibo L-band Feed Array (PALFA) [28]. PALFA is a seven-beam receiver operating at $1.4 \mathrm{GHz}$ with $0.3 \mathrm{GHz}$ bandwidth. The search began in 2004 and targeted low Galactic latitudes, $|b| \lesssim 5^{\circ}$, in the accessible longitude ranges, $32^{\circ} \lesssim \ell \lesssim 77^{\circ}$ and $168 \lesssim \ell \lesssim 214^{\circ}$

\subsection{Data Pre-Processing}

The output of a single pulse search performed with PRESTO are many long lists of SPEs, as described in Section 2.1.1. Our PALFA data set consists of the single pulse data for 6,638 sky positions, each observed with seven beams for a total of 46,466 observations made in the time span from March 2009 to May 2015. For each beam, there are up to 6,276 files, one for each DM tested from 0.0 to a maximum of 5,087.4. Each file contains data for the SPEs detected at that DM, and each SPE is described by its SNR, detection time, and downfact (a proxy for pulse width). As with the GBT drift-scan data described in Section 5.2, many of the almost 292 million files in the PALFA data set were empty or very small, leading to significant data bloat. Due to the minimum file allocation size, the 460 GB of data required 571 GB of storage space and the hundreds of millions of small files were difficult to transport and manipulate. We compiled the small files for each beam into one larger, standardized comma separated value (csv) format, reducing the size of the data by a factor of 1.58 (from $460 \mathrm{~GB}$ to $291 \mathrm{~GB}$ ) and the storage space required by a factor of 1.82 (from 571 GB to 313 GB). 


\subsection{Creating the Benchmark Data Sets}

The data used for the experiments in this work came from two sky surveys, GBT350Drift and PALFA (see Section2.1.2).

All of the classifiers presented in this chapter use supervised machine learning. Supervised machine learning depends on knowing the class values of all of the training data a priori, which required the creation of fully labeled benchmark data sets. To identify and label single pulses from known pulsars we followed a similar process as described for DPGs in Section 5.3 . i.e., using the ATNF Pulsar Catalog and RRATalog to search our data for single pulses in the immediate vicinity of all known pulsars and RRATs and then manually inspecting them for confirmation. From the 317 separate observations of 48 distinct pulsars in that data set, we were able to identify 5,204 single pulses. We combined these positive examples with 100,000 confirmed negative examples to create a single pulse benchmark for the GBT350Drift data.

To identify and label single pulses from known pulsars in the PALFA data set, we again used the ATNF Pulsar Catalog [87] and RRATalog ${ }^{2}$ to search our data for single pulses in the immediate vicinity of all known pulsars and RRATs and then manually inspected them for confirmation. This resulted in 3,170 single pulses from 98 known pulsars and RRATs, which were combined with 100,000 randomly selected and manually verified negative examples of single pulses from noise or RFI.

\subsection{Results}

For each of the two surveys studied, PALFA and GBT350Drift, we created a benchmark data set for each of our five multiclass labeling schemes. Each of these ten benchmarks were then divided into six folds, one for feature selection and the other five for training and testing classifiers. Then, five feature selection techniques were applied to the feature selection folds and used to filter out irrelevant features from the classification folds, also keeping an unfiltered set of benchmarks with no feature selection for a baseline comparison. We trained and tested the six supervised machine learning algorithms presented in Section 2.2.2, performing

\footnotetext{
${ }^{2}$ http://astro.phys.wvu.edu/rratalog
} 
five fold cross validation on each benchmark for a total of 1,800 individual classification trials. This process was repeated with another full set of benchmarks that were balanced by the SMOTE imbalance treatment for a grand total of 3,600 classification trials. The results are presented below, organized by the individual research questions explored. Only classification results for single pulses containing pulsars are reported throughout this section for two reasons: 1) We are mainly concerned with how single pulses containing pulsars are classified, and 2) the overwhelming majority of single pulses are correctly classified as non-pulsars and including them could falsely enhance the results.

\subsubsection{Machine Learning Algorithm Results}

The research question in this section explores which machine learning algorithms worked best for classifying single pulses in radio astronomy data. The results reported represent the 60 experiments performed without imbalance treatment, multiclass labeling, or feature selection.

\section{RQ 1: Which machine learning algorithm is best for single pulse classification?}

The results of the single pulse trials presented in Figure 7.1 are similar to those given in Section 5.4.1, which performed classification trials on DPGs. RandomForest achieved the highest scores in terms of classification performance (left plot), with the maximum scores for both surveys in Recall and F-Measure. On the other hand, the support vector machine, SMO, performed the worst. In terms of time efficiency (right plot), the artificial neural network (MPN) was again the slowest algorithm training on both surveys, while the tree learners (J48, PART) trained the fastest. All testing times were very low, with RandomForest taking slightly longer than the other algorithms. Overall, RandomForest appears to be the best algorithm for binary single pulse classification based on its superior Recall and F-Measure scores combined with its middle-of-the-road training and testing times. 

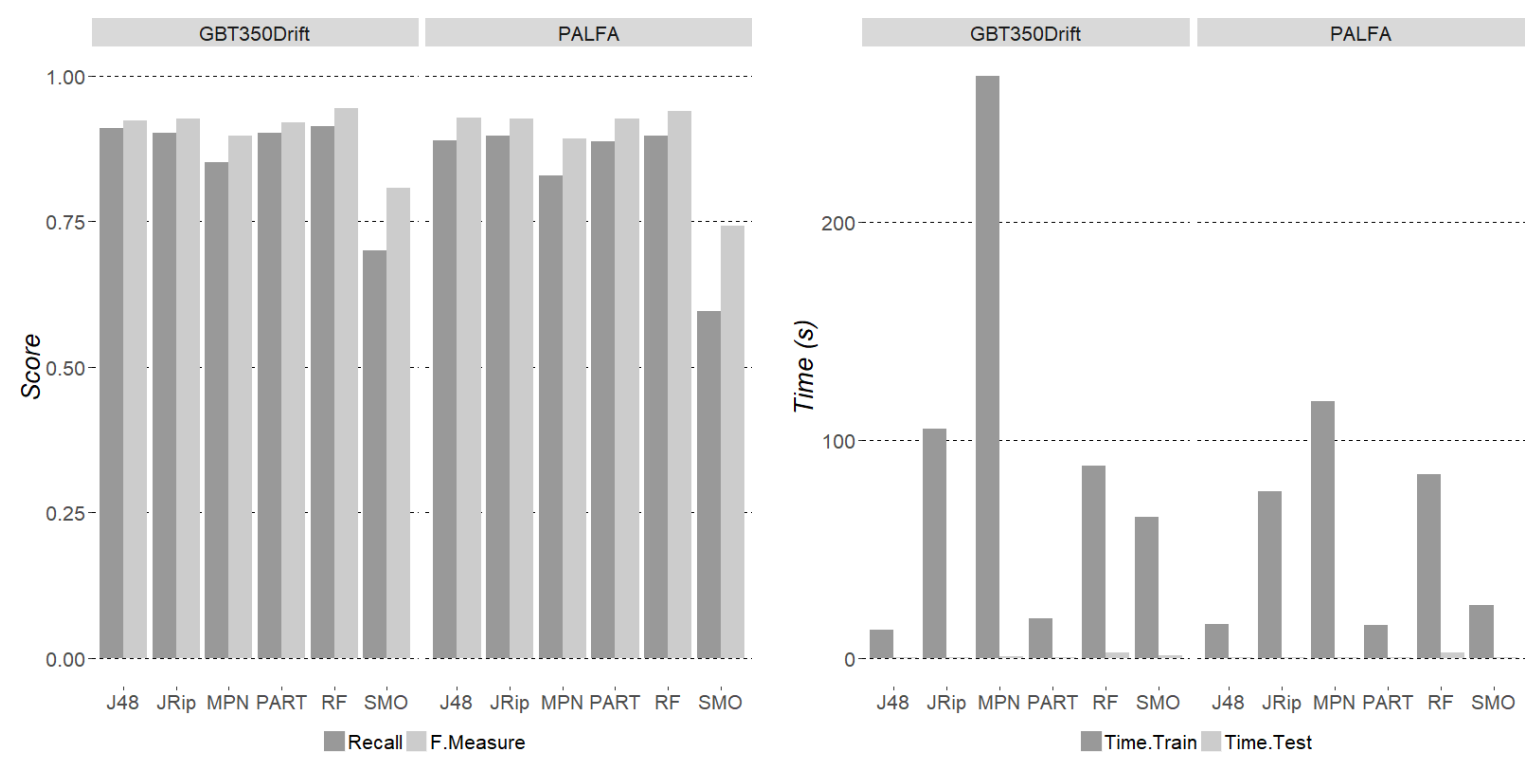

Figure 7.1: Bar charts showing the mean performance (left) and mean time efficiency (right) of six machine learning algorithms ( $x$-axis) organized by pulsar survey data.

\subsubsection{Imbalance Treatment Results}

The research question in this section explores how treating imbalance in the training data with SMOTE affects single pulse classification. Of the 3,600 experimental trials, only results of the 600 trials without feature selection are reported in this section.

\section{RQ 2: Does SMOTE improve single pulse classification?}

Figure 7.2 shows bar charts of the mean Recall and F-Measure scores (left) and training and testing times (right) for all classifiers subdivided by survey data and imbalance treatment.

The charts show similar trends in mean performance measures for both surveys, with RF performing the best and SMO the worst. The classifiers trained on data balanced by applying the SMOTE imbalance treatment consistently have higher Recalls and F-Measures than their counterparts trained with no imbalance treatment. As expected, both balanced and unbalanced classifiers' performances are generally better than those reported for DPGs in Section 5.4.1. We believe this is a consequence of having significantly more positive training examples for single pulses. However, the execution performance results indicate that the SMOTE imbalance treatment increases training times considerably. 

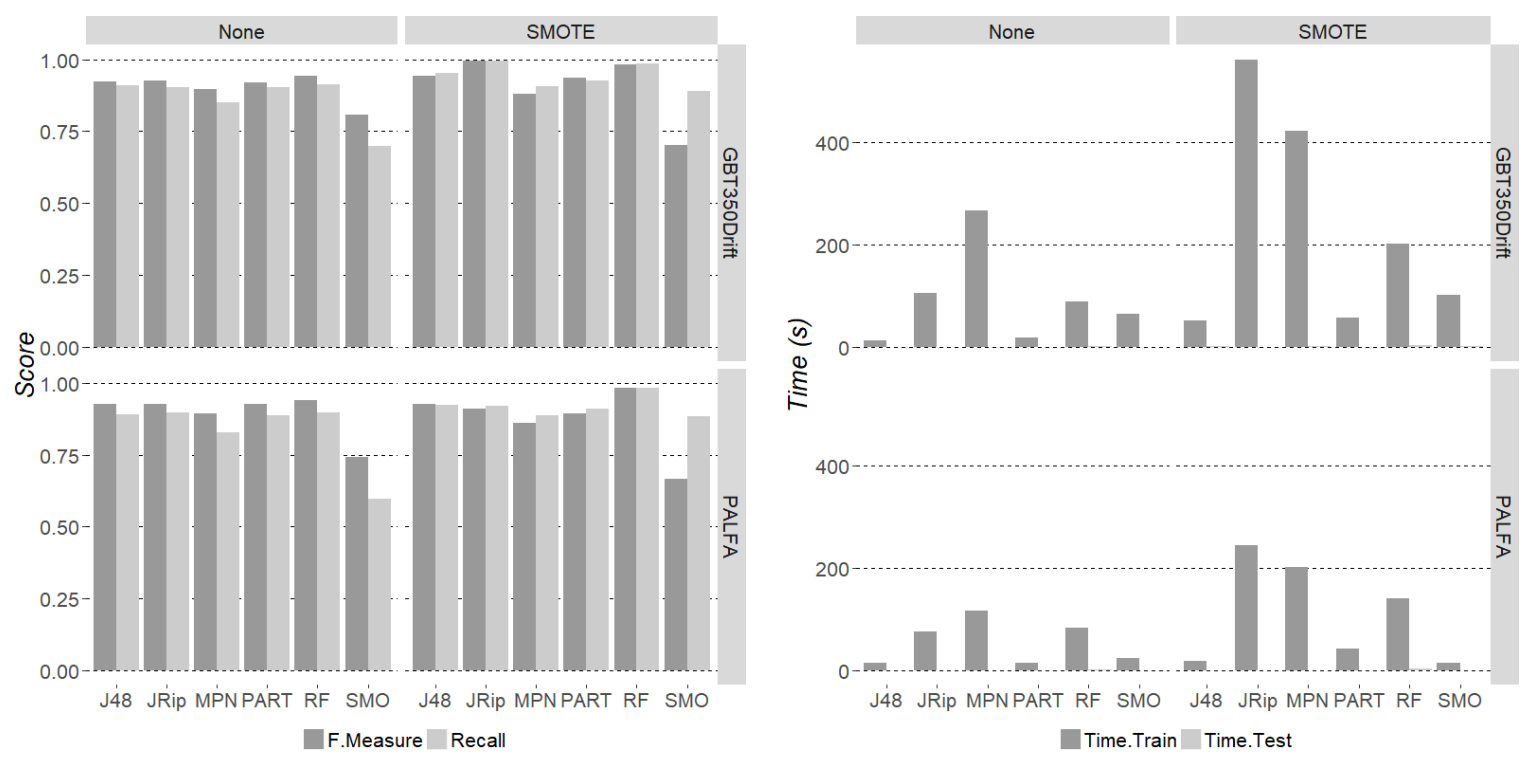

Figure 7.2: Two sets of bar charts showing the mean classification performance (left) and execution performance (right) of six machine learning algorithms. The charts are subdivided into rows by survey and columns by imbalance treatment, with "None" indicating no imbalance treatment.

\subsubsection{Multiclass Classification Results}

The research questions in this section explore the effects of different Automatically Labeled Multiclass (ALM) schemes on single pulse classification performance and execution performance. Of the 3,600 experimental trials, only results of the 600 trials without feature selection are reported in this section.

Multiclass labeling scheme $4^{*}$, which is the scheme successfully used to label DPGs described in Section 4.4.1, exhibited the poorest performance overall. Due to significant visual differences between single pulses and DPGs, it is not surprising that a visually-based multiclass labeling scheme for DPG classification is ineffective for single pulse classification. Scheme $4^{*}$ performs poorly throughout all experiments, and its results are omitted.

\section{RQ 3: Does ALM improve classification performance?}

Figure 7.3 shows boxplots of the Recall and F-Measure scores for all classifiers subdivided by ALM scheme (columns) and data set (rows). The plots show that performance results for ALM scheme 4 were comparable to those of binary classification for most algorithms. 


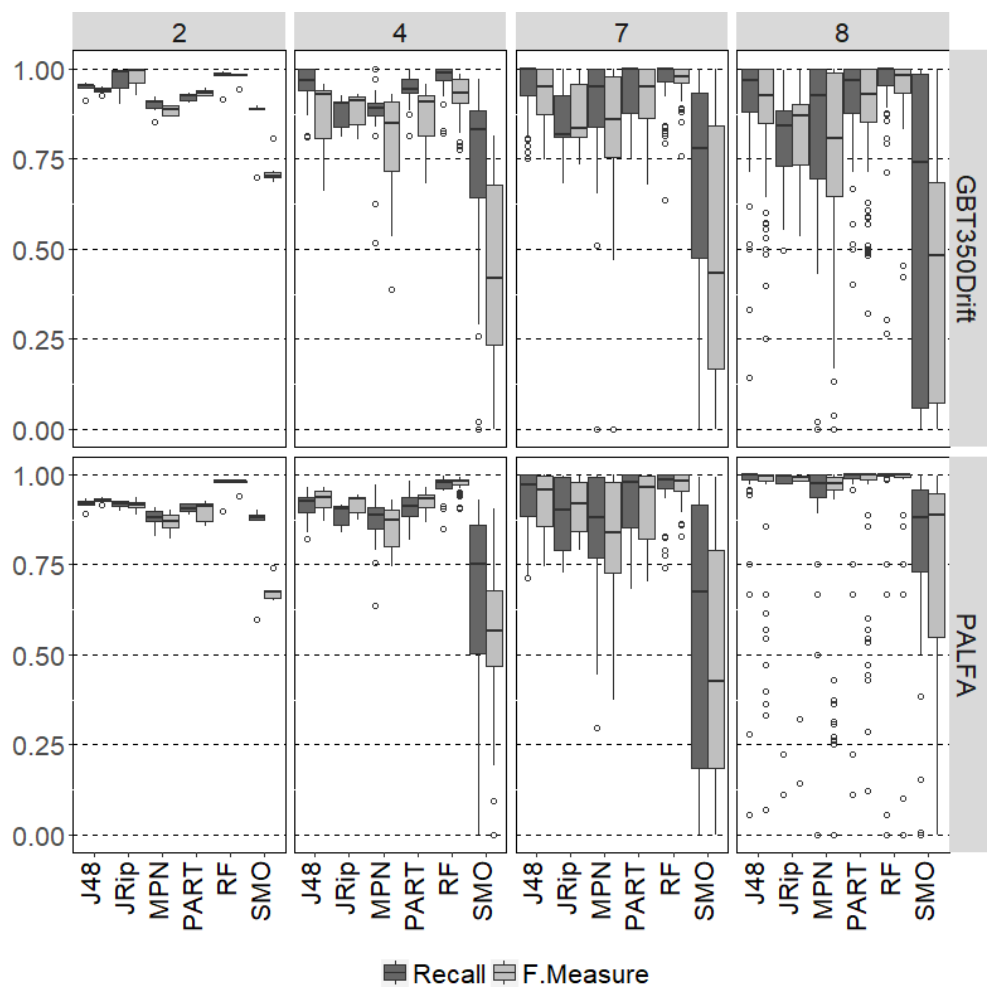

Figure 7.3: Boxplots of the Recall, F-Measure scores for all classifiers subdivided by ALM scheme (columns) and data set (rows).

Binary RF classifiers resulted in the highest median Recall and F-Measure scores with the smallest inter-quartile ranges (IQRs), except for the PALFA RF classifier using ALM scheme 8 , which performed the best overall.

\section{RQ 4: Does ALM improve classification performance for rare events?}

To explore this research question, we created a list of all positive instances of single pulses and which classifiers were able to correctly classify each of them. We used the list to determine which single pulses were mis-classified by the most classifiers. The twenty most mis-classified single pulses were missed by $90-99 \%$ of all classifiers. We found that ALM classifiers were more than twice as likely to correctly classify these problematic instances than binary classifiers. Furthermore, when expanding the analysis to include single pulses missed by 75 - $99 \%$ of classifiers, ALM classifiers were over three times more likely than binary classifiers to make correct classifications. This analysis also showed that RF classifiers were far better at classifying problematic single pulses, as they accounted for more correct 
classifications than all other classifiers with different algorithms combined.

\section{RQ 5: Does ALM improve execution performance?}

Figure 7.4 shows boxplots of training and testing times for all classifiers subdivided by ALM scheme (columns) and data set (rows). The boxplots show a noticeable reduction in median training times for J48, JRip, MPN, PART, and RF. Note that training times for SMO, which had the worst classification performance, had a high number of very large outliers as the number of classes increases (some are omitted from the plots to maintain scale), and a consistent increase in median training times. While RF classifiers exhibited the best overall classification performance, the simpler learners J48 and PART had the fastest training times. However, the long training times for RF classifiers were consistently reduced by the application of ALM. Overall, ALM scheme 8, which was automatically labeled by DM ranges and SNR strength, exhibited the fastest training times for RF (on average 56\% faster than binary RF classifiers). Testing times for all classifiers remain consistently low regardless of class labeling. RF, in particular, experiences the greatest performance increase with respect to time when testing multiclass scheme 8 (on average $48 \%$ faster). These results indicate that ALM improves the execution performance of classifiers while maintaining classification performance comparable to their binary counterparts.

Overall, ALM RF classifiers, on average, exhibited both Recall and F-Measure scores within $2 \%$ of their binary counterparts. Average total training times, however, were $47 \%$ less than binary RF classifiers. Due to these significant improvements in execution performance, combined with ALM RF's improved classification of difficult instances, ALM RF classifiers appear to be the best choice for single pulse classification.

\section{RQ 6: Does per class classification improve classifier performance?}

To answer this question, we ran additional trials over our benchmark sets and trained binary classifiers treating each multiclass labeling scheme as "1 vs. all". For example, for scheme 4, an additional binary RandomForest classifier was created for each of the three positive classes with each classifier treating its class as positive and all others as negative.

In Figures 10.1, 10.2, and 10.3 (see Appendix), the column headers show the multiclass 

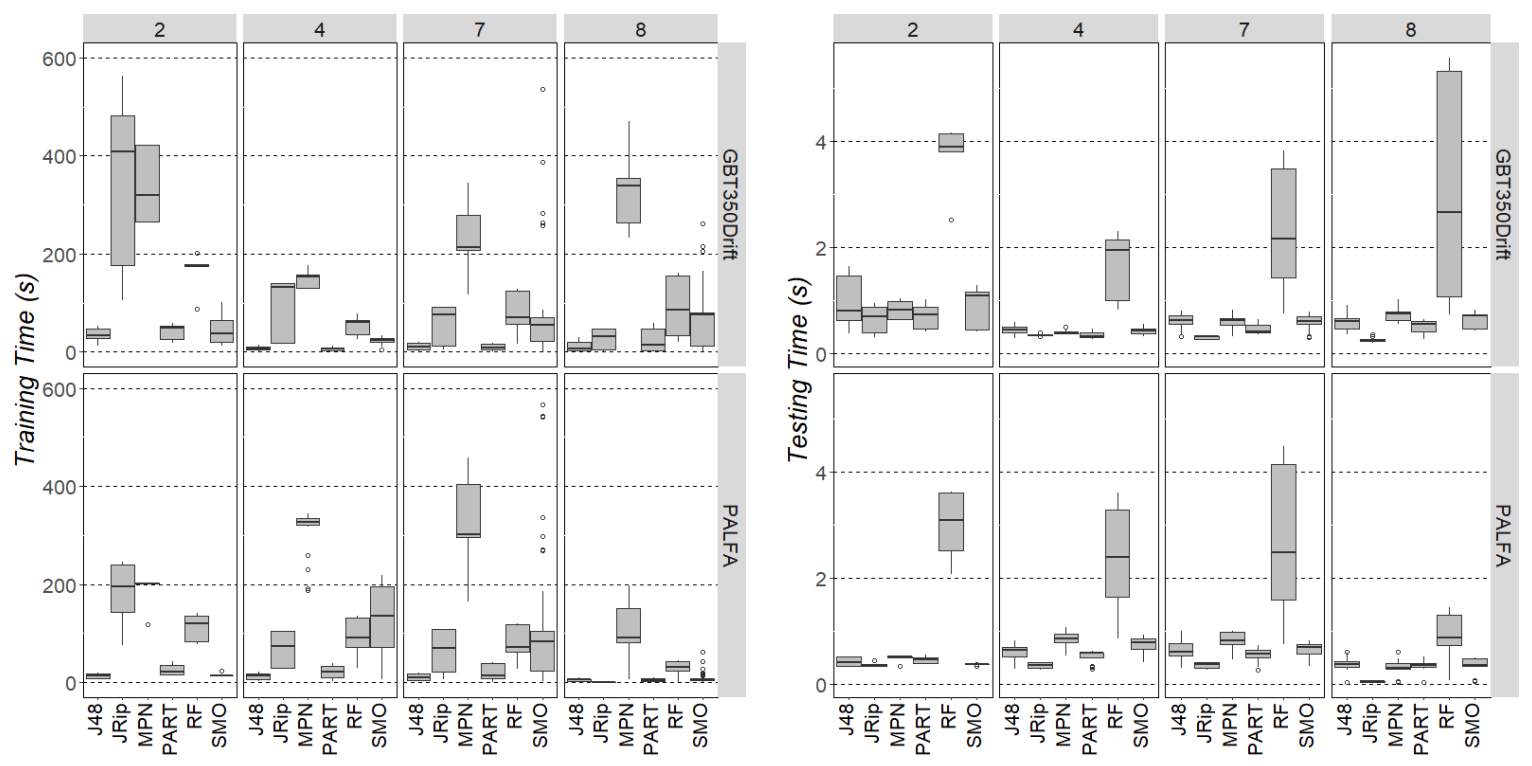

Figure 7.4: Boxplots of the training (left) and testing (right) times for all classifiers subdivided by ALM scheme (columns) and data set (rows).

classification scheme used (top) and the testing class (bottom). Each boxplot is split to show the training class on the $x$-axis. For per class classification to show a performance benefit, in Figure 10.1, the Recall and F-Measure scores on the left side of an individual boxplot (which represent training on a single class) would need to be higher than those on the right (which represent training on all classes). For example, no improvement is shown by the bottom right boxplot for the GBT350Drift survey (for the SMO classifier with multiclass labeling scheme 8 and tested on class 7), since higher Recall and F-Measure scores resulted from training on all classes than training only on class 7 .

Examination of Figure 10.1 shows no performance benefit from per class classification, as Recall and F-Measure scores for classifiers trained on individual classes are generally similar to or slightly worse then those trained on all classes. For RF classifiers, in particular, there is very little difference, which shows that per class classification does not degrade performance. However, Figures 10.2 and 10.3 show that per class classification can provide a performance boost to RF classifiers with respect to training and testing times. Per class classifiers have noticeably shorter training and testing times across the board than classifiers trained on all classes. 


\subsubsection{Feature Selection Results}

The research questions in this section explore the affects, if any, of feature selection on single pulse classification. This analysis includes results from all 3,600 classification experiments.

\section{RQ 7: Does feature selection improve single pulse classification?}

Figure 10.4 (see Appendix) shows boxplots of Recall and F-Measure scores from classification trials organized into columns by multiclass labeling scheme and rows by machine learning algorithm. The $x$-axis of each boxplot represents the five different feature selection techniques used in addition to the initial classifier with no feature selection. Figures 10.5 and 10.6 (see Appendix) are organized the same way to show boxplots of training and testing times, respectively.

The boxplots in Figure 10.4 show no overwhelming general patterns. In some cases, such as GBT350Drift's SMO with labeling schemes 7 and 8, the classifier with no feature selection outperforms all of the classifiers with feature selection. In other cases, such as classifiers from both surveys with JRip and labeling scheme 7, some feature selection techniques (InfoGain and GainRatio) boost classifier performance. For the RF classifiers, which exhibited the best overall classification performance, feature selection with IG, GR, and SU appeared to have no impact on Recall or F-Measure scores, while scores had larger IQRs and lower medians for Cor and 1R. Similarly, classification performance for MPN classifiers was not affected by IG and GR.

\section{RQ 8: Does feature selection improve the execution performance of single pulse classification?}

The execution performance with respect to training and testing times show a consistent improvement from certain feature selection techniques. Figures 7.5)(a) and 7.5(b) show boxplots of training times from classification trials organized into columns by ALM scheme and rows by data set, for only the RF and MPN classifiers. The $x$-axis of each boxplot shows the five different feature selection methods described in Section 6.3.3, as well as the initial 
classifier with no feature selection, annotated with "None" in Figure 7.5.

For multiclass $R F$ classifiers, certain feature selection techniques consistently improved execution performance with respect to training times. For the PALFA data set, ALM scheme 8 exhibited the most significant decrease with the least variability, while ALM scheme 4 performed best for the GBT data set. For multiclass $R F$ classifiers, the InfoGain feature selection technique resulted in consistently faster training times. Since Recall and F-Measure scores for multiclass $R F$ classifiers using Info Gain were comparable to those with no feature selection, we conclude that InfoGain, an entropy-based feature selection technique, decreased the execution time of single pulse $R F$ classifiers.

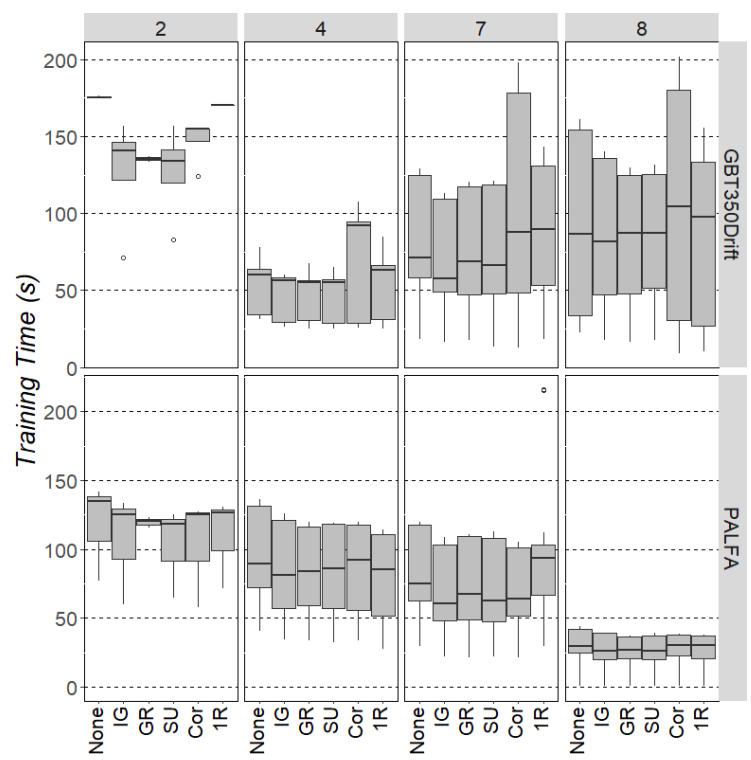

(a)

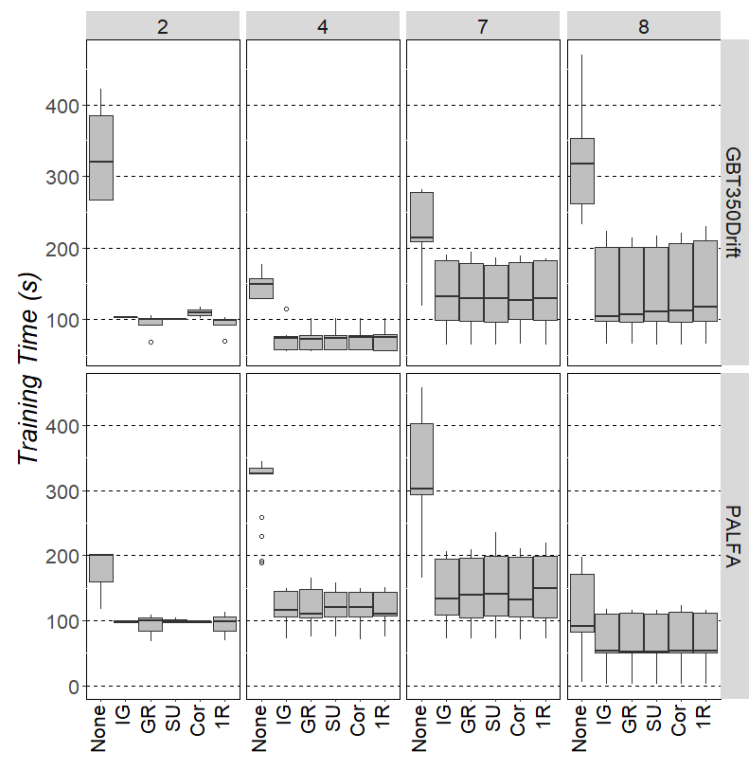

(b)

RF

Figure 7.5: Boxplots showing training times for RF (left) and MPN (right) classifiers. The $\mathrm{x}$-axis shows the feature selection method and the subdivisions show the ALM scheme for the GBT350Drift (top row) and PALFA (bottom row) data sets. Note that the scales are different.

Additionally, for all $M P N$ classifiers, all feature selection techniques resulted in reduced training times, in some cases (such as PALFA scheme 7) by up to 200 seconds. On average, training times for IG binary MPN classifiers were $64 \%$ lower than their counterparts without feature selection. The significantly longer training times of $M P N$ classifiers were their major drawback (note the difference in scale between Figures 7.5(a) and 7.5(b)). Figure 10.5(b) 
shows that feature selection could be an effective means of mitigating the poor execution performance of neural network classifiers.

\section{RQ 9: Are some features better than others for single pulse classification?}

To address this question, we compiled and examined a master list of feature rankings given to each feature by each feature selection technique. Figure 7.6 provides box plots of the rankings for each feature by the InfoGain feature selection technique and is subdivided by multiclass labeling scheme (columns) and survey (rows). Note that each feature selection technique selected and ranked only the top ten features for any particular data set, giving the $y$-axis in Figure 7.6 a scale from 1 to 10. In the boxplots, features with wide IQRs were inconsistently ranked from different data sets. The best features, then, are represented by compact IQRs appearing lower in the boxplots.

The first result of notice is that different features were selected as predictors for single pulses from different surveys. Specifically, InfoGain tended to select a wider variety of features to characterize single pulses from the GBT350Drift survey than it did for single pulses from the PALFA survey, especially for multiclass labeling scheme 8. Additionally, the IQRs for PALFA single pulse features are generally narrower than those of the GBT350Drift single pulses. This could be a result of the GBT350Drift survey containing over 2,000 more positive examples than the PALFA survey.

Another interesting result is that the rankings vary by multiclass labeling scheme. For instance, snrMax and snrLocalPeakHeight are very good predictors for scheme 2 in both surveys, but are ranked higher as the number of classes increases. This indicates that InfoGain is able to select different discerning features when single pulses are separated into like groups.

The most consistent, lower ranking predictors for all schemes are (in order from worst to less worse): SNRPeakDM, snrMaxFitChiSquare, pulseRank, startDM, and snrAvgIntensity. These features describe both physical characteristics (distance and brightness) and data characteristics (approximation of theoretical shape and brightness compared to other local peaks) of single pulses. These particular features are better predictors than others for single pulse classification. 


\subsection{Conclusions}

In this chapter, we experimentally evaluated the approach detailed in Chapter 6. We tested the performance of ALM and feature selection by performing classification trials using all combinations of six supervised machine learning algorithms, five ALM schemes, and six feature selection methods. We conducted the trials on two different benchmark datasets, enhancing the generalization of our conclusions. The results showed that while binary classification performances were very good, several multiclass schemes produced comparable classification results (within $2 \%$, on average) but with significant execution performance improvements. Overall, the ensemble tree RandomForest (RF) classifiers using ALM and the entropy-based InfoGain (IG) feature selection method achieved the best classification performance with average Recall and F-Measure scores of 0.96 and 0.95 , respectively. The ALM classifiers were also much better at correctly classifying instances that were often misclassified by binary classifiers. Further, the total execution performance of the classification improved on average by 54\%, (47\% from ALM and $7 \%$ from IG) when compared to RF classifiers without ALM and feature selection.

Our results from experimentally evaluating six machine learning algorithms and the SMOTE imbalance treatment on single pulse data contribute by reinforcement of the results we achieved in Chapter 4 for DPGs on a new real-world radio astronomy data set. Additionally, our feature selection results indicate that certain features based on theoretical and physical characteristics of single pulses are better predictors than others. This result will contribute to the streamlining of future work. 


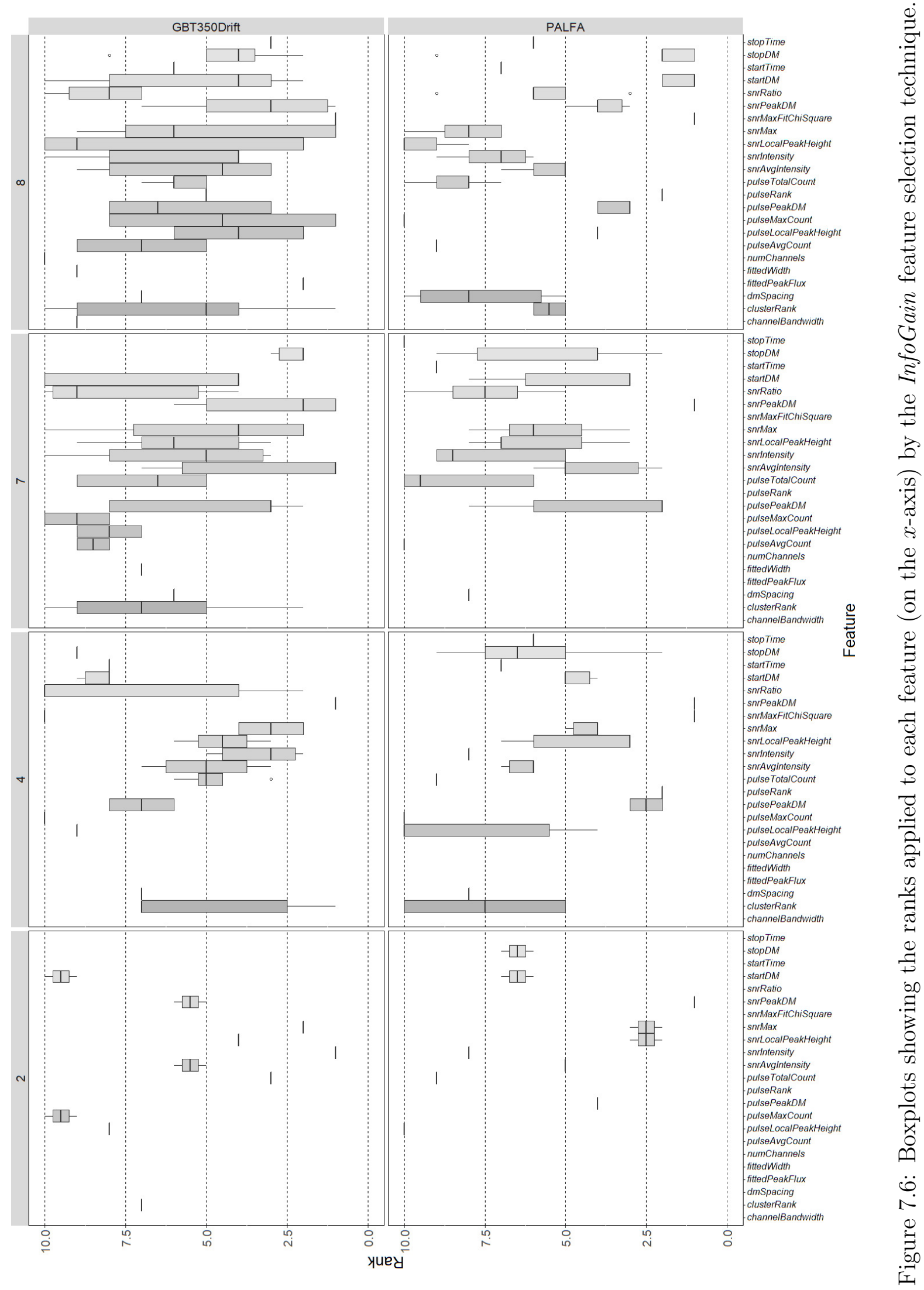




\section{Chapter 8}

\section{Distributed Single Pulse Event Identification: Methods \& Evaluation}

\subsection{Introduction}

With the advancement of data collection technologies, the commercial, government, and scientific sectors are flooded with more data than a single microprocessor could possibly process. In radio astronomy, as telescopes become larger and more sensitive, data collection rates are approaching peta- and exascale ranges [117]. The proposed Square Kilometer Array, which will combine 2,000 dishes and a million antennae over a collection area covering one million square meters, will be capable of collecting 160 terabytes of radio data a day [31]. For effective classification of very large data sets, algorithms must be time-efficient and scalable.

As our radio astronomy data sets grew in size and the granularity of our focus became finer, we found several key bottlenecks in the process of transient signal identification and classification. In this chapter, we present the parallelized solution we developed to overcome some of these bottlenecks along with empirical evidence of its successful application to two large real-world data sets from radio sky surveys, PALFA and GBT350Drift.1

\footnotetext{
${ }^{1}$ The work in this chapter was originally published in [35].
} 


\subsection{Distributed Single Pulse Identification}

The granularity of SPEs is much finer than that of DPGs. For example, the version of RAPID described in Section 4.2 considers only the maximum SNR for each DM and could only identify one DPG in the data represented in Figure 6.1. Our new distributed, single pulse version of RAPID identified 188 single pulses in the same data, including single pulse\#1 and single pulse\#2. Consequently, examining the data at this granularity is several orders of magnitude more processor intensive. For DPG identification, RAPID needs to run once for each observation. D-RAPID for single pulse identification, on the other hand, must run once for every cluster of SPEs in an observation. Since most observations contain several hundred to several thousand clusters, the modified algorithm must run several hundred to several thousand more times for each observation. Considering one data set used in our experiments encompasses almost 300 million observations, the workload is too much for a single computer to finish in a reasonable amount of time, even with multi-threaded programming. Fortunately, the problem of single pulse identification is "embarrassingly parallel", i.e., it may easily be divided into many smaller sub-problems which can then be solved simultaneously. The independence of the data combined with the uniform processing requirement makes searching for single pulses ideal for data parallelism on a distributed computing platform.

\subsubsection{Distributed Development Environment}

We designed scalability into our solution by parallelizing our application to run on a distributed YARN cluster with Apache Spark. YARN (Yet Another Resource Negotiator) is a resource management and job scheduling technology that makes two key improvements over the traditional Hadoop design. First, YARN decouples the programming model from the resource management infrastructure to allow programmers more flexibility for diverse coding applications. Second, YARN delegates many scheduling functions (e.g., task faulttolerance) to per-application components, effectively decentralizing the management of each job's control flow and thus improving scalability and efficiency [121]. Another advantage of a YARN architecture is its compatibility with Apache Spark. 


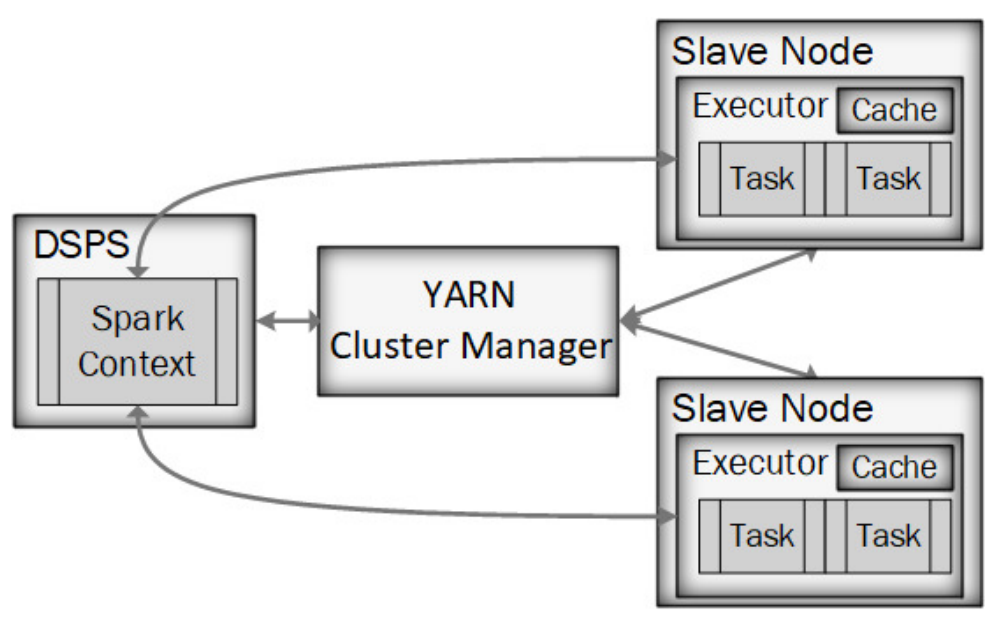

Figure 8.1: Control flow for a typical Spark job.

Spark is a fast and general distributed processing engine that has been shown to outperform the more traditional Hadoop MapReduce framework by ten times in iterative machine learning jobs [130]. Spark was designed for problems that need to reuse a working set of data across multiple parallel operations. To optimize such tasks, Spark introduced the resilient distributed data set (RDD), which is a collection of objects partitioned across a set of data nodes that can be rebuilt if a partition is lost. Spark achieves significant speed improvements by providing built-in functions to transform RDDs in memory, whereas MapReduce requires each intermediate step to be written to the HDFS. These qualities make a Spark on YARN cluster well-suited for the problem of efficiently identifying single pulses in massive data sets. As in Figure 8.1, the master node contains the partitioning structure for the distributed data in RAM and the tasks to be performed. The YARN cluster manager loads the data into distributed RAM according to the context-defined partitions, and maps the tasks to each data node. The data nodes then perform the sequence of transformations on its local data in RAM, returning the results to the master for aggregation.

\subsubsection{D-RAPID}

To take full advantage of distributed computing with Spark, we designed our data flow using RDDs. We wrote the original driver code behind RAPID in Java. For the redesign, we switched to the hybrid functional and object-oriented programming language Scala, which is 
the development language of choice for Spark applications [94]. Using Scala to develop our driver allowed for the seamless integration of our existing Java code and the subsequent single pulse modifications described in Section 8.2.3. In this paper, we annotate our distributed implementation of RAPID for single pulses in Scala as D-RAPID.

D-RAPID requires two input files to be loaded into the HDFS: a large data file containing all of the SPEs for an entire data set in csv format, and a smaller cluster file detailing each cluster to be searched (see Figure 6.2). The goal of D-RAPID is to search for single pulses only in the areas of the data file that coincide with the clusters listed in the cluster file. This poses a challenge for a distributed environment because the cluster information and the data belonging to it may not exist on the same data node. In the HDFS, a single file may be split into many chunks and replications and stored on several different data nodes. To solve this problem, D-RAPID organizes and joins the cluster and data files into a single RDD in memory to guarantee that each executor has all of the information it needs locally. This process is accomplished in three stages, as shown in Figure 8.2 .

Stage 1 and Stage 2 represent loading and preparing the input files. We first stripped the files of their header information. Then, in the Map to KVPRDD phase, we read each file from the HDFS and convert it into a Key-Value Pair RDD (KVPRDD), which can take advantage of the Spark's efficient built-in transformation functions. Since every instance in both the data and cluster files begins with the same descriptive information, i.e., the name of the data set, the mean Julian date (MJD) of the observation, its sky position, and the beam, we concatenated these descriptors to serve as the key for each instance. The value paired with each key is the remainder of the string containing the data for that instance, either SPE data or data describing a particular cluster. In Stage 3, we partition the data and cluster KVPRDDs and aggregate them to prepare for joining. D-RAPID uses Spark to optimize this process in two ways: uniform partitioning and key aggregation.

When joining two KVPRDDs, the key-value pairs must be shuffled and compared until they find their matches, which would result in an excessive amount of network traffic and overhead. Instead, we partition each KVPRDD in the exact same manner, so that the matching keys for each set are naturally co-located, eliminating unnecessary shuffling when performing the join operation[63]. In the Partition phase, D-RAPID uses a Spark HashPar- 


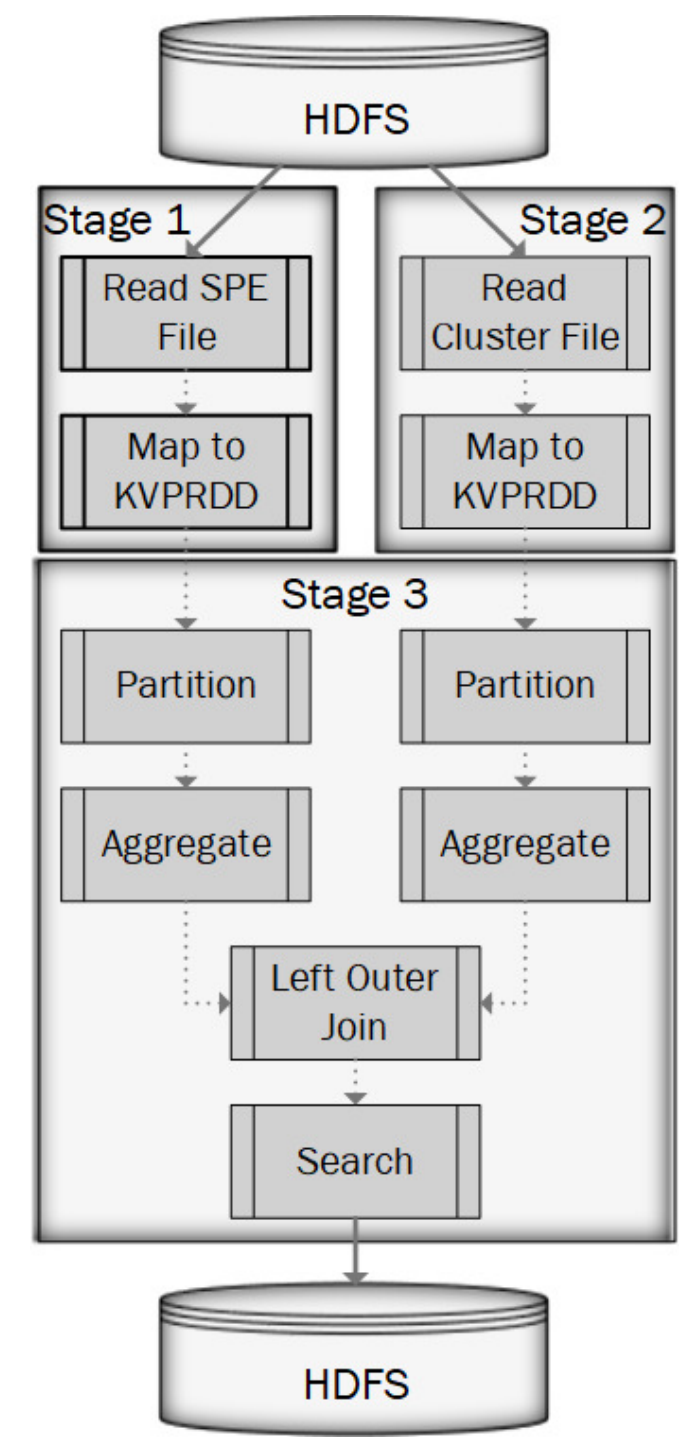

Figure 8.2: The stages of execution for D-RAPID to search a set of clusters for single pulses.

titioner to hash the keys and shuffle partitions with the same keys to the same executors from both data sets.

A join performed between two RDDs with many duplicate keys can significantly inflate the size of the joined data set [63]. An artifact of the csv format used for the data file is that there will be a very high number of repeated keys. In the Aggregate phase, we aggregate the cluster and data KVPRDDs by key so there will be less pairs when the expensive join operation is performed.

With the KVPRDDs optimized, we perform the join in the Left Outer Join phase. Generally speaking, a left outer join on two data sets will return a value for every entry of the 
first data set, even if there are no matching entries in the second data set (in which case a null value is returned) [104]. The end result of the left outer join of our data sets is a new KVPRDD with one KVP for each cluster together with all of the SPE data from the data file necessary to process it. This ensures that when we apply D-RAPID to the joined pairs in the Search phase, all necessary data will be local to the data node performing the operation. In the search phase, D-RAPID searches the SPE data from each cluster for single pulses. Finally, we write all identified single pulses back to the HDFS in separate files, which we later extract and from the HDFS and concatenate in preparation for the classification phase.

\subsubsection{Single Pulse Identification with D-RAPID}

Identifying single pulses in the DM vs. time space instead of aggregated DPGs in the SNR vs. DM space required several modifications to our previous searching algorithm. We developed Algorithm 1 to identify single pulses in the search phase of D-RAPID. The search algorithm uses recursion to divide the SPEs in a cluster into bins, performs a linear regression on the points in each bin to determine trends, and uses the trends to identify peaks in the data. For each bin, we consider the trend of the previous bin, $b_{n-1}$, which can be either decreasing, flat, or increasing. We next consider the slope of the current bin, $b_{n}$ and the state of a potential single pulse candidate, $S P$, to determine whether the SPEs in the current bin are "climbing" a single pulse, have reached the peak, or are "descending" a single pulse.

The search algorithm has several parameters that also required modification. The bin size parameter determines how many consecutive SPEs will be included in each regression calculation. For DPG identification in Section 4.2, we kept the bin size fixed at 25, a value that was chosen for its favorable experimental performance. However, a static bin size is not suitable when running on clusters, because they vary in size from several SPEs to thousands of SPEs. A static bin size of 25 will put all SPEs in small clusters into one bin, making it impossible for D-RAPID to identify a peak.

To accommodate this range of cluster sizes, in D-RAPID we assigned the bin size dynamically according to Equation 8.1, where $n$ is the number of SPEs in a cluster and $w$ is 


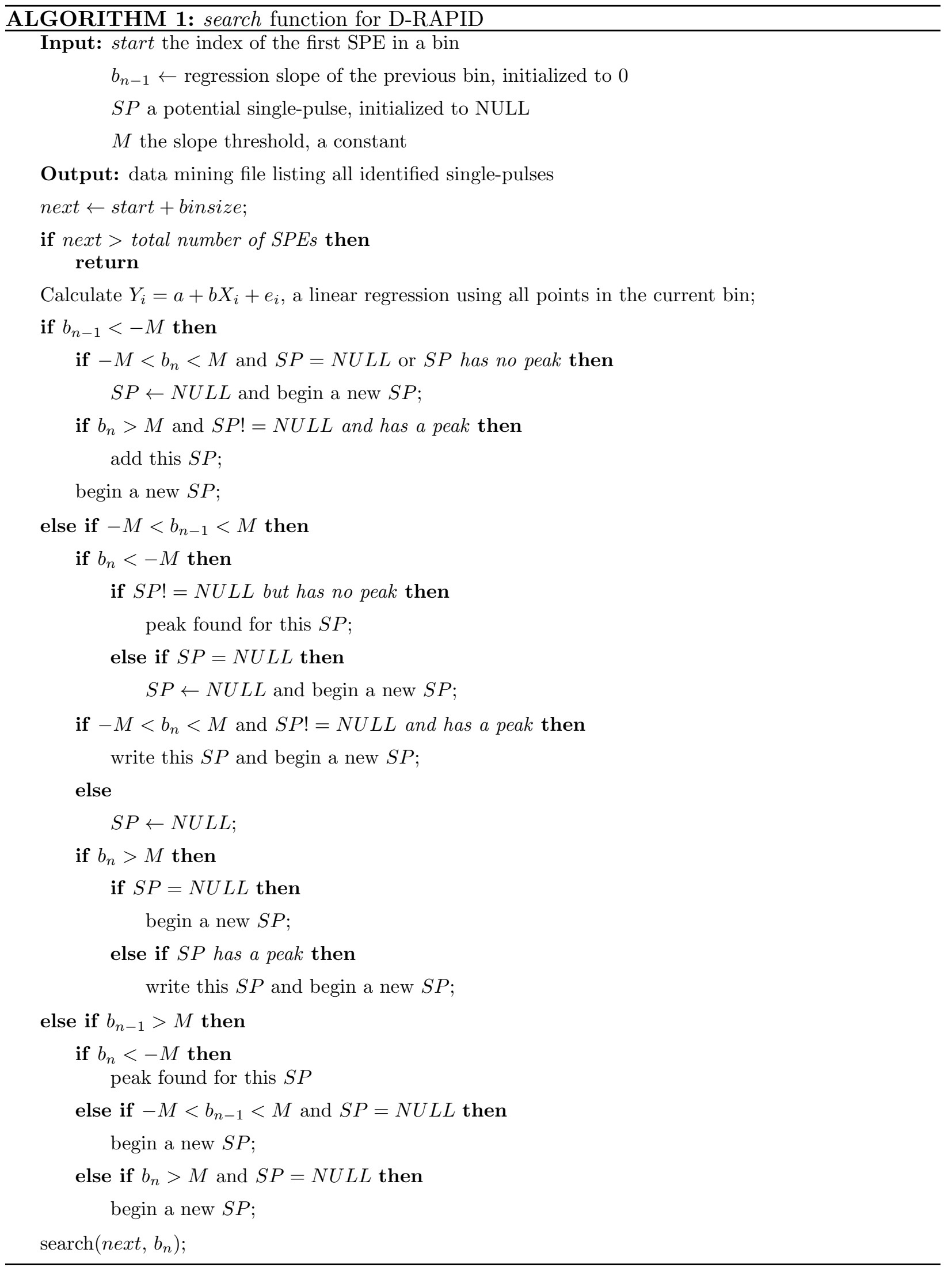


the weight, a new parameter which governs how quickly the bin size grows as the cluster size increases.

$$
\text { binsize }= \begin{cases}1, & \text { if } n<12 \\ \lfloor w \sqrt{n}\rfloor, & \text { otherwise }\end{cases}
$$

Using a bin size of one for small clusters simply "connects the dots" by considering the difference between two points. Varying the bin size by the square root of the cluster size ensures that the bin size increases quickly at first, and then levels out as cluster sizes become larger. The square root function exemplifies this behavior, but was found to increase too quickly for smaller clusters. To control the growth of the bin size, we introduced the weight parameter.

To tune the weight, $w$, and slope threshold, $M$, parameters, we chose several single pulses that are difficult to identify from known pulsars and used them for parameter tuning. The slope threshold provides a minimum slope requirement to distinguish between a linear regression line that is flat, or is increasing (or decreasing). In the parameter tuning experiment, we allowed the weight to vary from 0.75 to 1.75 and the slope threshold from 0.05 to 0.5 . The results showed that the combination of a weight of 0.75 and a slope threshold of 0.5 most efficiently identified problematic single pulses, and we used this parameter combination for the rest of the experiments.

\subsection{Results}

To demonstrate and evaluate the performance of our distributed approach to single pulse identification, we implemented our own distributed experimental environment and used it to empirically evaluate the performance of our scaled-up D-RAPID algorithm.

\subsubsection{Distributed Computing Environment}

The experimental environment consisted of 29 computers donated by Fairmont State University. Eight computers came equipped with a $3.2 \mathrm{GHz}$ quad-core Intel@ Core $^{\mathrm{TM}}$ i5$3470 \mathrm{CPU}$, a $250 \mathrm{~GB}$ hard drive, and $8 \mathrm{~GB}$ of RAM, and the remaining twenty machines 
had Intel@ Core $^{\mathrm{TM}_{2}}$ Duo E8600 CPUs clocked at $3.33 \mathrm{GHz}, 225 \mathrm{~GB}$ hard drives, and 4 GB of RAM. We installed 64 bit Ubuntu 18.04 on each machine and upgraded one of the i5 machines with an additional 24 GB RAM to serve as the master node. The other 28 computers were configured as data nodes. Overall, the distributed system provided 72 virtual cores, 140 GB of available RAM, and 4.2 TB of resilient data storage. We configured the distributed system to utilize the Hadoop YARN architecture, and managed it with the open source Apache cluster management software Ambari [122].

\subsubsection{D-RAPID Execution Performance Results}

We evaluated D-RAPID in our experimental environment on a 10.2 GB subset of the full PALFA SPE data set, which led to a 200 MB cluster file containing information for over 1.9 million clusters identified by the modified DBSCAN clustering algorithm presented by [97. In the distributed experimental environment, each executor was given two virtual processor cores and 2,560 MB of RAM so that the testing environment could support a maximum of 22 executors. Our custom partitioner assigned 32 partitions for each core, for a total of 896 partitions of the data. We recorded the total elapsed time to finish processing the test data in five separate trials, controlling the number of executors so that $1,5,10,15$, and 20 executors were allowed to operate in parallel.

For comparison, we also tested a multi-threaded version of RAPID for single pulse identification on the same subset of the PALFA data. The multi-threaded version ran on a single processor machine with 16 GB of RAM and an Intel@ Core ${ }^{\mathrm{TM}} \mathrm{i} 7-7800 \mathrm{~K}$ CPU overclocked to 4.5 GHz. We processed the test data five times, allowing the program to use $1,5,10,15$, and 20 threads to accomplish the task. Figure 8.3 provides the results.

RQ 1: Is D-RAPID scalable? The elapsed processing time for D-RAPID decreased as the number of executors increased, as Figure 8.3 shows. The knee of the curve for D-RAPID occurs when given five executors, and the elapsed time decreases asymptotically as the number of executors grows beyond five. These results indicate that D-RAPID can utilize Spark on a YARN cluster to dramatically reduce processing times for single pulse identification. While this is a good result, the significantly smaller decrease in elapsed processing time when 


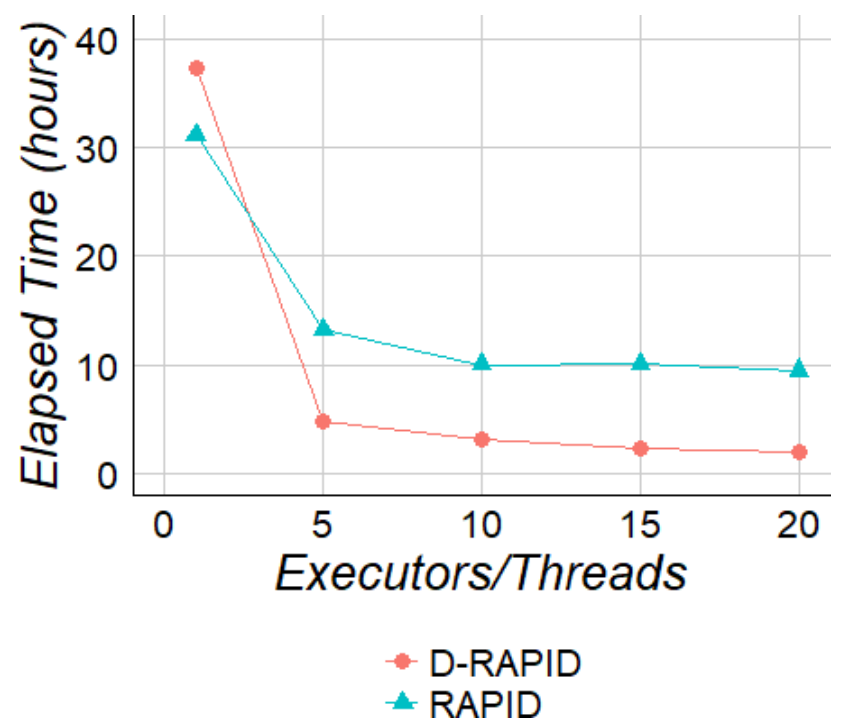

Figure 8.3: Performance evaluation of elapsed time to execute D-RAPID and a multithreaded version (RAPID) on a 10.2 GB test set with 5, 10, 15, and 20 executors or threads, respectively.

using more than five executors implies that other performance bottlenecks may exist.

One such bottleneck arises from the serial processing requirement for D-RAPID's search task, outlined in Algorithm 1, combined with the considerable size differences in various clusters. In the test data, individual clusters ranged in size from less than five SPEs to over 3,500 SPEs, with a median size of 19 SPEs. The sequential searching task took considerably longer for very large clusters. When examining the task distribution (for trials with more than one executor), some executors inevitably processed significantly less clusters due to these size differences.

The relatively small size of our test data set and the limitations of our experimental environment may have also impacted our performance results. We plan to further examine these potential bottlenecks in future work.

\section{RQ 2: Does D-RAPID outperform its multi-threaded implementation? D-RAPID} processed the same amount of test data in $22 \%$ to $37 \%$ of the time it took its multi-threaded counterpart in all trials, except when run with only one executor. A single executor, with only 2,560 MB of RAM, cannot fit the entire test data set into memory at once, which effectively eliminated the advantage of parallel processing with Spark, as portions of the 
RDDs must be frequently swapped out to disk to compensate for the lack of memory. This leads us to conclude that, as long as a YARN cluster has enough executors and memory to fit the entire data set into its distributed RAM, D-RAPID will consistently outperform the multi-threaded RAPID implementation by up to a factor of five.

D-RAPID also processed the entire PALFA data set described in Section 7.2 in 91.5 hours, a time that could be greatly reduced by adding more data nodes to the cluster. To contrast, a single machine with $16 \mathrm{~GB}$ of RAM and a 64 bit AMD Phenom ${ }^{\mathrm{TM}} \mathrm{II}$ X4 Black Edition quad-core processor took almost 580 hours to process the same data set using the single pulse version of RAPID.

\subsection{Conclusions}

Searching for single pulses in radio astronomy is a processor-intensive task that consists of identification and classification phases performed on very large data sets. The scalability and execution performance of pulsar identification and classification solutions must be considered to address the volume of data collected by the advanced radio telescopes of today and emerging technologies of the future.

In this chapter, we presented a software solution to improve the execution time of single pulse pulsar identification and classification. The main contribution of this work is the parallelization and refinement of the algorithm introduced in Chapter 4 along with an experimental evaluation of the results.

We improved the execution performance of the identification phase by developing Distributed Recursive Algorithm for Peak IDentification (D-RAPID), a Scala implementation of peak identification that achieves scalability by parallelizing the data on a Hadoop YARN distributed system for in-memory task processing with Apache Spark. We partitioned separate but linked data in memory to be co-located on data nodes. Experimental results showed that D-RAPID executed up to five times faster than a variant which achieved parallelism through multi-threaded execution.

Selected portions of the approach and results presented in Chapters 6, 7, and 8 were published in a highly selective conference on parallel processing [35]. 


\section{Chapter 9}

\section{Distributed Single Pulse Event Classification with Semi-Supervised Learning: Methods \& Evaluation}

\subsection{Introduction}

In Chapter 8, we motivated the desire for scalable machine learning methods in radio astronomy by the rapid data collection rates of modern instruments and the resulting extreme sizes of data sets. While Chapter 8 addressed the problem of identifying radio pulsar candidates in the data deluge, in this chapter we address the problem of radio pulsar candidate classification.

All previously described classification tasks in this dissertation used serial code running on single computers. Given that our largest classification file size is currently 9.5 MB (representing approximately 103,000 instances of single pulses from the PALFA data set), serialized classification code was not a problem. However, the full set of 5.85 million instances from PALFA is over 100 times larger (982.4 MB). Such large data sets are difficult, if not impossible, for serial classification algorithms to handle on a single workstation, even if the workstation has enough RAM to fit the entire data set in memory. Keeping in mind that these classification data sets will only get larger over time, the future of radio pulsar classification must utilize some form of stream or distributed computing platform to keep 
pace.

To compound the difficulty of dealing with very large data sets, labeling sufficient training instances for supervised classification is another problem caused by Big Data. All of the machine learning algorithms used for classification experiments in this dissertation thus far have utilized some form of supervised machine learning. For supervised machine learning to be effective, the labels for all training instances must be known a priori. To accommodate this necessity, we spent a significant amount of time creating fully labeled benchmarks by identifying and labeling known positive and negative instances in both the GBT and PALFA data sets, as described in Sections 5.3 and 7.3 . However, the instances we were able to label in the PALFA data set comprised only $1.7 \%$ of the total number of instances. This means that over $98 \%$ of the information available in the data is not used by our previous machine learning algorithms. To completely label a larger percentage of the instances would require manual inspection of each instance by a trained expert, with possible follow-up observations required for confirmation. Due to the prohibitive time, cost, and difficulty involved with assigning labels on such a large scale, supervised learning will never be able to utilize the majority of information available in the data.

Semi-supervised learning is an alternative to supervised machine learning that attempts to make use of unlabeled data as a potential source of knowledge [138]. There are many different types of semi-supervised machine learning algorithms, detailed in Section 2.2.3. The most relevant semi-supervised techniques to our work in this section are self-learning and co-training. The fundamental steps of self-learning and co-training are the same: 1) train a classifier on labeled data, 2) use that supervised classifier to label the unlabeled data, 3) add the best predictions from the unlabeled data back into the training set, and 4) repeat steps 1 through 3 until some stopping criteria are met. The difference between the algorithms occurs in steps 2 and 3. Self-training classifiers feed their best predictions back into their own training sets, and co-training classifiers enlist different base classifiers ${ }^{1}$ that feed predictions into each other's training sets [135].

In this chapter, we introduce CoDRIFt, a novel classification algorithm that is both

\footnotetext{
${ }^{1}$ The base classifiers can be the same classification algorithm trained on multiple, independent views of the data (such as different feature sets), or can be two different algorithms trained on the same feature set.
} 
distributed to overcome scalability issues and utilizes semi-supervised learning to leverage unlabeled data to inform the classification process. We demonstrate the utility of our new algorithm through experimental evaluation on two real-world radio astronomy data sets and provide a comparison to eleven other classifiers. The results showed that CoDRIFt models excel at classifying single pulses in data sets comprised of mostly (>95\%) non-pulsar signals. Furthermore, CoDRIFt models created with very limited sets of labeled data (as few as 22 labeled single pulses from pulsar sources) were able to achieve extremely high recalls (mean $=0.98)$. In comparison to the other algorithms trained with very few labeled instances, CoDRIFt models outperformed them all, with improvements in recall of $2.9 \%$ over the next closest classifier and an average improvement of almost $35 \%$ over all eleven classifiers. While developed to solve the radio pulsar classification problem, CoDRIFt is a machine learning algorithm that can be customized for different problem domains that deal with very large, imbalanced data sets, such as those found in financial fraud detection and cyber attack detection.

\subsection{CoDRIFt}

We propose a novel algorithm to create inductive Co-Training, Distributed, Random Incremental Forest (CoDRIFt) $)^{2}$ models for classification on distributed systems. The CoDRIFt algorithm presented in Algorithm 2 iteratively creates an inductive CoDRIFt model. The CoDrift model is a collection of trees that operates much like a RandomForest, with two key differences: 1) each tree is created by a different RandomForest model during training and selected as the "best tree", and 2) when making a prediction, a CoDRIFt model weights predictions for the positive class three times more than predictions for the negative class $3^{3}$ This form of weighted voting allows CoDRIFt models dealing with highly imbalanced data to better identify the minority class (i.e., achieve higher recall) at the expense of lower precision. This trade-off is acceptable for extremely rare instance classification in our application

\footnotetext{
${ }^{2}$ Available at https://github.com/tdevine1/cluster/tree/master/code/CoDRIFt

${ }^{3}$ We are assuming that the positive class is the minority class and the negative class is the majority. In our problem domain, the positive class represents SPEs originating from pulsar sources and the negative class represents SPEs originating from non-pulsar sources.
} 
area, as the cost of missing a rare instance (false negative) is much higher than that of having an extra false positive.

CoDRIFt models are constructed from three disjoint data sets. Two of the data sets must be labeled, one for training RandomForest models and one for evaluation of those models. The third, unlabeled data set is used to augment the labeled set for semi-supervised classification.

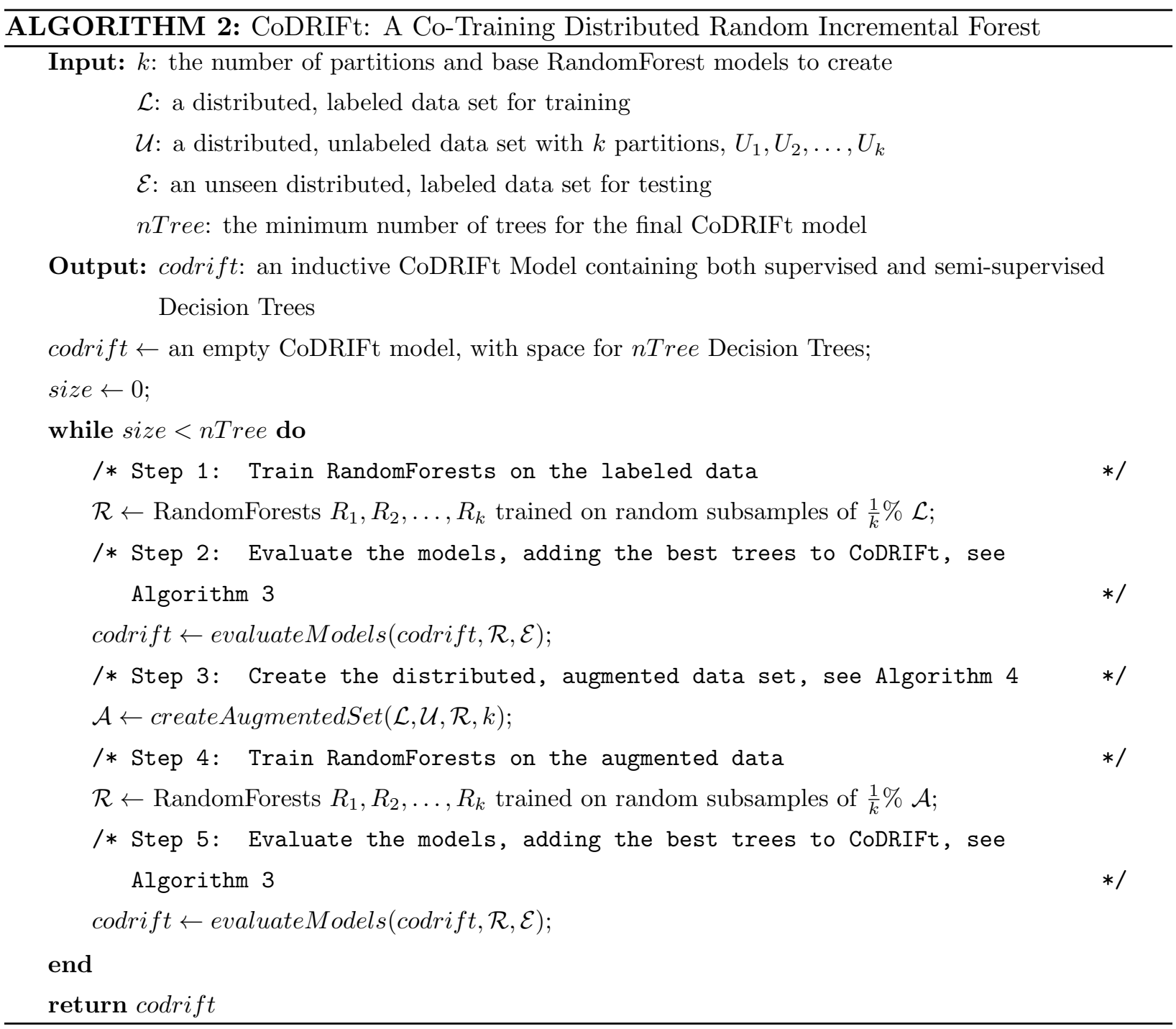

CoDRIFt operates in rounds, only stopping when the model has collected the userspecified number of trees. Each round consists of five steps. At the beginning of each round, in the first step, a new batch of $k$ (a user-defined value) RandomForest models are created from randomly selected samples of the labeled data set. The number of samples selected 
make up $\frac{1}{k} \%$ of the size of the data set. It is important to note that these subsamples are not stratified, and may not be representative of the distribution of the class value. This was done deliberately, so that some RandomForests may be trained on only negative examples in an imbalanced data set, as a classifier for imbalanced data must be very good at classifying negative examples with a high recall. In repeated trials during development, we noticed that CoDRIFt classifiers that only contained trees good at identifying positive examples routinely under-performed on imbalanced unseen data sets.

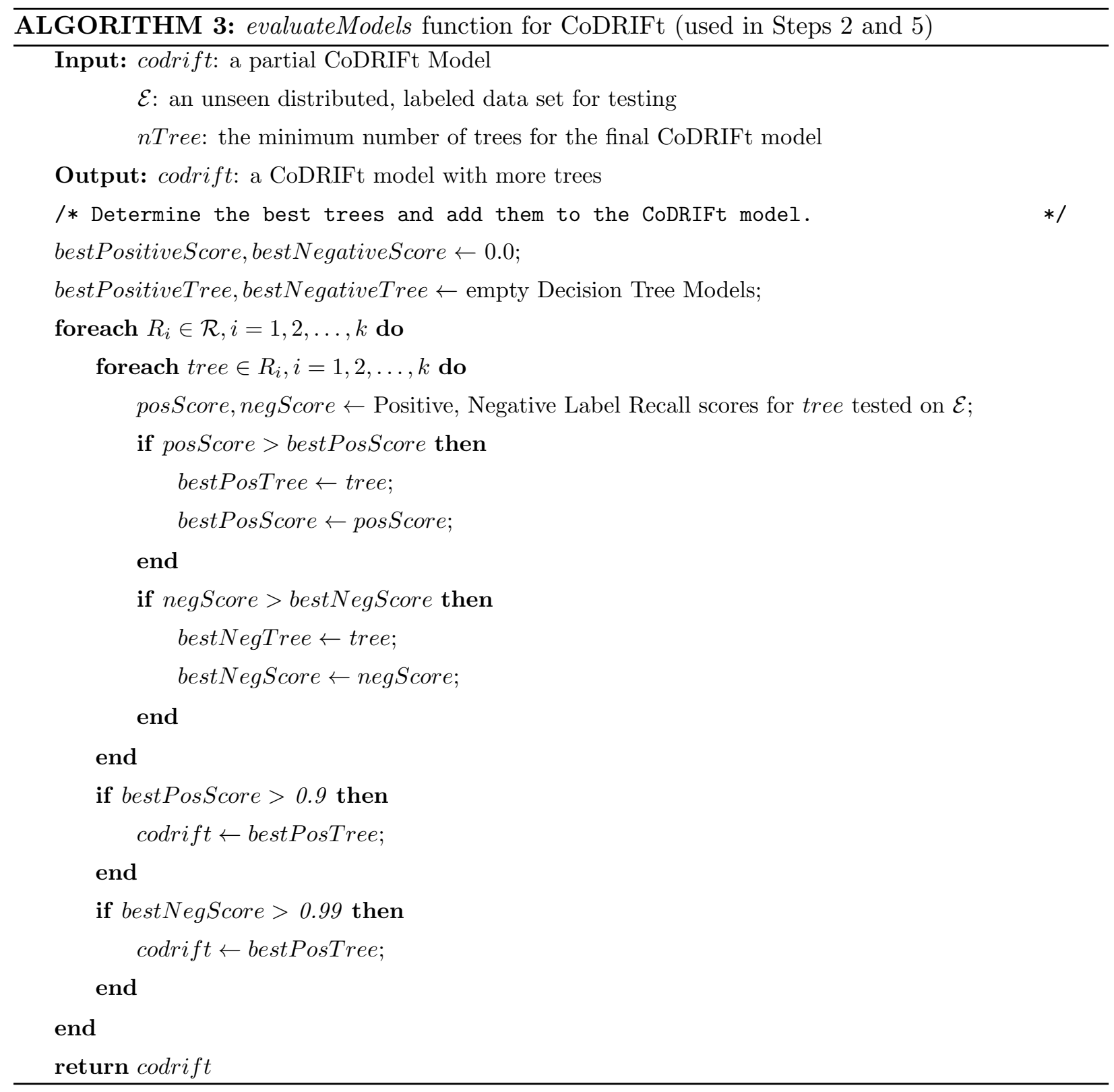


Once the models are selected, they are evaluated in step two. During evaluation, (see Algorithm 3), the best trees from each of the $k$ RandomForest models are determined by evaluating them individually on the unseen evaluation set. For each RandomForest model, two trees have the opportunity to be selected for inclusion in the CoDRIFt model, but no trees are guaranteed a spot. Each model determines its "best" two trees, one for classifying positive instances and one for classifying negative instances. In this version of CoDRIFt, recall scores were used to determine the best trees, but this could be altered to consider other performance metrics, as the situation dictates. We set inclusion criteria at recall $>0.99$ for negative trees (trees good at classifying negative examples) and recall $>0.9$ for positive trees (trees good at classifying positive examples). We chose these values to maintain diversity among the trees in the CoDRIFt model when there are many more negative than positive examples, since good negative trees in imbalanced data sets are a dime a dozen.

While steps one and two are supervised learning, the third and fourth steps of CoDRIFt involve semi-supervised learning. In the third step, shown in Algorithm 4, an augmented set is created that includes both the initial labeled set and all of the high-confidence predictions from the supervised RandomForests created in step one. To accomplish this, the unlabeled data is first divided into $k$ partitions. Then, in parallel, a random supervised RandomForest model is chosen to predict labels for the unlabeled data in each partition. If a prediction has a confidence $\geq 0.95$, that instance is added to the augmented set with its new label. In the fourth step, a new batch of $k$ semi-supervised RandomForest models are trained on the augmented data set in the same manner as in step one. Finally, in the fifth step, the semi-supervised RandomForest models are evaluated on the unseen evaluation set, and the best trees are included in the CoDRIFt model, following the methodology of step two.

The end result is an inductive, semi-supervised CoDRIFt model containing the best supervised and semi-supervised trees from many different supervised and semi-supervised RandomForest models. The closest algorithm in the literature to CoDRIFt is the selflearning algorithm presented in [119]. The main novel aspects of CoDRIFt that set it apart from the other algorithms in the literature are:

1. We create many RandomForest models and keep only the best decision trees from each 


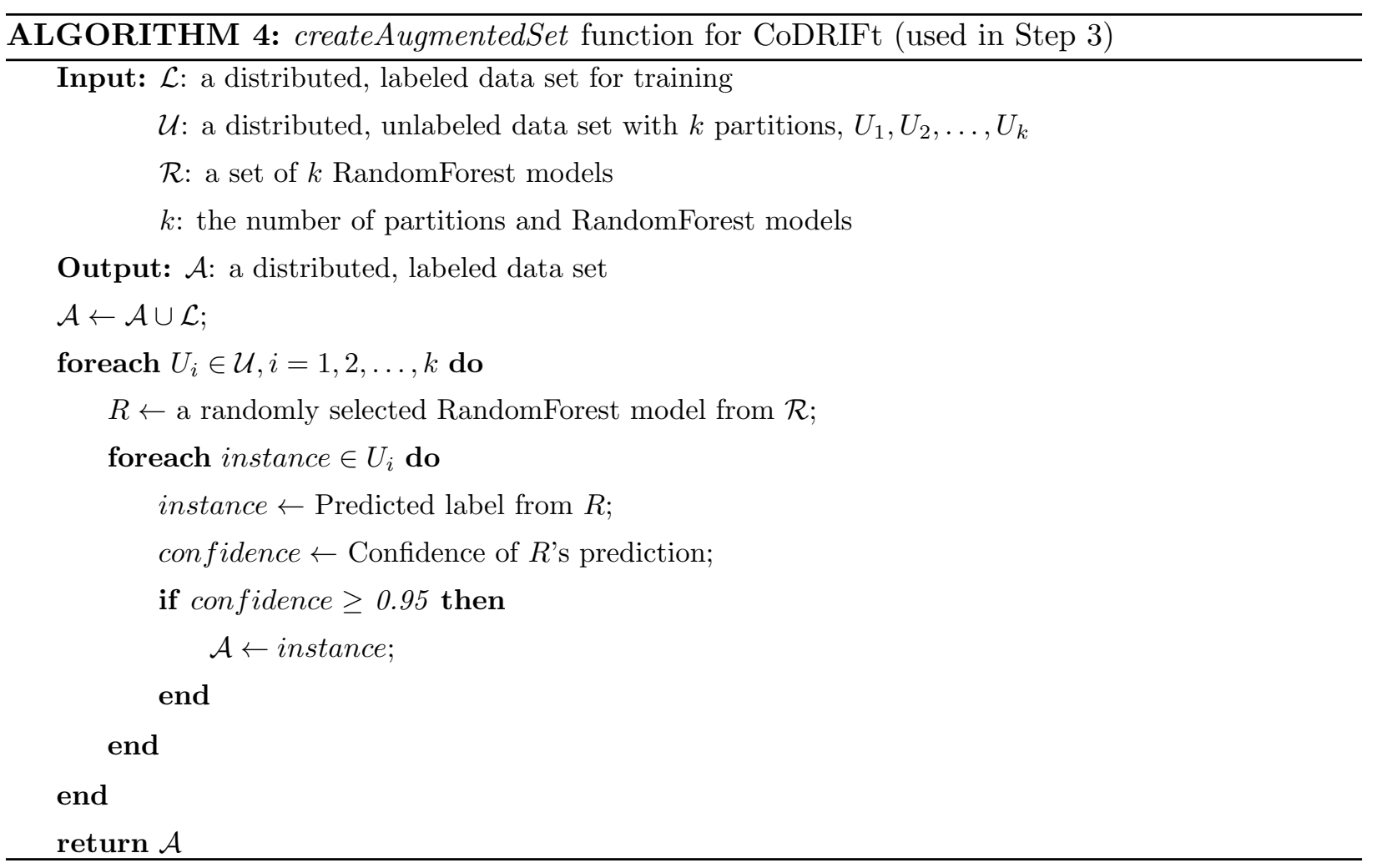

model. We place an emphasis on composing an ensemble of stronger learners, rather than training learners on larger data sets with more labels.

2. Our final model contains a mix of supervised trees (trained only on labeled data) and semi-supervised trees (trained on data augmented with labels from other RandomForest models).

3. We optimize our model to achieve higher recalls by selecting trees with high recalls on unseen data.

4. We use weighted voting when making predictions, as in [25], but count positive predictions three times heavier than negative predictions instead of weighting each individual tree. This helps our algorithm perform well in imbalanced data sets.

5. Each iteration, the unlabeled set resets, allowing instances to be labeled multiple times by different trees in different iterations. This means unlabeled instances will not necessarily be labeled the same every time, and new trees could be trained on the differently labeled data. 
The CoDRIFt model is general enough to be used as a distributed, semi-supervised classifier in any large data set where the cost of labeling is prohibitive and is designed to work especially well in problem domains that deal with extremely imbalanced data, such as cybercrime or credit card fraud.

\subsection{Algorithms Used for Comparison}

In order to provide a thorough, comparative evaluation of CoDRIFt's performance, we tested the following eleven algorithms for semi-supervised learning and distributed classification: Collective Classification, SupervisedMLRF, and SelfLearningMLRF. Collective Classification $\rrbracket^{4}$ is a Java implementation of nine serialized semi-supervised classification algorithms for the Weka suite of data mining software [51]. SupervisedMLRF is a distributed Scald 5 implementation of a RandomForest algorithm. Scala is a hybrid programming language that combines object-oriented and functional programming that was designed for high performance computing on distributed systems. SupervisedMLRF is publicly available through Apache Spark's machine learning library (MLlib)6, Finally, SelfLearningMLRF is our distributed Scala implementation of a general self-learning algorithm that uses a modified version of SupervisedMLRF as its base classifier [119]. In this section, we provide descriptions of these algorithms.

\subsubsection{Collective Classification}

Collective Classification is the only available semi-supervised learning package for Weka. Collective Classification, which found its start with networking applications, refers to a group of nine different machine learning algorithms that attempt to take advantage of the relationships between instances in partially labeled collections. More specifically, Collective Classifiers make classification decisions based on correlations between: 1) the label of an instance and that instance's observed attributes, 2) the label of an instance and the observed

\footnotetext{
${ }^{4}$ Available at https://github.com/fracpete/collective-classification-weka-package

${ }^{5}$ https://www.scala-lang.org/

${ }^{6}$ https://spark.apache.org/mllib/
} 
attributes (and labels) of the instances nearby, and 3) the label of an instance and the unobserved labels of instances nearby [114].

For our comparison with CoDRIFt, we performed classification with the following Collective Classifiers:

- SimpleCollective is a self-training algorithm that randomly assigns labels to the unlabeled instances in the training set, and then iteratively trains a user-specified classifier on all of the data and uses the predictions to improve the randomly assigned labels.

- AdvancedCollective operates similarly to SimpleCollective, but avoids overfitting by splitting the randomly labeled data into two groups. One group is used for training together with the labeled instances and the other group for testing (and vice versa).

- CollectiveEM is similar to SimpleCollective, but duplicates the unlabeled data to make two training sets. Each unlabeled instance is randomly made positive in one training set and negative in the other. The base classifier is also duplicated and used to iteratively improve labels, with the assignment of labels depending on the mean of the confidences of the two classifiers to avoid oscillating behavior.

- CollectiveForest is a self-training, tree-based classifier that divides the test set into equally dense folds with Weka's RandomTree (the basic trees built with randomly selected features that comprise a RandomForest) algorithm, then uses the training set to predict the labels for instances in the test set. The labeled instances with the highest confidence are then added into the training set. This process is repeated until all labels are predicted with high confidence [111].

- CollectiveWoods and CollectiveTree - CollectiveWoods operates similarly to CollectiveForest, but uses the CollectiveTree algorithm as a base classifier instead of RandomTree. CollectiveTrees are different from RandomTrees by favoring attribute split points that evenly divide the data in two. Additionally, a CollectiveTree will stop growing if it only covers training instances, if its leaves contain only test instances, or if a leaf contains only training instances from one class. 
- TwoStageCollective uses a form of co-training with two or more user-given classifiers. The first classifier is built using the labeled data and makes predictions for the unlabeled data. The second classifier is built on all of the data and used to enhance the predictions of the first. Additional user-specified classifiers are used alternately to continue the iterative process 99 .

- YATSI is "Yet Another Two Stage Idea" that uses co-training with a user-specified classifier together with a K-Nearest Neighbors (KNN) classifier. The first classifier is used to train a model on the labeled data and "pre-label" the unlabeled data. The pre-labels are then reevaluated by considering the labels of the closest $k$ instances, keeping the label with the highest confidence [38].

- Chopper is an implementation of the traditional co-training classifier specified in Section 2.2 .3 .

\subsubsection{SupervisedMLRF}

SupervisedMLRF is a supervised RandomForest algorithm implemented in Scala as part of Apache Spark's MLlib package. Since RandomForests (as described in Section 2.2.2) train sets of decision trees independently, they are naturally parallelizable. RandomForests were also shown to be excellent radio pulsar candidate classifiers in all of our previous experiments, making them an easy choice for our distributed classification trials. SupervisedMLRF uses data-parallel optimizations to improve the performance of the algorithm, which operates by subsampling the training data for the construction of each tree (bootstrapping) and also considering random subsets of the features for determining the nodes of each tree. The SupervisedMLRF models we created throughout all of our experiments used Gini Gain (see Section 2.2.2 to determine split points for individual decision trees. They created binary decision trees with a maximum depth of four and a varying number of trees depending on our experiment.

The SupervisedMLRF implementation, however, has several drawbacks as noted by the authors in 25]. First, when determining the best splitting point for continuous features, SupervisedMLRF samples each partition of the data set to reduce the data transmission 
operations. This method could reduce the accuracy of the models created. Additionally, SupervisedMLRF partitions Resilient Distributed Data sets (RDDs) horizontally, (by instance, not by feature). This causes some overhead global data communication when computing splitting points for nodes.

Spark's MLlib has several limitations we had to work around to implement and evaluate SupervisedMLRF-based algorithms. First, stratified random sampling is not available outof-the-box with our Spark MLlib version (2.3.2), so we implemented our own version in Scala to ensure that each time we sampled a data set, the sample would contain a representative distribution of class values. Additionally, due to the way that RDDs are stored and managed in distributed RAM, we were not able to split them into folds for any type of cross validation and had to rely on subsampling with repetition to validate our experimental results.

\subsubsection{SelfLearningMLRF}

SelfLearningMLRF is based on the general self-training algorithm presented in [119] (see Algorithm 5). Following their observation that basic decision trees were unreliable as base classifiers in self-training due to unreliable predictions, we used a modified version of SupervisedMLRF as our distributed base classifier in our self-learning implementation. The original SupervisedMLRF did not keep track of the confidence of predictions it made. It has been noted that high-confidence predictions are necessary for self-training to improve when labeling unlabeled instances to add into the training set [119]. To allow for this in our implementation, we altered the predictByVoting method to return a confidence value along with its predicted label for a given instance. We calculated the confidence as the number of votes for the predicted label divided by the total number of trees in the model. This allowed us to specify a confidence threshold, $t$, for our implementation.

As shown in Algorithm 5, SelfLearningMLRF starts with a base classifier, Spark's SupervisedMLRF, and trains it on the fully labeled training set. The trained forest is then used to predict a label for each instance in the unlabeled set. If the classifier is very confident in its prediction (we chose $t=0.95$ ), then that instance is removed from the unlabeled set and added to the labeled set for training future iterations. The process then repeats itera- 


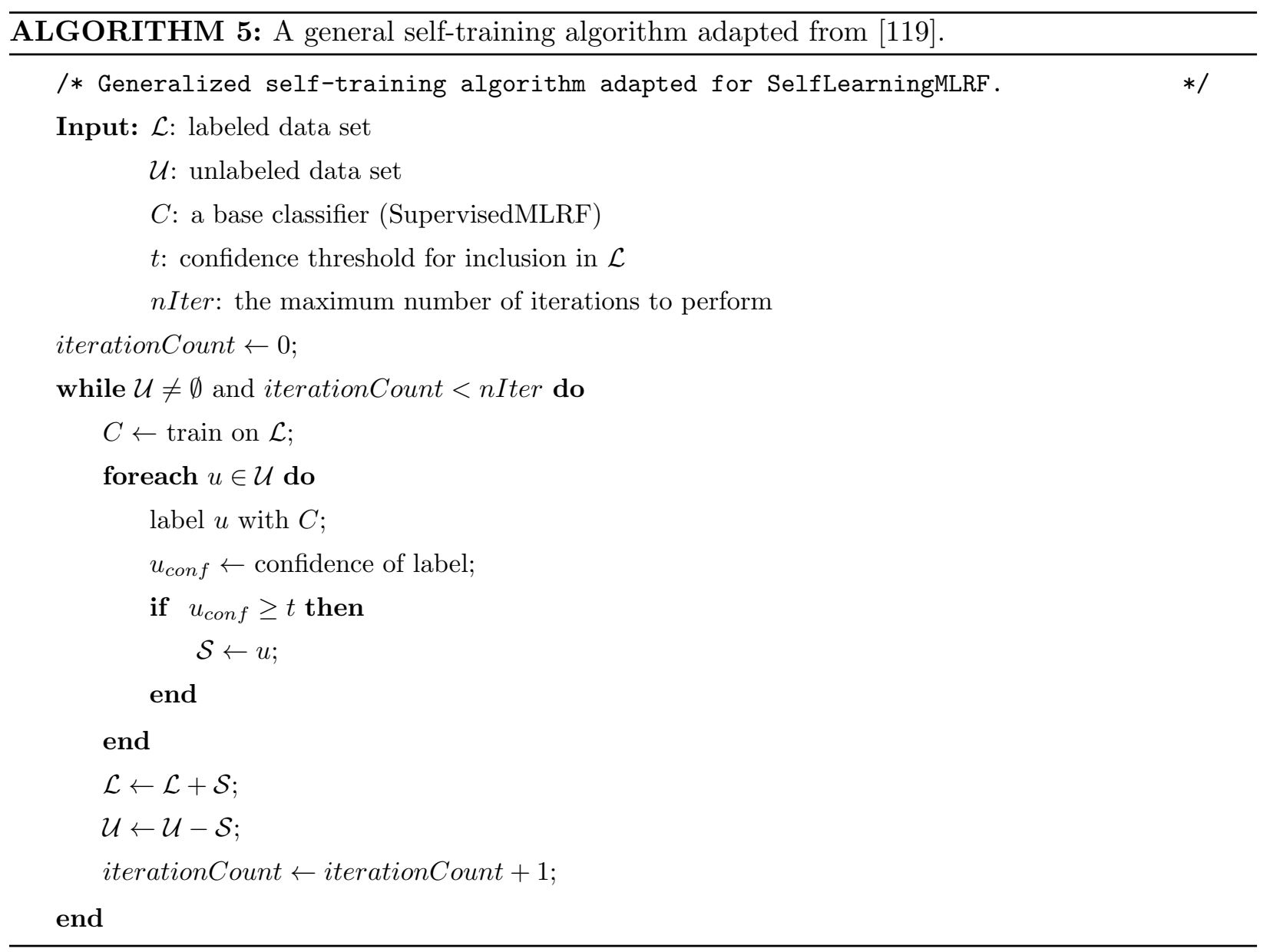

tively. For stopping criteria, we chose three conditions: 1) the unlabeled set is empty, 2) the maximum number of iterations is reached (we chose nIter $=30$ ), and 3) the self-training process encounters "three strikes", where a strike is considered to be an iteration with less than three high-confidence labels found. These specific criteria values were selected after several parameter tuning experiments suggested they would strike a balance between time spent training and classification performance. The result of the algorithm is a RandomForest model that can be used for inductive prediction on unseen data.

\subsection{Results}

To evaluate the effectiveness CoDRIFt and compare its performance to the classifiers presented in Section 9.3, we conducted a series of experiments using binary class versions of either or both of the GBT350Drift and PALFA benchmark data sets (described in Section 7.3). 
In this section, we provide descriptions of the experiments performed and evaluations of each classifier's performance.

\subsubsection{Serial \& Distributed Computing Environments}

The Collective Classification algorithms were evaluated in serial form on a single desktop workstation with $64 \mathrm{~GB}$ of RAM and an Intel@ Core $^{\mathrm{TM}_{\mathrm{i}} 7-7800 \mathrm{~K}} \mathrm{CPU}$ overclocked to 4.5 GHz with eight logical cores. CoDRIFt, SupervisedMLRF, and SelfLearningMLRF were implemented in a distributed computing environment consisting of software installed on the distributed system described in Section 8.3.1. As in Section 8.2.1, we implemented all of our code for Spark (version 2.3.2) in Scala (version 2.11). We used the Scala Integrated Development Environment for Eclipse $\square^{7}$ as our development environment with Maven $\bigsqcup^{8}$ for integrated dependency management. Several packages used in our code were taken or adapted from Apache Spark's scalable machine learning library (MLlib).

\subsubsection{CoDRIFt Results}

We evaluated CoDRIFt on both the GBT350Drift and PALFA data sets to lend a small amount of generalizability to our results. After some preliminary, parameter tuning experiments, we chose several static values for variables in the CoDRIFT algorithm. Specifically, we set the minimum number of trees in the final model to 75 , based on our results (see Section 9.4.3 that using less than 50 trees was detrimental to performance. We also chose recall as the optimization parameter, as very high recalls are extremely important in radio pulsar astronomy. We used SupervisedMLRF as our base RandomForest classifiers and set the number of trees in each SupervisedMLRF to ten, for performance reasons. Since CoDRIFt may potentially construct hundreds of SupervisedMLRF models before settling on a final set of trees, and our results in Section 9.4.5 showed that training times directly increase with the number of trees, allowing more trees per model would significantly increase CoDRIFt's training time. By having so many models generated, we still hoped to maintain the advantages in classification performance of having many trees. Finally, we allowed $k$ to

\footnotetext{
${ }^{7}$ http://scala-ide.org/

${ }^{8}$ https://maven.apache.org/
} 
vary as the number of executors. Since $k$ represents the number of partitions for the RDDs, and Spark recommends two partitions per executor, we set $k$ for each experiment to be twice the number of executors.

For the floating variables in our trials, we varied the labeled percent of the data from $1 \%$ to $15 \%$ and the number of executors from 1 to 25 in increments of five and repeated each each experiment five times, resulting in a total of 220 experiments. For each experiment, we first split the data with stratified random sampling into $70 \%$ model-building and $30 \%$ testing sets. The model-building set was then split again (70\%/30\%) using stratified random sampling to create a training and evaluation set for use according to the CoDRIFt algorithm (see Algorithm 2). Finally, the training set that remained was split (again with stratified random sampling) into labeled and unlabeled sets according to the labeled percentage of the particular experiment.

\section{CoDRIFt Classification Performance Results}

The results of our experiments with regard to classification performance are shown in Figure 9.1, using mean recall (top) and F-4 scores (bottom) for performance metrics. The results show that CoDRIFt is very good at classifying positive examples of single pulses from pulsar sources, especially when the percent of the data given with labels is very low and more than one executor is allowed for processing. CoDRIFt generally performs better with less labeled data! However, since the algorithm is designed to optimize recall, this performance improvement comes at the expense of reduced precision.

The reduced precision scores are evident in the bottom plot of Figure 9.1, which provides mean F-4 scores. As detailed in Section 2.2.4, the F-4 score is a harmonic mean between the recall and precision, with the recall weighted four times more than the precision. In the figure, the low precision scores can be seen "pulling down" the high recall scores. F-4 scores steadily drop as more executors are allowed for some of the PALFA trials. (Note the difference in scale between Figure 9.1 top and bottom.) The F-4 scores also tend to decrease as the percent of labeled data decreases. While, in a perfect world, we would like the precision to be as high as the recall, for classes with extremely imbalanced data, this situation is not undesirable and the plots shown can be deceptive. All of our performance measures 

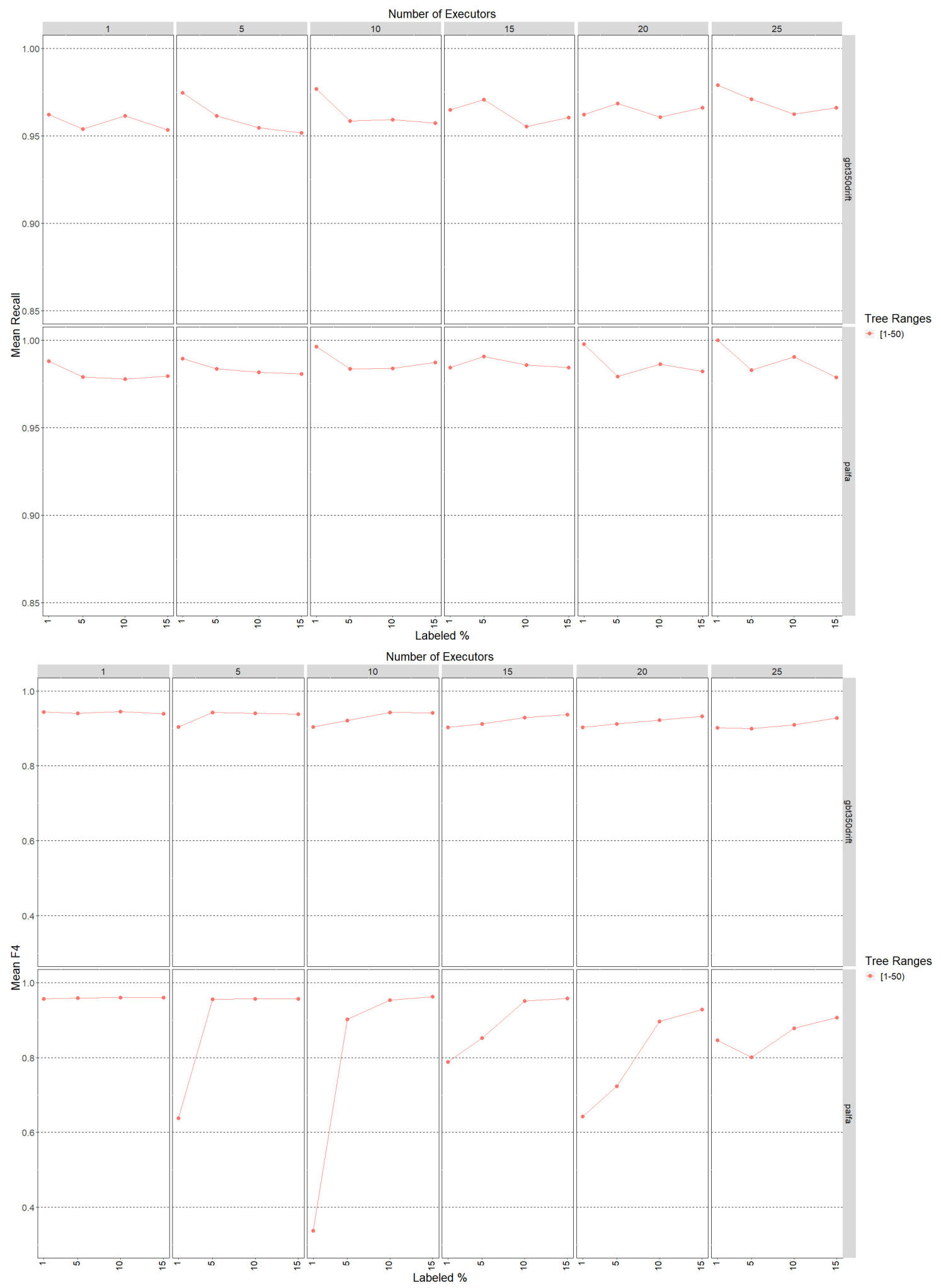

Figure 9.1: Mean recall (top) and F-4 scores (bottom) scores for CoDRIFt models built on training sets from the data for GBT350Drift and PALFA with the percent of labeled instances ranging from $1 \%$ to $15 \%$. 
reported consider only the performance of the model with respect to the minority class. This is because the extremely high scores reported for the majority class would dominate the results. For example, a classifier that labeled every instance as the majority class would have very high recall (0.97) and perfect precision (1.0) if there were 30,000 non-pulsars and only 1,000 pulsars. In reality, one of our worst performing models with respect to precision (0.27) only mis-classified eight of the 274 positive instances with 685 false positives and 29,992 correctly classified negative instances. For problem domains with extreme data imbalances, lower precision is a small price to pay for near-perfect recall of the minority class.

\section{CoDRIFt Execution Performance Results}

With respect to execution performance, we report the mean training times (top) and testing times (bottom) for our CoDRIFt models in Figure 9.2. As can be seen in Figure 9.2, training times increase when less of the data is labeled. This is due to the larger number of unlabeled instances that must be labeled by every tree in every RandomForest created in the multiple iterations of a CoDRIFt training run. What may seem counter-intuitive is the increase in training times as the number of executors increases. This is an artifact of the way we chose to assign the value $k$ from Algorithm 2 in our experiments. We chose $k$ to be double the number of executors for each trial, based on Spark's partitioning recommendation. Since the number of RandomForest models created is $2 * k$, they increase by a factor of four as the number of executors increases. While the training times are very high when using so many models, this is representative of the difficulty of finding a sufficient group of trees for the final CoDRIFt model. Note that high training times are exhibited by several other semi-supervised algorithms, as discussed in Sections 9.4.3 and 9.4.5.

As shown in Figure 9.2, while testing times remain very small, in most cases, models built with more labeled data take slightly longer to test. Upon examination of the decision trees created for several CoDRIFt models from the $1 \%$ and $15 \%$ labeled, we noticed that the trees built with more labeled data appeared to be more complex, i.e., they contained many more trees with a large number of nodes. This added complexity could cause an increase in testing times for the cases examined. 

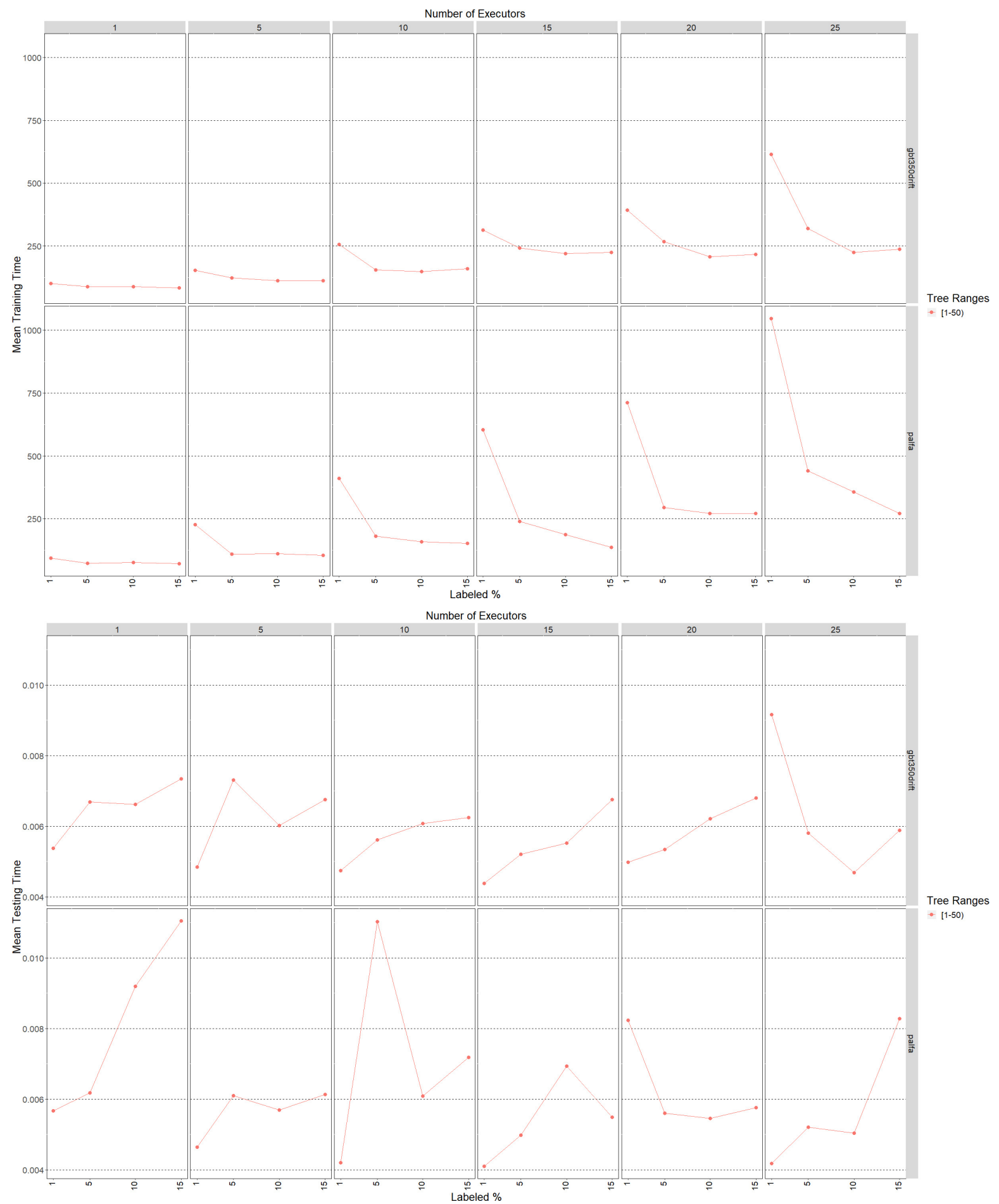

Figure 9.2: Mean training (top) and testing (bottom) times for CoDRIFt models built on training sets from the data for GBT350Drift and PALFA with the percent of labeled instances ranging from $1 \%$ to $15 \%$. 


\subsubsection{Collective Classification Results}

We evaluated the serial, semi-supervised classifiers presented in Section 9.3.1 with a series of trials on different versions of our PALFA benchmark. Each benchmark was randomly sampled (stratified) with different proportions of labeled to unlabeled instances. For each version, we removed the labels from a given percentage of randomly selected instances, resulting in data sets containing: $1 \%, 5 \%, 10 \%, 25 \%, 50 \%, 75 \%, 90 \%, 95 \%$, and $99 \%$ labeled instances. We then evaluated the classification and execution performance of the nine Collective Classification classifiers on each data set with no repetitions for a total of 81 experiments.

\section{Collective Classification Performance Results}

For ease of comparison to the CoDRIFt results, in Figure 9.3 we provide a limited set of Recall scores for nine Collective Classifiers focusing on the experiments with lower percentages of labeled data. The results show that the best classifier with only one percent of the data labeled was CollectiveForest with a recall of 0.80 , which is almost $18 \%$ worse than the average score for CoDRIFt over the PALFA and GBT350Drift data sets. In general, the results of the Collective Classifiers built with very small amounts of data are mediocre at best and abyssmal at worst, with the AdvancedCollective achieving a recall of 0.0 with one percent labeled data. Overall, CoDRIFt models improved recall over Collective Classifiers by an average of $30 \%$ in a direct comparison of similar experiments.

Figure 9.4 shows line plots of the raw Recall and F-Measure (F-1) scores for the full range of our experiments with Collective Classifiers. The results show that, in general, the semisupervised learning classifiers can achieve high classification performance without requiring $100 \%$ of the data to be labeled. Scores for several classifiers suffered little classification performance degradation with up to $75 \%$ labeled instances. However, classification performance decreased as the labeled percentage dropped below $75 \%$. Even with the classification performance degradation, F-Measure scores for the CollectiveForest and CollectiveWoods classifiers with only one percent labeled data remained over 0.85. Overall, CollectiveForest consistently scored the highest marks of any semi-supervised learning classifier for the given 


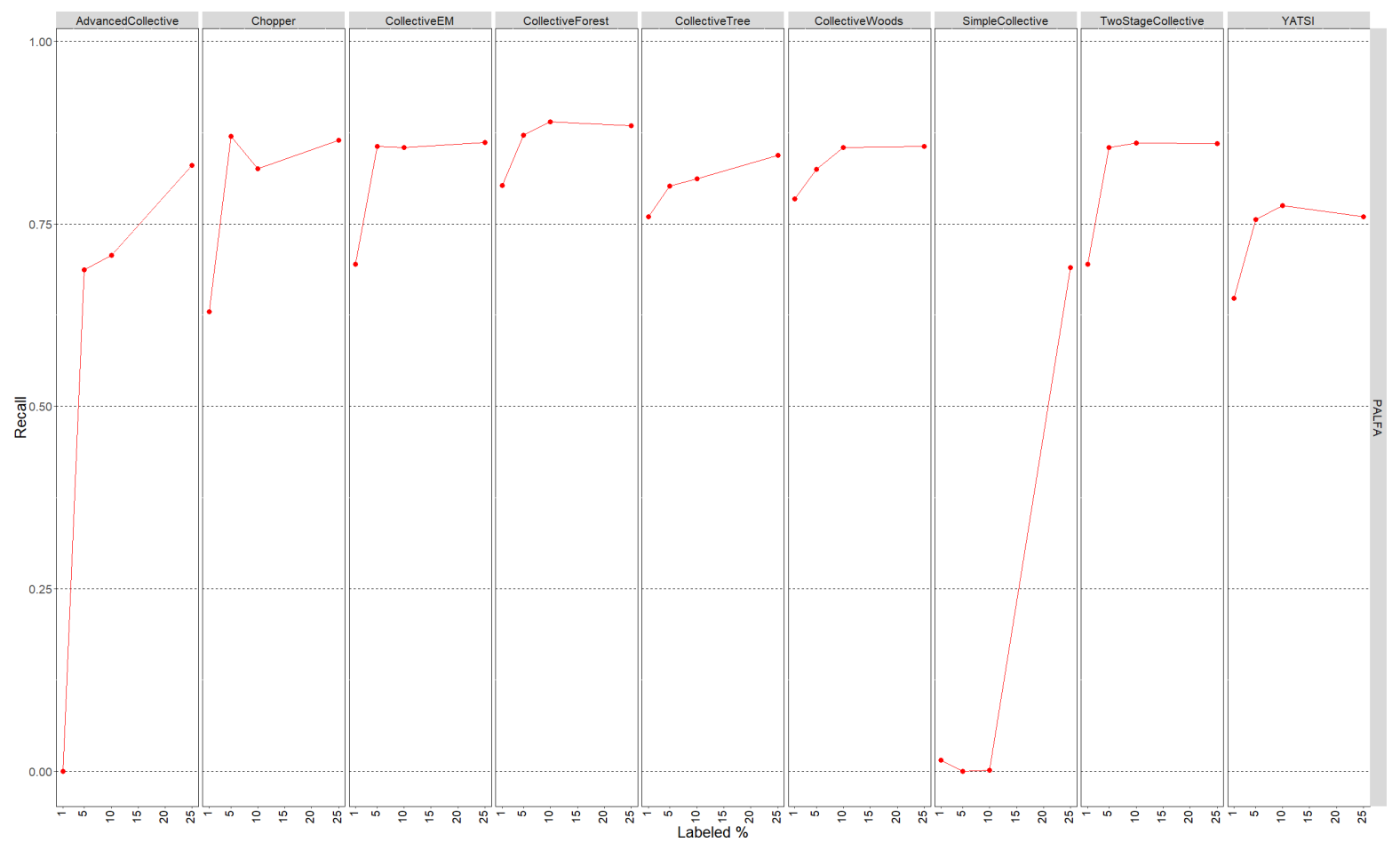

Figure 9.3: Recall scores for nine Collective Classifiers (one per panel) built on training sets from the PALFA data with the percent of labeled instances ranging from $1 \%$ to $25 \%$.

data.

\section{Collective Execution Performance Results}

The training (top) and testing (bottom) times of the Collective Classifiers with low percentages of labeled instances are shown in Figures 9.5. With respect to training times, the best and worst performers overall are CollectiveTree and AdvancedCollective, respectively. However, results are inconsistent for other classifiers, with some training faster with less labeled instances, some training slower with less labeled instances, and others showing little effect. The training times for CollectiveForest, which had the best classification performance, remain consistently in the "middle of the pack" regardless of labeled percentage. Note that five of these classifiers exhibit very long training times. While these results are not directly comparable to CoDRIFt and SelfLearningMLRF experiments with multiple executors because Collective Classification is serial code running on a single workstation rather than parallel code running on a distributed system, they still seem to indicate that longer training 

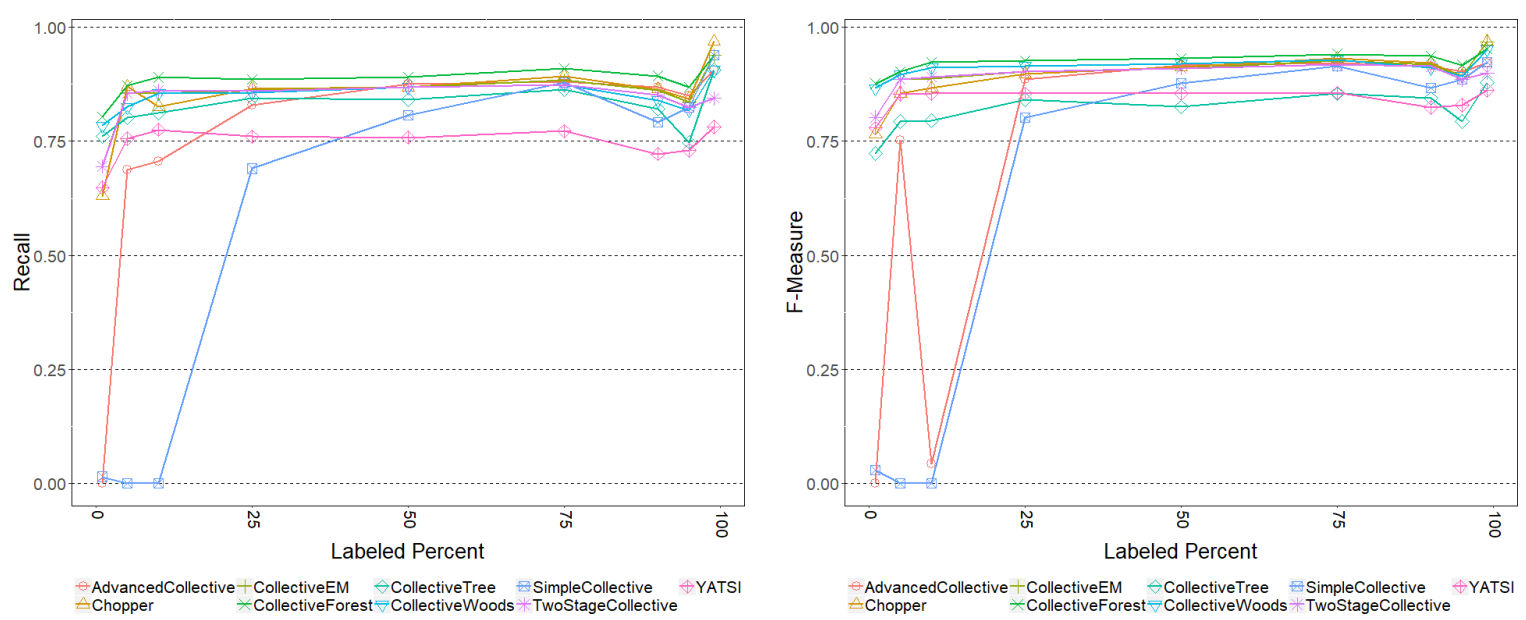

Figure 9.4: Recall (left) and F-Measure (right) scores for nine different semi-supervised learning classifiers on the PALFA binary benchmark with varying percentages of labeled instances, given by the $x$-axis.

times are common for semi-supervised learning models.

While the scale makes it difficult to see, testing times for most Collective Classifiers were on par with times reported for CoDRIFt, with the exception of three classifiers. In general, for the Collective Classifiers execution performance is the best with a higher percentage of labeled instances and falls off as more unlabeled data is present. YATSI exhibits the worst performance overall, with testing times much higher than the other classifiers. The testing times for CollectiveForest steadily increase as the unlabeled percentage increases, eventually becoming the second worst performer for $10 \%$ or fewer labeled instances. However, CollectiveForest testing times still remain significantly lower than YATSI, which exhibited the largest increase in testing times.

\section{Difficulties with Collective Classification}

The biggest drawback of the versions of Collective Classification algorithms we tested (detailed in Section 9.3.1) was that they can only perform transductive semi-supervised learning. As mentioned in Section 2.2.3, transductive semi-supervised learning predicts only the labels of instances present in the training set and cannot be used to generate a predictive model for future unseen data. In order to make predictions for all of the unlabeled data in the PALFA set, all six million instances must be included in the training set. However, due 

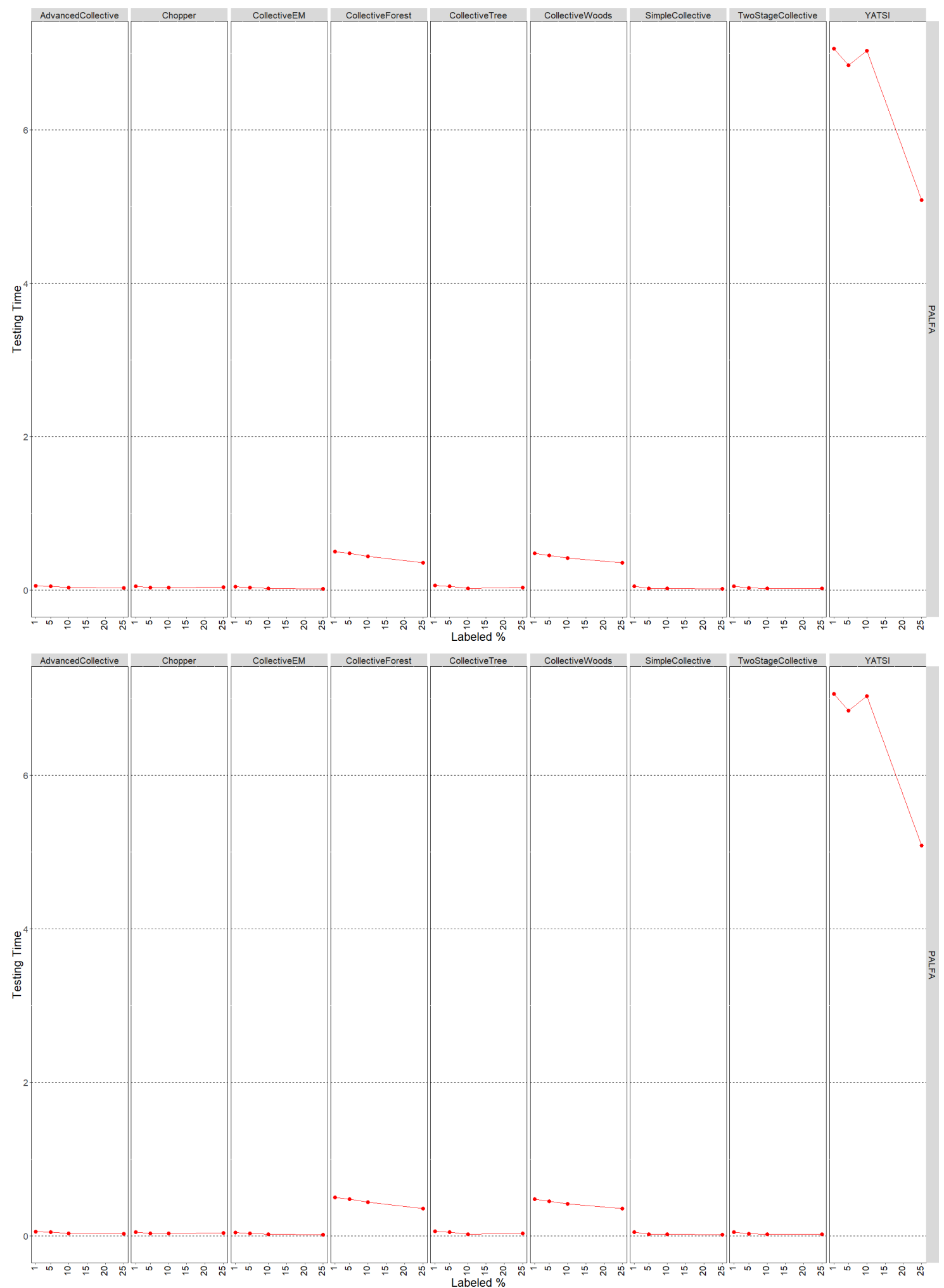

Figure 9.5: Training (top) and testing (bottom) times for nine Collective Classifiers (one per panel) built on training sets from the PALFA data with the percent of labeled instances ranging from $1 \%$ to $25 \%$. 
to implementation limitations, such large training sets inevitably crashed the Java Virtual Machine (JVM) running the experiment. The critical errors occurred when the JVM running any of the given Collective Classifiers attempted to process data sets with one quarter of the size of ours. With such large data sets, the JVM spent over $98 \%$ of its time doing garbage collection with less than $2 \%$ of the used memory being freed, even when available memory was more than sufficient to handle the data set (64 GB total RAM). Additionally, the Collective Classifiers we examined were not suitable for scalable pulsar classification due to their single threaded, serial implementations.

\subsubsection{SupervisedMLRF Results}

Since SupervisedMLRF is used as the base classifier in our CoDRIFt implementation, we spent additional time and resources to fully test the classifier in a variety of conditions. Our experiments used both the GBT350Drift and PALFA benchmark data sets. These experiments were exploratory in nature, as we sought insight into how distributed RandomForest models would behave under the following variables: percent of data used for training, number of trees used to construct the forest, and number of distributed executors allowed for processing. In each trial, we performed stratified random sampling to select subsets of the data for training and testing. To fully see how the algorithm performed with less and less training data, we split the data to allow training on $1 \%, 5 \%, 10 \%, 15 \%, 25 \%, 35 \%, 45 \%$, $55 \%, 65 \%, 75 \%, 85 \%$, and $95 \%$ and reserved the remainder of each split for testing. For each subset of training data, we performed separate trials varying the number of trees in each forest from 10 to 200 by increments of ten, and each of those trials was performed with a given number of executors, ranging from 1 to 25, increasing by five each time. Each trial was repeated ten times for a total of 28,800 trials.

\section{SupervisedMLRF Classification Performance Results}

For our overall analysis of SupervisedMLRF, Figure 9.6 shows boxplots of the results of the experimental trials using training samples from 25\% to 95\%. For the GBT350Drift data, F-4 values are consistently high, but show an interesting trend of performing better with less 
training data than with more. This trend is more pronounced in Figure 9.7, which reports only the mean values for each group. In the PALFA data, scores remain very high across the board. For both data sets, performance drops for models trained on only one percent of the data, as evidenced in Figure 9.7. In reference to the number of trees used to construct the models, forests using less than fifty trees consistently perform worse than those with more trees. Interestingly, models trained with the largest amount of training data exhibit the greatest variance, a result that appears contradictory to a commonly accepted property of RandomForests, i.e., that their variance should be low due to the aggregation or bagging of the votes across the ensemble of learners. We suspect that the trees trained with large amounts of labeled data grew more complex, as witnessed on a smaller scale in trees for several CoDRIFt models reported in Section 9.4.3.

Figure 9.8 provides results for the recall (top) and F-4 (bottom) scores of SupervisedMLRF and are directly comparable to CoDRIFt's results. The mean recall scores for SupervisedMLRF are consistently lower than those of CoDRIFt, especially for the trials with labels assigned to only one percent of the data. On average, CoDRIFt models showed a $4.0 \%$ improvement to recall with one percent of the data labeled and a $2.0 \%$ improvement over the SupervisedMLRF classifiers with five percent of the data labeled. The F-4 scores in Figure 9.8, however, are consistently higher than those achieved by CoDRIFt. On average over all trials, SupervisedMLRF F-4 scores are almost $4.9 \%$ higher than those of CoDRIFt. This is due to the low precision problems with CoDRIFt models discussed in Section 9.4.2.

\section{SupervisedMLRF Execution Performance Results}

A fair comparison of training times (see Figure 9.9) between SupervisedMLRF and CoDRIFt cannot be made. SupervisedMLRF makes only one model in each experiment, whereas CoDRIFt may be generating hundreds of SupervisedMLRF classifiers for each experiment.

As far as testing times are concerned, the results reported in Figure 9.9 (bottom) for SupervisedMLRF models with 50 to 100 trees (green lines with triangles) are significantly larger than the testing times observed for our CoDRIFt models, which generally contained between 75 and 100 decision trees. (Note that the $x$-axis scales for Figure 9.9 range from $1 \%$ 


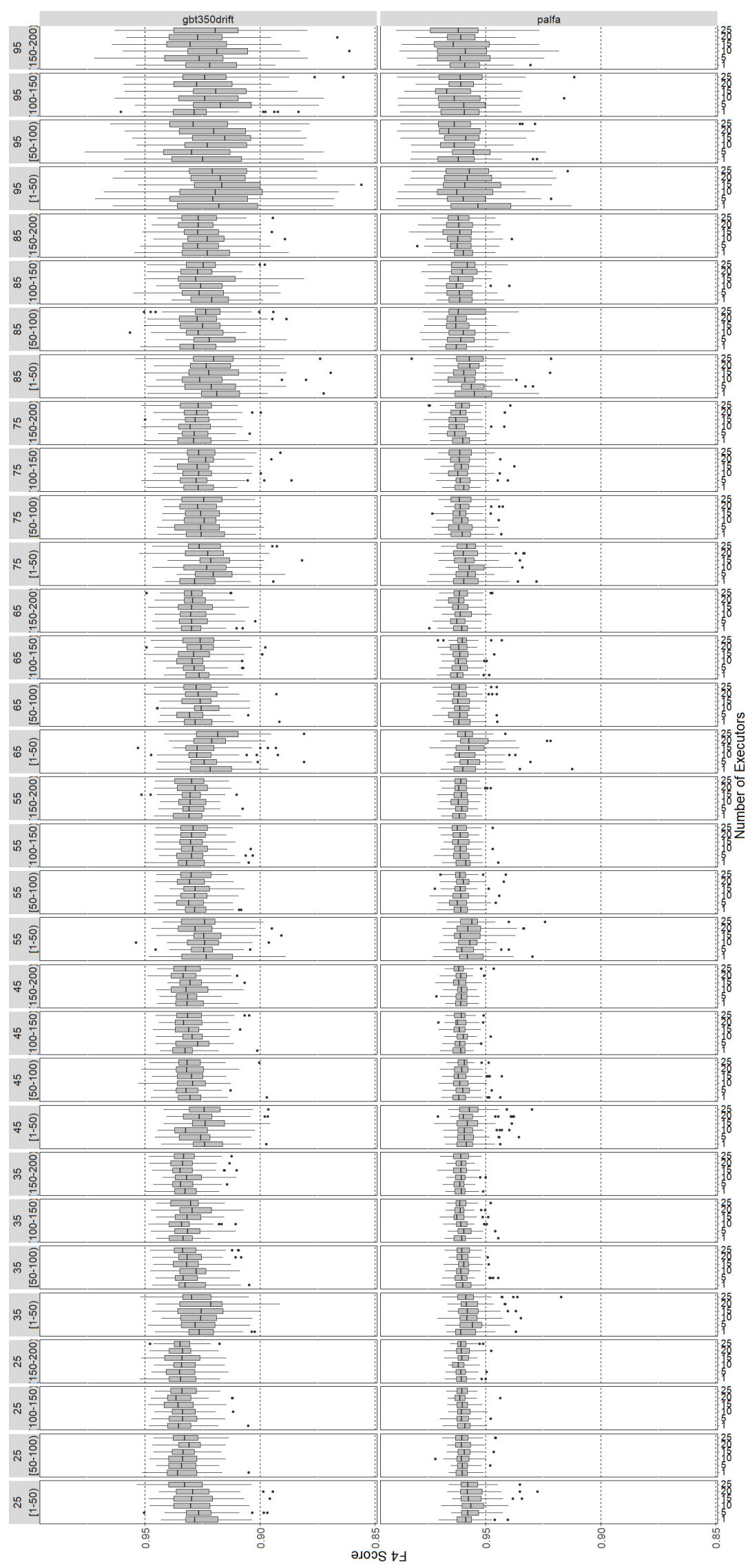

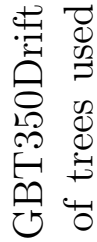

क्ष 8

శై

त्ठ

ส

फ

se $\approx$

Lิ

$\circ+$

万o

ำ

हैं 50

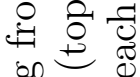

.

สี

更..$\exists$

记

ᄋ o

.

武造

चี के

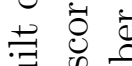

의

a $\frac{9}{\ddagger} \exists$

皆

व

पा है

당

$\sum \stackrel{0}{0}$

. 동

可的

जू है

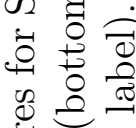

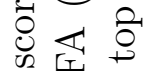

年㟧

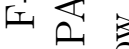

๑े छ

( )

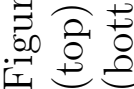




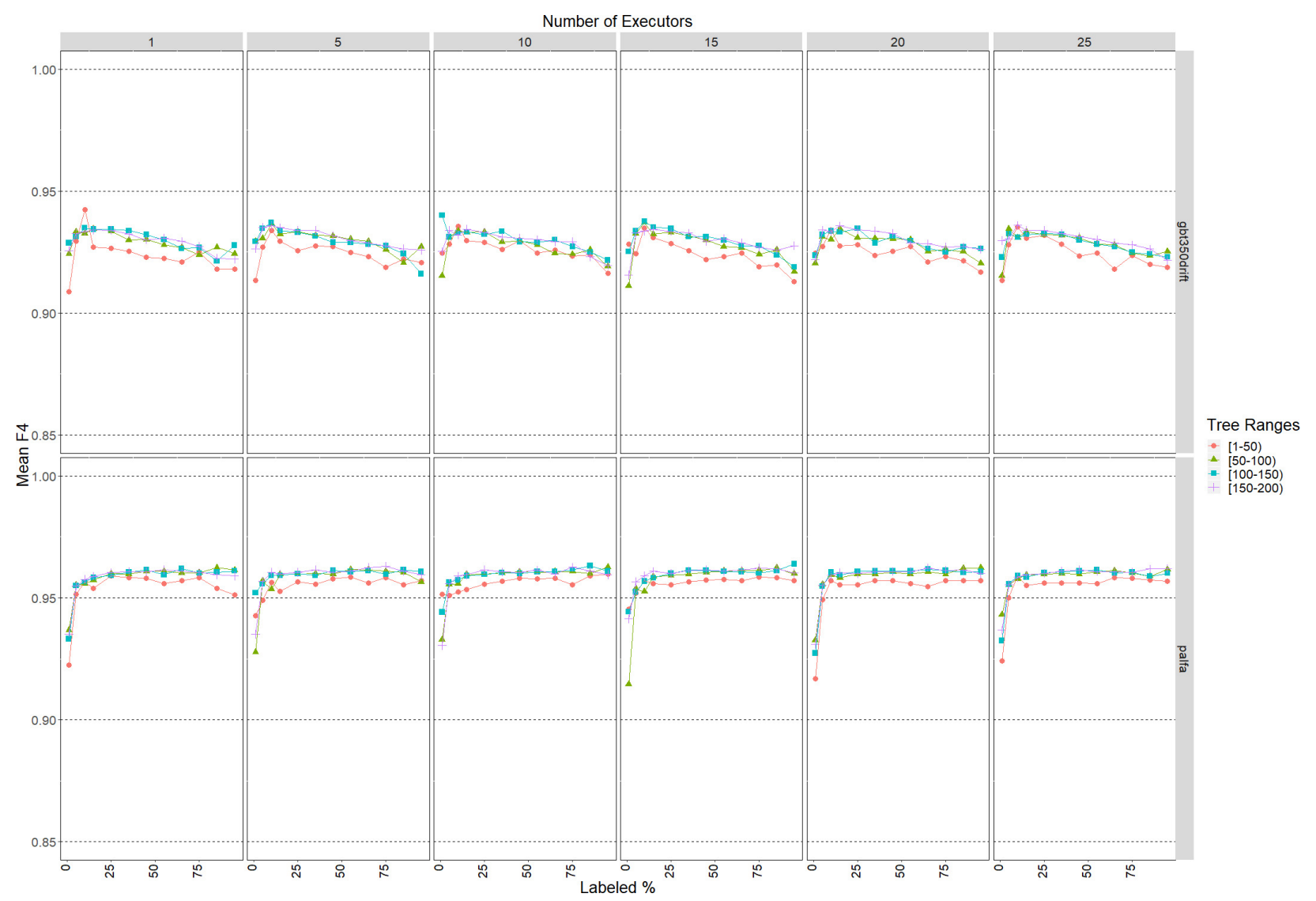

Figure 9.7: Mean F-4 scores for SupervisedMLRF models built on training sets from the data for GBT350Drift (top) and PALFA (bottom) with the percent of labeled instances ranging from $1 \%$ to $95 \%$.

labeled to $95 \%$ labeled.) We attribute this difference to our implementation of CoDRIFt as a much simpler data structure than the Spark MLlib RandomForestModel used for SupervisedMLRF. Our implementation is a simple ListBuffer, a mutable data structure in Scala that is optimized to facilitate the addition and removal of objects. Each tree in the list is a tuple containing a Decision TreeModel and a few other primitives to help us interpret results (i.e., a String indicating whether it is a supervised or semi-supervised tree, a double with the Recall score the tree attained during evaluation, and a double indicating if it was a positive or negative tree). By not implementing CoDRIFt as the more complicated RandomForestModel and by writing our own simple voting function, we avoided the overhead associated with the RandomForestModel that increases testing times.

Several overall insights were gained from our analysis of SupervisedMLRF's execution 

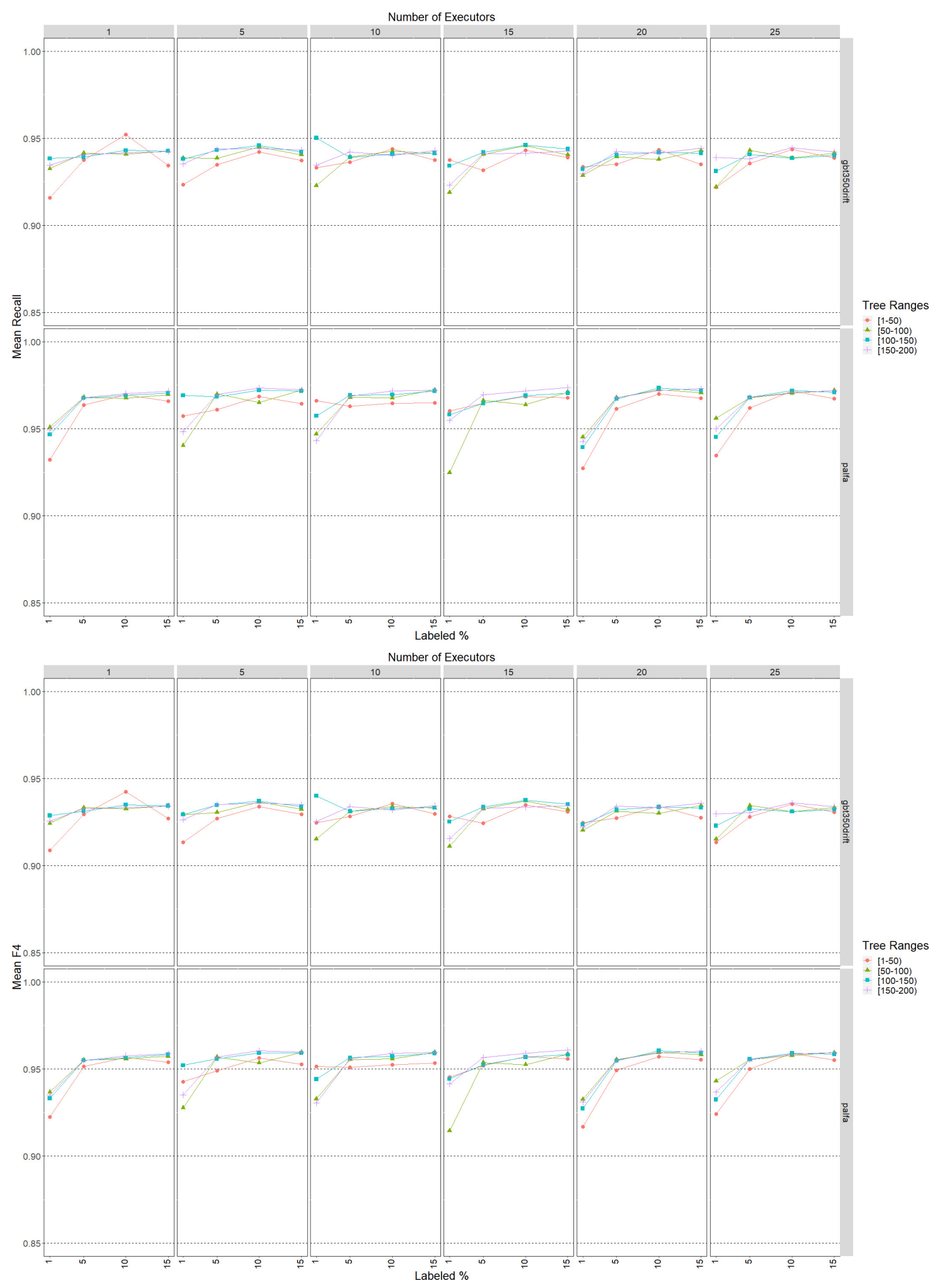

Figure 9.8: Mean recall (top) and F-4 (bottom) scores for SupervisedMLRF models built on training sets from the data for GBT350Drift and PALFA with the percent of labeled instances ranging from $1 \%$ to $15 \%$. 

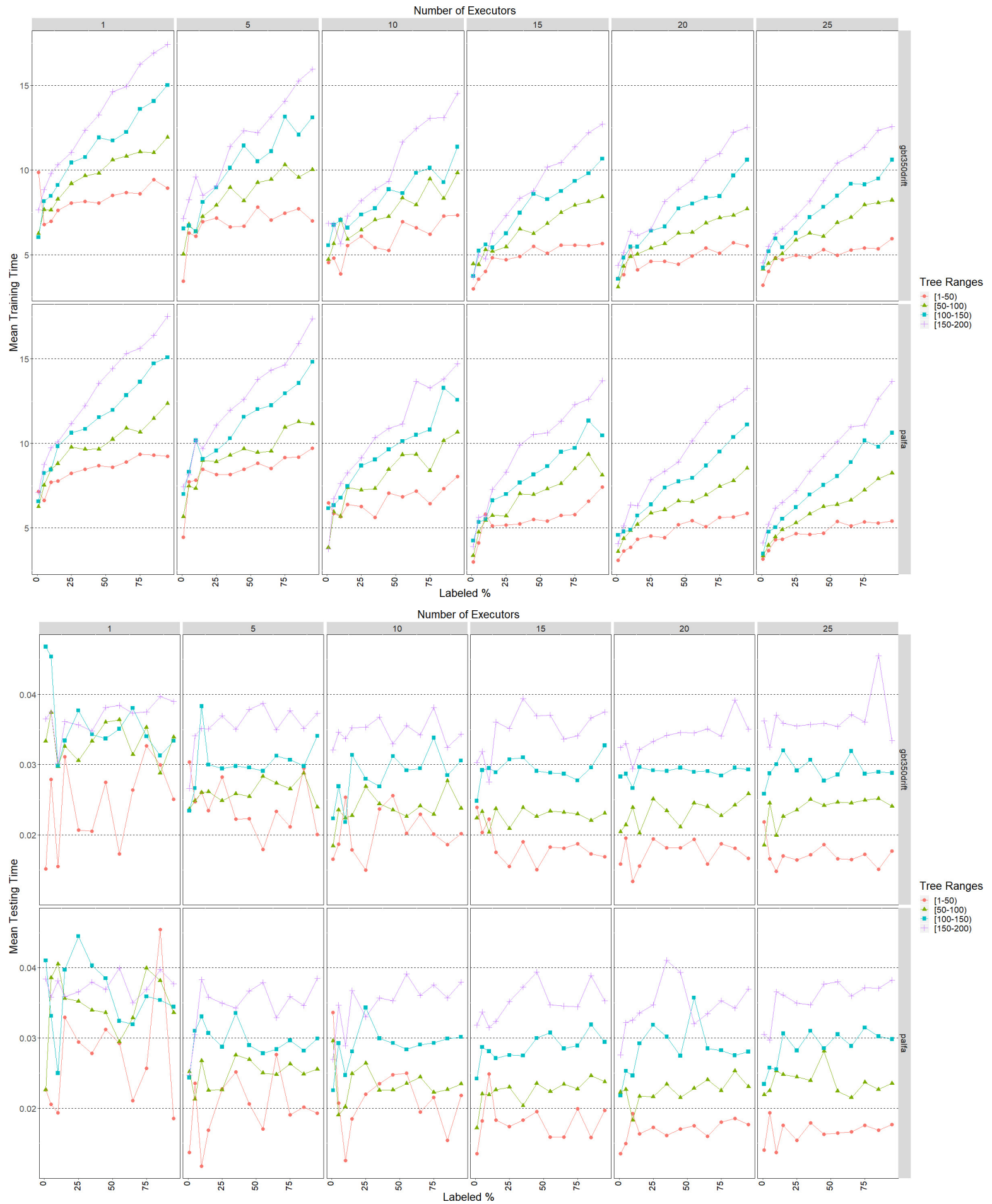

Figure 9.9: Mean training times for SupervisedMLRF models built on training sets from the data for GBT350Drift and PALFA with the percent of labeled instances ranging from $1 \%$ to $95 \%$. 
performance. Training times can be seen to increase linearly with the percent of data used to train the models and also with the number of trees used to construct the models. This makes sense, as a RandomForest model should take longer to train more trees and calculations for split points take longer when there are more instances to consider. The number of executors helped mitigate this increase in training times to an extent, with significant reductions moving from one to fifteen executors. However, adding additional executors over fifteen showed little to no improvement.

Testing times were very fast for all models, classifying all testing instances in less than half a second. Predictably, models with less trees were able to make predictions faster. Since RandomForests require each decision tree to make its own prediction for each instance, more trees means more predictions to calculate and more votes to tally. The addition of more executors did not appear to have a noticeable effect. Examination of the SupervisedMLRF source code showed that these votes are performed in a serial manner and thus gain no benefit from additional distributed resources.

\subsubsection{SelfLearningMLRF Results}

SelfLearningMLRF is our implementation of the most similar algorithm to CoDRIFt in the literature [119]. We used it to serve as a comparison to CoDRIFt. To enable the comparison, we ran a series of experiments on both the GBT350Drift and PALFA data. We focused on training with the same percentages of labeled to unlabeled data (1\% to $15 \%$ ) as CoDRIFt. Since there is only one base classifier (we used SupervisedMLRF as our base) constructed during each iteration of the self-learning algorithm (see Algorithm 5), we opted to vary the number of trees created by each model. We also varied the number of distributed executors allowed for processing, as we did for CoDRIFt in Section 9.4.2. For each percentage of labeled data, we performed experiments allowing the number of trees to vary from 25 to 200 , increasing by 25 , and the number of executors to vary from 1 to 25 , increasing by increments of five. By repeating each experiment five times to validate the results, we performed a total of 1,920 trials for SelfLearningMLRF. For each experiment, $70 \%$ of the data was used for training and $30 \%$ was reserved for testing the models created. 


\section{SelfLearningMLRF Classification Performance Results}

We present the results of our SelfLearningMLRF experiments with respect to classification performance in Figure 9.10, which givea the mean recall (top) and mean F-4 (bottom) scores attained, respectively. On average over all trials, CoDRIFt improved recall scores by $1.5 \%$ over the already very high recall scores achieved by SelfLearningMLRF. CoDRIFt showed an average improvement in recall of $2.9 \%$ through all experiments with only one percent of the data labeled. Much like every other algorithm tested, SelfLearning MLRF's performance tends to drop when the number of available labels for training is very small. CoDRIFt is the only algorithm tested that classifies minority class instances better with very few labeled examples.

SelfLearningMLRF models, on average, received higher marks for precision than CoDRIFt, and similar marks to SupervisedMLRF, as evidenced by the mean F-4 scores (bottom) in Figure 9.10. On average, SelfLearningMLRF had F-4 scores $4.5 \%$ higher than CoDRIFt and $<0.5 \%$ lower than SupervisedMLRF. As mentioned above, for problem domains willing to sacrifice a small amount of precision to avoid false negatives, CoDRIFt is the best algorithm out of those evaluated in this chapter.

\section{SelfLearningMLRF Execution Performance Results}

Mean training times for SelfLearningMLRF classifiers, shown in the top plot of Figure 9.11, range from 100 to 300 seconds for models with more than 50 trees. While these training times are faster than CoDRIFt, the advantage is not significant. CoDRIFt is expected to take longer to train due to the large number of RandomForest classifiers generated, evaluated, and used to label unlabeled data in the algorithm. When training a classifier for 200 or more seconds, an additional minute or two seems to be a good trade-off for improved recall of rare instances in highly imbalanced data set. Additionally, the models created are inductive and therefore could be trained offline after the arrival of new data. Regardless, optimizing CoDRIFt for execution performance is a concern and will be the subject of future work.

In Figure 9.11, we provide the mean testing times (bottom) recorded for SelfLearn- 

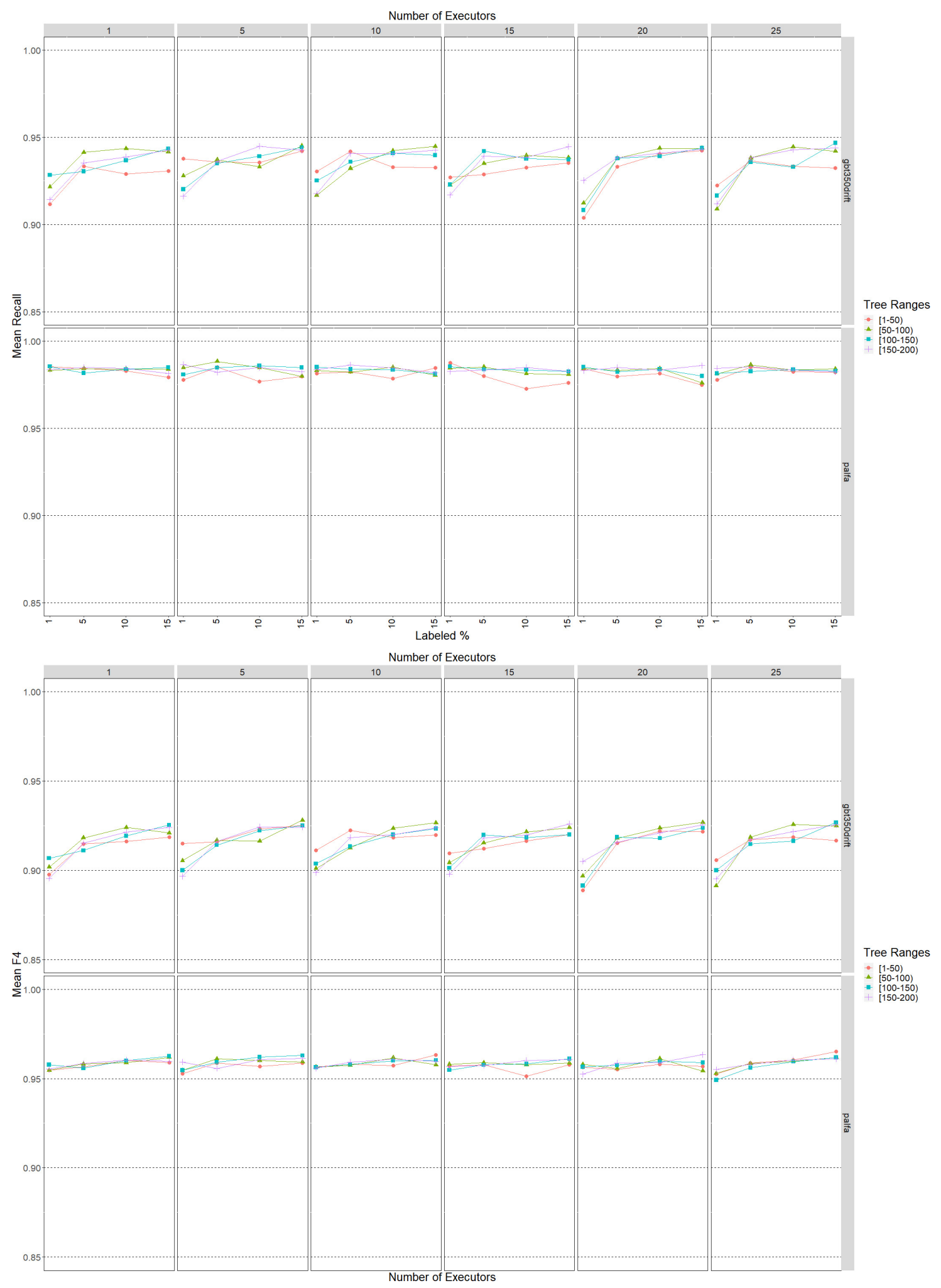

Figure 9.10: Mean recall (top) and F-4 (bottom) scores for SelfLearningMLRF models built on training sets from the data for GBT350Drift and PALFA with the percent of labeled instances ranging from $1 \%$ to $15 \%$. 


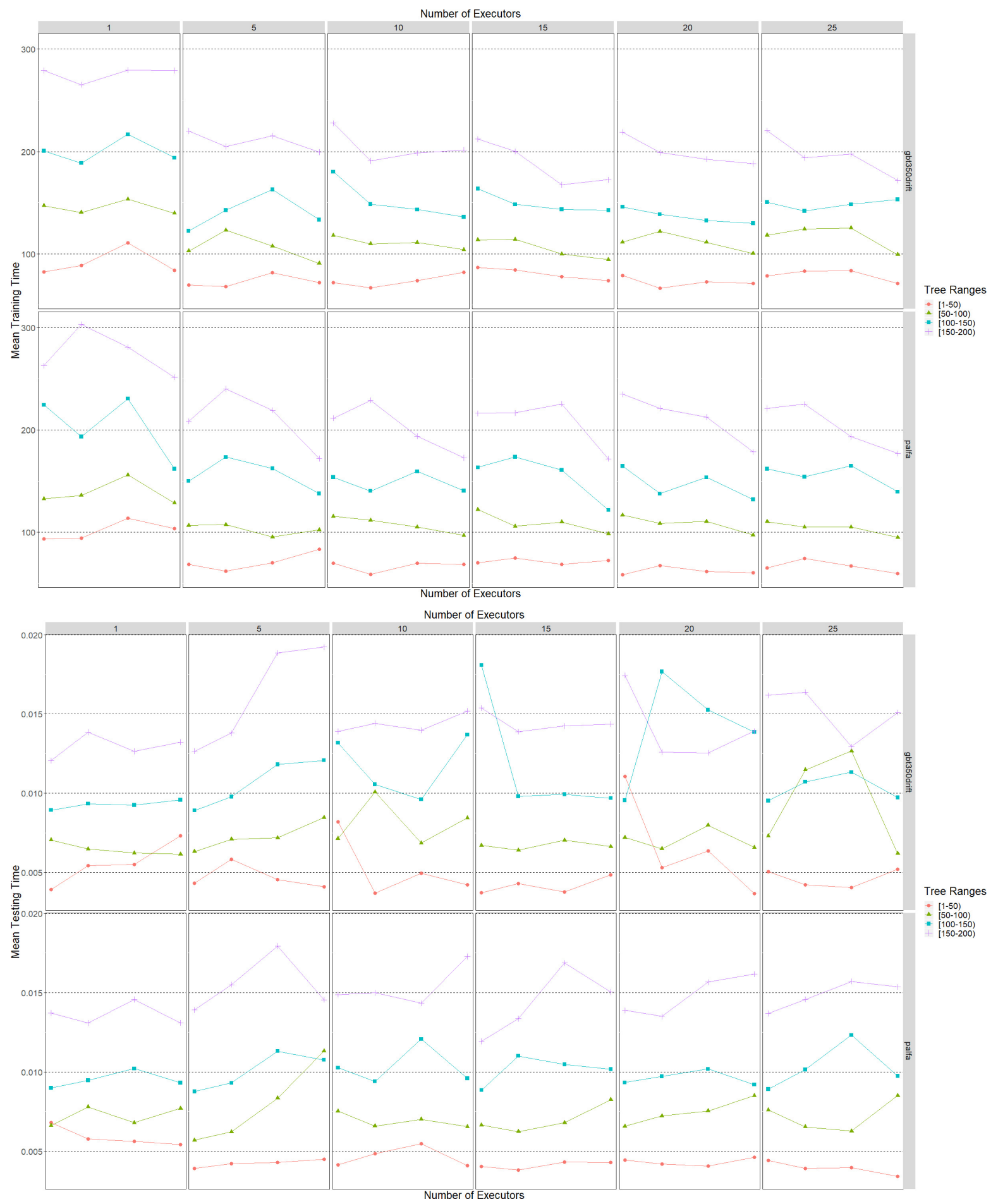

Figure 9.11: Mean training (top) and testing (bottom) times for SelfLearningMLRF models built on training sets from the data for GBT350Drift and PALFA with the percent of labeled instances ranging from $1 \%$ to $15 \%$. 
ingMLRF classifiers in our experiments. As in Section 9.4.4, the results show that testing times for CoDRIFt are slightly lower than those of SelfLearningMLRF. This is likely due to the implementation differences mentioned above between the simpler ListBuffer and the more complicated RandomForestModel data structures used in SelfLearningMLRF.

\subsection{Conclusions}

As the amount of data received by sensitive radio telescopes increases, so do the costs associated with labeling and processing that information. Labeling efforts require staring at plots for countless hours, a well-trained eye, and additional telescope time to find the needles in the cosmic haystack. While human-based labeling of training instances will always be required to some extent, the sheer amount of data received can never be fully examined by human eyes. Semi-supervised learning offers a way for classifiers to leverage machine-labeled data in addition to human-labeled data to accomplish more than either can alone.

In this chapter, we presented CoDRIFt, a novel, distributed, semi-supervised algorithm customized for achieving high recall scores for the minority class in extremely imbalanced data sets. We provided empirical evaluations of CoDRIFt's performance by applying it to the radio pulsar candidate classification problem using benchmark SPE data from the GBT350Drift and PALFA surveys. We designed our experiments to progressively limit CoDRIFt's access to human-labeled data. CoDRIFt responded very well to the experiments, with recall values increasing as the percentages of labeled data decreased. With extremely limited amounts of labeled data and a large amount of unlabeled data, CoDRIFt achieved very high recall scores (0.98 average recall using only one percent labeled data).

We compared CoDRIFt's performance with nine serial, semi-supervised algorithms, one distributed supervised algorithm, and SelfLearningMLRF, our distributed implementation of an existing semi-supervised machine learning algorithm. Our experimental evaluation of CoDRIFt showed that it outperformed all of the other algorithms tested with respect to recall, maintaining high classification performance with only one percent of the data labeled. CoDRIFt achieved recall scores better than the next best classifier (SelfLearningMLRF) by $2.9 \%$ and exhibited an average improvement of almost $35 \%$ over all eleven classifiers. The 
execution performance results showed that CoDRIFt scaled well, and while taking longer to train than simpler supervised algorithms, training times were on par with SelfLearningMLRF. 


\section{Chapter 10}

\section{Conclusion \& Future Work}

"It gave me great pleasure to tell you about the mysteries with which physics confronts us. As a human being, one has been endowed with just enough intelligence to be able to see clearly how utterly inadequate that intelligence is when confronted with what exists. If such humility could be conveyed to everybody, the world of human activities would be more appealing." - Albert Einstein to Queen Elizabeth of Belgium, September 19, 1932

\subsection{Motivation}

The journey of this dissertation began a decade ago, in 2010, when the author spent a summer living in a bungalow on a mountain in the jungles of Arecibo, Puerto Rico. While working as an undergraduate computer science researcher at the (then) largest radio telescope in the world, he discovered that there was a massive potential synergy between the fields of computer science and radio astronomy. Computer scientists are the tinkerers, who love to spend hours analyzing and tweaking algorithms and running experiments to create artificial systems that can help us understand our world. Astrophysicists are the dreamers, whose mathematical and abstract minds are driven to seek out new discoveries that help us understand our world. We both have the same goal, and it is only natural that we work together to achieve it.

Finding pulsars in radio astronomy is exceedingly difficult, and many obstacles exist along the way for those who attempt to uncover Nature's secrets. Some of these obstacles 
are man-made, arising from the sheer volume of data being collected by ingeniously sensitive instruments all over the planet. Big Data technology goes hand in hand with big challenges, bringing with it identification and classification tasks that are visually impossible for a single human to process and computationally infeasible for a single machine to solve. In addition to the obstacles introduced by technology, Nature provides many more obstacles of her own. Space is unfathomably large and defies containment by any amount of Big Data. In the vast expanse of space, radio pulsar signals are extremely rare and often very faint, a result of traveling millions of light years through time and space to reach us.

These obstacles can only be surmounted if the dreamers and the tinkerers combine their efforts. This dissertation represents a decade of the efforts of one such tinkerer, trying to do his part to help us understand our world.

\subsection{Main Contributions}

The goal of the work in this dissertation was to develop machine learning approaches to efficiently search for transient signals in very large, imbalanced radio astronomy data sets. We achieved this goal by:

- developing RAPID, a novel algorithm for candidate identification in radio astronomy data,

- proposing the first machine learning approach for single pulse classification,

- evaluating the performance of existing supervised machine learning techniques for candidate classification,

- developing D-RAPID, a novel, distributed algorithm that parallelizes the process of candidate identification,

- evaluating existing serial and parallel semi-supervised machine learning techniques for pulsar classification, and

- developing CoDRIFt, a novel, distributed, semi-supervised machine learning method for classification in imbalanced datasets. 


\subsection{Future Work}

Our future plans focus on three directions: 1) eliminating performance bottlenecks in our single pulse search approach, 2) improving and further evaluating CoDRIFt, and 3) expanding our work to online learning with data streams. In reference to our scientific workflow (see Figure 6.2 from Section 6.1), we have successfully parallelized the third and fourth stages (peak identification with D-RAPID and candidate classification with CoDRIFt) in this dissertation. In future work, we first plan to parallelize the second stage, Clustering. This work is already under way, and close to being completed. Elimination of this bottleneck will greatly increase the utility of the techniques developed throughout this dissertation.

Second, we plan to further improve the execution and classification performance of CoDRIFt. We believe we can further optimize execution performance by refactoring the code to minimize training times and performing additional parameter tuning experiments. We also want to explore how to improve classification performance with respect to precision, if possible. To further establish the generalizability and effectiveness of the algorithm, we also plan to evaluate it further in both astronomy and non-astronomy applications. To accomplish this, we will test CoDRIFt on additional radio pulsar data sets, attempt to identify and classify additional astronomical phenomena, such as Fast Radio Bursts, and try to evaluate CoDRIFt on additional very large, imbalanced data sets from other problem domains, including credit card fraud detection and cybercrime detection.

Finally, we plan to explore options for real-time searching by altering our approach to work with data streams. One of the reasons we chose Spark as a platform, in addition to the advantages of in-memory data transformation, was support for streaming data analysis with the Spark Streaming extension ${ }^{1}$. While our current approach depends on several preprocessing steps before identification and classification can begin, we plan to explore if and how our techniques could be adapted to work on raw data streams for real-time radio data processing.

\footnotetext{
${ }^{1}$ https://spark.apache.org/streaming/
} 
Appendix: Additional Figures 

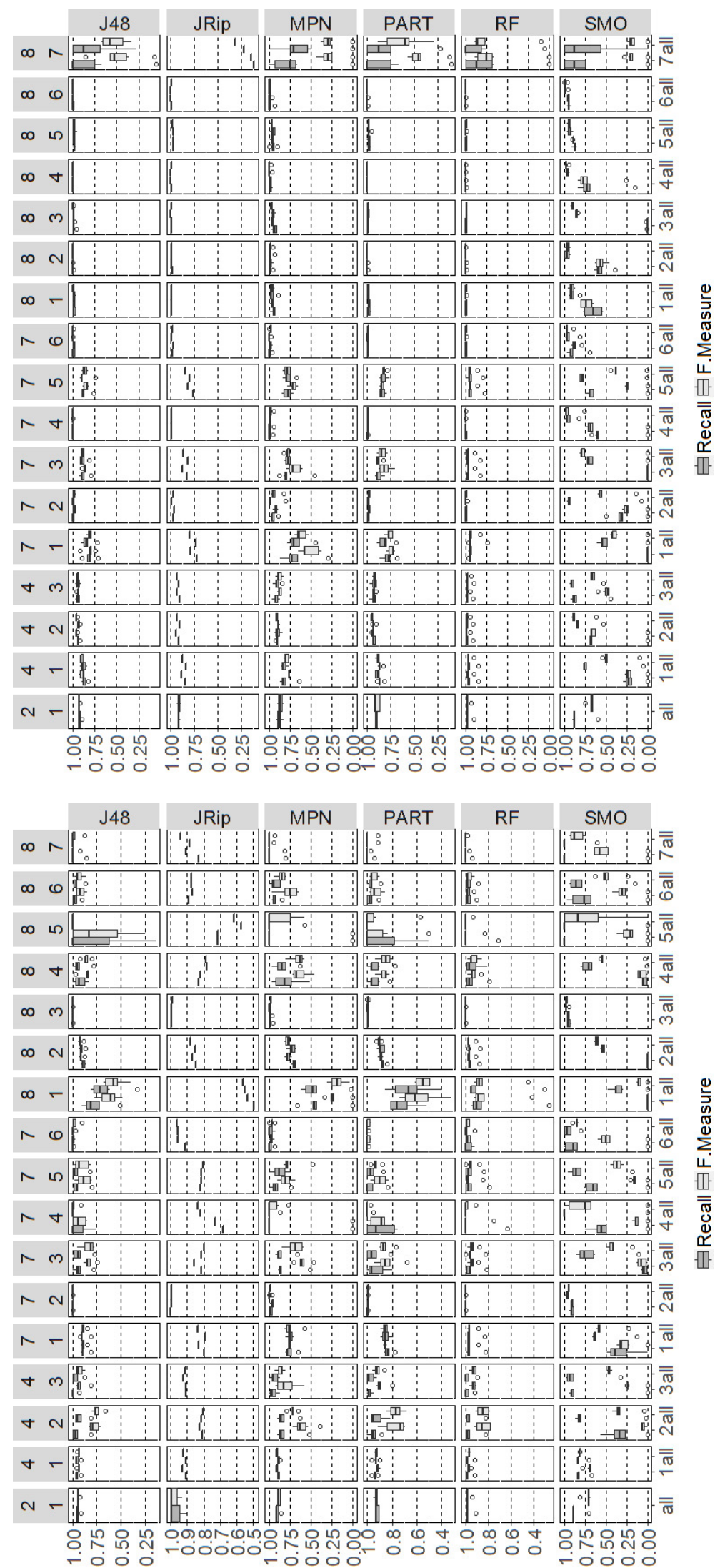

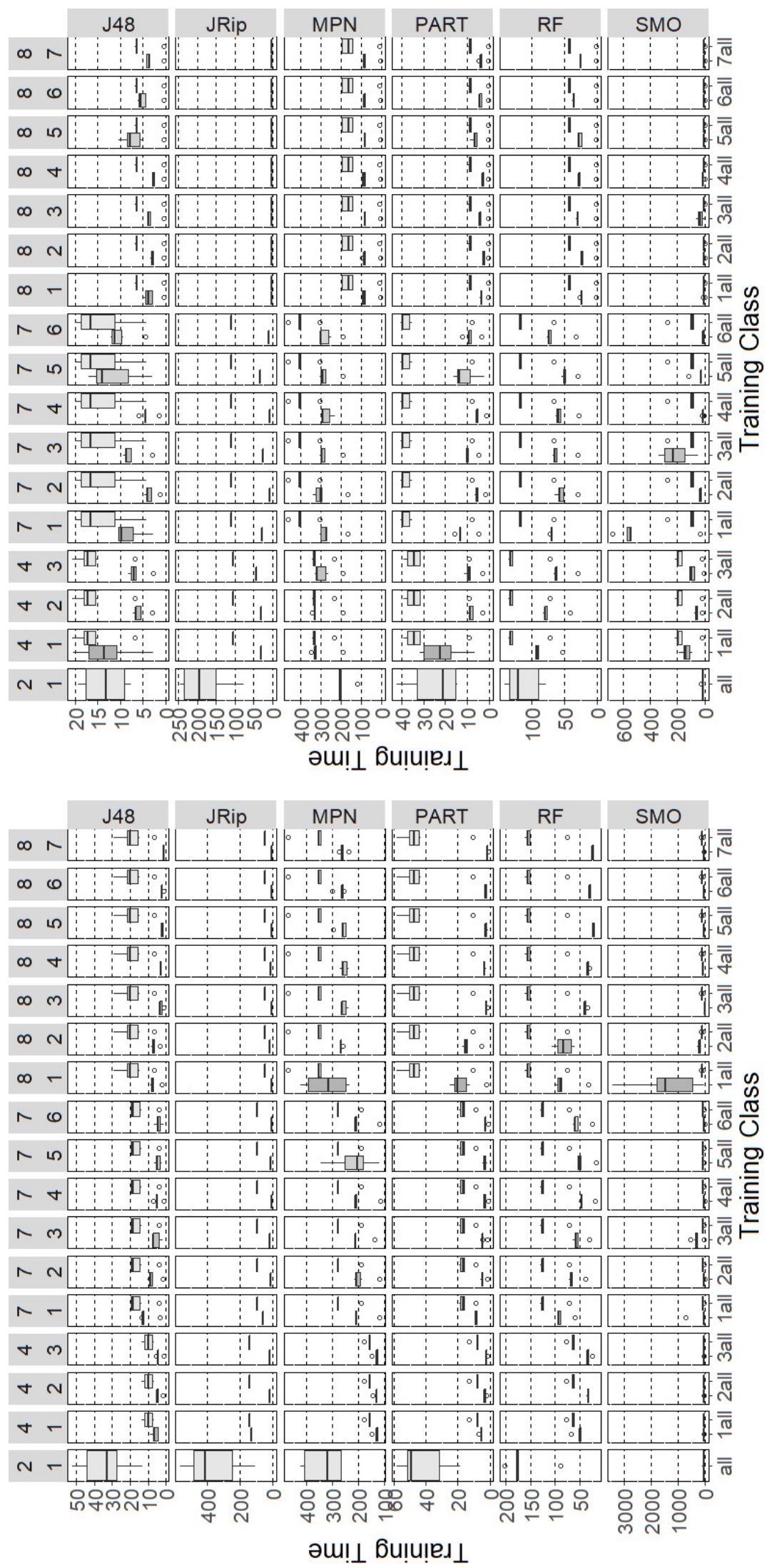


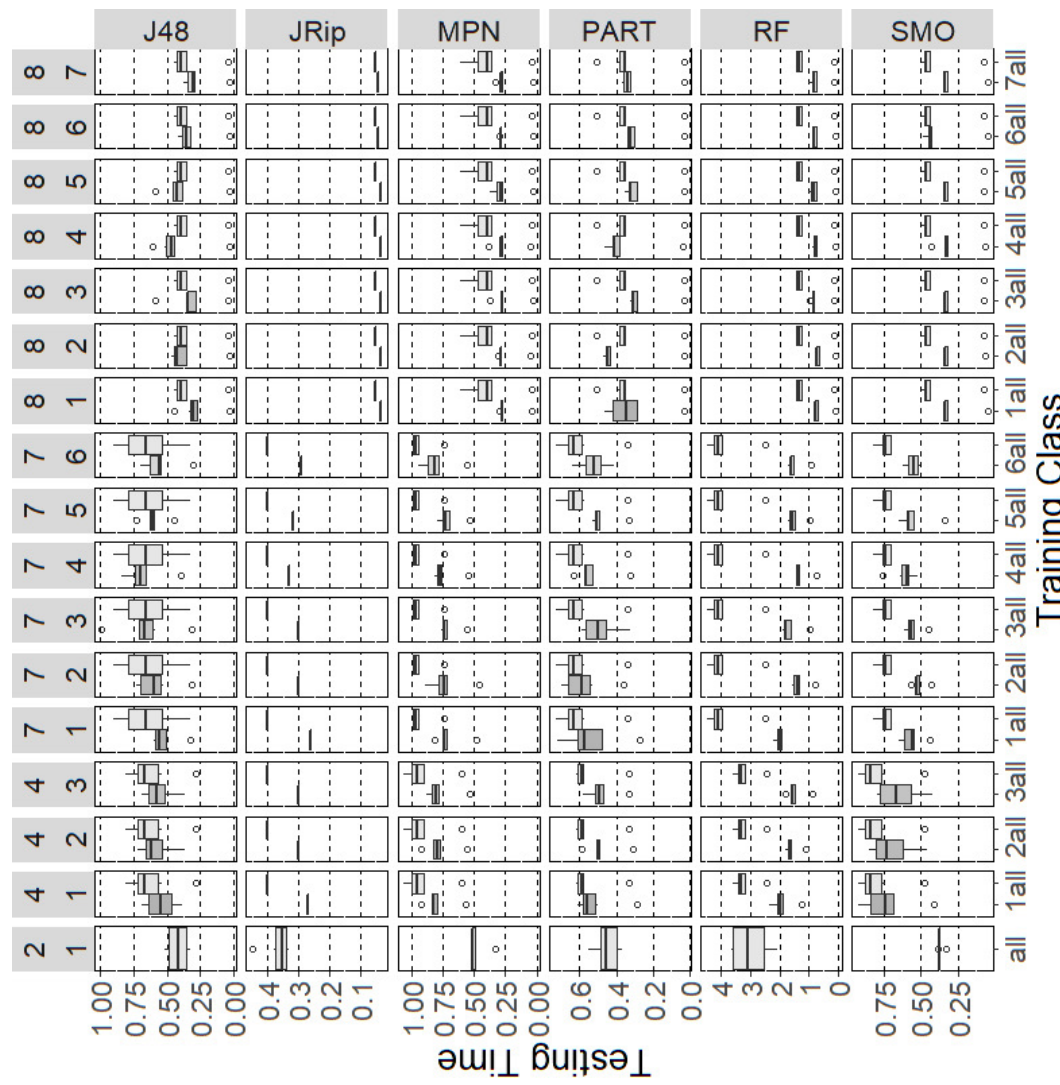

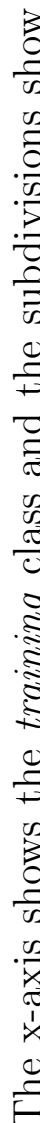

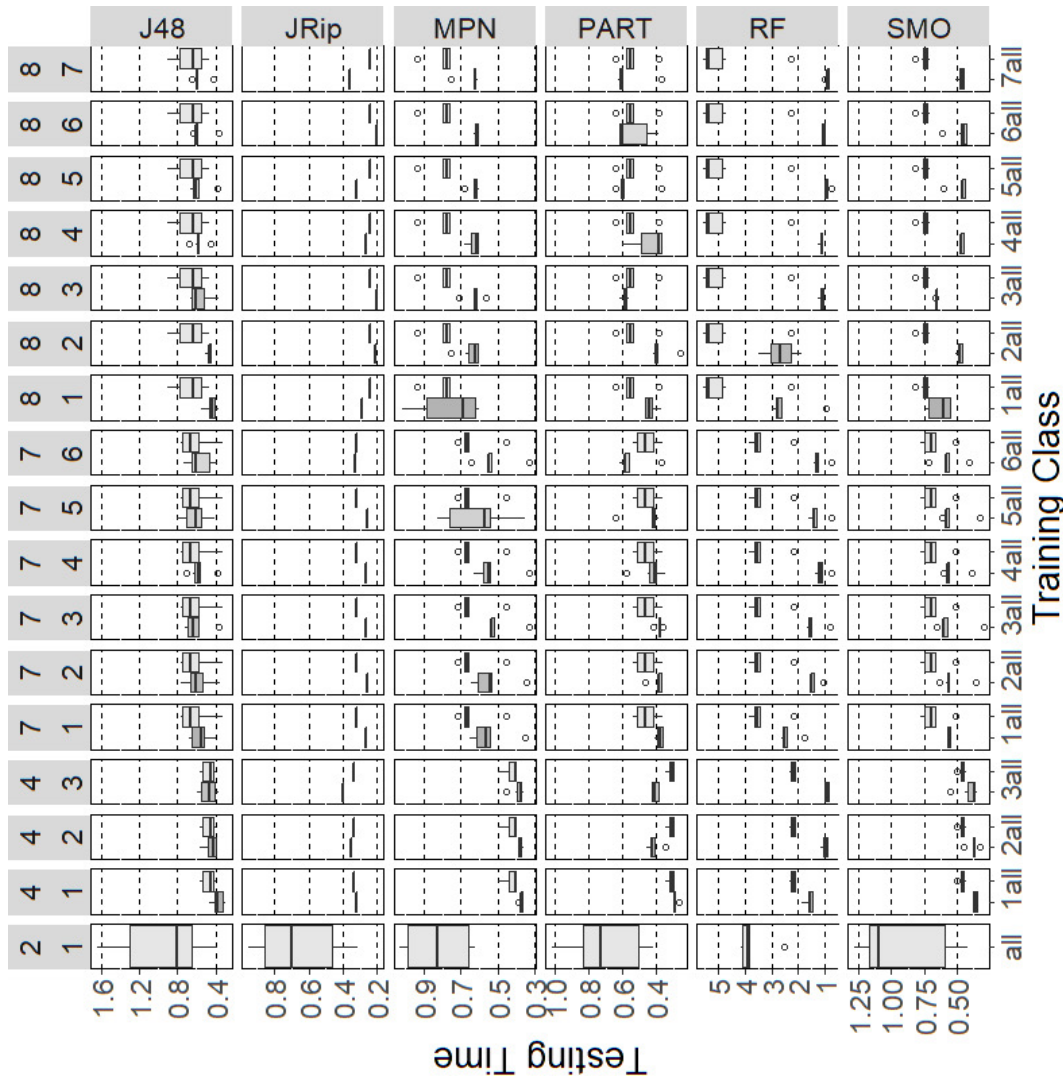



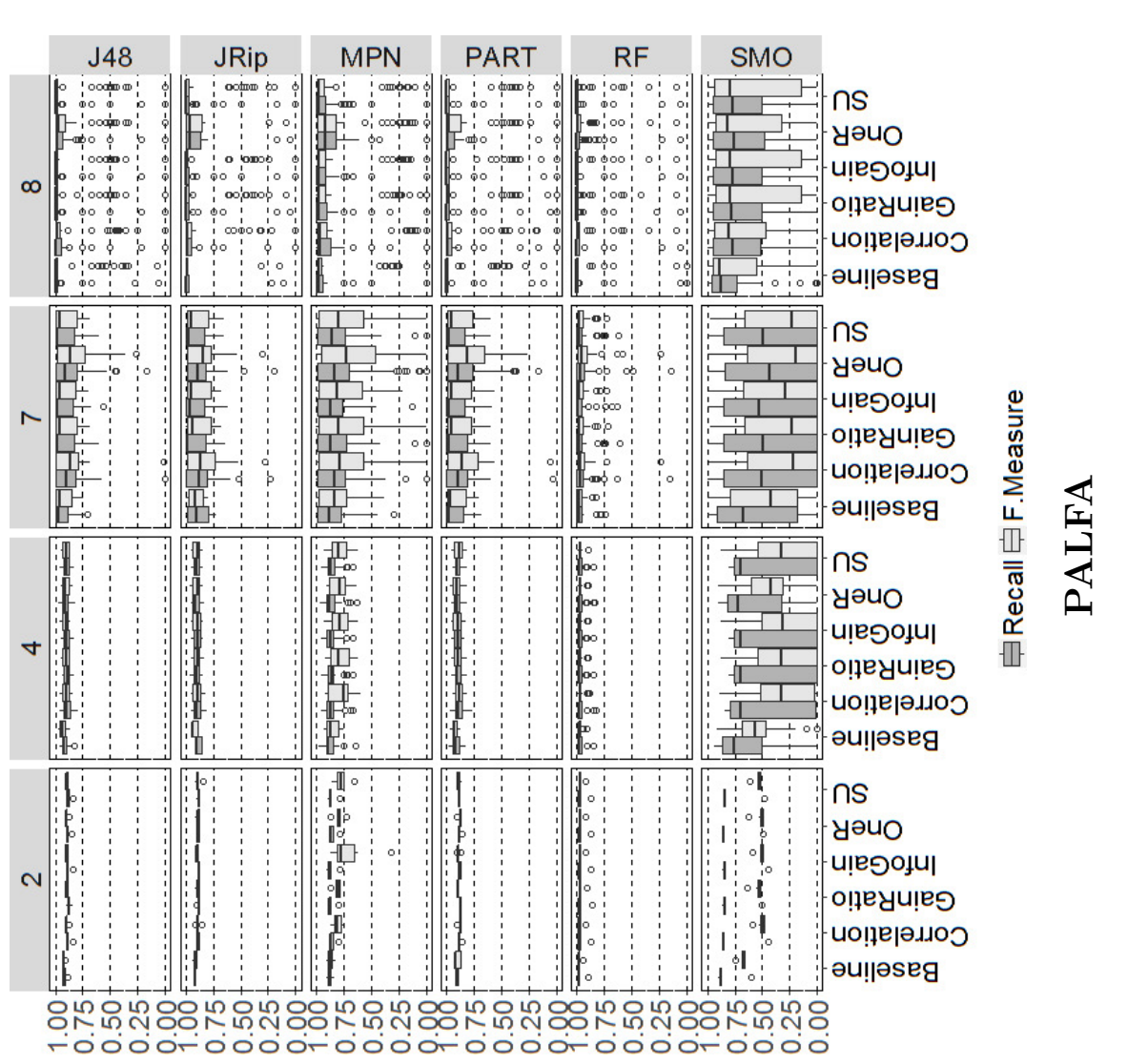

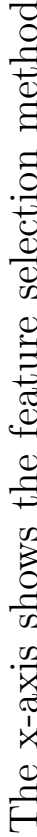
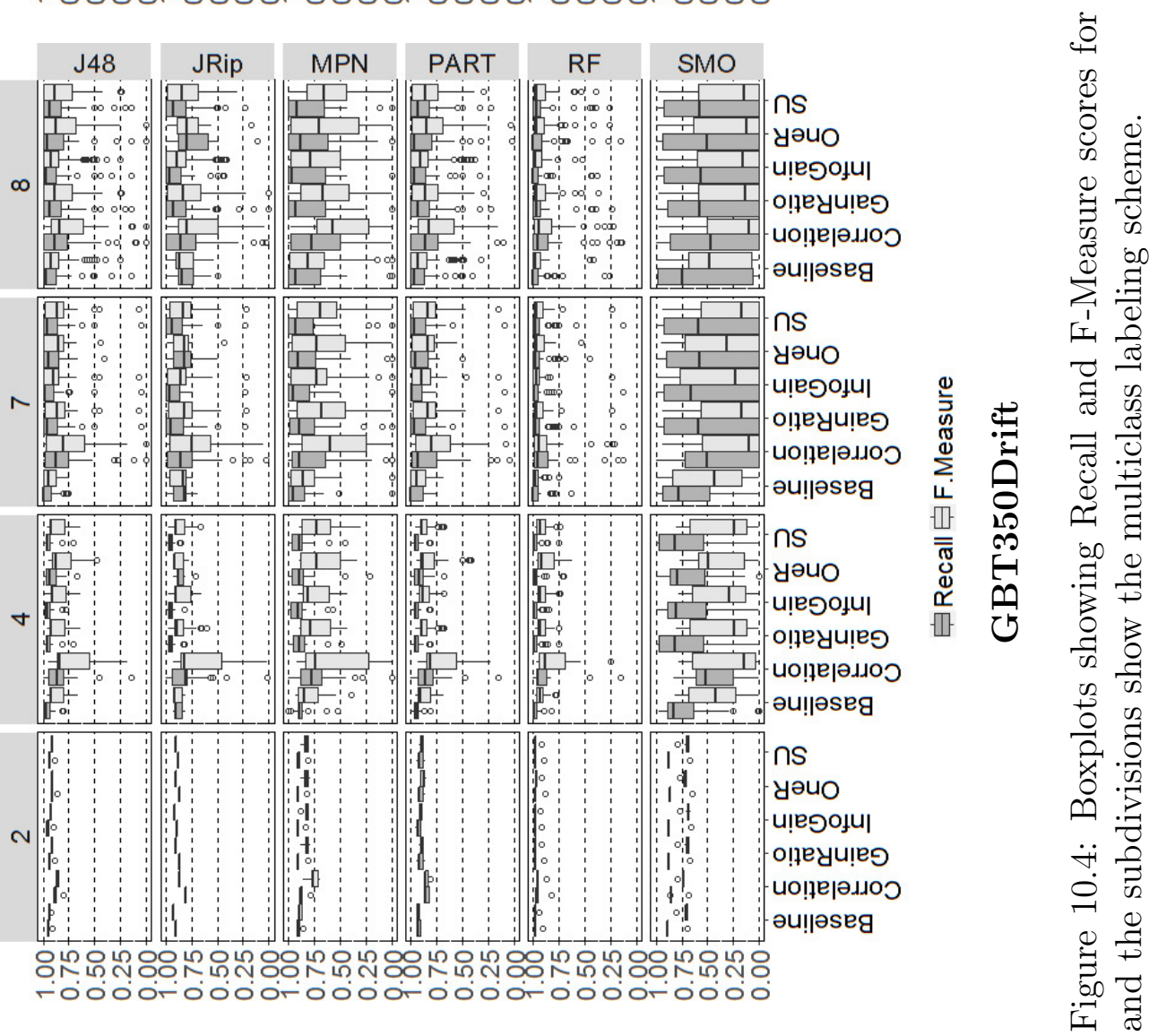

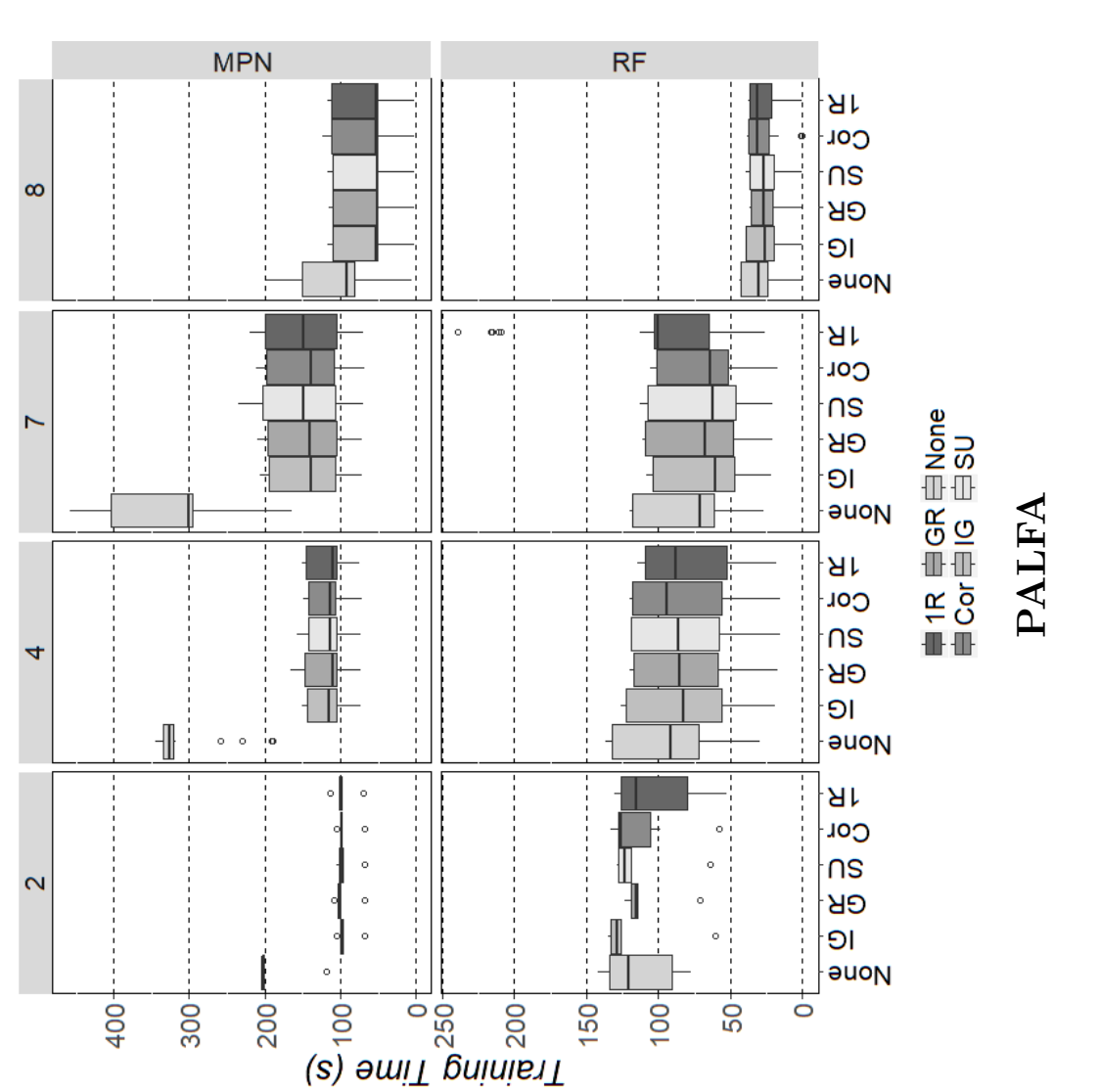

年
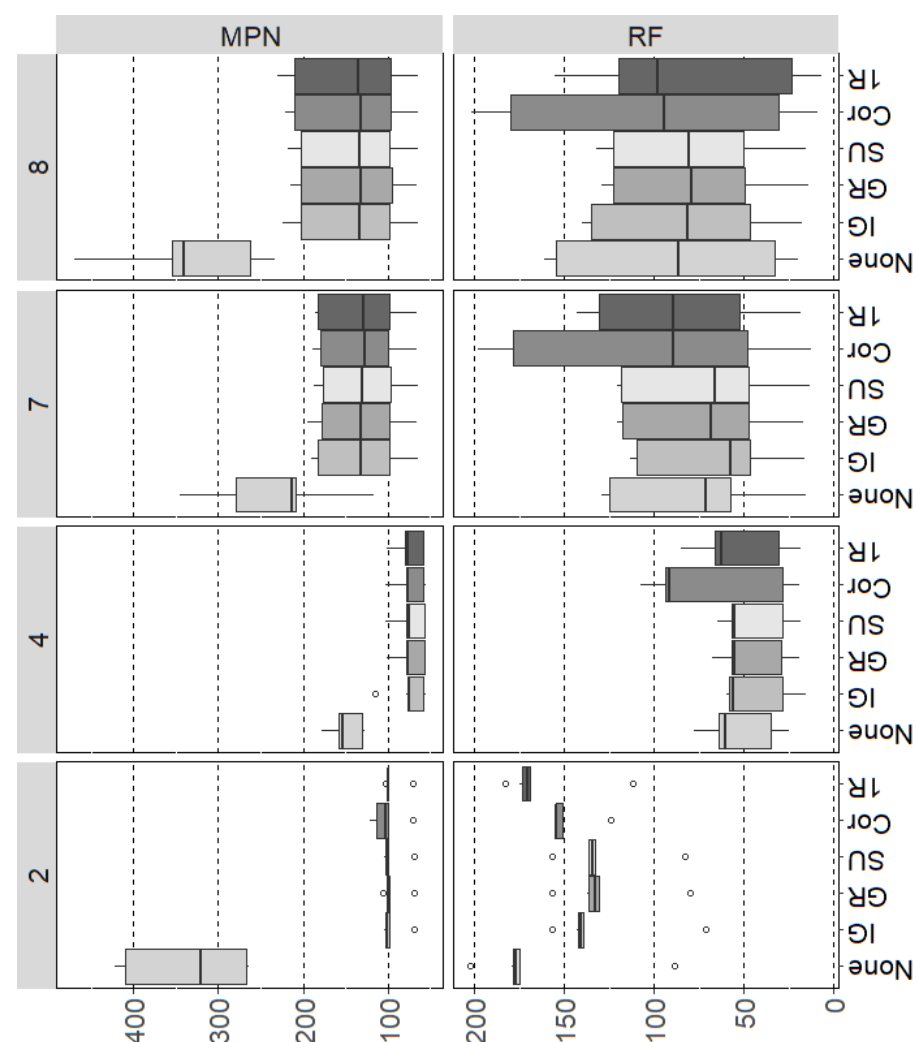

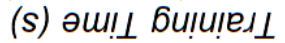

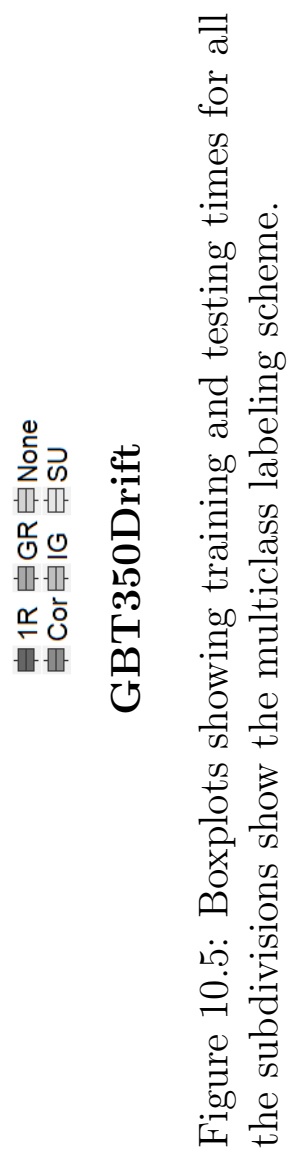



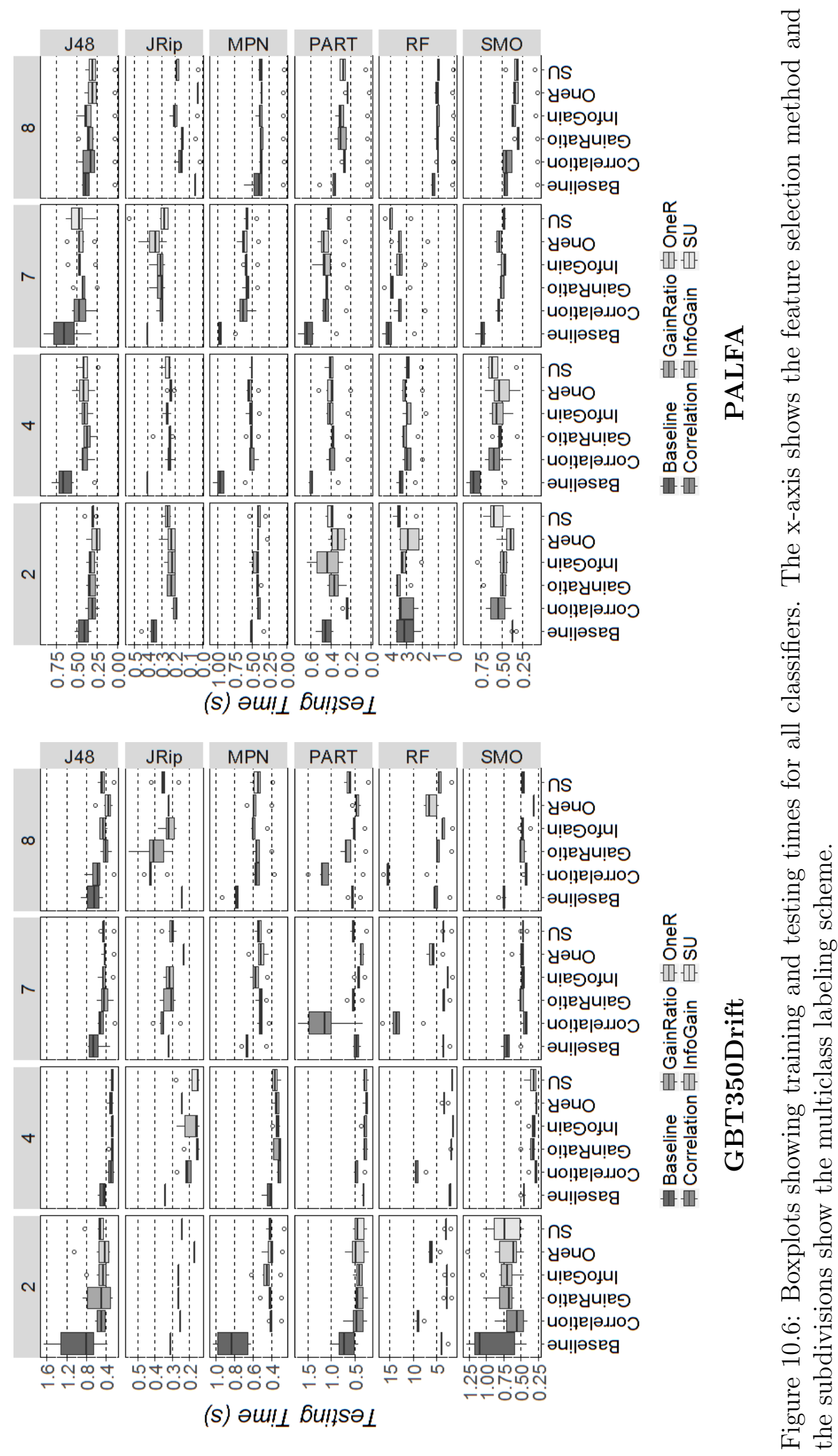


\section{References}

[1] A. Agresti and C. Franklin, Statistics: the art and science of learning from data, ser. MyStatLab Series. Pearson Prentice Hall, 2009.

[2] A. M. Aji et al., "On the efficacy of gpu-integrated mpi for scientific applications," in Proceedings of the 22nd Int'l Symposium on High-performance Parallel and Distributed Computing, ser. HPDC '13. New York, NY, USA: ACM, 2013, pp. 191-202.

[3] G. Allen et al., "Enabling applications on the grid: A gridlab overview," Int'l Journal on High Performance Computing Applications, vol. 17, no. 4, pp. 449-466, Nov. 2003.

[4] D. Amaratunga, J. Cabrera, and Y.-S. Lee, "Enriched random forests," Bioinformatics, vol. 24, no. 18, pp. 2010-2014, Sep. 2008. [Online]. Available: http://dx.doi.org/10.1093/bioinformatics/btn356

[5] M. Bagchi, D. R. Lorimer, and J. Chennamangalam, "Luminosities of recycled radio pulsars in globular clusters," MNRAS, vol. 418, pp. 477-489, Nov. 2011.

[6] E. D. Barr, D. J. Champion, M. Kramer, R. P. Eatough, P. C. C. Freire, R. Karuppusamy, K. J. Lee, J. P. W. Verbiest, C. G. Bassa, A. G. Lyne, B. Stappers, D. R. Lorimer, and B. Klein, "The Northern High Time Resolution Universe pulsar survey - I. Setup and initial discoveries," Monthly Notices of the Royal Astronomical Society, vol. 435, no. 3, pp. 2234-2245, 09 2013. [Online]. Available: https://doi.org/10.1093/mnras/stt1440

[7] S. D. Bates, M. Bailes, B. R. Barsdell, N. D. R. Bhat, M. Burgay, S. Burke-Spolaor, D. J. Champion, P. Coster, N. D'Amico, A. Jameson, S. Johnston, M. J. Keith, M. Kramer, L. Levin, A. Lyne, S. Milia, C. Ng, C. Nietner, A. Possenti, B. Stappers, D. Thornton, and W. van Straten, "The High Time Resolution Universe Pulsar Survey - VI. An artificial neural network and timing of 75 pulsars," Monthly Notices of the Royal Astronomical Society, vol. 427, pp. 1052-1065, Dec. 2012.

[8] Y. Bengio, J. Louradour, R. Collobert, and J. Weston, "Curriculum learning," in Proceedings of the 26th Annual International Conference on Machine Learning, ser. ICML '09. New York, NY, USA: Association for Computing Machinery, 2009, p. 41-48. [Online]. Available: https://doi.org/10.1145/1553374.1553380 
[9] S. Bernard, S. Adam, and L. Heutte, "Dynamic random forests," Pattern Recognition Letters, vol. 33, no. 12, pp. 1580 - 1586, 2012. [Online]. Available: http://www.sciencedirect.com/science/article/pii/S0167865512001274

[10] S. Bethapudi and S. Desai, "Separation of pulsar signals from noise using supervised machine learning algorithms," Astronomy and Computing, vol. 23, p. 15-26, Apr 2018. [Online]. Available: http://dx.doi.org/10.1016/j.ascom.2018.02.002

[11] G. Biau and E. Scornet, "A random forest guided tour," TEST, vol. 25, no. 2, pp. 197-227, Jun 2016. [Online]. Available: https://doi.org/10.1007/s11749-016-0481-7

[12] C. M. Bishop, Neural Networks for Pattern Recognition. Oxford University Press, 1995.

[13] A. Blum and T. Mitchell, "Combining labeled and unlabeled data with co-training," in Proceedings of the Eleventh Annual Conference on Computational Learning Theory, ser. COLT' 98. New York, NY, USA: ACM, 1998, pp. 92-100. [Online]. Available: http://doi.acm.org.www.libproxy.wvu.edu/10.1145/279943.279962

[14] A. L. Blum and P. Langley, "Selection of relevant features and examples in machine learning," Artificial Intelligence, vol. 97, no. 1, pp. 245 - 271, 1997, relevance. [Online]. Available: http://www.sciencedirect.com/science/article/pii/S0004370297000635

[15] J. Boyles, R. S. Lynch, S. M. Ransom, I. H. Stairs, D. R. Lorimer, M. A. McLaughlin, J. W. T. Hessels, V. M. Kaspi, V. I. Kondratiev, A. Archibald, A. Berndsen, R. F. Cardoso, A. Cherry, C. R. Epstein, C. Karako-Argaman, C. A. McPhee, T. Pennucci, M. S. E. Roberts, K. Stovall, and J. van Leeuwen, "The Green Bank Telescope 350 $\mathrm{MHz}$ Drift-scan survey. I. Survey Observations and the Discovery of 13 Pulsars," The Astrophysical Journal, vol. 763, p. 80, Feb. 2013.

[16] L. Breiman, J. Friedman, R. Olshen, and C. Stone, Classification and Regression Trees. Monterey, CA: Wadsworth and Brooks, 1984.

[17] L. Breiman, "Bagging predictors," Mach. Learn., vol. 24, no. 2, p. 123-140, Aug. 1996. [Online]. Available: https://doi.org/10.1023/A:1018054314350

[18] —_, "Random forests," Machine Learning, vol. 45, no. 1, pp. 5-32, 2001.

[19] B. D. Bue et al., "Astronomical data triage for rapid science return," in Proceedings 2014 Conference on Big Data from Space (BiDS14), vol. 206, 2014.

[20] S. Burke-Spolaor, M. Bailes, S. Johnston, S. D. Bates, N. D. R. Bhat, M. Burgay, N. D’Amico, A. Jameson, M. J. Keith, M. Kramer, L. Levin, S. Milia, A. Possenti, B. Stappers, and W. van Straten, "The high time resolution universe pulsar survey - iii. single-pulse searches and preliminary analysis," MNRAS, vol. 416, no. 4, pp. 2465-2476, 2011. [Online]. Available: http://mnras.oxfordjournals.org/content/416/4/2465.abstract 
[21] P. S. Chakraborty, "Detection of pulsars using machine learning algoriothms - a study," in Emerging Trends in Artificial Intelligence for Internet of Things, December 2019, pp. 209-221.

[22] V. T. N. Chau and N. H. Phung, "A random forest-based self-training algorithm for study status prediction at the program level: minsemi-rf," in Multi-disciplinary Trends in Artificial Intelligence, C. Sombattheera, F. Stolzenburg, F. Lin, and A. Nayak, Eds. Cham: Springer International Publishing, 2016, pp. 219-230.

[23] N. V. Chawla, K. W. Bowyer, L. O. Hall, and W. P. Kegelmeyer, "Smote: synthetic minority over-sampling technique," Journal of artificial intelligence research, vol. 16, no. 1, pp. 321-357, 2002.

[24] N. Chawla, "Data mining for imbalanced datasets: An overview," in Data Mining and Knowledge Discovery Handbook, O. Maimon and L. Rokach, Eds. Springer US, 2005, pp. 853-867.

[25] J. Chen, K. Li, Z. Tang, K. Bilal, S. Yu, C. Weng, and K. Li, "A parallel random forest algorithm for big data in a spark cloud computing environment," IEEE Trans. Parallel Distrib. Syst., vol. 28, no. 4, p. 919-933, Apr. 2017. [Online]. Available: https://doi.org/10.1109/TPDS.2016.2603511

[26] D. Cieslak and N. Chawla, "Learning decision trees for unbalanced data," in Machine Learning and Knowledge Discovery in Databases, ser. Lecture Notes in Computer Science, W. Daelemans, B. Goethals, and K. Morik, Eds. Springer Berlin Heidelberg, 2008, vol. 5211, pp. 241-256.

[27] W. W. Cohen, "Fast effective rule induction," in Twelfth International Conference on Machine Learning. Morgan Kaufmann, 1995, pp. 115-123.

[28] J. M. Cordes, P. C. C. Freire, D. R. Lorimer, F. Camilo, D. J. Champion, D. J. Nice, R. Ramachandran, J. W. T. Hessels, W. Vlemmings, J. van Leeuwen, S. M. Ransom, N. D. R. Bhat, Z. Arzoumanian, M. A. McLaughlin, V. M. Kaspi, L. Kasian, J. S. Deneva, B. Reid, S. Chatterjee, J. L. Han, D. C. Backer, I. H. Stairs, A. A. Deshpande, and C.-A. Faucher-Giguère, "Arecibo Pulsar Survey Using ALFA. I. Survey Strategy and First Discoveries," The Astrophysical Journal, vol. 637, pp. 446-455, Jan. 2006.

[29] J. M. Cordes and M. A. McLaughlin, "Searches for Fast Radio Transients," The Astrophysical Journal, vol. 596, pp. 1142-1154, Oct. 2003.

[30] D. D'Agostino et al., "The drihm project: A flexible approach to integrate hpc, grid and cloud resources for hydro-meteorological research," in Proceedings of the Int'l Conference for High Performance Computing, Networking, Storage and Analysis, ser. SC '14. Piscataway, NJ, USA: IEEE Press, 2014, pp. 536-546.

[31] D. B. Davidson, "Meerkat and ska phase 1," in ISAPE2012, Oct 2012, pp. 1279-1282. 
[32] J. S. Deneva, J. M. Cordes, M. A. McLaughlin, D. J. Nice, D. R. Lorimer, F. Crawford, N. D. R. Bhat, F. Camilo, D. J. Champion, P. C. C. Freire, S. Edel, V. I. Kondratiev, J. W. T. Hessels, F. A. Jenet, L. Kasian, V. M. Kaspi, M. Kramer, P. Lazarus, S. M. Ransom, I. H. Stairs, B. W. Stappers, J. van Leeuwen, A. Brazier, A. Venkataraman, J. A. Zollweg, and S. Bogdanov, "Arecibo Pulsar Survey Using ALFA: Probing Radio Pulsar Intermittency And Transients," The Astrophysical Journal, vol. 703, pp. 22592274, Oct. 2009.

[33] H. Deng and G. Runger, "Feature selection via regularized trees," The 2012 International Joint Conference on Neural Networks (IJCNN), 2012.

[34] T. R. Devine, K. Goseva-Popstojanova, and M. McLaughlin, "Detection of dispersed radio pulses: a machine learning approach to candidate identification and classification," Monthly Notices of the Royal Astronomical Society, vol. 459, pp. 1519-1532, Jun. 2016.

[35] T. R. Devine, K. Goseva-Popstojanova, and D. Pang, "Scalable solutions for automated single pulse identification and classification in radio astronomy," in Proceedings of the 47th International Conference on Parallel Processing, ser. ICPP 2018. New York, NY, USA: ACM, 2018, pp. 11:1-11:11. [Online]. Available: http://doi.acm.org/10.1145/3225058.3225101

[36] T. Dietterich, M. Kearns, and Y. Mansour, "Applying the weak learning framework to understand and improve c4.5," in In Proceedings of the Thirteenth International Conference on Machine Learning. Morgan Kaufmann, 1996, pp. 96-104.

[37] P. Domingos and G. Hulten, "Mining high-speed data streams," in Proceedings of the Sixth ACM SIGKDD International Conference on Knowledge Discovery and Data Mining, ser. KDD '00. New York, NY, USA: ACM, 2000, pp. 71-80. [Online]. Available: http://doi.acm.org.www.libproxy.wvu.edu/10.1145/347090.347107

[38] K. Driessens, P. Reutemann, B. Pfahringer, and C. Leschi, "Using weighted nearest neighbor to benefit from unlabeled data," in Advances in Knowledge Discovery and Data Mining, W.-K. Ng, M. Kitsuregawa, J. Li, and K. Chang, Eds. Berlin, Heidelberg: Springer Berlin Heidelberg, 2006, pp. 60-69.

[39] P. Du, W. A. Kibbe, and S. M. Lin, "Improved peak detection in mass spectrum by incorporating continuous wavelet transform-based pattern matching," Bioinformatics, vol. 22, no. 17, pp. 2059-2065, 2006.

[40] R. P. Eatough, E. F. Keane, and A. G. Lyne, "An interference removal technique for radio pulsar searches," MNRAS, vol. 395, no. 1, pp. 410-415, 2009. [Online]. Available: http://dx.doi.org/10.1111/j.1365-2966.2009.14524.x

[41] R. P. Eatough, N. Molkenthin, M. Kramer, A. Noutsos, M. J. Keith, B. W. Stappers, and A. G. Lyne, "Selection of radio pulsar candidates using artificial neural networks," MNRAS, vol. 407, pp. 2443-2450, Oct. 2010. 
[42] M. Ester, H.-P. Kriegel, J. Sander, and X. Xu, "A density-based algorithm for discovering clusters in large spatial databases with noise," in Proceedings of the Second International Conference on Knowledge Discovery and Data Mining, ser. KDD'96. AAAI Press, 1996, p. 226-231.

[43] C.-A. Faucher-Giguère and V. M. Kaspi, "Birth and Evolution of Isolated Radio Pulsars," The Astrophysical Journal, vol. 643, pp. 332-355, May 2006.

[44] A. J. Faulkner, I. H. Stairs, M. Kramer, A. G. Lyne, G. Hobbs, A. Possenti, D. R. Lorimer, R. N. Manchester, M. A. McLaughlin, N. D'Amico, F. Camilo, and M. Burgay, "The Parkes Multibeam Pulsar Survey - V. Finding binary and millisecond pulsars," Monthly Notices of the Royal Astronomical Society, vol. 355, pp. 147-158, Nov. 2004.

[45] E. Frank and I. H. Witten, "Generating accurate rule sets without global optimization," in Fifteenth International Conference on Machine Learning, J. Shavlik, Ed. Morgan Kaufmann, 1998, pp. 144-151.

[46] D. Frascarelli, S. Nesmachnow, and G. Tancredi, "High-performance computing of self-gravity for small solar system bodies," Computer, vol. 47, no. 9, pp. 34-39, Sept 2014.

[47] J. H. Friedman, "Greedy function approximation: A gradient boosting machine." Ann. Statist., vol. 29, no. 5, pp. 1189-1232, 10 2001. [Online]. Available: https://doi.org/10.1214/aos/1013203451

[48] C. Guidorzi, "MEPSA: A flexible peak search algorithm designed for uniformly spaced time series," Astronomy and Computing, vol. 10, pp. 54-60, Apr. 2015.

[49] P. Guo, F. Duan, P. Wang, Y. Yao, Q. Yin, X. Xin, D. Li, L. Qian, S. Wang, Z. Pan, and L. Zhang, "Pulsar candidate classification using generative adversary networks," Monthly Notices of the Royal Astronomical Society, vol. 490, no. 4, pp. 5424-5439, 11 2019. [Online]. Available: https://doi.org/10.1093/mnras/stz2975

[50] M. A. Hall and G. Holmes, "Benchmarking attribute selection techniques for discrete class data mining," IEEE Transactions on Knowledge and Data Engineering, vol. 15, no. 6, pp. 1437-1447, Nov 2003.

[51] M. Hall, E. Frank, G. Holmes, B. Pfahringer, P. Reutemann, and I. H. Witten, "The WEKA Data Mining Software: An Update," SIGKDD Explorations, vol. 11, 2009.

[52] M. A. Hall, "Correlation-based feature selection for machine learning," Master's thesis, University of Waikato, 1998.

[53] J. Han, M. Kamber, and J. Pei, Data Mining: Concepts and Techniques, 3rd ed. San Francisco, CA, USA: Morgan Kaufmann Publishers Inc., 2011.

[54] K. Harmer, G. Howells, W. Sheng, M. Fairhurst, and F. Deravi, "A peak-trough detection algorithm based on momentum," in Image and Signal Processing, 2008. CISP '08. Congress on, vol. 4, May 2008, pp. 454-458. 
[55] A. F. Hart, L. Cinquini, S. E. Khudikyan, D. R. Thompson, C. A. Mattmann, K. Wagstaff, J. Lazio, and D. L. Jones, "Supporting distributed, collaborative review and classification of fast transient events," COMPUTER, vol. 47, no. 9, pp. 65-66, 2014.

[56] A. Hewish, S. J. Bell, J. D. H. Pilkington, P. F. Scott, and R. A. Collins, "Observation of a rapidly pulsating radio source," Nature, vol. 217, pp. 709-713, Feb. 1968.

[57] W. Hua, S. Wang, H. Liu, K. Liu, Y. Guo, and L. Jiao, "Semisupervised polsar image classification based on improved cotraining," IEEE Journal of Selected Topics in Applied Earth Observations and Remote Sensing, vol. 10, no. 11, pp. 4971-4986, Nov 2017.

[58] M. Jenne et al., "Studying the milky way galaxy using paraheap-k," Computer, vol. 47, no. 9, pp. 26-33, Sept 2014.

[59] G. H. John, R. Kohavi, and K. Pfleger, "Irrelevant features and the subset selection problem," in Proceedings of the Eleventh International Conference on International Conference on Machine Learning, ser. ICML'94. San Francisco, CA, USA: Morgan Kaufmann Publishers Inc., 1994, pp. 121-129. [Online]. Available: http://dl.acm.org/citation.cfm?id=3091574.3091590

[60] D. Jones, K. Wagstaff, D. Thompson, L. D'Addario, R. Navarro, C. Mattmann, W. Majid, J. Lazio, R. Preston, and U. Rebbapragada, "Big data challenges for large radio arrays," in Aerospace Conference, 2012 IEEE, March 2012, pp. 1-6.

[61] R. Jongerius, S. Wijnholds, R. Nijboer, and H. Corporaal, "An end-to-end computing model for the square kilometre array," Computer, vol. 47, no. 9, pp. 48-54, 2014.

[62] C. Karako-Argaman, V. M. Kaspi, R. S. Lynch, J. W. T. Hessels, V. I. Kondratiev, M. A. McLaughlin, S. M. Ransom, A. M. Archibald, J. Boyles, F. A. Jenet, D. L. Kaplan, L. Levin, D. R. Lorimer, E. C. Madsen, M. S. E. Roberts, X. Siemens, I. H. Stairs, K. Stovall, J. K. Swiggum, and J. van Leeuwen, "Discovery and Follow-up of Rotating Radio Transients with the Green Bank and LOFAR Telescopes," The Astrophysical Journal, vol. 809, p. 67, Aug. 2015.

[63] H. Karau and R. Warren, High Performance Spark:Best practices for scaling and optimizing Apache Spark. USA: O'Reilly Media, Inc, 2016.

[64] A. G. Karegowda, A. S. Manjunath, and M. A. Jayaram, "Comparative study of attribute selection using gain ratio and correlation based feature selection," International Journal of Information Technology and Knowledge Management, vol. 2, no. 2, pp. 271-277, 2010.

[65] E. F. Keane, D. A. Ludovici, R. P. Eatough, M. Kramer, A. G. Lyne, M. A. McLaughlin, and B. W. Stappers, "Further searches for Rotating Radio Transients in the Parkes Multi-beam Pulsar Survey," MNRAS, vol. 401, pp. 1057-1068, Jan. 2010. 
[66] S. Keerthi, S. Shevade, C. Bhattacharyya, and K. Murthy, "Improvements to platt's smo algorithm for svm classifier design," Neural Computation, vol. 13, no. 3, pp. 637649, 2001.

[67] M. J. Keith, R. P. Eatough, A. G. Lyne, M. Kramer, A. Possenti, F. Camilo, and R. N. Manchester, "Discovery of 28 pulsars using new techniques for sorting pulsar candidates," Monthly Notices of the Royal Astronomical Society, vol. 395, pp. 837846, May 2009.

[68] M. J. Keith, A. Jameson, W. van Straten, M. Bailes, S. Johnston, M. Kramer, A. Possenti, S. D. Bates, N. D. R. Bhat, M. Burgay, S. Burke-Spolaor, N. D’Amico, L. Levin, P. L. McMahon, S. Milia, and B. W. Stappers, "The High Time Resolution Universe Pulsar Survey - I. System configuration and initial discoveries," Monthly Notices of the Royal Astronomical Society, vol. 409, pp. 619-627, Dec. 2010.

[69] W. Kirch, Ed., Pearson's Correlation Coefficient. Dordrecht: Springer Netherlands, 2008, pp. 1090-1091.

[70] R. Kohavi and G. H. John, "Wrappers for feature subset selection," Artificial intelligence, vol. 97, no. 1-2, pp. 273-324, 1997.

[71] E. Konukoglu and M. Ganz, "Approximate false positive rate control in selection frequency for random forest," CoRR, vol. abs/1410.2838, 2014. [Online]. Available: http://arxiv.org/abs/1410.2838

[72] M. P. Kumar, B. Packer, and D. Koller, "Self-paced learning for latent variable models," in Advances in Neural Information Processing Systems 23, J. D. Lafferty, C. K. I. Williams, J. Shawe-Taylor, R. S. Zemel, and A. Culotta, Eds. Curran Associates, Inc., 2010, pp. 1189-1197. [Online]. Available: http://papers.nips.cc/paper/3923-self-paced-learning-for-latent-variable-models.pdf

[73] S. Larsson, "Parameter estimation in epoch folding analysis." Astronomy and Astrophysics, Supplement, vol. 117, pp. 197-201, May 1996.

[74] P. Lazarus, A. Brazier, J. Hessels, C. Karako-Argaman, V. Kaspi, R. Lynch, E. Madsen, C. Patel, S. Ransom, P. Scholz, J. Swiggum, W. Zhu, B. Allen, S. Bogdanov, F. Camilo, F. Cardoso, S. Chatterjee, J. Cordes, F. Crawford, and A. Venkataraman, "Arecibo pulsar survey using alfa. iv. mock spectrometer data analysis, survey sensitivity, and the discovery of 41 pulsars," The Astrophysical Journal, vol. 812, 042015.

[75] K. J. Lee, K. Stovall, F. A. Jenet, J. Martinez, L. P. Dartez, A. Mata, G. Lunsford, S. Cohen, C. M. Biwer, M. Rohr, J. Flanigan, A. Walker, S. Banaszak, B. Allen, E. D. Barr, N. D. R. Bhat, S. Bogdanov, A. Brazier, F. Camilo, D. J. Champion, S. Chatterjee, J. Cordes, F. Crawford, J. Deneva, G. Desvignes, R. D. Ferdman, P. Freire, J. W. T. Hessels, R. Karuppusamy, V. M. Kaspi, B. Knispel, M. Kramer, P. Lazarus, R. Lynch, A. Lyne, M. McLaughlin, S. Ransom, P. Scholz, X. Siemens, L. Spitler, I. Stairs, M. Tan, J. van Leeuwen, and W. W. Zhu, "PEACE: pulsar evaluation algorithm for candidate extraction - a software package for post-analysis processing of 
pulsar survey candidates," Monthly Notices of the Royal Astronomical Society, vol. 433, pp. 688-694, Jul. 2013.

[76] C. Leistner, A. Saffari, J. Santner, and H. Bischof, "Semi-supervised random forests," in 2009 IEEE 12th International Conference on Computer Vision, Sep. 2009, pp. 506513.

[77] H. Li and E. E. Fenimore, "Log-normal Distributions in Gamma-Ray Burst Time Histories," The Astrophysical Journall, vol. 469, p. L115, Oct. 1996.

[78] M. Li and Z.-H. Zhou, "Improve computer-aided diagnosis with machine learning techniques using undiagnosed samples," Systems, Man and Cybernetics, Part A: Systems and Humans, IEEE Transactions on, vol. 37, pp. 1088 - 1098, 122007.

[79] C. Liang, Y. Zhang, P. Shi, and Z. Hu, "Learning very fast decision tree from uncertain data streams with positive and unlabeled samples," Inf. Sci., vol. 213, pp. 50-67, Dec. 2012. [Online]. Available: https://doi.org/10.1016/j.ins.2012.05.023

[80] C. X. Ling, J. Du, and Z.-H. Zhou, "When does co-training work in real data?" in Advances in Knowledge Discovery and Data Mining, T. Theeramunkong, B. Kijsirikul, N. Cercone, and T.-B. Ho, Eds. Berlin, Heidelberg: Springer Berlin Heidelberg, 2009, pp. 596-603.

[81] D. R. Lorimer and M. Kramer, Handbook of Pulsar Astronomy. Cambridge University Press, Oct. 2012.

[82] B. Ludäscher et al., "Scientific workflow management and the kepler system: Research articles," Concurrency and Computation : Practice and Experience, vol. 18, no. 10, pp. 1039-1065, Aug. 2006.

[83] R. J. Lyon, J. M. Brooke, J. D. Knowles, and B. W. Stappers, "A Study on Classification in Imbalanced and Partially-Labelled Data Streams," ArXiv e-prints, Jul. 2013.

[84] R. J. Lyon, B. W. Stappers, S. Cooper, J. M. Brooke, and J. D. Knowles, "Fifty years of pulsar candidate selection: from simple filters to a new principled real-time classification approach," Monthly Notices of the Royal Astronomical Society, vol. 459, no. 1, pp. 1104-1123, 04 2016. [Online]. Available: https://doi.org/10.1093/mnras/stw656

[85] F. Ma, D. Meng, Q. Xie, Z. Li, and X. Dong, "Self-paced co-training," in Proceedings of the 34th International Conference on Machine Learning Volume 70, ser. ICML'17. JMLR.org, 2017, pp. 2275-2284. [Online]. Available: http://dl.acm.org.www.libproxy.wvu.edu/citation.cfm?id=3305890.3305916

[86] Z. Ma, J. Zhu, Y. Zhu, and H. Xu, "Classification of radio galaxy images with semisupervised learning," in Data Mining and Big Data, Y. Tan and Y. Shi, Eds. Singapore: Springer Singapore, 2019, pp. 191-200. 
[87] R. N. Manchester, G. B. Hobbs, A. Teoh, and M. Hobbs, "The Australia Telescope National Facility Pulsar Catalogue," The Astrophysical Journal, vol. 129, pp. 1993 2006, Apr. 2005.

[88] R. N. Manchester, A. G. Lyne, F. Camilo, J. F. Bell, V. M. Kaspi, N. D'Amico, N. P. F. McKay, F. Crawford, I. H. Stairs, A. Possenti, M. Kramer, and D. C. Sheppard, "The Parkes multi-beam pulsar survey - I. Observing and data analysis systems, discovery and timing of 100 pulsars," Monthly Notices of the Royal Astronomical Society, vol. 328, pp. 17-35, Nov. 2001.

[89] M. A. McLaughlin, A. G. Lyne, D. R. Lorimer, M. Kramer, A. J. Faulkner, R. N. Manchester, J. M. Cordes, F. Camilo, A. Possenti, I. H. Stairs, G. Hobbs, N. D'Amico, M. Burgay, and J. T. O'Brien, "Transient radio bursts from rotating neutron stars," Nature, vol. 439, pp. 817-820, Feb. 2006.

[90] V. Morello, E. D. Barr, M. Bailes, C. M. Flynn, E. F. Keane, and W. van Straten, "SPINN: a straightforward machine learning solution to the pulsar candidate selection problem," MNRAS, vol. 443, pp. 1651-1662, Sep. 2014.

[91] S. Muller, G. Alonso, and A. Csillaghy, "Scaling astroinformatics: Python + automatic parallelization," Computer, vol. 47, no. 9, pp. 41-47, Sept 2014.

[92] R. Nan, D. Li, C. Jin, Q. Wang, L. Zhu, W. Zhu, H. Zhang, Y. Yue, and L. Qian, "The Five-Hundred Aperture Spherical Radio Telescope (fast) Project," International Journal of Modern Physics D, vol. 20, pp. 989-1024, 2011.

[93] K. Nigam and R. Ghani, "Analyzing the effectiveness and applicability of co-training," in Proceedings of the Ninth International Conference on Information and Knowledge Management, ser. CIKM '00. New York, NY, USA: ACM, 2000, pp. 86-93. [Online]. Available: http://doi.acm.org.www.libproxy.wvu.edu/10.1145/354756.354805

[94] M. Odersky, L. Spoon, and B. Venners, Programming in Scala: A Comprehensive Step-by-Step Guide, 2Nd Edition, 2nd ed. USA: Artima Incorporation, 2011.

[95] A. Orriols-Puig and E. Bernardo-Mansilla, "Evolutionary rule-based systems for imbalanced data sets," Soft Computing, vol. 13, no. 3, pp. 213-225, 2009. [Online]. Available: http://dx.doi.org/10.1007/s00500-008-0319-7

[96] G. K. Palshikar, "Simple Algorithms for Peak Detection in Time-Series," in Proc. 1st Int. Conf. Advanced Data Analysis, Business Analytics and Intelligence, 2009.

[97] D. Pang, K. Goseva-Popstojanova, T. Devine, and M. McLaughlin, "A novel single-pulse search approach to detection of dispersed radio pulses using clustering and supervised machine learning," Monthly Notices of the Royal Astronomical Society, p. sty1992, 2018. [Online]. Available: http://dx.doi.org/10.1093/mnras/sty1992

[98] V. Pankratius and C. Mattmann, "Computing in astronomy: To see the unseen," Computer, vol. 47, no. 9, pp. 23-25, Sept 2014. 
[99] B. Pfahringer, P. Reutemann, and M. Mayo, "A novel two stage scheme utilizing the test set for model selection in text classification," in Proc Workshop on Learning Algorithms for Pattern Recognition, Eighteenth Australian Joint Conference on Artificial Intelligence (AI'05), R. Ghosh, B. Verma, and X. Li, Eds. Sydney, Australia: University of Technology, 2005, pp. 60-65, 5-9 December 2005.

[100] J. Platt, "Fast training of support vector machines using sequential minimal optimization," in Advances in Kernel Methods - Support Vector Learning, B. Schoelkopf, C. Burges, and A. Smola, Eds. MIT Press, 1998. [Online]. Available: http://research.microsoft.com/ jplatt/smo.html

[101] J. Prakash and D. L. M. Nithya, "A survey on semi-supervised learning techniques," International Journal of Computer Trends and Technology, vol. 8, 022014.

[102] J. Qiu et al., "A survey of machine learning for big data processing," EURASIP Journal on Advances in Signal Processing, vol. 2016, no. 1, p. 67, May 2016.

[103] R. Quinlan, C4.5: Programs for Machine Learning. San Mateo, CA: Morgan Kaufmann Publishers, 1993.

[104] R. Ramakrishnan and J. Gehrke, Database Management Systems, 3rd ed. New York, NY, USA: McGraw-Hill, Inc., 2003.

[105] S. M. Ransom, "New search techniques for binary pulsars," Ph.D. dissertation, Harvard University, 2001.

[106] I. Reis and D. Baron, "PRF: Probabilistic Random Forest," Astrophysics Source Code Library, Mar. 2019.

[107] M. T. Ribeiro, S. Singh, and C. Guestrin, "Model-agnostic interpretability of machine learning," 2016.

[108] E. Rubio-Herrera, B. W. Stappers, J. W. T. Hessels, and R. Braun, "A search for radio pulsars and fast transients in M31 using the Westerbork Synthesis Radio Telescope," MNRAS, vol. 428, pp. 2857-2873, Feb. 2013.

[109] S. J. Russell and P. Norvig, Artificial Intelligence: A Modern Approach, 2nd ed. Pearson Education, 2003.

[110] Sanidas, S., Cooper, S., Bassa, C. G., Hessels, J. W. T., Kondratiev, V. I., Michilli, D., Stappers, B. W., Tan, C. M., van Leeuwen, J., Cerrigone, L., Fallows, R. A., Iacobelli, M., Orrú, E., Pizzo, R. F., Shulevski, A., Toribio, M. C., ter Veen, S., Zucca, P., Bondonneau, L., Grießmeier, J.-M., Karastergiou, A., Kramer, M., and Sobey, C., "The lofar tied-array all-sky survey (lotaas): Survey overview and initial pulsar discoveries," $A \mathscr{E} A$, vol. 626, p. A104, 2019. [Online]. Available: https://doi.org/10.1051/0004-6361/201935609 
[111] I. Santos, C. Laorden, and P. G. Bringas, "Collective classification for unknown malware detection," Proceedings of the International Conference on Security and Cryptography, pp. 251-256, 2011.

[112] R. E. Schapire, "A brief introduction to boosting," in Proceedings of the 16th International Joint Conference on Artificial Intelligence - Volume 2, ser. IJCAI'99. San Francisco, CA, USA: Morgan Kaufmann Publishers Inc., 1999, p. 1401-1406.

[113] S. Schulter, P. Wohlhart, C. Leistner, A. Saffari, P. M. Roth, and H. Bischof, "Alternating decision forests," in The IEEE Conference on Computer Vision and Pattern Recognition (CVPR), June 2013.

[114] P. Sen, G. M. Namata, M. Bilgic, L. Getoor, B. Gallagher, and T. Eliassi-Rad, "Collective classification in network data," AI Magazine, vol. 29, no. 3, pp. 93-106, 2008.

[115] Shaoqing Ren, X. Cao, Yichen Wei, and J. Sun, "Global refinement of random forest," in 2015 IEEE Conference on Computer Vision and Pattern Recognition (CVPR), June 2015, pp. 723-730.

[116] Y. Sun, M. S. Kamel, A. K. Wong, and Y. Wang, "Cost-sensitive boosting for classification of imbalanced data," Pattern Recognition, vol. 40, no. 12, pp. 3358 - 3378, 2007. [Online]. Available: http://www.sciencedirect.com/science/article/pii/ S0031320307001835

[117] A. Szalay, "Extreme data-intensive scientific computing," Computing in Science \&6 Engineering, vol. 13, pp. 34-41, 072011.

[118] C. Tan, R. Lyon, B. Stappers, S. Cooper, J. Hessels, V. Kondratiev, D. Michilli, and S. Sanidas, "Ensemble candidate classification for the lotaas pulsar survey," Royal Astronomical Society. Monthly Notices, vol. 474, no. 4, pp. 4571-4583, Mar. 2018.

[119] J. Tanha, M. van Someren, and H. Afsarmanesh, "Semi-supervised selftraining for decision tree classifiers," International Journal of Machine Learning and Cybernetics, vol. 8, no. 1, pp. 355-370, Feb 2017. [Online]. Available: https://doi.org/10.1007/s13042-015-0328-7

[120] M. L. Tong, Y. Zhang, W. Zhao, J. Z. Liu, C. S. Zhao, and T. G. Yang, "Using pulsar timing arrays and the quantum normalization condition to constrain relic gravitational waves," Classical and Quantum Gravity, vol. 31, no. 3, p. 035001, Feb. 2014.

[121] V. K. Vavilapalli, A. C. Murthy, C. Douglas, S. Agarwal, M. Konar, R. Evans, T. Graves, J. Lowe, H. Shah, S. Seth, B. Saha, C. Curino, O. O’Malley, S. Radia, B. Reed, and E. Baldeschwieler, "Apache hadoop yarn: Yet another resource negotiator," in Proceedings of the 4th Annual Symposium on Cloud Computing, ser. SOCC '13. New York, NY, USA: ACM, 2013, pp. 5:1-5:16. [Online]. Available: http://doi.acm.org/10.1145/2523616.2523633

[122] S. Wadkar and M. Siddalingaiah, Apache Ambari. Berkeley, CA: Apress, 2014, pp. 399-401. 
[123] S. Winham, R. Freimuth, and J. Biernacka, "A weighted random forests approach to improve predictive performance," Statistical Analysis and Data Mining, vol. 6, 122013.

[124] I. H. Witten and E. Frank, Data Mining: Practical Machine Learning Tools and Techniques, Second Edition. San Francisco, CA, USA: Morgan Kaufmann Publishers Inc., 2005.

[125] D. H. Wolpert, "The lack of a priori distinctions between learning algorithms," Neural Computation, vol. 8, no. 7, pp. 1341-1390, 1996.

[126] J. Wu, L. Li, and W. Y. Wang, "Reinforced co-training," CoRR, vol. abs/1804.06035, 2018. [Online]. Available: http://arxiv.org/abs/1804.06035

[127] X. Wu, P. Li, and X. Hu, "Learning from concept drifting data streams with unlabeled data," Neurocomput., vol. 92, pp. 145-155, Sep. 2012. [Online]. Available: http://dx.doi.org/10.1016/j.neucom.2011.08.041

[128] J. Xiao, X. Li, H. Lin, and K. Qiu, "Pulsar candidate selection using pseudo-nearest centroid neighbour classifier," Monthly Notices of the Royal Astronomical Society, vol. 492, no. 2, pp. 2119-2127, 12 2019. [Online]. Available: https://doi.org/10.1093/mnras/stz3539

[129] Y. Xing, G. Yu, C. Domeniconi, J. Wang, and Z. Zhang, "Multi-label cotraining," in Proceedings of the 27th International Joint Conference on Artificial Intelligence, ser. IJCAI'18. AAAI Press, 2018, pp. 2882-2888. [Online]. Available: http://dl.acm.org.www.libproxy.wvu.edu/citation.cfm?id=3304889.3305061

[130] M. Zaharia, M. Chowdhury, M. J. Franklin, S. Shenker, and I. Stoica, "Spark: Cluster computing with working sets," in Proceedings of the 2Nd USENIX Conference on Hot Topics in Cloud Computing, ser. HotCloud'10. Berkeley, CA, USA: USENIX Association, 2010, pp. 10-10. [Online]. Available: http://dl.acm.org/citation.cfm?id=1863103.1863113

[131] W. Zhan and M.-L. Zhang, "Inductive semi-supervised multi-label learning with co-training," in Proceedings of the 23rd ACM SIGKDD International Conference on Knowledge Discovery and Data Mining, ser. KDD '17. New York, NY, USA: ACM, 2017, pp. 1305-1314. [Online]. Available: http: //doi.acm.org.www.libproxy.wvu.edu/10.1145/3097983.3098141

[132] H. Zhang, Z. Zhao, T. An, B. Lao, and X. Chen, "Pulsar candidate recognition with deep learning," Computers \& Electrical Engineering, vol. 73, pp. 1 - 8, 2019. [Online]. Available: http://www.sciencedirect.com/science/article/pii/S004579061832127X

[133] J. Zhang, Y. Zhang, and Y. Zhao, "RR lyrae star candidates from SDSS databases by cost-sensitive random forests," The Astrophysical Journal Supplement Series, vol. 246, no. 1, p. 8, jan 2020. [Online]. Available: https://doi.org/10.3847/1538-4365/ab5a7c 
[134] Z. Zhang, K. Barbary, F. A. Nothaft, E. Sparks, O. Zahn, M. J. Franklin, D. A. Patterson, and S. Perlmutter, "Scientific computing meets big data technology: An astronomy use case," in Proceedings of the 2015 IEEE International Conference on Big Data (Big Data), ser. BIG DATA '15. Washington, DC, USA: IEEE Computer Society, 2015, pp. 918-927. [Online]. Available: https://doi.org/10.1109/BigData.2015.7363840

[135] Z.-H. Zhou and M. Li, "Tri-training: Exploiting unlabeled data using three classifiers," IEEE Trans. on Knowl. and Data Eng., vol. 17, no. 11, p. 1529-1541, Nov. 2005. [Online]. Available: https://doi.org/10.1109/TKDE.2005.186

[136] R. Zhu, D. Zeng, and M. Kosorok, "Reinforcement learning trees," Journal of the American Statistical Association, vol. 110, pp. 0-0, 042015.

[137] W. W. Zhu, A. Berndsen, E. C. Madsen, M. Tan, I. H. Stairs, A. Brazier, P. Lazarus, R. Lynch, P. Scholz, K. Stovall, S. M. Ransom, S. Banaszak, C. M. Biwer, S. Cohen, L. P. Dartez, J. Flanigan, G. Lunsford, J. G. Martinez, A. Mata, M. Rohr, A. Walker, B. Allen, N. D. R. Bhat, S. Bogdanov, F. Camilo, S. Chatterjee, J. M. Cordes, F. Crawford, J. S. Deneva, G. Desvignes, R. D. Ferdman, P. C. C. Freire, J. W. T. Hessels, F. A. Jenet, D. L. Kaplan, V. M. Kaspi, B. Knispel, K. J. Lee, J. van Leeuwen, A. G. Lyne, M. A. McLaughlin, X. Siemens, L. G. Spitler, and A. Venkataraman, "Searching for Pulsars Using Image Pattern Recognition," The Astrophysical Journal, vol. 781, p. 117, Feb. 2014.

[138] X. Zhu and A. B. Goldberg, "Introduction to semi-supervised learning," Synthesis Lectures on Artificial Intelligence and Machine Learning, vol. 3, no. 1, pp. 1-130, 2009.

[139] Y. Zhu and D. Shasha, "Efficient elastic burst detection in data streams," in Proceedings of the Ninth ACM SIGKDD International Conference on Knowledge Discovery and Data Mining, ser. KDD '03. New York, NY, USA: ACM, 2003, pp. 336-345.

[140] A. Ziegler and I. R. König, "Mining data with random forests: current options for real-world applications," Wiley Interdisciplinary Reviews: Data Mining and Knowledge Discovery, vol. 4, no. 1, pp. 55-63, 2014. [Online]. Available: https://onlinelibrary.wiley.com/doi/abs/10.1002/widm.1114 